\title{
ITERATION TREES
}

\author{
D. A. MARTIN AND J. R. STEEL
}

\section{INTRODUCTION}

In this paper we shall develop some ideas which lead to a proof of the following

Theorem. Suppose there are $n$ Woodin cardinals, where $n<\omega$. Then there is a proper class model $M_{n}$ such that

$$
\begin{aligned}
M_{n} \models \text { ZFC } & + \text { "There are } n \text { Woodin cardinals" } \\
& + \text { " } \mathbb{R} \text { has a } \Delta_{n+2}^{1} \text { wellorder." }
\end{aligned}
$$

This theorem is best possible, in the sense that if there are $n$ Woodin cardinals and a measurable cardinal above them all, then $\mathbb{R}$ has no $\Delta_{n+2}^{1}$ wellorder [ShW], and in fact $\Pi_{n+1}^{1}$ determinacy holds [MS]. The theorem shows that the large cardinal hypothesis used to prove $\Pi_{n+1}^{1}$ determinacy in [MS] cannot be substantially improved.

Our theorem is an application of the theory of core models or canonical inner models for large cardinal hypotheses. Our work develops further some of the basic ideas of this theory. The theory itself has many other applications, particularly in the realm of relative consistency results. (As a quotable paradigm for a broad class of results, let us mention the following theorem of Solovay and Shelah: ZF + DC + "All sets of reals are Lebesgue measurable" is consistent if and only if $\mathrm{ZFC}+$ "There is an inaccessible cardinal" is consistent. In the "only if" direction, the model that one produces of ZFC + "There is an inaccessible cardinal" is a core model and, in fact, the smallest core model, Gödel's $L$.) We shall begin with a general description of core model theory, of some of its history, and of the advance we have made.

Many statements of mathematical interest cannot be decided in ZFC, the commonly accepted system of axioms for set theory. There are many widely varying and mutually incompatible ways of removing parts of this incompleteness. Among the multitude of corresponding models of ZFC, however, the class of core models stands out. The models in this class have a high degree of resemblance to one another, and there are powerful methods which produce a detailed account of their structure. On the other hand, the class seems rich enough to

Received by the editors March 14, 1991.

1991 Mathematics Subject Classification. Primary 03E15, 03E55; Secondary $03 E 60$.

The authors were partially supported by NSF Grants DMS89-02555 and DMS92-06946. 
contain models for all the known large cardinal hypotheses. Moreover, the core models are arranged in a natural wellordered hierarchy, a hierarchy that corresponds to the consistency-strength hierarchy on the large cardinal hypotheses they satisfy.

Every core model $M$ is an increasing wellordered union of approximating structures; we call $M$ and these approximations mice. In this terminology, a core model is a mouse which contains all the ordinals. Mice fall into a natural hierarchy. If $M$ and $N$ are mice, then $M \leq^{*} N$ iff there are mice $P$ and $Q$ and embeddings $j: M \rightarrow P$ and $k: N \rightarrow Q$ which are "produced by iteration" such that $P \subseteq Q$. (This slightly oversimplifies the correct definition.) The fundamental Comparison Lemma states that $\leq^{*}$ is connected; it is also reflexive, transitive, and wellfounded, and hence what is known as a prewellorder. If $M$ and $N$ are mice, then $M \leq^{*} N$ and $N \leq^{*} M$ just in case $M$ and $N$ "have a common iterate", that is, just in case there is a mouse $R$ and embeddings $j: M \rightarrow R$ and $k: N \rightarrow R$ "produced by iteration." The comparison process, whereby from mice $M$ and $N$ one produces by iteration $j: M \rightarrow P$ and $k: N \rightarrow Q$ such that either $P \subseteq Q$ or $Q \subseteq P$, is the main tool of core model theory.

One of the chief goals of core model theorists is to extend the theory to models further up in the mouse order $\leq^{*}$. This means, at a minimum, defining the models and showing that they exist (the construction problem) and making precise the phrase "produced by iteration" so that one can use iterations to prove a comparison lemma (the comparison problem). Underlying both of these problems is the iterability problem: one must show that the mice in the model one constructs behave well in the iterations one needs to compare them.

One important measure of progress is given by the large cardinal hypotheses satisfied in core models for which one can solve these problems. We believe that one day the theory will reach models for all the large cardinal hypotheses used by set theorists. This will mean that all of the many models of ZFC they have produced can be built by forcing from core models.

A second measure of progress is given by the logical complexity of the reals occurring in core models for which one has a theory. If $M$ is a mouse and $j: M \rightarrow P$ is produced by iteration, then $M$ and $P$ have the same reals. So if $M \leq^{*} N$, then $\mathbb{R} \cap M \subseteq \mathbb{R} \cap N$. On the other hand, if $M$ is a $\leq^{*}$-minimal mouse satisfying some sentence and if $M<^{*} N$, then $\mathbb{R} \cap M$ is countable in $N$. So as one goes up the mouse order, one finds more and more reals. The definability of $\leq^{*}$ can be used to show that these reals are definable. As one goes further up in $\leq^{*}$, the definitions become logically more complex.

A closely related measure is the logical complexity of the natural wellorder of the reals in a given core model. The reals occurring in mice are prewellordered by the places in the mouse order at which they appear: $x \leq^{* *} y$ iff for all mice $M \quad(y \in M \rightarrow x \in M)$. A slight refinement of $\leq^{* *}$ yields a wellorder of such reals, which we also call $\leq^{* *}$. If $M$ is a core model, then $M$ satisfies " $\leq^{* *}$ is a definable wellorder of $\mathbb{R}$ " and the complexity of the definition over $M$ of the restriction of $\leq^{* *}$ to reals in $M$ is closely related to the complexity of the comparison process as applied to mice below $M$ in the mouse order.

At this point we should warn the reader that there is no precise general def- 
inition of "core model", "mouse", or "mouse order", just as there is no such definition of "large cardinal hypothesis". One can only point to instances. The preceding paragraphs only describe the pattern of existing theory in these instances, although, as we said, we expect that one day this pattern will extend much further. We now turn to concrete results exhibiting the pattern.

We should note that one cannot construct any but the $\leq^{*}$-least core model without hypotheses going beyond ZFC. It is easiest to construct core models under large cardinal hypotheses, as we shall do in this paper, but for many applications it is important that one can develop the theory under other hypotheses. We shall not make explicit the hypotheses for the results described below; the existence of a supercompact cardinal is more than enough.

The $\leq^{*}$-least core model is $L$, the universe of constructible sets. Gödel introduced $L$ and established its basic properties in 1937 [G]. Later, around 1970 , Jensen produced a much more comprehensive and detailed theory of $L$ [J1]. (Jensen's "fine-structural" methods are invaluable throughout core model theory.) $L$ satisfies some weak large cardinal hypotheses, such as "There are inaccessible cardinals", but not much more. Every real in $L$ is $\Delta_{3}^{1}$, and $L$ satisfies " $\leq^{* *}$ is a $\Delta_{2}^{1}$ wellorder of $\mathbb{R}$ ".

The next core model studied was $L[U]$, the $\leq^{*}$ minimal core model satisfying "There is a measurable cardinal". Following a suggestion of Solovay, Silver [Si1, Si2] first defined $L[U]$ and proved for it the analogs of Gödel's results on $L$ (in 1966). Shortly afterward, Kunen [Ku] introduced more powerful methods and used them to re-do and extend Silver's work. In particular, he proved the first nontrivial comparison lemma. (The mice below $L$ are wellordered by inclusion, so no iteration is needed to compare them.)

$L[U]$ satisfies "There is a measurable cardinal" but no large cardinal hypotheses much stronger than this. The existence of a measurable cardinal implies that $\mathbb{R}$ has no $\Delta_{2}^{1}$ wellorder [So1], so there are reals in $L[U$ ] which are not in $L$. Nevertheless, every real in $L[U]$ is $\Delta_{3}^{1}$, and $L[U]$ satisfies " $\mathbb{R}$ has a $\Delta_{3}^{1}$ wellorder".

In [So2], Solovay extended Jensen's fine structure theory to $L[U]$. Building on this, Dodd and Jensen then (in 1976) produced a series of remarkable results which enable one to construct core models between $L$ and $L[U]$ under many widely varying hypotheses. (See [DJ1, DJ2, DJ3].)

The core models below $L[U]$ are too much a special case, however, to reveal the general form of a core model. In 1974 and 1978, Mitchell discovered what appear to be the basic features of this form [Mi1, Mi2]. He introduced models of the form $L[\vec{E}]$, where $\vec{E}$ is a coherent sequence of extenders. Roughly speaking, an extender is a system of ultrafilters which codes up an elementary embedding. If $E$ codes $j: M \rightarrow N$ and $F$ is an extender belonging to $N$, then we say $E$ is stronger than $F$. Speaking loosely again, a sequence of extenders is coherent just in case its members are listed in order of increasing strength and without leaving gaps. $L[U]$ is of the form $L[\vec{E}]$ for $\vec{E}$ a sequence of length 1 . One obtains core models further up in the mouse order by adding more extenders to the coherent sequence from which one constructs.

Mitchell developed a decent theory of models of the form $L[\vec{E}]$ satisfying 
large cardinal axioms well beyond the existence of a measurable cardinal. His goal had been a core model theory for supercompact cardinals, which seemed at the time to be the next natural large cardinal target after measurable cardinals, but he fell short of this. Baldwin and Dodd [B1, B2, D] extended Mitchell's work to somewhat larger cardinals, still short of supercompacts. About the same time (1980?), Mitchell produced a fine structure theory for the models of [Mi1] and used it to extend the Dodd-Jensen work on the construction problem to these models. (See [Mi4, Mi5].)

Once again, the reals in the Mitchell models are all $\Delta_{3}^{1}$, and these models satisfy " $\mathbb{R}$ has a $\Delta_{3}^{1}$ wellorder".

At the heart of the Kunen-Mitchell theory is a certain comparison process for mice of the form $L[\vec{E}]$. Given two models $M_{0}$ and $N_{0}$ of this form, one constructs by transfinite induction models $M_{\alpha}$ and $N_{\alpha}$ together with embeddings $i_{\beta \alpha}: M_{\beta} \rightarrow M_{\alpha}$ and $j_{\beta \alpha}: N_{\beta} \rightarrow N_{\alpha}$ for $\beta<\alpha$. If the coherent sequence of $M_{\alpha}$ is an initial segment of the coherent sequence of $N_{\alpha}$, or vice-versa, then the process stops and we have a successful comparison. Otherwise there is a least difference between the two coherent sequences. We let $i_{\alpha, \alpha+1}: M_{\alpha} \rightarrow M_{\alpha+1}$ and $j_{\alpha, \alpha+1}: N_{\alpha} \rightarrow N_{\alpha+1}$ be the embeddings coded by the extenders in $M_{\alpha}$ and $N_{\alpha}$ involved in this difference, and we continue to stage $\alpha+1$. At limit stages $\lambda$, we form $M_{\lambda}$ and $N_{\lambda}$ by taking direct limits. A system such as $\left\langle\left(M_{\beta}, i_{\beta, \alpha}\right) \mid \beta \leq \gamma \leq \alpha\right\rangle$ is called a linear iteration of $M_{0}$. A mouse $M$ is called linearly iterable if such an iteration beginning with $M=M_{0}$ will never lead to an illfounded $M_{\alpha}$. The mice in the Kunen-Mitchell models are all linearly iterable; this is the solution to the iterability problem in this region. (For mice in $L[U]$, the result is due to Gaifman; see [Ga]. Mitchell extended the result to cover the mice in $L[\vec{E}]$.) The fundamental Kunen-Mitchell comparison lemma states that if $M_{0}$ and $N_{0}$ are linearly iterable and "sufficiently small" (do not satisfy certain large cardinal axioms on the way to supercompactness) then the process of comparing them succeeds.

For countable mice, the property of being linearly iterable is $\Pi_{2}^{1}$ definable. This is the reason that the reals in core models $M$ containing only mice which are comparable via linear iteration are all $\Delta_{3}^{1}$. It is also the reason such $M$ satisfy " $\leq^{* *}$ is a $\Delta_{3}^{1}$ wellorder of $\mathbb{R}$ ". This meant one needed more than linear iteration to compare the mice in core models having non- $\Delta_{3}^{1}$ reals and made such models a natural next target for the theory.

In the period after Mitchell's [Mi2], core model theorists suffered from the illusion that the supercompact target was near at hand and the non- $\Delta_{3}^{1}$-real target much further off. Mitchell, and later Steel, experimented with nonlinear iterations but could not reach supercompacts using them. Then in 1984 Woodin, using a technical breakthrough of Foreman, Magidor, and Shelah [FMaSh], showed that if there is a supercompact cardinal, then $\mathbb{R}$ has no wellorder which is $\Delta_{n}^{1}$ for any $n<\omega$. Shelah and Woodin soon isolated what seemed to be the optimal hypothesis here [ShW]: they showed that the existence of $n$ Woodin cardinals with a measurable cardinal above them all implies that $\mathbb{R}$ has no $\Delta_{n+2}^{1}$ wellorder. (The rather involved definition of a Woodin cardinal would not il- 
luminate this discussion. See $\S 1$.) To core model theorists, these unexpected results meant that the comparison process must change in a significant way well before one got to supercompacts - in fact, at the level of Woodin cardinals (if not before). A core model theory for one Woodin cardinal was the natural next target.

In 1985, Steel [St1] used nonlinear iterations to prove a comparison lemma for mice at the bottom levels of a hierarchy leading to mice with one Woodin cardinal. This paper also extended Mitchell's coherent sequence framework in ways which have proved useful. Later in 1985, the authors isolated what seems to be a quite general type of nonlinear iteration and proved enough about it for the application [MS]. In the summer of 1986 we carried the analysis of this sort of iteration further and obtained thereby most of the results in this paper, including the theorem stated at the outset.

The model $M_{1}$ described in the theorem has a real in it which is not $\Delta_{3}^{1}$. Although $M_{1}$ satisfies " $\leq^{* *}$ is a $\Delta_{3}^{1}$ wellorder of $\mathbb{R}$ ", it is the $\leq^{*}$-largest core model for which this is true. In particular, $M_{2}$ satisfies " $\mathbb{R}$ has no $\Delta_{3}^{1}$ wellorder".

The basic way in which we change the Kunen-Mitchell comparison process is as follows. If at stage $\alpha$ in that process, the least difference between the mice $M_{\alpha}$ and $N_{\alpha}$ involves the extender $E$ from $M_{\alpha}$, then it can happen (once one gets beyond the models of [Mi2], [B1], and [D]) that $E$ actually codes an embedding $j: M_{\beta} \rightarrow P$ with domain $M_{\beta}$, for some $\beta<\alpha$, and not just an embedding with domain $M_{\alpha}$. The right thing to do in this case, it turns out, is to go back to the least $\stackrel{\alpha}{\beta}$ for which this is true, and let $M_{\alpha+1}$ be the corresponding model $P$. Doing this repeatedly, one produces a tree of models, with embeddings from the models earlier on a given branch to those later on the same branch. Such a system we call an iteration tree.

Much of this paper, $\S \S 1-4$, is devoted to the basic theory of iteration trees. We give the precise definition and prove some elementary facts about such trees in $\S 1$. Our main results concern the iterability problem; that is, the existence and uniqueness of iteration trees extending a given iteration tree. In $\S 2$ we deal with uniqueness and in $\S 4$ with existence. As the general existence proof of $\S 4$ is rather long and complicated, we have devoted $\S 3$ to special cases which illustrate some of its main ideas.

Although they suffice for our main theorem, the existence and uniqueness results of $\S \S 4$ and 2 are by no means conclusive. Much stronger statements seem likely true, and if so would be of great help in extending core model theory to still larger cardinals. We discuss these statements and some partial results concerning them in $\S 5$.

Core models and coherent sequences make no appearance in $\S \S 1-5$. They do appear in $\S 6$, where we prove the main theorem in the case $n=1$. The reader who would like a short trip through the core model theory in this paper can read $\S 1$, the statements of Theorems $2.3,2.4,4.3$, and 5.11 , and then $\S 6$. This should yield a reasonably good outline of the theory.

We shall finish the proof of the main theorem in a sequel to this paper. We shall also show, using the methods of [MS], that the model $M_{n}$ is $\Sigma_{n+1}^{1}$ correct. 
We should point out that the core model theory of $\S 6$ is severely limited by its use of an "external" comparison process. For example, we cannot decide even $\mathrm{GCH}$ in the model constructed in $\S 6$. By bringing fine structure techniques to bear, one can develop (along the same lines) a theory free of this limitation; this is done in [MiS]. Thus, the results of $\S 6$ are somewhat provisional in nature. Nevertheless, we believe $\S 6$ is a good introduction to the full theory one gets by bringing in fine structure.

Our use of an external comparison process also creates difficulties for the natural extension of the main theorem to models with infinitely many Woodin cardinals. By adding methods from Descriptive Set Theory to the mix, while retaining the external comparison process, Woodin has overcome some of these difficulties. In this way, for example, he has shown that if there are $\omega$ Woodin cardinals then there is a core model satisfying "there are $\omega$ Woodin cardinals" and " $\mathbb{R}$ has an $L(\mathbb{R})$-definable wellorder".

These techniques lead to inner models with appropriately definable wellorders of $\mathbb{R}$ for large cardinals somewhat stronger than measurable limits of Woodin cardinals. Somewhere between measurable limits and Woodin limits of Woodin cardinals, the weaknesses in our existence and uniqueness results on iteration tree extensions become a problem. (One can pin down more precisely the large cardinal level at which these weaknesses become a problem; see the remarks at the end of $\S 5$.) With proofs of the conjectures in $\S 5$, one could probably lift the theory to still larger cardinals.

The fine structure techniques of [MiS], which use an internal comparison process, also lead to an extension of the main theorem (and, in fact, a full core model theory) for infinitely many Woodin cardinals. This has been worked out in detail in [St2]. In this approach, the weaknesses in our results on iteration tree extension become a problem at the same large cardinal level as before.

Finally, Woodin has found purely descriptive set-theoretic proofs that, under appropriate determinacy hypotheses, there are inner models with various numbers of Woodin cardinals and appropriately definable wellorders of $\mathbb{R}$. For example, assuming $\Delta_{2}^{1}$ determinacy and letting $x \in \mathbb{R}$ code up winning strategies for all $\Delta_{2}^{1}$ games, $\mathrm{HOD}^{L[x]} \models$ "There is a Woodin cardinal and $\mathbb{R}$ has a $\Delta_{3}^{1}$ wellorder". These purely descriptive set-theoretic methods run into difficulties at the same large cardinal level that the other methods do, that is, somewhere below a Woodin limit of Woodin cardinals.

\section{Preliminaries; iteration trees}

A. Extenders. The notion of an extender is a simplification, due to Jensen, of a notion due to Mitchell.

Let $j: V \rightarrow M$ be elementary, where $V$ is the universe of all sets and $M$ is a transitive class. Let $\kappa=\operatorname{crit}(j)$ be the critical point of $j$, and $\kappa<\lambda \leq j(\kappa)$. For $a \subseteq \lambda$ finite and $X \subseteq[\kappa]^{|a|}$, let

$$
X \in E_{a} \Longleftrightarrow a \in j(X) .
$$

We call $E=\left\langle E_{a} \mid a \in[\lambda]^{<\omega}\right\rangle$ the $(\kappa, \lambda)$ extender derived from $j$.

If $b$ is a finite set of ordinals, then we sometimes use $b_{i}$ for the $i$ th element 
in the increasing enumeration of $b$. If $a \subseteq b \in[\lambda]^{n}$ and $a=\left\{b_{i_{1}} \cdots b_{i_{k}}\right\}$ where $i_{1}<\cdots<i_{k}$, then for $X \subseteq[\kappa]^{k}$ we let $X^{a b}=\left\{u \in[\kappa]^{n} \mid\left\{u_{i_{1}} \cdots u_{i_{k}}\right\} \in X\right\}$. Similarly, for $f:[\kappa]^{k} \rightarrow V$ we let $f^{a b}:[\kappa]^{n} \rightarrow V$ be given by $f^{a b}(u)=$ $f\left(\left\{u_{i_{1}} \cdots u_{i_{k}}\right\}\right)$. With this notation we can state the fundamental properties of the $(\kappa, \lambda)$ extender $\left\langle E_{a} \mid a \in[\lambda]^{<\omega}\right\rangle$ derived from $j$ :

1. Each $E_{a}$ is a $\kappa$-complete ultrafilter on $[\kappa]^{|a|}$. (We write "For $E_{a}$ a.e. $u, \varphi(u)$ " to mean $\{u \mid \varphi(u)\} \in E_{a}$. Thus, for example, for $E_{a}$ a.e. $u$, $u_{1}<\cdots<u_{|a|}$.

2. (Compatibility) If $a \subseteq b \in[\lambda]^{<\omega}$ and $X \in E_{a}$, then $X^{a b} \in E_{b}$.

3. (Normality) If $a \in[\lambda]^{<\omega}$ and $f(u)<u_{i}$ for $E_{a}$ a.e. $u$, then for some $\xi<a_{i}$ we have, letting $\xi$ be the $k$ th element of $a \cup\{\xi\}, f^{a, a \cup\{\xi\}}(u)=u_{k}$ for $E_{a \cup\{\xi\}}$ a.e. $u$.

4. ( $\omega$-Completeness) Let $a_{i} \in[\lambda]^{<\omega}$ and $X_{i} \in E_{a_{i}}$ for all $i<\omega$. Then there is an order-preserving $g: \bigcup_{i<\omega} a_{i} \rightarrow$ OR such that $g^{\prime \prime} a_{i} \in X_{i}$ for all $i$.

Given a system $E=\left\langle E_{a} \mid a \in[\lambda]^{<\omega}\right\rangle$ satisfying $1-4$ above, one can form $\operatorname{Ult}(V, E)$ in a natural way. We refer the reader to [MS] for the details. The elements of Ult $(V, E)$ are (the images under transitive collapse of ) equivalence classes of the form $[a, f]_{E}$, where $a \in[\lambda]^{<\omega}$ and $f:[\kappa]^{|a|} \rightarrow V$. (The equivalence relation is given by: $\langle a, f\rangle \sim\langle b, g\rangle$ iff $f^{a, a \cup b}(u)=g^{a, a \cup b}(u)$ for $E_{a \cup b}$ a.e. $u$.) We have a canonical elementary embedding $i_{E}: V \rightarrow \operatorname{Ult}(V, E)$ given by: $i_{E}(x)=\left[\{0\}, c_{x}\right]_{E}$, where $c_{x}(\alpha)=x$ for all $\alpha<\kappa$. It turns out that $E$ is the $(\kappa, \lambda)$ extender derived from $i_{E}$, so being a $(\kappa, \lambda)$ extender is equivalent to the conjunction of $1-4$.

If $E$ is the $(\kappa, \lambda)$ extender derived from $j$, where $j: V \rightarrow M$, then the diagram

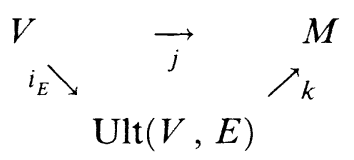

commutes, where $k\left([a, f]_{E}\right)=j(f)(a)$. Moreover, $k \uparrow \lambda=\mathrm{id}$. (id is the identity function.) It follows that if $M \models\left|V_{\eta}\right| \leq \lambda$, then $V_{\eta}^{M}=V_{\eta}^{\operatorname{Uit}(V, E)}$.

If $E$ is a $(\kappa, \lambda)$ extender, then we call $\kappa$ the critical point of $E$ and $\lambda$ the length of $E$ and write $\kappa=\operatorname{crit}(E)$ and $\lambda=\operatorname{lh}(E)$. The strength of $E$, or strength $(E)$, is the largest $\eta$ such that $V_{\eta} \subseteq \operatorname{Ult}(V, E)$. Notice that $\kappa+1 \leq \operatorname{strength}(E) \leq \lambda$; the latter inequality holds because $E$ can be coded by a member of $V_{\lambda+1}$, and $E \notin \operatorname{Ult}(V, E)$. (If $E \in \operatorname{Ult}(V, E)$, then $i_{E}\left(\kappa^{+}\right)$ has cardinality $i_{E}(\kappa)$ in $\operatorname{Ult}(V, E)$, a contradiction.)

If $M$ is a model of a reasonable fragment of $\mathrm{ZFC}$ and $M \vDash$ " $E$ is a $(\kappa, \lambda)$ extender", then one can form $\operatorname{Ult}(M, E)$ simply by relativising the construction of $\operatorname{Ult}(V, E)$ to $M$. In fact, if $V_{\kappa+1}^{M}=V_{\kappa+1}^{N}$, then one can also form $\operatorname{Ult}(N, E)$; its elements are equivalence classes $[a, f]_{E}^{N}$, where $a \in[\lambda]^{<\omega}$ and $f:[\kappa]^{|a|} \rightarrow N$ and $f \in N . E$ measures enough sets that the 
construction makes sense (although we may have $\lambda>\mathrm{OR}^{N}$ ). See [MS] for details. Notice that although $\operatorname{Ult}(M, E)$ must be wellfounded, provided $M$ is, $\operatorname{Ult}(N, E)$ need not be wellfounded. (There are counterexamples.) By $i_{E}^{N}$ we mean the canonical embedding from $N$ into $\operatorname{Ult}(N, E)$.

A cardinal $\delta$ is Woodin just in case whenever $f: \delta \rightarrow \delta$ there is a $\kappa<\delta$ such that $f^{\prime \prime} \kappa \subseteq \kappa$ and an elementary embedding $j: V \rightarrow M$ with critical point $\kappa$ such that $V_{j(f)(\kappa)} \subseteq M$. It is not hard (but not trivial) to show that if $\delta$ is Woodin, then it is Woodin via extenders in $V_{\delta}$. We shall take this as our official definition.

Definition 1.0. A cardinal $\delta$ is Woodin iff $\forall f: \delta \rightarrow \delta \quad \exists \kappa<\delta\left(f^{\prime \prime} \kappa \subseteq \kappa \wedge \exists E \in\right.$ $V_{\delta}\left(E\right.$ is an extender $\left.\left.\wedge \operatorname{crit}(E)=\kappa \wedge \operatorname{strength}(E) \geq i_{E}(f)(\kappa)\right)\right)$.

It is clear that if $\delta$ is Woodin, then $\delta$ is strongly inaccessible and, in fact, Mahlo. On the other hand, the least Woodin cardinal is not weakly compact. Let us say that $\kappa$ is $\lambda$-strong iff $\exists j: V \rightarrow M\left(\operatorname{crit}(j)=\kappa \wedge V_{\lambda} \subseteq M\right)$. It is easy to show that if $\delta$ is Woodin, then $\delta$ is a limit of cardinals $\kappa$ such that $\forall \lambda<\delta$ ( $\kappa$ is $\lambda$-strong).

B. Premice. We shall need to work with models of fragments of ZFC. We shall arrange things so that, nevertheless, all ultrapowers we form satisfy the full Los theorem.

Definition 1.1. Let $M$ be a transitive set or class and $\delta \in \mathrm{OR}^{M}$. The structure $\mathscr{M}=(M, \in, \delta)$ is a premouse iff $\mathscr{M}$ satisfies the following axioms: nullset, pairing, union, infinity, powerset, choice, the full separation schema, the $\Sigma_{1}(\mathscr{P})$ collection schema, and the full collection schema "for domains $\subseteq V_{\delta}$ " (that is, each instance of $\left.\forall t\left[\forall x \in V_{\delta} \exists u \varphi(x, u, t) \Rightarrow \exists v \forall x \in V_{\delta} \exists u \in v \varphi(x, u, t)\right]\right)$.

Here $\Sigma_{1}(\mathscr{P})$ formulas are $\Sigma_{1}$ formulas with an additional unary function symbol always interpreted by the power set function. Thus a premouse is power admissible and, in particular, satisfies " $V_{\alpha}$ exists, for all ordinals $\alpha$ ".

If $\mathscr{M}=(M, \in, \delta)$ is a premouse, then we write $\delta=\delta^{\mathscr{M}}$. We may identify $\mathscr{M}$ and $M$ in contexts where $\delta$ is not important.

We shall state our general extendibility results on iteration trees in terms of premice. When we apply them to the comparison of inner models, $\delta^{\mathscr{M}}$ will be the length of a coherent sequence in $M$. Notice here that if $\mathscr{M}$ and $\mathscr{N}$ are premice, $\mathscr{M} \vDash$ “ $E$ is a $(\kappa, \lambda)$ extender", $V_{\kappa+1}^{M}=V_{\kappa+1}^{N}$, and $\kappa<\delta^{\mathscr{N}}$, then the full Loś theorem holds for the ultrapower $\operatorname{Ult}(\mathscr{N}, E)$, so that $i_{E}^{\mathscr{N}}$ is fully elementary.

Definition 1.2. We say premice $\mathscr{M}$ and $\mathscr{N}$ agree through $\eta$ iff $V_{\eta}^{\mathscr{M}}=V_{\eta}^{\mathscr{N}}$.

\section{Iteration trees.}

Definition 1.3. Let $\mathrm{T}$ be a strict partial order on an ordinal $\gamma$. We call $\mathrm{T}$ a tree order of $\gamma$ iff

(i) $\forall \beta<\gamma$ ( $\mathrm{T}$ wellorders $\{\alpha \mid \alpha \mathrm{T} \beta\})$,

(ii) $\forall \alpha \beta(\alpha \mathrm{T} \beta \Rightarrow \alpha<\beta)$,

(iii) $\forall \alpha(0<\alpha \Rightarrow 0 \mathrm{~T} \alpha)$, 
(iv) $\forall \alpha(\alpha$ is a successor ordinal $\Leftrightarrow \alpha$ is a T-successor), and

(v) $\forall \lambda<\gamma(\lambda$ is a limit ordinal $\Rightarrow\{\alpha \mid \alpha \mathrm{T} \lambda\}$ is $\in$-cofinal in $\lambda)$.

We shall use the usual interval notation when speaking of tree orders: $[\alpha, \beta]_{\mathrm{T}}$ $=\{\gamma \mid \gamma=\alpha$ or $(\alpha \mathrm{T} \gamma$ and $\gamma \mathrm{T} \beta)$ or $\gamma=\beta\},[\alpha, \beta)_{\mathrm{T}}=\{\gamma \mid \gamma=\alpha$ or $(\alpha \mathrm{T} \gamma$ and $\gamma \mathrm{T} \beta)\}$, etc. By T-pred $(\alpha)$ we mean the unique $\beta \mathrm{T} \alpha$ such that $(\beta, \alpha)_{\mathrm{T}}=\varnothing$, if any such $\beta$ exists.

Definition 1.4. Let $\mathscr{M}$ be a premouse. An iteration tree on $\mathscr{M}$ of length $\theta$ is a pair $\mathscr{T}=\left\langle\mathrm{T},\left\langle E_{\alpha} \mid \alpha+1<\theta\right\rangle\right\rangle$ such that $\mathrm{T}$ is a tree order on $\theta$ and there are premice $\mathscr{M}_{\alpha}, \alpha<\theta$, and embeddings $i_{\alpha \beta}: \mathscr{M}_{\alpha} \rightarrow \mathscr{M}_{\beta}$ defined when $\alpha \mathrm{T} \beta$, satisfying the following conditions:

(a) $\mathscr{M}_{0}=\mathscr{M}$.

(b) If $\alpha+1<\theta$, then $E_{\alpha} \in V_{\delta}^{M_{\alpha}}$, where $\delta=\delta^{M_{\alpha}}$; moreover, $\mathscr{M}_{\alpha} \vDash$ “ $E_{\alpha}$ is an extender".

(c) Let $\alpha+1<\theta$ and $\beta=\mathrm{T}$-pred $(\alpha+1)$. Then $\mathscr{M}_{\beta}$ agrees with $\mathscr{M}_{\alpha}$ through $\operatorname{crit}\left(E_{\alpha}\right)+1 ;$ moreover,

$$
\mathscr{M}_{\alpha+1}=\operatorname{Ult}\left(\mathscr{M}_{\beta}, E_{\alpha}\right)
$$

and $i_{\beta, \alpha+1}$ is the canonical embedding from $\mathscr{M}_{\beta}$ into $\operatorname{Ult}\left(\mathscr{M}_{\beta}, E_{\alpha}\right)$, and for $\eta \mathrm{T} \beta, i_{\eta, \alpha+1}=i_{\beta, \alpha+1} \circ i_{\eta, \beta}$.

(d) Let $\lambda<\theta$ be a limit ordinal. Then $\mathscr{M}_{\lambda}=$ the direct limit of the $\mathscr{M}_{\alpha}$, $\alpha \mathrm{T} \lambda$, under the $i_{\alpha \beta}$ 's, and for $\alpha \mathrm{T} \lambda, i_{\alpha \lambda}$ is the canonical embedding.

An iteration tree $\mathscr{T}$ and its base model $\mathscr{M}_{0}$ uniquely determine the subsequent models $\mathscr{M}_{\alpha}$ and embeddings $i_{\alpha \beta}$. We sometimes write $E_{\alpha}^{\mathscr{T}}, \mathscr{M}_{\alpha}^{\mathscr{T}}$, and $i_{\alpha \beta}^{\mathscr{T}}$; respectively, for the extenders, models, and embeddings associated to $\mathscr{T}$ and, through context, $\mathscr{M}_{0}$.

The agreement between the models of $\mathscr{T}$ is related to the strength of the extenders used in $\mathscr{T}$.

Lemma 1.5. Let $\mathscr{M}$ and $\mathcal{N}$ be premice which agree through $\kappa+1$, where $\kappa=\operatorname{crit}(E)$ for some $E$ and such that $\mathscr{M} \vDash$ " $E$ is an extender". Suppose $\operatorname{Ult}(\mathcal{N}, E)$ is wellfounded. Then:

(a) $i_{E}^{\mathscr{N}}(\kappa)=i_{E}^{\mathscr{M}}(\kappa)$, and $\operatorname{Ult}(\mathscr{N}, E)$ agrees with $\operatorname{Ult}(\mathscr{M}, E)$ through $i_{E}^{\mathscr{N}}(\kappa)+1$

(b) $\operatorname{Ult}(\mathcal{N}, E)$ agrees with $\mathscr{M}$ through $\operatorname{strength}^{\mathscr{M}}(E)$;

(c) $V_{\rho+1}^{\mathrm{Ult}(\mathcal{N}, E)} \subsetneq V_{\rho+1}^{\mathscr{M}}$, where $\rho=\operatorname{strength}^{\mathscr{K}}(E)$.

Proof. If $f:[\kappa]^{n} \rightarrow V_{\kappa+1}$, then $f \in \mathscr{M}$ iff $f \in \mathscr{N}$. Thus $i_{E}^{\mathscr{N}}\left(V_{\kappa+1}^{\mathscr{K}}\right)=$ $i_{E}^{\mathscr{N}}\left(V_{\kappa+1}^{\mathscr{N}}\right)$, as the two are computed using the same functions. This proves (a). We get (b) and (c) at once from (a) and the fact that strength ${ }^{\mathscr{K}}(E) \leq i_{E}^{\mathscr{K}}(\kappa)$.

Definition 1.6. Let $\mathscr{T}$ be an iteration tree on $\mathscr{M}_{0}$, and $\alpha<\beta<\operatorname{lh}(\mathscr{T})$. Then

$$
\rho^{\mathscr{g}}(\alpha, \beta)=\inf \left\{\operatorname{strength}^{\mathscr{M}_{\gamma}^{\mathscr{G}}}\left(E_{\gamma}^{\mathscr{G}}\right) \mid \alpha \leq \gamma<\beta\right\}
$$


Lemma 1.7. Let $\mathscr{T}$ be an iteration tree on $\mathscr{M}_{0}$ and $\alpha<\beta<\operatorname{lh}(\mathscr{T})$. Then

(a) $\mathscr{M}_{\alpha}^{\mathscr{T}}$ agrees with $\mathscr{M}_{\beta}^{\mathscr{T}}$ through $\rho^{\mathscr{T}}(\alpha, \beta)$;

(b) $V_{\rho+1}^{\mathscr{M}_{\beta}^{\mathscr{I}}} \subsetneq V_{\rho+1}^{\mathscr{M}_{\alpha}^{\mathscr{T}}}$, where $\rho=\rho^{\mathscr{T}}(\alpha, \beta)$.

Proof. We fix $\alpha$ and prove (a) and (b) by induction on $\beta$. First, let $\beta=\delta+1$ and $\kappa=\operatorname{crit}\left(E_{\delta}^{\mathscr{T}}\right), \nu=\operatorname{strength}^{\mathscr{M}_{\delta}^{\mathscr{G}}}\left(E_{\delta}^{\mathscr{T}}\right)$, and $\gamma=\mathrm{T}$-pred $(\beta)$. By Lemma 1.5, $\mathscr{M}_{\beta}^{\mathscr{I}}=\operatorname{Ult}\left(\mathscr{M}_{\gamma}^{\mathscr{T}}, E_{\delta}^{\mathscr{T}}\right)$ agrees with $\mathscr{M}_{\delta}^{\mathscr{T}}$ through $\nu . \mathscr{M}_{\delta}^{\mathscr{T}}$ agrees with $\mathscr{M}_{\alpha}^{\mathscr{T}}$ through $\rho^{\mathscr{T}}(\alpha, \delta)$ if $\alpha<\delta$; otherwise, $\mathscr{M}_{\delta}^{\mathscr{T}}=\mathscr{M}_{\alpha}^{\mathscr{T}}$. As $\rho^{\mathscr{T}}(\alpha, \beta)=$ $\inf \left\{\nu, \rho^{\mathscr{T}}(\alpha, \delta)\right\}$, we have (a).

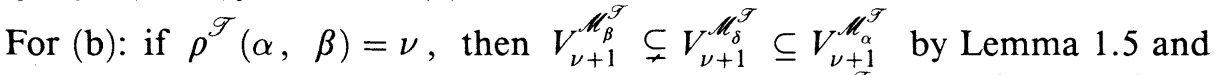
induction. If $\rho^{\mathscr{T}}(\alpha, \beta)=\rho^{\mathscr{T}}(\alpha, \delta)=\rho$, then $V_{\rho+1}^{\mathscr{M}_{\beta}^{\mathscr{T}}} \subseteq V_{\rho+1}^{\mathscr{M}_{\delta}^{\mathcal{G}}} \subsetneq V_{\rho+1}^{\mathscr{M}_{\alpha}^{\mathscr{T}}}$ by Lemma 1.5 and induction. (We skip the case $\alpha=\delta$.)

Next, let $\beta$ be a limit ordinal. Now $\operatorname{lh}\left(E_{\gamma}^{\mathscr{I}}\right) \geq \rho^{\mathscr{T}}(\alpha, \beta)$ for $\alpha \leq \gamma<\beta$, and since $\mathscr{M}_{\beta}^{\mathscr{T}}$ is wellfounded, there must be $\eta<\beta$ such that $\operatorname{crit}\left(E_{\gamma}^{\mathscr{T}}\right)>$ $\rho^{\mathscr{T}}(\alpha, \beta)$ whenever $\gamma \geq \eta$ and $(\gamma+1) \mathrm{T} \beta$. (Otherwise, there are arbitrarily large $(\gamma+1) \mathrm{T} \beta$ such that, letting $\theta=\mathrm{T}-\operatorname{pred}(\gamma+1), i_{\theta, \gamma+1}^{\mathscr{T}}\left(\rho^{\mathscr{T}}(\alpha, \beta)\right)>$ $\rho^{\mathscr{T}}(\alpha, \beta)$. This implies that $\left\{i_{\theta \beta}^{\mathscr{T}}\left(\rho^{\mathscr{T}}(\alpha, \beta)\right) \mid \theta \mathrm{T} \beta\right\}$ is a subset of $\mathscr{M}_{\beta}^{\mathscr{T}}$ with no $\in$-minimal element.) We may assume $\eta \mathrm{T} \beta, \eta>\alpha$, and $\rho^{\mathscr{T}}(\alpha, \beta)=$ $\rho^{\mathscr{T}}(\alpha, \eta)$. But then $\operatorname{crit}\left(i_{\eta \beta}^{\mathscr{T}}\right)>\rho^{\mathscr{T}}(\alpha, \eta)$, so $\mathscr{M}_{\beta}^{\mathscr{T}}$ agrees with $\mathscr{M}_{\eta}^{\mathscr{T}}$ through $\rho^{\mathscr{T}}(\alpha, \eta)+1=\rho^{\mathscr{T}}(\alpha, \beta)+1$, and we get $(\mathrm{a})$ and $(\mathrm{b})$ from the induction hypothesis at $\eta$.

Unfortunately, we must restrict the general notion of an iteration tree a bit in order to prove our results on the existence of extensions. This is almost certainly due to weaknesses in the proofs. One sort of restriction that suffices is given in the following definition.

Definition 1.8. Let $\mathscr{T}$ be an iteration tree on some $\mathscr{M}$, and let $n<\omega$. We say $\mathscr{T}$ is a plus $n$ tree iff whenever $\beta+1<\operatorname{lh}(\mathscr{T})$,

$$
\sup \left\{\operatorname{crit}\left(E_{\gamma}^{\mathscr{T}}\right) \mid \beta+1 \leq \gamma \wedge \mathrm{T}-\operatorname{pred}(\gamma+1) \leq \beta\right\}+n \leq \rho^{\mathscr{T}}(\beta, \beta+1) .
$$

Here and in the rest of this paper, $\sup (\varnothing)=0$.

Of course, $\rho^{\mathscr{T}}(\beta, \beta+1)$ is just the strength of $E_{\beta}^{\mathscr{T}}$ in $\mathscr{M}_{\beta}^{\mathscr{T}}$. We shall need to restrict, at most, to plus 2 trees. The notion of iteration tree defined in Definition 1.4 is somewhat more general than that defined in [MS] or in the notes on which this paper is based. The following definition captures the earlier, more restrictive notion.

Definition 1.9. An iteration tree $\mathscr{T}$ is normal iff there are ordinals $\rho_{\alpha}$, for $\alpha+1<\operatorname{lh}(\mathscr{T})$, such that for all $\alpha+1, \beta+1<\operatorname{lh}(\mathscr{T})$

(1) $\rho_{\alpha}+2 \leq \rho^{\mathscr{T}}(\alpha, \alpha+1)$,

(2) $\alpha<\beta \Rightarrow \rho_{\alpha}<\rho_{\beta}$, and 
(3) $T-\operatorname{pred}(\alpha+1)=$ least $\gamma \leq \alpha$ such that $\operatorname{crit}\left(E_{\alpha}^{\mathscr{T}}\right) \leq \rho_{\gamma}$.

If $\mathscr{T}$ is normal, as witnessed by $\left\langle\rho_{\alpha} \mid \alpha+1<\operatorname{lh}(\mathscr{T})\right\rangle$, then $\rho_{\alpha}+2 \leq$ $\rho^{\mathscr{T}}(\alpha, \beta)$ for all $\alpha<\beta$ and $\operatorname{crit}\left(E_{\beta}^{\mathscr{T}}\right) \leq \rho_{\alpha}$ whenever $T-\operatorname{pred}(\beta+1) \leq \alpha$. So every normal tree is a plus 2 tree.

The iteration trees we need in this paper are all normal. These trees arise from the comparison process of $\S 6$, and in this case $\rho_{\alpha}$ measures the agreement between $\mathscr{M}_{\alpha}^{\mathscr{T}}$ and some model with which we are comparing it. On the other hand, nonnormal trees have some uses (e.g., in the Dodd-Jensen lemma of $[\mathrm{MiS}])$, and the extra generality causes only notational awkwardness.

\section{UNIQUENESS OF WELLFOUNDED BRANCHES}

A branch of a tree order $\mathrm{T}$ is a set $b$ linearly ordered by $\mathrm{T}$, having no last element, and such that $\alpha \in b \rightarrow[0, \alpha]_{\mathrm{T}} \subseteq b$. A branch $b$ of $\mathrm{T}$ is cofinal iff $\forall \alpha \in \operatorname{field}(\mathrm{T}) \exists \beta \in b(\alpha<\beta)$. A branch $b$ of $\mathrm{T}$ is maximal iff $b \neq[0, \lambda)_{\mathrm{T}}$ for all $\lambda$. Cofinal branches must be maximal, but a maximal branch may not be cofinal. Notice that if $\lambda$ is a limit ordinal in the field of $T$, then $[0, \lambda)_{\mathrm{T}}$ is the unique nonmaximal branch of $T$ having supremum $\lambda$. Also, every branch $b$ of $\mathrm{T}$ is closed below its sup; that is, if $\alpha<\sup b$, then $\sup (b \cap \alpha) \in b$. These facts follow from clauses (iv) and (v) of Definition 1.3.

If $\mathscr{T}$ is an iteration tree, then a branch of $\mathscr{T}$ is just a branch of the associated tree order.

Definition 2.1. Let $b$ be a branch of the iteration tree $\mathscr{T}$. Then

$$
\mathscr{M}_{b}^{\mathscr{T}}=\text { the direct limit of the } \mathscr{M}_{\alpha}^{\mathscr{T}}, \alpha \in b, \text { under the } i_{\alpha, \beta}^{\mathscr{I}} \text {. }
$$

For $\alpha \in b, i_{\alpha, b}^{\mathscr{T}}$ is the canonical embedding from $\mathscr{M}_{\alpha}^{\mathscr{T}}$ into $\mathscr{M}_{b}^{\mathscr{T}}$. We say $b$ is wellfounded just in case $\mathscr{M}_{b}^{\mathscr{T}}$ is wellfounded.

As always, we shall identify $\operatorname{wfp}\left(\mathscr{M}_{b}^{\mathscr{T}}\right)$, the wellfounded part of $\mathscr{M}_{b}^{\mathscr{T}}$, with the transitive set to which it is isomorphic.

The Unique Branches Hypothesis, or UBH, asserts that every iteration tree on $V$ has at most one cofinal wellfounded branch. The truth of the UBH is the most important open problem concerning iteration trees, and we shall discuss some partial results that concern it in $\S 5$. Our next theorem and corollary can be viewed as proof of a weakening of the UBH, but they go beyond this in an important way, in that they concern trees merely on-not necessarily in-the base model.

If $\mathscr{T}$ is an iteration tree of limit length $\lambda$, then we set, for $\alpha<\lambda$,

$$
\rho^{\mathscr{T}}(\alpha, \lambda)=\inf \left\{\operatorname{strength}^{\mathscr{M}_{\gamma}^{\mathscr{T}}}\left(E_{\gamma}^{\mathscr{T}}\right) \mid \alpha \leq \gamma<\lambda\right\} .
$$

If $b$ is a cofinal branch of $\mathscr{T}$ and $\rho^{\mathscr{T}}(\alpha, \lambda) \in \operatorname{wfp}\left(\mathscr{M}_{b}^{\mathscr{T}}\right)$, then $\mathscr{M}_{b}^{\mathscr{T}}$ agrees with $\mathscr{M}_{\alpha}^{\mathscr{T}}$ through $\rho^{\mathscr{T}}(\alpha, \lambda)$. (If not, then $\operatorname{crit}\left(i_{\theta b}^{\mathscr{T}}\right)<\rho^{\mathscr{F}}(\alpha, \lambda)$ for all $\theta \in b$. Thus there are arbitrarily large $\gamma+1 \in b$ such that, letting $\theta=\mathrm{T}$-pred $(\gamma+1)$, we have $\operatorname{crit}\left(i_{\theta, \gamma+1}^{\mathscr{T}}\right)<\rho^{\mathscr{T}}(\alpha, \lambda)$ and $i_{\theta, \gamma+1}^{\mathscr{T}}\left(\operatorname{crit}\left(i_{\theta, \gamma+1}^{\mathscr{T}}\right)\right) \geq \rho^{\mathscr{T}}(\alpha, \lambda)$. This 
implies $\mathscr{M}_{b}^{\mathscr{T}}$ is illfounded, and in fact $\sup \left\{\operatorname{crit}\left(i_{\theta b}^{\mathscr{T}}\right) \mid \theta \in b\right\}=\mathrm{OR} \cap \mathrm{wfp}\left(\mathscr{M}_{b}^{\mathscr{T}}\right)$. Thus (OR $\left.\cap \operatorname{wfp}\left(\mathscr{M}_{b}^{\mathscr{T}}\right)\right) \leq \rho^{\mathscr{T}}(\alpha, \lambda)$, contrary to hypothesis.) Notice that if $\alpha<\beta<\lambda$, then $\rho^{\mathscr{T}}(\alpha, \lambda) \leq \rho^{\mathscr{T}}(\beta, \lambda)$. Thus if $b$ and $c$ are cofinal branches of $\mathscr{T}$ such that $\sup \left\{\rho^{\mathscr{T}}(\alpha, \lambda) \mid \alpha<\lambda\right\}=\theta$ belongs to $\operatorname{wfp}\left(\mathscr{M}_{b}^{\mathscr{T}}\right) \cap \operatorname{wfp}\left(\mathscr{M}_{c}^{\mathscr{T}}\right)$, then $\mathscr{M}_{b}^{\mathscr{I}}$ agrees with $\mathscr{M}_{c}^{\mathscr{T}}$ through $\theta$.

Theorem 2.2. Let $\mathscr{T}$ be an iteration tree of limit length $\lambda$, and let $b$ and $c$ be distinct cofinal branches of $\mathscr{T}$. Let $\theta=\sup \left\{\rho^{\mathscr{T}}(\alpha, \lambda) \mid \alpha<\lambda\right\}$, and suppose $\theta \in \operatorname{wfp}\left(\mathscr{M}_{b}^{\mathscr{T}}\right) \cap \operatorname{wfp}\left(\mathscr{M}_{c}^{\mathscr{T}}\right)$. Let $f: \theta \rightarrow \theta$ and $f \in \mathscr{M}_{b}^{\mathscr{T}} \cap \mathscr{M}_{c}^{\mathscr{T}}$. Then $\mathscr{M}_{b}^{\mathscr{T}} \models " \theta$ is Woodin with respect to $f$ "; that is,

$\mathscr{M}_{b}^{\mathscr{T}} \vDash \exists \kappa<\theta\left[f^{\prime \prime} \kappa \subseteq \kappa \wedge \exists E\right.$

( $E$ is an extender $\left.\left.\wedge \operatorname{crit}(E)=\kappa \wedge \operatorname{strength}(E)>i_{E}(f)(\kappa)\right)\right]$.

Proof. We show first that $\theta=\sup \left\{\operatorname{crit}\left(i_{\gamma b}\right) \mid \gamma \in b\right\}=\sup \left\{\operatorname{crit}\left(i_{\gamma c}\right) \mid \gamma \in c\right\}$. (Here and for the rest of the proof we omit the superscript “ $\mathscr{T}$ ".) For let $\gamma \in b$; we claim $\operatorname{crit}\left(i_{\gamma b}\right)<\theta$. To see this, choose $\xi$ such that $\gamma<\xi<\lambda$ and strength ${ }^{\mathscr{M}_{\xi}}\left(E_{\xi}\right) \leq \theta$. If $\xi+1 \in b$, then $E_{\xi}$ is one of the extenders used after $\gamma$ on $b$, so $\operatorname{crit}\left(i_{\gamma b}\right) \leq \operatorname{crit}\left(E_{\xi}\right)<\operatorname{strength}^{\mathscr{M}_{\xi}}\left(E_{\xi}\right) \leq \theta$, and we are done. If $\xi+1 \notin b$, let $\eta+1>\xi+1$ be least such that $\eta+1 \in b$. Then

$$
\mathrm{T}-\operatorname{pred}(\eta+1) \leq \xi<\eta,
$$

so $\rho(\mathrm{T}-\operatorname{pred}(\eta+1), \eta) \leq \operatorname{strength}^{\mathscr{M}_{\xi}}\left(E_{\xi}\right)$ and, hence, $\operatorname{crit}\left(E_{\eta}\right)<\operatorname{strength}^{\mathscr{M}_{\xi}}\left(E_{\xi}\right)$. As $E_{\eta}$ is used after $\gamma$ on $b$, again we are done. Thus $\gamma \in b \Rightarrow \operatorname{crit}\left(i_{\gamma b}\right)<\theta$. On the other hand, let $\beta<\theta$. Then $\beta<\rho(\delta, \lambda)$ for some $\delta<\lambda$, so $\beta<$ strength $^{\mathscr{M}_{\xi}}\left(E_{\xi}\right)$ for all $\xi \geq \delta$. So all extenders used on $b$ after $\delta$ map their critical points above $\beta$. As $\theta \in \operatorname{wfp}\left(\mathscr{M}_{b}\right)$, all but finitely many such extenders have critical point above $\beta$. So for $\gamma \in b$ sufficiently large, $\operatorname{crit}\left(i_{\gamma b}\right)>\beta$. Thus $\theta=\sup \left\{\operatorname{crit}\left(i_{\gamma b}\right) \mid \gamma \in b\right\} . \theta=\sup \left\{\operatorname{crit}\left(i_{\gamma c}\right) \mid \gamma \in c\right\}$ is proved symmetrically.

We now define ordinals $\alpha_{n} \in b, \beta_{n} \in c$ converging to $\lambda$ and ordinals $\kappa_{n}, \nu_{n}$ converging to $\theta$. Let

$$
\begin{aligned}
& \kappa_{0}=\text { least } \kappa \text { such that } \kappa=\operatorname{crit}\left(E_{\alpha}\right) \text { for some } \alpha \text { with } \alpha+1 \in b \backslash c, \\
& \alpha_{0}=\text { largest } \alpha \text { such that } \kappa_{0}=\operatorname{crit}\left(E_{\alpha}\right) \text { and } \alpha+1 \in b .
\end{aligned}
$$

Now given $\kappa_{n}$ and $\alpha_{n}$, set

$\nu_{n}=$ least $\nu$ such that $\nu=\operatorname{crit}\left(E_{\beta}\right)$ for some $\beta$ with $\beta+1 \in c \backslash\left(\alpha_{n}+1\right)$,

$\beta_{n}=$ largest $\beta$ such that $\nu_{n}=\operatorname{crit}\left(E_{\beta}\right)$ and $\beta+1 \in c$,

$\kappa_{n+1}=$ least $\kappa$ such that $\kappa=\operatorname{crit}\left(E_{\alpha}\right)$ for some $\alpha$ with $\alpha+1 \in b \backslash\left(\beta_{n}+1\right)$,

$\alpha_{n+1}=$ largest $\alpha$ such that $\kappa_{n+1}=\operatorname{crit}\left(E_{\alpha}\right)$ and $\alpha+1 \in b$.

Clearly, each of the sequences $\left\langle\alpha_{n} \mid n<\omega\right\rangle,\left\langle\beta_{n} \mid n<\omega\right\rangle,\left\langle\kappa_{n} \mid n<\omega\right\rangle$, and $\left\langle\nu_{n} \mid n<\omega\right\rangle$ is strictly increasing. As $\alpha_{n}<\beta_{n}<\alpha_{n+1}, \sup \left\{\alpha_{n}+1 \mid n<\omega\right\}=$ $\sup \left\{\beta_{n}+1 \mid n<\omega\right\}$. If $\sup \left\{\alpha_{n}+1 \mid n<\omega\right\}<\lambda$, then as $b$ and $c$ are closed 
in $\lambda, \sup \left\{\alpha_{n}+1 \mid n<\omega\right\} \in b \cap c$. This contradicts the choice of $\alpha_{0}$. Thus $\sup \left\{\alpha_{n}+1 \mid n<\omega\right\}=\sup \left\{\alpha_{n} \mid n<\omega\right\}=\sup \left\{\beta_{n} \mid n<\omega\right\}=\lambda$. It follows from our earlier observations that $\sup \left\{\kappa_{n} \mid n<\omega\right\}=\sup \left\{\nu_{n} \mid n<\omega\right\}=\theta$.

We claim the $E_{\alpha_{n}}$ 's and $E_{\beta_{n}}$ 's "overlap" in the following way:

$$
\begin{gathered}
\nu_{n}=\operatorname{crit}\left(E_{\beta_{n}}\right)<\operatorname{strength}^{\mathscr{M}_{\alpha_{n}}}\left(E_{\alpha_{n}}\right), \\
\kappa_{n+1}=\operatorname{crit}\left(E_{\alpha_{n+1}}\right)<\operatorname{strength}^{\mathscr{M}_{\beta_{n}}}\left(E_{\beta_{n}}\right) .
\end{gathered}
$$

We show the first inequality, the proof of the second being similar. Let $\gamma$ be least such that $\gamma+1>\alpha_{n}+1$ and $\gamma+1 \in c$. Let $\delta=\mathrm{T}$-pred $(\gamma+1)$ so that $\delta<$ $\alpha_{n}+1$. As $\delta \leq \alpha_{n}<\gamma, \rho(\delta, \gamma) \leq \operatorname{strength}^{\mathscr{M}_{\alpha_{n}}}\left(E_{\alpha_{n}}\right)$. But $\operatorname{crit}\left(E_{\gamma}\right)<\rho(\delta, \gamma)$ as $\delta=$ T-pred $(\gamma+1)$, so $\nu_{n} \leq \operatorname{crit}\left(E_{\gamma}\right)<$ strength $^{\mathscr{M}_{\alpha_{n}}}\left(E_{\alpha_{n}}\right)$, as desired.

Let $\delta_{n}=\mathrm{T}-\operatorname{pred}\left(\alpha_{n}+1\right)$ and $\gamma_{n}=\mathrm{T}-\operatorname{pred}\left(\beta_{n}+1\right)$ so that $\kappa_{n}=\operatorname{crit}\left(i_{\delta_{n}, b}\right)$ and $\nu_{n}=\operatorname{crit}\left(i_{\gamma_{n}, c}\right)$. The following claim is the key to the proof.

Claim. Let $t \in \operatorname{wfp}\left(\mathscr{M}_{b}\right) \cap \operatorname{wfp}\left(\mathscr{M}_{c}\right)$ and $t \in \operatorname{ran}\left(i_{\delta_{n}, b}\right) \cap \operatorname{ran}\left(i_{\gamma_{n}, c}\right)$. Let $\varphi$ be $\Sigma_{0}$.

(a) Let $\mu=\inf \left\{\kappa_{n}, \nu_{n}\right\}$ and $x \in V_{\mu}^{\mathscr{M}_{b}}$. If $\exists y \in V_{\theta}^{\mathscr{M}_{b}} \varphi(x, y, t)$, then $\exists y \in V_{\mu}^{\mathscr{M}_{b}} \varphi(x, y, t)$.

(b) Let $\mu=\inf \left\{\nu_{n}, \kappa_{n+1}\right\}$ and $x \in V_{\mu}^{\mathscr{M}_{b}}$. If $\exists y \in V_{\theta}^{\mathscr{M}_{b}} \varphi(x, y, t)$, then $\exists y \in V_{\mu}^{\mathscr{M}_{b}} \varphi(x, y, t)$.

Proof. We prove (a), the proof of (b) being nearly identical.

Because of the overlapping described above, we have, for all $m$,

$$
\begin{aligned}
& i_{\delta_{m}, b}\left(\kappa_{m}\right) \geq \operatorname{strength}^{\mathscr{M}_{\alpha_{m}}}\left(E_{\alpha_{m}}\right)>\nu_{m}, \\
& i_{\gamma_{m}, c}\left(\nu_{m}\right) \geq \operatorname{strength}^{\mathscr{M}_{\beta_{m}}}\left(E_{\beta_{m}}\right)>\kappa_{m+1} .
\end{aligned}
$$

Now let $m \geq n$, and suppose $\exists y \in V_{\nu_{m+1}}^{\mathscr{M}_{b}} \varphi(x, y, t)$. Setting $i=i_{\delta_{m+1}, b}$, we have, since $i\left(\kappa_{m+1}\right)>\nu_{m+1}$,

$$
\exists y \in V_{i\left(\kappa_{m+1}\right)}^{\mathscr{M}_{b}} \varphi(x, y, t) .
$$

Since $i(x)=x$ and $t \in \operatorname{ran}(i)$,

$$
\exists y \in V_{\kappa_{m+1}}^{\mathscr{M}_{\delta_{m+1}}} \varphi\left(x, y, i^{-1}(t)\right) .
$$

Since $\kappa_{m+1}=\operatorname{crit}(i)$,

$$
\exists y \in V_{\kappa_{m+1}}^{\mathscr{M}_{b}} \varphi(x, y, t) .
$$

Now we can repeat the argument on the $c$ side. Set $j=i_{\gamma_{m}, c}$; since $j\left(\nu_{m}\right)>$ $\kappa_{m+1}$ and $V_{\theta}^{\mathscr{M}_{b}}=V_{\theta}^{\mathscr{M}_{c}}$,

$$
\exists y \in V_{j\left(\nu_{m}\right)}^{\mathscr{M}_{c}} \varphi(x, y, t) .
$$


Since $j(x)=x$ and $t \in \operatorname{ran}(j)$,

$$
\exists y \in V_{\nu_{m}}^{\mathscr{M}_{\gamma_{m}}} \varphi\left(x, y, j^{-1}(t)\right) \text {. }
$$

Since $\nu_{m}=\operatorname{crit}(j)$,

$$
\exists y \in V_{\nu_{m}}^{\mathscr{M}_{c}} \varphi(x, y, t) .
$$

Repeating the argument one more time, we get $\exists y \in V_{\kappa_{m}}^{\mathscr{M}_{b}} \varphi(x, y, t)$.

The argument of the last paragraph required only that $m \geq n$. Thus it gives $\exists y \in V_{\nu_{n}}^{\mathscr{M}_{b}} \varphi(x, y, t)$ and $\exists y \in V_{\kappa_{n}}^{\mathscr{M}_{b}} \varphi(x, y, t)$. This proves the claim.

Now let $n$ be large enough that $f \in \operatorname{ran}\left(i_{\delta_{n}, b}\right) \cap \operatorname{ran}\left(i_{\gamma_{n}, c}\right)$. Suppose $\kappa_{n}<\nu_{n}$; the case $\nu_{n} \leq \kappa_{n}$ will be handled similarly. Notice that $f^{\prime \prime} \kappa_{n} \subseteq \kappa_{n}$ by the claim. Set $\tau=\inf \left\{\nu_{n}, \kappa_{n+1}\right\}$. So

$$
\kappa_{n}<\tau \leq \nu_{n}<\operatorname{strength}^{\mathscr{M}_{\alpha_{n}}}\left(E_{\alpha_{n}}\right) \text {. }
$$

By the claim, then, $f\left(\kappa_{n}\right)<\tau$. Now set

$$
F=E_{\alpha_{n}} \uparrow \tau=\left\langle\left(E_{\alpha_{n}}\right)_{a} \mid a \in[\tau]^{<\omega}\right\rangle .
$$

We shall show that, in $\mathscr{M}_{b}, F$ witnesses that $\theta$ is Woodin with respect to $f$.

We claim first that $F \in \mathscr{M}_{b}$ and strength ${ }^{\mathscr{M}_{b}}(F) \geq \tau$. For let $\xi+1$ be least in $c \backslash\left(\alpha_{n}+1\right)$, and let $\eta=\mathrm{T}$-pred $(\xi+1)$. Then

$$
\tau \leq \nu_{n} \leq \operatorname{crit}\left(E_{\xi}\right)<\rho(\eta, \xi) \leq \rho\left(\eta, \alpha_{n}\right) .
$$

As $F$ is essentially a member of $V_{\tau+1}^{\mathscr{M}_{\alpha_{n}}}, F \in \mathscr{M}_{\eta}$. But $\nu_{n}=\operatorname{crit}\left(i_{\eta, c}\right)$, so $i_{\eta, c}(F) \uparrow \tau=F$, so $F \in \mathscr{M}_{c}$, and hence $F \in \mathscr{M}_{b}$. Also, $\mathscr{M}_{\eta}$ agrees with $\mathscr{M}_{\alpha_{n}}$ through $\tau$, so $\mathscr{M}_{c}$ agrees with $\mathscr{M}_{\alpha_{n}}$ through $\tau$, so $\mathscr{M}_{b}$ agrees with $\mathscr{M}_{\alpha_{n}}$ through $\tau$. By Lemma 1.5, Ult $\left(\mathscr{M}_{b}, F\right)$ agrees with $\operatorname{Ult}\left(\mathscr{M}_{\alpha_{n}}, F\right)$ through $\tau$, and the latter agrees with $\operatorname{Ult}\left(\mathscr{M}_{\alpha_{n}}, E_{\alpha_{n}}\right)$ through $\tau$ as $\left|V_{\tau}\right|=\tau$ in $\operatorname{Ult}\left(\mathscr{M}_{\alpha_{n}}, E_{\alpha_{n}}\right)$. So $\operatorname{Ult}\left(\mathscr{M}_{b}, F\right)$ agrees with $\mathscr{M}_{b}$ through $\tau$; that is, strength $\mathscr{\mathscr { M }}_{b}(F) \geq \tau$.

Finally we must show that, in $\mathscr{M}_{b}, i_{F}(f)\left(\kappa_{n}\right)<\tau$. Let $\bar{f}=i_{\delta_{n}, b}^{-1}(f)$. Now $i_{\delta_{n}, b}=i_{\alpha_{n}+1, b} \circ i_{\delta_{n}, \alpha_{n}+1}$, and $\operatorname{crit}\left(i_{\alpha_{n}+1, b}\right)>\kappa_{n}$ from the definition of $\alpha_{n}$. Thus

$$
i_{\delta_{n}, b}(\bar{f})\left(\kappa_{n}\right) \geq i_{\delta_{n}, \alpha_{n}+1}(\bar{f})\left(\kappa_{n}\right) .
$$

But $f\left(\kappa_{n}\right)=i_{\delta_{n}, b}(\bar{f})\left(\kappa_{n}\right)<\tau$, and $i_{\delta_{n}, \alpha_{n}+1}=i_{E}^{\mathscr{P}}$, where $\mathscr{P}=\mathscr{M}_{\delta_{n}}$ and $E=E_{\alpha_{n}}$. Then $i_{E}^{\mathscr{P}}(\bar{f})\left(\kappa_{n}\right)<\tau$. By Lemma 1.5, $i_{E}^{\mathscr{M}_{b}}(\bar{f})\left(\kappa_{n}\right)<\tau$. As $F=$ $E \uparrow \tau, \quad i_{F}^{\mathscr{M}_{b}}(\bar{f})\left(\kappa_{n}\right)<\tau$, as desired.

Now suppose $\nu_{n} \leq \kappa_{n}$. Set $\tau=\inf \left\{\kappa_{n+1}, \nu_{n+1}\right\}$. We have $f^{\prime \prime} \nu_{n} \subseteq \nu_{n}$ and $f\left(\nu_{n}\right)<\tau$ by the claim. We can show that $E_{\beta_{n}} \uparrow \tau$ witnesses in $\mathscr{M}_{b}^{n}$ that $\theta$ is Woodin with respect to $f$ by repeating the argument just given. This completes the proof of the theorem.

We have at once 
Corollary 2.3. Let $\mathscr{T}$ be an iteration tree of limit length $\lambda$, and let $b$ and $c$ be distinct cofinal branches of $\mathscr{T}$. Let $\theta=\sup \left\{\rho^{\mathscr{T}}(\alpha, \lambda) \mid \alpha<\lambda\right\}$, and let $\alpha>\theta$ be such that $\alpha \in \operatorname{wfp}\left(\mathscr{M}_{b}\right) \cap \operatorname{wfp}\left(\mathscr{M}_{c}\right)$. Then $L_{\alpha}\left(V_{\theta}^{\mathscr{M}_{b}}\right) \vDash " \theta$ is Woodin".

Corollary 2.4. Let $\mathscr{M}=(M, \in, \delta)$ be a premouse such that $\mathscr{M} \vDash \forall \gamma \leq \delta$ “ $\gamma$ is not Woodin in $L\left(V_{\gamma}\right)$ ". Then every iteration tree on $\mathscr{M}$ has at most one cofinal wellfounded branch.

\section{Special Cases of iterability}

In the next section we shall prove a general theorem concerning the extendibility of countable iteration trees on countable elementary submodels of some $V_{\alpha}$. This section is devoted to the special case of trees of length $\leq \omega$. We have isolated this case to make the main ideas in the proof of $\S 4$ stand out and, less importantly, because the proof of $\S 4$ works only for plus two trees whereas here we can handle plus one trees.

In vague terms, our strategy runs as follows. Suppose $\mathscr{T}$ is a countable "bad" tree on a countable $\mathscr{M}_{0}$, and suppose $\pi_{0}: \mathscr{M}_{0} \rightarrow V_{\nu_{0}}^{\mathscr{P}_{0}}$, where $\mathscr{P}_{0}=V$. We define by induction on $\alpha$ premice $\mathscr{P}_{\alpha}$ and embeddings $\pi_{\alpha}: \mathscr{M}_{\alpha}^{\mathscr{T}} \rightarrow V_{\nu_{\alpha}}^{\mathscr{P}_{\alpha}}$. The $\mathscr{P}_{\alpha}$ 's are related to one another by something like the structure of $\mathscr{T}$ but with certain ultrapowers dictated by $\mathscr{T}$ approximated by internal ultrapowers. We can do this approximation because we maintain inductively that $\pi_{\alpha} \in \mathscr{P}_{\alpha}$; here we use $\mathscr{P}_{0}=V$ and our countability assumptions. Because enough ultrapowers are "internalized", the $\mathscr{P}_{\alpha}$ 's cannot absorb the badness of $\mathscr{T}$. And yet, via the $\pi_{\alpha}$ 's, they do. This is a contradiction.

In order to keep such an "enlargement" process going, we need some agreement among the $\pi_{\alpha}$ 's and $\mathscr{P}_{\alpha}$ 's, an agreement which reflects at least that part of the agreement among the $\mathscr{M}_{\alpha}^{\mathscr{T}}$ 's actually used in the construction of $\mathscr{T}$.

Definition 3.1. Let $\pi: \mathscr{M} \rightarrow \mathscr{P}$ and $\sigma: \mathscr{N} \rightarrow \mathscr{Q}$, where $\mathscr{M}, \mathscr{N}, \mathscr{P}$, and $\mathscr{Q}$ are premice. Let $\eta \in \mathrm{OR}^{\mathscr{M}} \cap \mathrm{OR}^{\mathscr{N}}$. We say that $\pi$ and $\sigma$ agree through $\eta$ iff

(a) $\mathscr{M}$ and $\mathscr{N}$ agree through $\eta$;

(b) $\pi\left(V_{\eta}^{\mathscr{M}}\right)=\sigma\left(V_{\eta}^{\mathscr{N}}\right)$, or, equivalently, $\pi(\eta)=\sigma(\eta)$ and $\mathscr{P}$ and $\mathscr{Q}$ agree through $\pi(\eta) ;$ and

(c) $\pi \uparrow V_{\eta}^{\mathscr{M}}=\sigma \mid V_{\eta}^{\mathcal{N}}$.

Definition 3.2. Let $\mathscr{T}$ be an iteration tree, and let $\beta+1<\operatorname{lh}(\mathscr{T})$. Then

$$
\mu^{\mathscr{T}}(\beta)=\sup \left\{\operatorname{crit}\left(E_{\gamma}^{\mathscr{T}}\right)+1 \mid \beta+1 \leq \gamma \wedge \mathrm{T}-\operatorname{pred}(\gamma+1) \leq \beta\right\},
$$

where $\sup \varnothing=0$.

One can think of $\mu^{\mathscr{T}}(\beta)$ as the amount of agreement between $\mathscr{M}_{\beta}^{\mathscr{T}}$ and $\mathscr{M}_{\beta+1}^{\mathscr{G}}$ actually used in constructing $\mathscr{T}$.

Definition 3.3. Let $\mathscr{T}$ be an iteration tree on $\mathscr{M}_{0}$, and let $\mu^{\mathscr{T}}(\beta) \leq g(\beta) \leq$ $\rho^{\mathscr{T}}(\beta, \beta+1)$ for all $\beta+1<\operatorname{lh}(\mathscr{T})$. A g-enlargement of $\left(\mathscr{T}, \mathscr{M}_{0}\right)$ is a 
sequence $\left\langle\left(\pi_{\alpha}, \mathscr{P}_{\alpha}, \nu_{\alpha}\right) \mid \alpha \leq \gamma\right\rangle$, where $\gamma<\operatorname{lh}(\mathscr{T})$, such that

(a) for all $\alpha \leq \gamma, \mathscr{P}_{\alpha}$ is a premouse, $\nu_{\alpha} \leq \mathrm{OR}^{\mathscr{P}_{\alpha}}$, and $\pi_{\alpha}: \mathscr{M}_{\alpha}^{\mathscr{T}} \rightarrow\left(V_{\nu_{\alpha}}^{\mathscr{P}}\right.$, $\in, \delta^{\mathscr{P}_{\alpha}}$ ) elementarily; and

(b) if $\alpha<\gamma$, then $\pi_{\alpha}$ agrees with $\pi_{\gamma}$ through $\inf \{g(\eta) \mid \alpha \leq \eta<\gamma\}$.

We allow the possibility $\nu_{\alpha}=\mathrm{OR}^{\mathscr{P}_{\alpha}}$ in Definition 3.3 ; in this case $V_{\nu_{\alpha}}^{\mathscr{P}_{\alpha}}=$ $\left|\mathscr{P}_{\alpha}\right|=$ universe of $\mathscr{P}_{\alpha}$.

It might seem more natural to require in (b) of 3.3 that if $\alpha<\beta \leq \gamma$, then $\pi_{\alpha}$ agrees with $\pi_{\beta}$ through $\inf \{g(\eta) \mid \alpha \leq \eta<\beta\}$. (Without this additional condition, a proper initial segment of an enlargement may not be an enlargement.) Unfortunately, in $\S 4$ we must deal with enlargements for which this additional condition fails.

Notice that condition (b) follows from: (i) if $\alpha+1 \leq \gamma$, then $\pi_{\alpha}$ agrees with $\pi_{\alpha+1}$ through $g(\alpha)$; and (ii) if $\alpha<\beta \leq \gamma$ and $\beta$ is a limit ordinal, then $\pi_{\alpha}$ agrees with $\pi_{\beta}$ through $\inf \{g(\eta) \mid \alpha \leq \eta<\beta\}$. In this section, only part (i) is relevant.

Notice also that if $\left\langle\left(\pi_{\alpha}, \mathscr{P}_{\alpha}, \nu_{\alpha}\right) \mid \alpha<\gamma+1\right\rangle$ is a $g$-enlargement of $\left(\mathscr{T}, \mathscr{M}_{0}\right)$ and $\alpha=\mathrm{T}$-pred $(\gamma+1)$, then $\operatorname{crit}\left(E_{\gamma}^{\mathscr{T}}\right)<\mu^{\mathscr{T}}(\beta) \leq g(\beta)$ whenever $\alpha \leq \beta<\gamma$, so $\pi_{\gamma}$ agrees with $\pi_{\alpha}$ through $\operatorname{crit}\left(E_{\gamma}^{\mathscr{T}}\right)+1$. This means that $\operatorname{Ult}\left(\mathscr{P}_{\alpha}, \pi_{\gamma}\left(E_{\gamma}^{\mathscr{T}}\right)\right)$, which is the natural candidate for $\mathscr{P}_{\gamma+1}$, makes sense.

It is easy to see that $\mathscr{T}$ is a plus $n$ tree if and only if, letting $g(\beta)$ be the $n$th successor ordinal $\geq \mu^{\mathscr{T}}(\beta)$, we have $g(\beta) \leq \rho^{\mathscr{T}}(\beta, \beta+1)$ for all $\beta$ such that $\beta+1<\ln (\mathscr{T})$.

Definition 3.4. Let $\mathscr{T}$ be a plus one iteration tree. A plus one enlargement of $\mathscr{T}$ is a $g$-enlargement, where $g(\beta)=1$ st successor ordinal $\geq \mu^{\mathscr{T}}(\beta)$ for all $\beta+1<\operatorname{lh}(\mathscr{T})$.

For the most part, we shall construct plus one enlargements. The next lemma records some elementary facts which will be useful in the construction of enlargements.

Lemma 3.5 (Shift lemma). Let $\pi: \overline{\mathscr{M}} \rightarrow \mathscr{M}$ and $\psi: \bar{N} \rightarrow\left(V_{\nu}^{\mathscr{N}}, \in, \delta^{\mathscr{N}}\right)$ be elementary, where $\overline{\mathscr{M}}, \mathscr{M}, \overline{\mathscr{N}}$, and $\mathscr{N}$ are premice and $\nu \leq \mathrm{OR}^{\mathscr{N}}$. Suppose $\pi$ and $\psi$ agree through $\bar{\kappa}+1, \bar{\kappa}<\inf \left(\delta^{\overline{\mathscr{M}}}, \delta^{\overline{\mathcal{N}}}\right)$, and $\overline{\mathscr{M}} \models$ " $\bar{E}$ is an extender, $\operatorname{crit}(\bar{E})=\bar{\kappa}$, and $\operatorname{strength}(\bar{E})=\bar{\rho} "$. Define $\sigma: \operatorname{Ult}(\overline{\mathcal{N}}, \bar{E}) \rightarrow$ $\left(V_{i(\nu)}^{\mathrm{Ult}(\mathcal{N}, E)}, \in, i\left(\delta^{\mathscr{N}}\right)\right)$, where $E=\pi(\bar{E})$ and $i=i_{E}^{\mathscr{N}}$, by

$$
\sigma\left([a, f]_{\bar{E}}^{\bar{N}}\right)=[\pi(a), \psi(f)]_{E}^{\mathscr{N}} .
$$

Then $\sigma$ is well defined and elementary, and the diagram

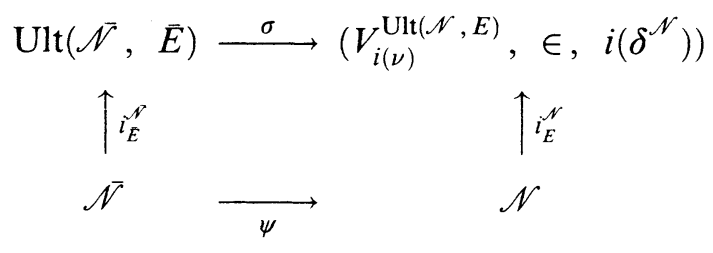


commutes. Moreover, $\sigma$ agrees with $\pi$ through $\bar{\rho}$.

Proof. We leave to the reader the proof that $\sigma$ is well defined and elementary and that the diagram commutes. Lemma 1.5 gives the desired agreement of models and that $i_{\bar{E}}^{\mathscr{\mu}}(\bar{\kappa})=i_{\bar{E}}^{\overline{\mathcal{K}}}(\bar{\kappa})$. We are left with showing that $\sigma$ and $\pi$ agree through $\bar{\rho}$. Since $\overline{\mathscr{M}}$ agrees with $\operatorname{Ult}(\overline{\mathscr{M}}, \bar{E})$ through $\bar{\rho}$ and $\operatorname{Ult}(\overline{\mathscr{M}}, \bar{E})$ agrees with $\operatorname{Ult}(\overline{\mathcal{N}}, \bar{E})$ through $i_{\bar{E}}^{\bar{N}}(\overline{\mathcal{K}})+1$, it is enough to show that $\sigma$ and $\pi$ agree on $V_{\eta+1}^{\mathrm{Ult}(\hat{\mathcal{N}}, E)}$, where $\eta=i_{\bar{E}}^{\widehat{N}}(\overline{\mathcal{K}})$. But every element of $V_{\eta+1}^{\mathrm{Ult}(\widehat{\mathcal{N}}, E)}$ is of the form $[a, f]_{\bar{E}}^{\tilde{N}}$, where $f \in V_{\tilde{\kappa}+1}^{\mathscr{N}}$, and for such elements we have

$$
\begin{aligned}
& \sigma\left([a, f]_{E}^{\mathscr{N}}\right)=[\pi(a), \psi(f)]_{E}^{\mathcal{N}}, \\
& =[\pi(a), \pi(f)]_{E}^{\mathcal{N}} \quad(\text { as } \pi(f)=\psi(f)), \\
& =[\pi(a), \pi(f)]_{E}^{\mathscr{M}} \quad(\text { as } \mathscr{M} \text { and } \mathscr{N} \text { agree through } \kappa+1), \\
& =\pi\left([a, f]_{E}^{\mathscr{M}}\right) \quad \text { (as } \pi \text { is elementary), } \\
& =\pi\left([a, f]_{\bar{E}}^{\widehat{N}}\right) \quad(\text { as } \mathscr{\mathscr { M }} \text { and } \overline{\mathscr{N}} \text { agree through } \bar{\kappa}+1),
\end{aligned}
$$

as desired.

We can construct a trivial enlargement of $\left(\mathscr{T}, \mathscr{M}_{0}\right)$ by merely carrying the structure of $\mathscr{T}$ from $\mathscr{M}_{0}$ over to some $\mathscr{P}$ into which $\mathscr{M}_{0}$ embeds. More precisely, suppose $\mathscr{T}$ is an iteration tree on $\mathscr{M}_{0}, \mathscr{P}$ is a premouse, and $\pi$ : $\mathscr{M}_{0} \rightarrow\left(V_{\nu}^{\mathscr{P}}, \in, \delta^{\mathscr{D}}\right)$ is elementary, with $\nu \leq \mathrm{OR}^{\mathscr{P}}$. We define a copied tree $\pi \mathscr{T}$ on $\mathscr{P}$ inductively as follows. Set $\mathscr{M}_{0}^{\pi \mathscr{T}}=\mathscr{P}$ and $\pi_{0}=\pi$ and $\nu_{0}=\nu$. Now suppose $1 \leq \gamma<\operatorname{lh}(\mathscr{T})$ and we have defined $\pi \mathscr{T}\left\lceil\gamma\right.$ and $\pi_{\alpha}, \nu_{\alpha}$ for $\alpha<\gamma$, so that

(a) $\pi \mathscr{T} \backslash \gamma$ is an iteration tree with tree order $\mathrm{T} \backslash \gamma$,

(b) $(\forall \delta<\gamma)\left\langle\left(\pi_{\alpha}, \mathscr{M}_{\alpha}^{\pi \mathscr{T}}, \nu_{\alpha}\right) \mid \alpha \leq \delta\right\rangle$ is an $f$-enlargement of $\left(\mathscr{T}, \mathscr{M}_{0}\right)$, where $f(\beta)=\rho^{\mathscr{g}}(\beta, \beta+1)$, and

(c) if $\beta<\gamma$ and $\alpha \mathrm{T} \beta$, then $i_{\alpha, \beta}^{\pi \mathscr{F}} \circ \pi_{\alpha}=\pi_{\beta} \circ i_{\alpha, \beta}^{\mathscr{I}}$; moreover, $\nu_{\beta}=$ $i_{\alpha, \beta}^{\pi \mathscr{T}}\left(\nu_{\alpha}\right)$.

Suppose first $\gamma$ is a limit. If the direct limit of the $\mathscr{M}_{\alpha}^{\pi \mathscr{G}}$, for $\alpha \mathrm{T} \gamma$, under the $i_{\alpha \beta}^{\pi \mathscr{T}}$ 's is illfounded, then we stop the construction (that is, $\pi \mathscr{T} \backslash \gamma+1$ is undefined). Otherwise, set

$$
\mathscr{M}_{\gamma}^{\pi \mathscr{T}}=\text { direct limit of } \mathscr{M}_{\alpha}^{\pi \mathscr{T}}, \alpha \mathrm{T} \gamma, \text { under the } i_{\alpha \beta}^{\pi \mathscr{T}} \text {. }
$$

$i_{\alpha \gamma}^{\pi \mathscr{T}}=$ the canonical embedding from $\mathscr{M}_{\alpha}^{\pi \mathscr{T}}$ into the direct limit, $\nu_{\gamma}=i_{\alpha \gamma}^{\pi \mathscr{T}}\left(\nu_{\alpha}\right)$ (for any and all $\alpha \mathrm{T} \gamma$ ), and

$$
\pi_{\gamma}\left(i_{\alpha \gamma}^{\mathscr{G}}(x)\right)=i_{\alpha \gamma}^{\pi \mathscr{F}}\left(\pi_{\alpha}(x)\right) \text {. }
$$

Notice the definition of $\pi_{\gamma}$ makes sense by (c). We leave it to the reader to verify that (a)-(c) continue to hold. (For (b), the key point is that if $\alpha<\gamma$, then for all sufficiently large $(\beta+1) \mathrm{T} \gamma, \quad \rho^{\mathscr{T}}(\alpha, \gamma)<\operatorname{crit}\left(E_{\beta}^{\mathscr{T}}\right)$.) 
Suppose $\gamma=\beta+1$, and let $\delta=\mathrm{T}-\operatorname{pred}(\gamma)$. Notice that $\operatorname{Ult}\left(\mathscr{M}_{\delta}^{\pi \mathscr{T}}, \pi_{\beta}\left(E_{\beta}^{\mathscr{T}}\right)\right)$ makes sense by (b) and the fact $\operatorname{crit}\left(E_{\beta}^{\mathscr{T}}\right)<\rho^{\mathscr{T}}(\delta, \beta)$. If $\operatorname{Ult}\left(\mathscr{M}_{\delta}^{\pi \mathscr{T}}, \pi_{\beta}\left(E_{\beta}^{\mathscr{T}}\right)\right)$ is illfounded, then again we stop the construction and leave $\pi \mathscr{T} \uparrow \gamma+1$ undefined. Otherwise, set

$$
\begin{aligned}
\mathscr{M}_{\gamma}^{\pi \mathscr{T}} & =\operatorname{Ult}\left(\mathscr{M}_{\delta}^{\pi \mathscr{T}}, \pi_{\beta}\left(E_{\beta}^{\mathscr{T}}\right)\right) \\
i_{\delta \gamma}^{\pi \mathscr{T}} & =\text { canonical embedding into the ultrapower, } \\
\nu_{\gamma} & =i_{\delta \gamma}^{\pi \mathscr{T}}\left(\nu_{\delta}\right),
\end{aligned}
$$

and, as in the shift lemma,

$$
\pi_{\gamma}\left([a, f]_{E}^{\bar{N}}\right)=\left[\pi_{\beta}(a), \pi_{\delta}(f)\right]_{E}^{\mathscr{N}},
$$

where $\overline{\mathcal{N}}=\mathscr{M}_{\delta}^{\mathscr{T}}, \bar{E}=E_{\beta}^{\mathscr{T}}, \mathscr{N}=\mathscr{M}_{\delta}^{\pi \mathscr{T}}$, and $E=\pi_{\beta}\left(E_{\beta}^{\mathscr{T}}\right)$. Using the shift lemma, one easily verifies that (a)-(c) continue to hold.

Definition 3.6. If $\mathscr{T}$ is an iteration tree on $\mathscr{M}_{0}$ and $\pi: \mathscr{M}_{0} \rightarrow\left(V_{\nu}^{\mathscr{P}}, \in, \delta^{\mathscr{P}}\right)$ is elementary, where $\mathscr{P}$ is a premouse and $\nu \leq \mathrm{OR}^{\mathscr{P}}$, then $\pi \mathscr{T}$ is the copied tree on $\mathscr{P}$ described above.

We now show that finite iteration trees on $V$ can be freely extended. The proof involves a nontrivial enlargement of a bad tree.

Theorem 3.7. Let $\mathscr{T}$ be an iteration tree of length $\beta+1$, where $\beta<\omega$. Suppose $\mathscr{M}_{0}^{\mathscr{T}}$ is elementarily embeddable into $\left(V_{\nu}, \in, \delta\right)$, for some $\nu, \delta$. Let $\mathscr{M}_{\beta}^{\mathscr{T}} \models$ “ $E$ is an extender with critical point $\kappa$ ", and suppose $\kappa<\rho^{\mathscr{T}}(\alpha, \beta)$, where $\alpha \leq \beta$. Then $\operatorname{Ult}\left(\mathscr{M}_{\alpha}^{\mathscr{T}}, E\right)$ is wellfounded.

Proof. Suppose not, and let $\beta<\omega$ be least such that the theorem fails for some iteration tree of length $\beta+1$. The Löwenheim-Skolem theorem yields such a failure for a tree on a countable base model. For let $\mathscr{U}$ be an iteration tree on $\mathscr{M}_{0}^{\mathscr{U}}$ of length $\beta+1$, let $\pi: \mathscr{M}_{0}^{\mathscr{U}} \rightarrow\left(V_{\nu}, \in, \delta\right)$ elementarily, and suppose $\mathscr{M}_{\beta}^{\mathscr{U}} \vDash{ }^{\prime} E^{\prime}$ is an extender with critical point $\kappa^{\prime} ", \kappa^{\prime}<\rho^{\mathscr{U}}(\alpha, \beta)$ where $\alpha \leq \beta$, and $\operatorname{Ult}\left(\mathscr{M}_{\alpha}^{\mathscr{U}}, E^{\prime}\right)$ is illfounded. Let $\eta$ be a limit ordinal such that $\mathscr{U}, \mathscr{M}_{0}^{\mathscr{U}} \in V_{\eta}$. Forming a Skolem hull and collapsing, we obtain a countable transitive $N$ and elementary embedding $\sigma: N \rightarrow V_{\eta}$ such that $E^{\prime}, \mathscr{U}, \mathscr{M}_{0}^{\mathscr{U}} \in$ $\operatorname{ran}(\sigma)$. Let $\sigma(\mathscr{T})=\mathscr{U}, \sigma\left(\mathscr{M}_{0}^{\mathscr{T}}\right)=\mathscr{M}_{0}^{\mathscr{U}}, \sigma(E)=E^{\prime}$, and $\sigma(\kappa)=\kappa^{\prime} . \mathscr{T}$ is an iteration tree of length $\beta+1$ on $\mathscr{M}_{0}^{\mathscr{T}}$ witnessing the failure of Theorem 3.7; notice here that $\pi \circ\left(\sigma \mid \mathscr{M}_{0}^{\mathscr{T}}\right)$ maps $\mathscr{M}_{0}^{\mathscr{T}}$ into $\left(V_{\nu}, \in, \delta\right)$ elementarily.

We now construct a $g$-enlargement of $\mathscr{T}$, where $g$ is determined as follows. Set

$$
g(\gamma)= \begin{cases}\mu^{\mathscr{T}}(\gamma) & \text { if } \gamma<\alpha, \\ \max \left(\mu^{\mathscr{T}}(\gamma), \kappa+1\right) & \text { if } \alpha \leq \gamma<\beta .\end{cases}
$$

As $\kappa<\rho^{\mathscr{T}}(\alpha, \beta)=\inf \left\{\rho^{\mathscr{T}}(\gamma, \gamma+1) \mid \alpha \leq \gamma<\beta\right\}$, we have $\mu^{\mathscr{T}}(\gamma) \leq g(\gamma) \leq$ $\rho^{\mathscr{T}}(\gamma, \gamma+1)$ for all $\gamma<\beta$. 
We define our $g$-enlargement $\left\langle\left(\pi_{i}, \mathscr{P}_{i}, \nu_{i}\right) \mid i \leq \beta\right\rangle$ by induction on $i$. We maintain inductively that all its initial segments are also $g$-enlargements. Let $\eta>\nu$ be such that $\left(V_{\eta}, \in, \delta\right)$ is a premouse, and let

$$
\begin{aligned}
\mathscr{P}_{0} & =\left(V_{\eta}, \in, \delta\right), \\
\nu_{0} & =\nu, \\
\pi_{0} & =\pi \circ\left(\sigma \nmid \mathscr{M}_{0}^{\mathscr{T}}\right) .
\end{aligned}
$$

Now suppose we are given $\left\langle\left(\pi_{i}, P_{i}, \nu_{i}\right) \mid i \leq \gamma\right\rangle$, where $\gamma<\beta$. Suppose also that $\pi_{\gamma} \in \mathscr{P}_{\gamma}$ and that the $\mathscr{P}_{i}$ 's are the models of an iteration tree of length $\gamma+1$. (We shall maintain this inductively.) Let $\xi=\mathrm{T}$-pred $(\gamma+1)$. Now if $\xi<\gamma$, then $\operatorname{crit}\left(E_{\gamma}^{\mathscr{T}}\right)+1 \leq \inf \{g(\eta) \mid \xi \leq \eta<\gamma\}$; thus in any case $\pi_{\xi}$ and $\pi_{\gamma}$ agree through $\operatorname{crit}\left(E_{\gamma}^{\mathscr{T}}\right)+1$. Note also $\operatorname{crit}\left(E_{\gamma}^{\mathscr{T}}\right)<\rho^{\mathscr{T}}(\xi, \xi+1)<\delta^{\mathscr{P}_{\xi}}$. We are therefore in a position to apply the shift lemma: set

$$
\begin{aligned}
\mathscr{P}_{\gamma+1} & =\operatorname{Ult}\left(\mathscr{P}_{\xi}, \pi_{\gamma}\left(E_{\gamma}^{\mathscr{T}}\right)\right), \\
\nu_{\gamma+1} & =i\left(\nu_{\xi}\right), \quad \text { where } i: \mathscr{P}_{\xi} \rightarrow \mathscr{P}_{\gamma+1} \text { canonically, }
\end{aligned}
$$

and

$$
\tau\left([a, f]_{E_{\gamma}^{\mathcal{G}}}^{\mathscr{M}_{\mathcal{G}}^{\mathcal{G}}}\right)=\left[\pi_{\gamma}(a), \pi_{\xi}(f)\right]_{\pi_{\gamma}\left(E_{\gamma}^{\mathcal{G}}\right)}^{\mathscr{P}_{\xi}} .
$$

Thus $\tau: \mathscr{M}_{\gamma+1}^{\mathscr{T}} \rightarrow\left(V_{\nu_{\gamma+1}}^{\mathscr{P}_{\gamma+1}}, \in, \delta^{\mathscr{P}_{\gamma+1}}\right)$ elementarily, and $\tau$ agrees with $\pi_{\gamma}$ through $\rho^{\mathscr{T}}(\gamma, \gamma+1)$. Notice that because the $\mathscr{P}_{i}$ 's are the models of an iteration tree of length $\gamma+1<\beta+1$ and $\beta$ was chosen least, $\mathscr{P}_{\gamma+1}$ is wellfounded.

Let $\psi=\tau \uparrow V_{g(\gamma)}^{\mathscr{M}_{\gamma+1}}=\pi_{\gamma} \uparrow V_{g(\gamma)}^{\mathscr{M}_{\gamma+1}}$. As $\pi_{\gamma} \in \mathscr{P}_{\gamma}, \psi \in \mathscr{P}_{\gamma}$. On the other hand, $g(\gamma)$ is a successor ordinal (note $\mu^{\mathscr{T}}(\gamma)$ is a successor because $\mathscr{T}$ is finite), and letting $\tau(g(\gamma))=\eta+1=\pi_{\gamma}(g(\gamma))$, we have that $\psi$ is a countable subset of $V_{\eta+1}^{\mathscr{P}_{\gamma}}$ belonging to $\mathscr{P}_{\gamma}$. Thus $\psi$ can be coded by a member of $V_{\eta+1}^{\mathscr{P}_{\gamma}}$. But $\mathscr{P}_{\gamma}$ agrees with $\mathscr{P}_{\gamma+1}$ through $\eta+1$, so that $\psi \in \mathscr{P}_{\gamma+1}$.

It follows that there is a tree (set of finite sequences closed under initial segment) $\mathscr{U} \in \mathscr{P}_{\gamma+1}$ such that the infinite branches of $\mathscr{U}$ are precisely those elementary embeddings $\pi: \mathscr{M}_{\gamma+1}^{\mathscr{T}} \rightarrow\left(V_{\nu_{\gamma+1}}^{\mathscr{P}_{\gamma+1}}, \in \delta^{\mathscr{P}_{\gamma+1}}\right)$ such that $\pi \uparrow V_{g(\gamma)}^{\mathscr{M}_{\gamma+1}}=\psi$. $\mathscr{U}$ has an infinite branch, namely, $\tau$. Since $\mathscr{P}_{\gamma+1}$ is a wellfounded model of enough set theory, there is a branch of $\mathscr{U}$ belonging to $\mathscr{P}_{\gamma+1}$; let $\pi_{\gamma+1}$ be such a branch.

Since $\pi_{\gamma+1}$ agrees with $\psi$, and hence $\pi_{\gamma}$, through $g(\gamma)$, we have that $\left\langle\left(\pi_{i}, \mathscr{P}_{i}, \nu_{i}\right) \mid i \leq \gamma+1\right\rangle$ is a $g$-enlargement of $\mathscr{T}$. Clearly, $\pi_{\gamma+1} \in \mathscr{P}_{\gamma+1}$, and the $\mathscr{P}_{i}, i \leq \gamma+1$, are the models of an iteration tree. This completes the defintion of $\left\langle\left(\pi_{i}, \mathscr{P}_{i}, \nu_{i}\right) \mid i \leq \beta\right\rangle$.

Now let $F=\pi_{\beta}(E)$ and $\lambda=\operatorname{crit}(F)=\pi_{\beta}(\kappa)$. Pick $f_{e}, a_{e}$ for $e \in \omega$ such that

$$
\left[a_{e+1}, f_{e+1}\right]_{E}^{\mathscr{M}_{\alpha}^{\mathcal{G}}} \in\left[a_{e}, f_{e}\right]_{E}^{\mathscr{M}_{\alpha}^{\mathcal{G}}}
$$


for all $e \in \omega$, and $\left\langle\left(f_{e}, a_{e}\right) \mid e \in \omega\right\rangle \in \mathscr{P}_{\beta}$. We may assume $a_{e} \subseteq a_{e+1}$ for all $e$.

If $s \in[\mathrm{OR}]^{n}$, where $n=|a|$ and $a \subseteq \mathrm{OR}$, and $b \subseteq a$, set $s_{a, b}=\pi^{\prime \prime} b$, where $\pi: a \rightarrow s$ is the unique order-preserving map. For $s \in[\kappa]^{n}$, with $n=\left|a_{e+1}\right|$, put

$$
s \in A_{e} \Leftrightarrow f_{e+1}(s) \in f_{e}\left(s_{a_{e+1}, a_{e}}\right) .
$$

We have that $A_{e} \in E_{a_{e+1}}$, for all $e$.

Now let

$$
\begin{aligned}
& B_{e}=\pi_{\beta}\left(A_{e}\right)=\pi_{\alpha}\left(A_{e}\right), \\
& b_{e}=\pi_{\beta}\left(a_{e}\right) .
\end{aligned}
$$

Then $B_{e} \in F_{b_{e+1}}$ for all $e$. Moreover, $\left\langle B_{e} \mid e \in w\right\rangle$ and $\left\langle b_{e} \mid e \in w\right\rangle$ belong to $\mathscr{P}_{\beta}$, since $\pi_{\beta} \in \mathscr{P}_{\beta}$. (This was the point of the construction.) As $\mathscr{P}_{\beta} \vDash " F$ is an extender", we can find (in $\mathscr{P}_{\beta}$ ) an order-preserving map $g: \bigcup_{e} b_{e} \rightarrow \lambda$ such that $g^{\prime \prime} b_{e+1} \in B_{e}$ for all $e$.

Notice that for each $e$, the map $s \mapsto s_{a_{e+1}, a_{e}}$ on $[\kappa]^{\left|a_{e+1}\right|}$ is definable in $\mathscr{M}_{\alpha}^{\mathscr{T}}$, and its image under $\pi_{\alpha}$ is the map $s \mapsto s_{b_{e+1}, b_{e}}$ on $[\lambda]^{\left|b_{e+1}\right|}$. As $\pi_{\alpha}$ is elementary, we have

$$
\pi_{\alpha}\left(f_{e+1}\right)(s) \in \pi_{\alpha}\left(f_{e}\right)\left(s_{b_{e+1}, b_{e}}\right)
$$

for all $s \in B_{e}$. But $\left(g^{\prime \prime} b_{e+1}\right)_{b_{e+1}, b_{e}}=g^{\prime \prime} b_{e}$, and thus

$$
\pi_{\alpha}\left(f_{e+1}\right)\left(g^{\prime \prime} b_{e+1}\right) \in \pi_{\alpha}\left(f_{e}\right)\left(g^{\prime \prime} b_{e}\right)
$$

for all $e$. So we have an infinite descending $\in$-chain in $\mathscr{P}_{\alpha}$, as contradiction.

We turn now to the extendibility of trees of length $\omega$, that is, to the existence of cofinal wellfounded branches in such trees.

Definition 3.8. Let $\mathscr{T}$ be an iteration tree of length $\omega$ on $\mathscr{M}$, with tree order $\mathrm{T}$. We say $\mathscr{T}$ is continuously illfounded (on $\mathscr{M}$ ) iff there are ordinals $\theta_{n} \in \mathscr{M}_{n}^{\mathscr{T}}$, for $n<\omega$, such that $i_{n m}^{\mathscr{T}}\left(\theta_{n}\right)>\theta_{m}$ whenever $n \mathrm{~T} m$.

Theorem 3.9. Let $\mathscr{M}$ be a premouse which is elementarily embeddable into $\left(V_{\nu}, \in, \delta\right)$ for some $\nu, \delta$. Then there is no continuously illfounded plus one iteration tree of length $\omega$ on $\mathscr{M}$.

Proof. Suppose not. Let

$$
\pi_{0}: \mathscr{M} \rightarrow\left(V_{\nu_{0}}, \in, \delta\right)
$$

Suppose $\mathscr{T}$ is a length $\omega$ plus one iteration tree on $\mathscr{M}=\mathscr{M}_{0}^{\mathscr{T}}$ and that $\theta_{n} \in \mathrm{OR} \cap \mathscr{M}_{n}^{\mathscr{T}}$ for all $n$, with $i_{n m}^{\mathscr{T}}\left(\theta_{n}\right)>\theta_{m}$ whenever $n \mathrm{~T} m$. By taking a Skolem hull, we can arrange that $\mathscr{M}$ is countable, so we assume this. 
Let $\eta>\nu_{0}$ be such that $\left(V_{\eta}, \in, \delta\right)$ is a premouse and $\left\{\gamma \mid \nu_{0}<\gamma<\right.$ $\eta \wedge\left(V_{\gamma}, \in, \delta\right)$ is a premouse $\}$ has order type $\pi_{0}\left(\theta_{0}\right)$. Set

$$
\mathscr{P}_{0}=\left(V_{\eta}, \in, \delta\right) \text {. }
$$

Now suppose we have defined $\left\langle\left(\pi_{i}, \mathscr{P}_{i}, \nu_{i}\right) \mid i \leq \gamma\right\rangle$, where $\gamma<\omega$, so that

(i) $\left\langle\left(\pi_{i}, \mathscr{P}_{i}, \nu_{i}\right) \mid i \leq \gamma\right\rangle$ is a plus one enlargement of $\mathscr{T}$,

(ii) $\mathscr{P}_{i+1} \in \mathscr{P}_{i}$ for all $i<\gamma$,

(iii) $\pi_{\gamma} \in \mathscr{P}_{\gamma}$,

(iv) $\left\{\eta \mid \nu_{\gamma}<\eta<\left(\mathrm{OR} \cap \mathscr{P}_{\gamma}\right)\right.$ and $\left(V_{\eta}^{\mathscr{P}_{\gamma}}, \in, \delta^{\mathscr{P}_{\gamma}}\right)$ is a premouse $\}$ has order type $\pi_{\gamma}\left(\theta_{\gamma}\right)$.

We define $\left(\pi_{\gamma+1}, \mathscr{P}_{\gamma+1}, \nu_{\gamma+1}\right)$ so as to preserve (i)-(iv).

Let $\xi=\mathrm{T}-\operatorname{pred}(\gamma+1)$, and set

$$
\mathscr{Q}=\operatorname{Ult}\left(\mathscr{P}_{\xi}, \pi_{\gamma}\left(E_{\gamma}^{\mathscr{T}}\right)\right)
$$

Notice that $\pi_{\xi}$ agrees with $\pi_{\gamma}$ sufficiently that the ultrapower makes sense. Also, $\mathscr{Q}$ is wellfounded. This is clear if $\xi=\gamma$. If $\xi<\gamma$, then $\mathscr{P}_{\gamma} \in \mathscr{P}_{\xi}$, so $\pi_{\gamma}\left(E_{\gamma}^{\mathscr{T}}\right) \in \mathscr{P}_{\xi}$. If $\mathscr{P}_{\xi} \vDash$ “ $\pi_{\gamma}\left(E_{\gamma}^{\mathscr{T}}\right)$ is not $\omega$-complete", then there is a sequence $\left\langle a_{n}, X_{n} \mid n<\omega\right\rangle \in \mathscr{P}_{\xi}$ such that $X_{n} \in\left(\pi_{\gamma}\left(E_{\gamma}^{\mathscr{T}}\right)\right)_{a_{n}}$ and there is no $g: \bigcup_{n \in w} a_{n} \rightarrow \operatorname{crit}\left(\pi_{\gamma}\left(E_{\gamma}^{\mathscr{T}}\right)\right)$ such that $g^{\prime \prime} a_{n} \in X_{n}$ for all $n$. Now $\mathscr{P}_{\gamma}$ agrees with $\mathscr{P}_{\xi}$ sufficiently that $\left\langle X_{n} \mid n<\omega\right\rangle \in \mathscr{P}_{\gamma}$. Let $t: \bigcup_{n<\omega} a_{n} \stackrel{\text { onto }}{\longrightarrow} \alpha$ be order preserving, where $\alpha<\omega_{1}$, and $\bar{a}_{n}=t^{\prime \prime} a_{n}$. Then $\left\langle\bar{a}_{n} \mid n<\omega\right\rangle \in \mathscr{P}_{\gamma}$ since $\mathscr{P}_{\gamma}$ agrees with $\mathscr{P}_{0}$ through $\omega+1$. Let $i: \mathscr{P}_{\gamma} \rightarrow \operatorname{Ult}\left(\mathscr{P}_{\gamma}, \pi_{\gamma}\left(E_{\gamma}^{\mathscr{g}}\right)\right)$ be the canonical embedding. Letting $g=t^{-1}$, we have $g^{\prime \prime} \bar{a}_{n}=a_{n} \in i\left(X_{n}\right)$ for all $n$. As $\operatorname{Ult}\left(\mathscr{P}_{\gamma}, \pi_{\gamma}\left(E_{\gamma}^{\mathscr{T}}\right)\right)$ is wellfounded, there is by absoluteness a function $g \in \operatorname{Ult}\left(\mathscr{P}_{\gamma}, \pi_{\gamma}\left(E_{\gamma}^{\mathscr{T}}\right)\right)$ such that $g^{\prime \prime} \bar{a}_{n} \in i\left(X_{n}\right)$ for all $n$. By the elementary of $i$, there is a $g \in \mathscr{P}_{\gamma}$ such that $g^{\prime \prime} \bar{a}_{n} \in X_{n}$ for all $n$. But there is by hypothesis no such $g$, contradiction. Thus $\mathscr{Q}$ is wellfounded.

Let $i: \mathscr{P}_{\xi} \rightarrow \mathscr{Q}$ be the canonical embedding. From the shift lemma we have $\tau: \mathscr{M}_{\gamma+1}^{\mathscr{T}} \rightarrow\left(V_{i\left(\nu_{\xi}\right)}^{\mathscr{Q}}, \in, \delta^{\mathscr{Q}}\right)$ which agrees with $\pi_{\gamma}$ through $\rho^{\mathscr{T}}(\gamma, \gamma+1)$. Now let $\eta+1$ be the first successor ordinal $\geq \mu^{\mathscr{T}}(\gamma)$; as $\mathscr{T}$ is a plus one tree, $\eta+1 \leq \rho^{\mathscr{T}}(\gamma, \gamma+1)$. Let $\psi=\tau \uparrow V_{\eta+1}^{\mathscr{M}_{\gamma+1}^{\mathscr{g}}}=\pi_{\gamma} \uparrow V_{\eta+1}^{\mathscr{M}_{\gamma+1}^{\mathscr{T}}}$. Since $\psi$ is coded by a member of $V_{\tau(\eta)+1}^{\mathscr{P}_{\gamma}}=V_{\tau(\eta)+1}^{\mathscr{Q}}, \psi \in \mathscr{Q}$. As in the proof of Theorem 3.7 we can form in $\mathscr{Q}$ a tree (set of finite sequences closed under initial segment) whose infinite branches are precisely those elementary embeddings $\sigma: \mathscr{M}_{\gamma+1}^{\mathscr{T}} \rightarrow$ $\left(V_{i\left(\nu_{\xi}\right)}^{\mathscr{Q}}, \in, \delta^{\mathscr{Q}}\right)$ such that $\psi \subseteq \sigma$ and $\sigma\left(\theta_{\gamma+1}\right)=\tau\left(\theta_{\gamma+1}\right)$. This tree has a branch, namely $\tau$, so it has a branch $\sigma \in \mathscr{Q}$.

We have done what we need to do to propagate induction hypothesis (iii). We now turn to (ii). Notice that

$$
\sigma\left(\theta_{\gamma+1}\right)=\tau\left(\theta_{\gamma+1}\right)<\tau\left(i_{\xi, \gamma+1}^{\mathscr{G}}\left(\theta_{\xi}\right)\right)=i\left(\pi_{\xi}\left(\theta_{\xi}\right)\right) .
$$


It is a first-order property of $\mathscr{P}_{\xi}$ that there are, in order type, $\pi_{\xi}\left(\theta_{\xi}\right)$ many ordinals $\nu$ such that $\nu_{\xi}<\nu<\mathrm{OR}^{\mathscr{P}_{\xi}}$ and $\left(V_{\nu}^{\mathscr{P}_{\xi}}, \in, \delta^{\mathscr{P}_{\xi}}\right)$ is a premouse. This property therefore carries over to $\mathscr{Q}$, so that there are, in order type, $i\left(\pi_{\xi}\left(\theta_{\xi}\right)\right)$ many ordinals $\nu$ such that $i\left(\nu_{\xi}\right)<\nu<\mathrm{OR}^{\mathscr{Q}}$ and $\left(V_{\nu}^{\mathscr{Q}}, \in, \delta^{\mathscr{Q}}\right)$ is a premouse. It follows that there is a $\sigma\left(\theta_{\gamma+1}\right)$ th such ordinal; call it $\mu$.

Working in $\mathscr{Q}$ we can find a set $X$, with $\operatorname{card}^{\mathscr{Q}}(X)=\operatorname{card}^{\mathscr{Q}}\left(V_{\sigma(\eta)+1}^{\mathscr{Q}}\right)$, such that

$$
\left(X, \in, \delta^{\mathscr{Q}}\right) \prec\left(V_{\mu}^{\mathscr{Q}}, \in, \delta^{\mathscr{Q}}\right)
$$

and

$$
V_{\sigma(\eta)+1}^{\mathscr{Q}} \cup\left\{\sigma, \quad i\left(\nu_{\xi}\right)\right\} \subseteq X .
$$

Let $\pi:(X, \in) \cong(P, \in)$, where $P$ is transitive, and set

$$
\begin{aligned}
\mathscr{P}_{\gamma+1} & =\left(P, \in, \pi\left(\delta^{\mathscr{Q}}\right)\right), \\
\nu_{\gamma+1} & =\pi\left(i\left(\nu_{\xi}\right)\right), \\
\pi_{\gamma+1} & =\pi(\sigma) .
\end{aligned}
$$

(Notice $\mathscr{M}_{\gamma+1}^{\mathscr{T}} \in V_{\sigma(\eta)+1}^{\mathscr{Q}} \subseteq X$, so $\pi_{\gamma+1}=\pi \circ \sigma$ has domain $\mathscr{M}_{\gamma+1}^{\mathscr{T}}$.) One can check easily that our induction hypotheses (i), (iii), and (iv) continue to hold. As for (ii), since $\operatorname{card}^{\mathscr{Q}}(X)=\operatorname{card}^{\mathscr{Q}}\left(V_{\sigma(\eta)+1}^{\mathscr{Q}}\right), P$ can be coded by a set $A \in \mathscr{Q}$ such that $A \subseteq V_{\sigma(\eta)+1}^{\mathscr{Q}}$. But $\mathscr{Q}=\operatorname{Ult}\left(\mathscr{P}_{\xi}, \pi_{\gamma}\left(E_{\gamma}^{\mathscr{T}}\right)\right)$ agrees with $\operatorname{Ult}\left(\mathscr{P}_{\gamma}, \pi_{\gamma}\left(E_{\gamma}^{\mathscr{T}}\right)\right)$ through $i(\kappa)$, where $\kappa=\operatorname{crit}\left(\pi_{\gamma}\left(E_{\gamma}^{\mathscr{T}}\right)\right)$, and hence through $\pi_{\gamma}(\eta)+2=\sigma(\eta)+2$. So $A \in \operatorname{Ult}\left(\mathscr{P}_{\gamma}, \pi_{\gamma}\left(E_{\gamma}^{\mathscr{T}}\right)\right)$, so $A \in \mathscr{P}_{\gamma}$, so $\mathscr{P}_{\gamma+1} \in \mathscr{P}_{\gamma}$.

This completes the inductive definition of $\left\langle\left(\pi_{i}, \mathscr{P}_{i}, \nu_{i}\right) \mid i<\omega\right\rangle$. Since $\mathscr{P}_{i+1} \in \mathscr{P}_{i}$ for all $i$, we have a contradiction.

By an alternating chain we mean an iteration tree $\mathscr{T}$ of length $\omega$ whose associated tree order T is defined by: $n \mathrm{~T} m$ iff $[(n=0 \wedge m>0) \vee n \equiv m(\bmod$ 2)]. Alternating chains figure importantly in the determinacy proof of [MS]. Clearly, every alternating chain is a plus one iteration tree which is the union of two cofinal branches. Theorem 3.9 therefore implies that every alternating chain on a premouse $\mathscr{M} \prec\left(V_{\alpha}, \in, \delta\right)$ has a cofinal wellfounded branch.

We do not know whether every plus one iteration tree on $V$ of length $\omega$ has a cofinal wellfounded branch. We discuss this question further in $\S 5$. From Theorem 3.9 we do get

Corollary 3.10. Let $\mathscr{M}$ be a premouse elementary embeddable into $\left(V_{\alpha}, \in, \delta\right)$ for some $\alpha, \delta$, and let $\mathscr{T}$ be a plus one iteration tree of length $\omega$ on $\mathscr{M}$. Then $\mathscr{T}$ has a cofinal branch.

Proof. Let $\pi: \mathscr{M} \rightarrow \mathscr{P}=\left(V_{\alpha}, \in, \delta\right)$ be elementary. So $\pi \mathscr{T}$ is a plus one iteration tree of length $\omega$ on $\mathscr{P}$. Suppose $\mathscr{T}$ has no cofinal branch; then the converse $\check{\mathrm{T}}$ of $\mathrm{T}$ is wellfounded. (Here $n \check{\mathrm{T}} m$ iff $m \mathrm{~T} n$.) Letting $\theta_{n}=$ rank of $n$ in $\check{\mathrm{T}}$, for $n \in \omega$, we have $n \check{\mathrm{T}} m \Rightarrow \theta_{n}>\theta_{m}$. But $\omega_{1}<\mathrm{OR}^{\mathscr{P}}$, 
so $n \check{\mathrm{T}} m \Rightarrow i^{\pi \mathscr{T}}\left(\theta_{n}\right)>\theta_{m}$. Thus $\pi \mathscr{T}$ is continuously illfounded, contrary to 3.9 .

Mitchell [Mil] has defined a relation $\triangleleft$ on extenders which in some sense measures their strength. For $E$ and $F$ extenders (over $V$ ), we have $E \triangleleft$ $F$ iff $E \in \operatorname{Ult}(V, F)$. (This relation is now known as the Mitchell order, although it may not be transitive.) Mitchell showed in [Mi1] that the restriction $\triangleleft$ to normal measures (that is, $(\kappa, \kappa+1)$ extenders) is wellfounded. Corollary 3.10 comes close to generalizing this result to arbitrary extenders. For suppose $E_{i+1} \triangleleft E_{i}$ for all $i \in \omega$, and let $\mathrm{T}$ be the tree order on $\omega$ given by: $n \mathrm{~T} m$ iff $(n=0 \wedge m>0)$. Let $\mathscr{T}=\left\langle\mathrm{T},\left\langle E_{i} \mid i \in \omega\right\rangle\right\rangle$. Then $\mathscr{T}$ is an iteration tree of length $\omega$ on $V$ having no cofinal branch, and we have a contradiction to Corollary 3.10 if $\mathscr{T}$ is a plus one tree.

Notice that if $E_{i+1} \triangleleft E_{i}$ for all $i \in \omega$ and there is a fixed $\kappa$ such that $\operatorname{crit}\left(E_{i}\right)=\kappa$ for all $i$, then the tree $\mathscr{T}$ described in the previous paragraph is a plus one tree. Thus there is no infinite descending $\triangleleft$-chain whose extenders all have the same critical point. The next corollary is an application of this fact. If $E$ and $F$ are extenders with critical point $\kappa$ and $\pi: \operatorname{lh}(E) \rightarrow \operatorname{lh}(F)$ is order preserving and $\pi(\kappa)=\kappa$, then we say that $E$ is a subextender of $F$ via $\pi$ iff $\forall a \in[\operatorname{lh}(E)]^{<\omega}\left(E_{a}=F_{\pi^{\prime \prime} a}\right)$.

Corollary 3.11. Let $E$ be an extender; then $E$ is not a subextender of any $F \in \operatorname{Ult}(V, E)$.

Proof. Suppose not. Set $E_{0}=E$ and $E_{1}=F$. Let $E_{0}$ be a subextender of $E_{1}$, via $\pi$. There is a natural embedding $\sigma: \operatorname{Ult}\left(V, E_{0}\right) \rightarrow \operatorname{Ult}\left(V, E_{1}\right)$ given by

Clearly $\sigma \uparrow\left(\operatorname{crit}\left(E_{0}\right)+1\right)=$ id. Let

$$
\sigma\left([a, f]_{E_{0}}^{V}\right)=\left[\pi^{\prime \prime} a, f\right]_{E_{1}}^{V}
$$

$$
E_{2}=\sigma\left(E_{1}\right)
$$

Then $E_{1}$ is a subextender of $E_{2}$ via $\sigma$, and $E_{2} \in \operatorname{Ult}\left(V, E_{1}\right)$, so we can continue to get $E_{3}, E_{4}$, etc. The result is an infinite descending $\triangleleft$-chain of extenders with the same critical point, a contradiction.

Corollary 3.11 generalizes the easily proved fact that $E \notin \operatorname{Ult}(V, E)$. We do not know how to prove the corollary without resorting to Corollary 3.10 . Also, we do not know whether Corollary 3.11 is true if the notion of subextender is liberalized by dropping the condition $\pi(\kappa)=\kappa$.

The second author has recently proved an extension of Theorem 3.9 which implies that, on arbitrary extenders, the Mitchell order is wellfounded. This work will be published elsewhere.

Although we cannot show that every plus one iteration tree of length $\omega$ on a premouse $\mathscr{M} \prec\left(V_{\alpha}, \in, \delta\right)$ has a cofinal wellfounded branch, we can do so granted the additional hypothesis that $\mathscr{M}$ is countable. In fact, we can then prove that there are even "better" branches. (We shall use the existence of "better" branches in the theory of inner models with more than one Woodin cardinal.) The idea behind the proof is an important ingredient in $\S 4$; it was inspired by Jensen's proof [ $\mathrm{J} 2]$ that $V$ is linearly iterable. 
Theorem 3.12. Let $\mathscr{T}$ be a plus one iteration tree of length $\omega$ on a countable premouse $\mathscr{M}$. Suppose $\pi: \mathscr{M} \rightarrow\left(V_{\alpha}, \in, \delta\right)$ is elementary. Then there is a cofinal branch $b$ of $\mathscr{T}$ and an elementary $\sigma: \mathscr{M}_{b}^{\mathscr{T}} \rightarrow\left(V_{\alpha}, \in, \delta\right)$ such that the diagram

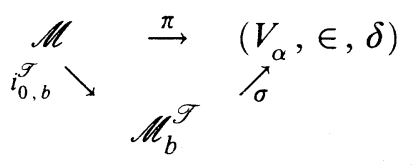

commutes.

Proof. Suppose $\mathscr{T}$ and $\pi: \mathscr{M} \rightarrow\left(V_{\alpha}, \in, \delta\right)$ constitute a counterexample to Lemma 3.2. Let $\mathscr{P}=\left(V_{\eta}, \in, \delta\right)$ be a premouse such that $\eta>\alpha$. Let $\pi \mathscr{T}$ be the copied tree on $\mathscr{P}=\mathscr{P}_{0}^{\pi \mathscr{T}}$. We shall show that $\pi \mathscr{T}$ is continuously illfounded. This contradicts Theorem 3.9 and thereby completes the proof.

Notice that $\pi, \mathscr{M} \in \mathscr{P}$ and $\mathscr{M}$ is countable in $\mathscr{P}$. Thus in $\mathscr{P}$ we can form a tree (set of finite sequences closed under initial segment) $\mathscr{U}$ whose infinite branches correspond exactly to pairs $(b, \sigma)$ such that $b$ is a cofinal branch of $\mathscr{T}$ and $\sigma: \mathscr{M}_{b}^{\mathscr{T}} \rightarrow\left(V_{\alpha}, \in, \delta\right)$ and $\pi=\sigma \circ i_{0, b}^{\mathscr{T}}$. More precisely, working in $\mathscr{P}$, let $m: \omega \longrightarrow\left(\omega \times \bigcup_{\beta<\omega} \mathscr{M}_{\beta}^{\mathscr{T}}\right)$ be such that $m^{-1}((\beta, x))$ is infinite whenever $x \in \mathscr{M}_{\beta}^{\mathscr{T}}$. We put $\left\langle\left(\beta_{i}, y_{i}\right) \mid i \leq n\right\rangle$ into $\mathscr{U}$ iff

(a) $\beta_{0}=0 \wedge \forall i<n\left(\beta_{i} \mathrm{~T} \beta_{i+1}\right)$, and

(b) $\left(\mathscr{M}_{\beta_{n}}^{\mathscr{T}}, z_{0}, \ldots, z_{n}\right) \equiv\left(V_{\alpha}, \in, \delta, y_{0}, \ldots, y_{n}\right)$, where $z_{e}=i_{\gamma \beta_{n}}^{\mathscr{T}}(x)$ if $m(e)=(\gamma, x)$ where $\gamma \in\left[0, \beta_{e}\right]_{\mathrm{T}}$ and $x \in \mathscr{M}_{\gamma}^{\mathscr{T}}$, and $z_{e}=\varnothing$ otherwise, and $y_{e}=\pi(x)$ if $m(e)=(0, x)$ where $x \in \mathscr{M}_{0}^{\mathscr{g}}$.

Notice that $\mathscr{U}$ is wellfounded, that is, has no infinite branches, by hypothesis.

Let us write $\mathscr{M}_{i}=\mathscr{M}_{i}^{\mathscr{T}}$ and $\mathscr{P}_{i}=\mathscr{M}_{i}^{\pi \mathscr{T}}$ for $i \in \omega$. Similarly, for $b$ a branch of $\mathrm{T}$ we write $\mathscr{M}_{b}=\mathscr{M}_{b}^{\mathscr{T}}$ and $\mathscr{P}_{b}=\mathscr{M}_{b}^{\pi \mathscr{T}}$ for the direct limits along $b$ in $\mathscr{T}$ and $\pi \mathscr{T}$. For any branch $b$ of $\mathrm{T}$, the copying construction gives an elementary

$$
\pi_{b}: \mathscr{M}_{b} \rightarrow\left(V_{\alpha_{b}}^{\mathscr{P}_{b}}, \in, \delta^{\mathscr{P}_{b}}\right),
$$

where $\alpha_{b}=i_{0, b}^{\pi \mathscr{T}}(\alpha) ;$ moreover, $\pi_{b} \circ i_{0, b}^{\mathscr{T}}=i_{0, b}^{\pi \mathscr{T}} \circ \pi$. But now $\operatorname{dom}(\pi)$ is hereditarily countable in $\mathscr{P}_{0}$, so $i_{0, b}^{\pi \mathscr{T}}(\pi(x))=i_{0, b}^{\pi \mathscr{T}}(\pi)\left(i_{0, b}^{\pi \mathscr{T}}(x)\right)=i_{0, b}^{\pi \mathscr{T}}(\pi)(x)$. Thus $i_{0, b}^{\pi \mathscr{T}} \circ \pi=i_{0, b}^{\pi \mathscr{T}}(\pi)$, and

$$
i_{0, b}^{\pi \mathscr{T}}(\pi)=\pi_{b} \circ i_{0, b}^{\mathscr{T}}
$$

It follows that the pair $\left(b, \pi_{b}\right)$ determines a branch of $i_{0, b}^{\pi \mathscr{T}}(\mathscr{U})$; moreover, this branch is determined continuously from $b$ so that we can take $\theta_{n}$ to be the rank in $\mathscr{P}_{n}$ of $i_{0, b}^{\pi \mathscr{T}}(\mathscr{U})$ below an appropriate initial segment of the branch.

More precisely, let $\pi_{n}: \mathscr{M}_{n} \rightarrow\left(V_{\alpha_{n}}^{\mathscr{P}_{n}}, \in, \delta^{\mathscr{P}_{n}}\right)$, where $\alpha_{n}=i_{0, n}^{\pi \mathscr{T}}(\alpha)$, be the canonical embedding from the copying construction. Fix $k \in \omega$, and let $\left\langle\beta_{i} \mid i \leq n\right\rangle$ enumerate $[0, k]_{\mathrm{T}}$ in increasing order. For any $e \leq n$, let 
$m(e)=(\gamma, x)$, and set

$$
y_{e}=i_{\gamma, \beta_{n}}^{\pi \mathscr{T}}\left(\pi_{\gamma}(x)\right)
$$

if $\gamma \in\left[0, \beta_{e}\right]_{\mathrm{T}}$, and $y_{e}=\varnothing$ otherwise. Finally, set

$$
\theta_{k}=\text { rank of } i_{0, k}^{\pi \mathscr{T}}(\mathscr{U}) \text { below }\left\langle\left(\beta_{i}, y_{i}\right) \mid i \leq n\right\rangle .
$$

One can check routinely that the $\theta_{k}$ 's witness the continuous illfoundedness of $\pi \mathscr{T}$, the desired contradiction.

The proofs of Theorems 3.7, 3.9, and 3.12 contain most of the ideas we use in $\S 4$. We now describe briefly the remaining ideas, which concern the construction of transfinite stages in an enlargement.

Definition 3.13. Let $\mathscr{T}$ be an iteration tree of length $\omega$ on $\mathscr{M}$, and let $b$ be a cofinal branch of $\mathscr{T}$. We say $\mathscr{T}$ is continuously illfounded off $b$ iff there are ordinals $\theta_{n} \in \mathscr{M}_{n}^{\mathscr{T}}$, for $n \in \omega$, such that whenever $m \notin b$ and $n \mathrm{~T} m$, $i_{n m}^{\mathscr{T}}\left(\theta_{n}\right)>\theta_{m}$, and whenever $m \in b, \theta_{m}=i_{0 m}^{\mathscr{T}}\left(\theta_{0}\right)$.

Suppose $\mathscr{M}$ is a countable premouse and $\pi: \mathscr{M} \rightarrow\left(V_{\nu}, \in, \delta\right)$ is elementary. Let $\mathscr{T}$ be a plus one iteration tree of length $\omega+1$ on $\mathscr{M}$ such that $\mathscr{T} \uparrow \omega$ is continuously illfounded off $b=[0, \omega]_{\mathrm{T}}$. We shall sketch the construction of an enlargement $\left\langle\left(\pi_{\gamma}, \mathscr{P}_{\gamma}, \nu_{\gamma}\right) \mid \gamma \leq \omega\right\rangle$ of $\mathscr{T}$ such that $\pi_{\gamma} \in \mathscr{P}_{\gamma}$ for $\gamma \in \omega$, and $\mathscr{P}_{\gamma+1} \in \mathscr{P}_{\gamma}$ whenever $\gamma+1 \notin b$. Let $\theta_{n}, n \in \omega$, witness the continuous illfoundedness of $\mathscr{T} \uparrow \omega$ off $b$. Let $\pi_{0}=\pi, \nu_{0}=\nu$, and $\mathscr{P}_{0}=\left(V_{\eta}, \in, \delta\right)$ where $\eta$ is large enough that $\left\{\gamma \mid \nu_{0}<\gamma<\eta \wedge\left(V_{\gamma}, \in, \delta\right)\right.$ is a premouse $\}$ has order type $\theta_{0}$. Now suppose we have defined $\left(\pi_{\gamma}, \mathscr{P}_{\gamma}, \nu_{\gamma}\right)$, where $\gamma<\omega$. There are two cases, depending on whether $\gamma+1 \in b$ or not. If $\gamma+1 \notin b$, then we use the argument of Theorem 3.9. Let $\xi=\mathrm{T}$-pred $(\gamma+1)$, and $\mathscr{Q}=\operatorname{Ult}\left(\mathscr{P}_{\xi}, \pi_{\gamma}\left(E_{\gamma}^{\mathscr{G}}\right)\right) . \mathscr{P}_{\gamma+1}$ is a hull of a level of $\mathscr{Q}$; we use $\theta_{\gamma+1}$ to tell us which level, or, intuitively, how much ground to give. The hull is small enough that $\mathscr{P}_{\gamma+1} \in \mathscr{P}_{\gamma}$. We get $\pi_{\gamma+1} \in \mathscr{P}_{\gamma+1}$ by an absoluteness argument. There is just one wrinkle here: we cannot use the argument from Theorem 3.9 that $\mathscr{Q}$ is wellfounded, as $\mathscr{Q}$ may not be an internal ultrapower of $\mathscr{P}_{\xi}$ if $\exists \eta \in b(\xi<\eta \leq \gamma)$. So instead we use the proof of Theorem 3.7; the $\mathscr{P}$ 's are close enough to being the models of an iteration tree that this argument goes through.

If $\gamma+1 \in b$, we simply set $\mathscr{P}_{\gamma+1}=\operatorname{Ult}\left(\mathscr{P}_{\xi}, \pi_{\gamma}\left(E_{\gamma}^{\mathscr{G}}\right)\right)$, where $\xi=$ T-pred $(\gamma+1)$. Thus we have a canonical embedding $j_{\xi, \gamma+1}: \mathscr{P}_{\xi} \rightarrow \mathscr{P}_{\gamma+1}$. We get $\pi_{\gamma+1} \in \mathscr{P}_{\gamma+1}$ again by an absoluteness argument. Notice that $\mathscr{P}_{\gamma+1}$ is an internal ultrapower of $\mathscr{P}_{\xi}$ since $\mathscr{P}_{\eta+1} \in \mathscr{P}_{\eta}$ for $\eta+1 \notin b$.

Now let $\mathscr{P}_{\omega}=$ direct limit of $\mathscr{P}_{\xi}, \xi \in b$, under the $j_{\xi, \gamma}$ 's. $\mathscr{P}_{\omega}$ is wellfounded as the ultrapowers along $b$ at the enlargement level are internal. We would like to define $\pi_{\omega}: \mathscr{M}_{\omega}^{\mathscr{T}} \rightarrow\left(V_{\nu_{\omega}}^{\mathscr{P}_{\omega}}, \in, \delta^{\mathscr{P}_{\omega}}\right)$, where $\nu_{\omega}=j_{0, \omega}\left(\nu_{0}\right)$, by $\pi_{\omega}\left(i_{\xi \omega}^{\mathscr{G}}(x)\right)=j_{\xi \omega}\left(\pi_{\xi}(x)\right)$. The trouble is that if $\xi=\mathrm{T}$-pred $(\gamma+1), \gamma+1 \in b$, then $\pi_{\gamma+1} \circ i_{\xi, \gamma+1}^{\mathscr{G}}=j_{\xi, \gamma+1} \circ \pi_{\xi}$ may fail. But we do have $\pi_{\gamma+1} \circ i_{\xi, \gamma+1}^{\mathscr{g}}=$ 
$j_{\xi, \gamma+1} \circ \tau$, where $\tau$ is the natural embedding from the shift lemma, so for any finite $X \subseteq \mathscr{M}_{\xi}^{\mathscr{T}}$ we can arrange in the absoluteness argument producing $\pi_{\gamma+1}$ that $\left(\pi_{\gamma+1} \circ i_{\xi, \gamma+1}^{\mathscr{T}}\right)(x)=\left(j_{\xi, \gamma+1} \circ \pi_{\xi}\right)(x)$ for all $x \in X$. Suppose we choose $X=X_{\xi} \subseteq \mathscr{M}_{\xi}^{\mathscr{T}}$ so that for all $\xi \in b$ and $x \in \mathscr{M}_{\xi}^{\mathscr{T}}, i_{\xi, \eta}^{\mathscr{T}}(x) \in X_{\eta}$ for all sufficiently large $\eta \in b$. We can then define $\pi_{\omega}$ by: $\pi_{\omega}\left(i_{\xi, \omega}^{\mathscr{T}}(x)\right)=j_{\xi, \omega}\left(\pi_{\xi}(x)\right)$ for $\xi \in b$ and $x$ such that $i_{\xi, \eta}^{\mathscr{T}}(x) \in X_{\eta}$ for all $\eta \in b \backslash \xi$.

To summarize: along wellfounded branches $b$ of $\mathscr{T}$ we internalize at the enlargement level and use pointwise eventual commutativity to define an embedding from $\mathscr{M}_{b}^{\mathscr{T}}$ into $\mathscr{P}_{b}$.

If we try now to arrange that $\pi_{\omega} \in \mathscr{P}_{\omega}$, we encounter our last problem. There may not be enough information in $\mathscr{P}_{\omega}$ about $\left\langle\pi_{\xi} \mid \xi<\omega\right\rangle$ to form in $\mathscr{P}_{\omega}$ a tree whose branches are embeddings which agree as required with the $\pi_{\xi}, \xi<\omega$. Our solution is to form a tree in $\mathscr{P}_{\omega}$ which attempts to build not just $\pi_{\omega}$ but new versions of the $\pi_{\xi}$ and $\mathscr{P}_{\xi}$ for $\xi<\omega$. (Thus for $\mathscr{T}$ of transfinite length, we shall build not just a single enlargement but a sequence of enlargements.) In order to show that this tree in $\mathscr{P}_{\omega}$ has a branch, we need to know that sufficiently large fragments of the old $\pi_{\xi}$ and $\mathscr{P}_{\xi}$, for $\xi<\omega$, are in $\mathscr{P}_{\omega}$. For that we seem to need that $\mathscr{T}$ is a plus two iteration tree.

This finishes our catalogue of ideas from $\S 4$. What is left is just bookkeeping.

\section{ITERABILITY}

We shall prove a general result on the extendibility of countable iteration trees on countable elementary submodels of $V$.

Definition 4.1. Let $\mathscr{T}$ be an iteration tree on $\mathscr{M}$, and $\pi: \mathscr{M} \rightarrow\left(V_{\alpha}, \in, \delta\right)$. Let $b$ be a branch of $\mathscr{T}$. We say that $b\left(\right.$ or $\left.\mathscr{M}_{b}^{\mathscr{T}}\right)$ is $\pi$-realizable if there is an elementary $\sigma: \mathscr{M}_{b}^{\mathscr{T}} \rightarrow\left(V_{\alpha}, \in, \delta\right)$ such that $\pi=\sigma \circ i_{0 b}^{\mathscr{T}}$.

Of course, being $\pi$-realizable is a property of $\mathscr{T}, \alpha$, and $\delta$, as well as of $b$, but these other parameters will be determined from context.

Definition 4.2. Let $\mathscr{T}$ be an iteration tree on $\mathscr{M}, \pi: \mathscr{M} \rightarrow\left(V_{\gamma}, \in, \delta\right)$ and $\alpha<\beta<\operatorname{lh}(\mathscr{T})$. Suppose $\mathscr{M}_{\beta}^{\mathscr{T}} \models$ “ $E$ is an extender", and suppose $\operatorname{crit}(E)<$ $\rho^{\mathscr{T}}(\alpha, \beta)$ so that $\operatorname{Ult}\left(\mathscr{M}_{\alpha}^{\mathscr{T}}, E\right)$ makes sense. Let $\tau: \mathscr{M}_{\alpha}^{\mathscr{T}} \rightarrow \operatorname{Ult}\left(\mathscr{M}_{\alpha}^{\mathscr{T}}, E\right)$ be the canonical embeddding. We say that $\operatorname{Ult}\left(\mathscr{M}_{\alpha}^{\mathscr{T}}, E\right)$ is $\pi$-realizable iff there is an elementary

$$
\sigma: \operatorname{Ult}\left(\mathscr{M}_{\alpha}^{\mathscr{T}}, E\right) \rightarrow\left(V_{\gamma}, \in, \delta\right)
$$

such that $\pi=\sigma \circ\left(\tau \circ i_{0 \alpha}^{\mathscr{T}}\right)$.

Again, certain parameters are determined by context.

The following observations may clarify this concept. Let $\mathscr{T}$ be a countable iteration tree on a countable $\mathscr{M}$, and $\pi: \mathscr{M} \rightarrow\left(V_{\alpha}, \in, \delta\right)$. Let $\mathscr{P}=\left(V_{\eta}\right.$, $\in, \delta$ ) be a premouse with $\alpha<\eta$. We can form the copied tree $\pi \mathscr{T}$ on $\mathscr{P}$ 
as long as the models we encounter are wellfounded; suppose this is always the case, so that $\ln (\mathscr{T})=\ln (\pi \mathscr{T})$. Let $b$ be a branch of $\mathscr{T}$, and suppose that $\mathscr{P}_{b}=\mathscr{M}_{b}^{\pi \mathscr{T}}$ is wellfounded. Then $b$ is $\pi$-realizable. [Proof: the copying construction yields an elementary $\pi_{b}: \mathscr{M}_{b}^{\mathscr{T}} \rightarrow\left(V_{\alpha_{b}}^{\mathscr{P}_{b}}, \in, \delta^{\mathscr{P}_{b}}\right)$, where $\alpha_{b}=$ $i_{0 b}^{\pi \mathscr{T}}(\alpha)$; moreover, $\pi_{b} \circ i_{0 b}^{\mathscr{T}}=i_{0 b}^{\pi \mathscr{T}} \circ \pi$. As $\operatorname{dom}(\pi)$ is hereditarily countable in $\mathscr{P}, i_{0 b}^{\pi \mathscr{T}} \circ \pi=i_{0 b}^{\pi \mathscr{T}}(\pi)$. By absoluteness there is $\tau: M_{b}^{\mathscr{T}} \rightarrow\left(V_{\alpha_{b}}^{\mathscr{P}_{b}}, \in, \delta^{\mathscr{P}_{b}}\right)$ such that $\tau \circ i_{0 b}^{\mathscr{T}}=i_{0 b}^{\pi \mathscr{T}}(\pi)$ and $\tau \in \mathscr{P}_{b}$. That is, $\mathscr{P}_{b} \models$ " $b$ is $i_{0 b}^{\pi \mathscr{T}}(\pi)$-realizable". The elementarity of $i_{0 b}^{\pi \mathscr{T}}$ implies that $b$ is $\pi$-realizable.] Thus the $\pi$-realizability of $b$ implies the wellfoundedness of $\mathscr{M}_{b}^{\mathscr{T}}$ and is implied by the wellfoundedness of $\mathscr{M}_{b}^{\pi \mathscr{T}}$. Similar remarks apply to the $\pi$-realizability of Ult $\left(\mathscr{M}_{\alpha}^{\mathscr{T}}, E\right)$.

Our main theorem on iterability is

Theorem 4.3. Let $\mathscr{T}$ be a plus two iteration tree of countable length on a countable premouse $\mathscr{M}$, and let $\pi: \mathscr{M} \rightarrow\left(V_{\alpha}, \in, \delta\right)$ be elementary. Suppose there is no maximal branch $b$ of $\mathscr{T}$ such that $\sup (b)<\ln (\mathscr{T})$ and $b$ is $\pi$-realizable. Then

(a) if $\operatorname{lh}(\mathscr{T})$ is a limit ordinal, then $\mathscr{T}$ has a cofinal branch which is $\pi$ realizable; and

(b) if $\beta<\gamma<\operatorname{lh}(\mathscr{T}), \mathscr{M}_{\gamma}^{\mathscr{T}} \models$ " $E$ is an extender", and $\operatorname{crit}(E)+1<$ $\rho^{\mathscr{T}}(\beta, \gamma)$, then $\operatorname{Ult}\left(\mathscr{M}_{\beta}^{\mathscr{T}}, E\right)$ is $\pi$-realizable.

We shall derive Theorem 4.3 from a weaker version of itself involving continuously illfounded trees, just as we derived Theorem 3.12 from Theorem 3.9.

Definition 4.4. Let $\mathscr{T}$ be an iteration tree of countable limit length $\lambda$ on a premouse $\mathscr{M}$. Let $X$ be a cofinal subset of $\lambda$ of order type $\omega$. Then

(a) $(\mathscr{T}, M)$ is continuously illfounded iff there are ordinals $\theta_{\alpha} \in \mathscr{M}_{\alpha}^{\mathscr{T}}$, for $\alpha<\lambda$, such that whenever $\alpha \mathrm{T} \beta, i_{\alpha \beta}^{\mathscr{T}}\left(\theta_{\alpha}\right)>\theta_{\beta}$ if $\exists \gamma \in X(\alpha<\gamma \leq \beta)$ and $i_{\alpha \beta}^{\mathscr{T}}\left(\theta_{\alpha}\right)=\theta_{\beta}$ otherwise;

(b) if $b$ is a cofinal branch of $\mathscr{T}$, then $(\mathscr{T}, M)$ is continuously illfounded off $b$ if there are ordinals $\theta_{\alpha} \in \mathscr{M}_{\alpha}^{\mathscr{T}}$, for $\alpha<\lambda$, such that whenever $\alpha \mathrm{T} \beta$, $i_{\alpha \beta}^{\mathscr{T}}\left(\theta_{\alpha}\right)>\theta_{\beta}$ if $\beta \notin b$ and $\exists \gamma \in X(\alpha<\gamma \leq \beta)$, and $i_{\alpha \beta}^{\mathscr{T}}\left(\theta_{\alpha}\right)=\theta_{\beta}$ otherwise.

Notice that we need not say "continuously illfounded with respect to $X$ ", since if $X$ and $Y$ are cofinal subsets of $\lambda$ of order type $\omega$, then $(\mathscr{T}, M)$ is continuously illfounded with respect to $X$ iff $(\mathscr{T}, M)$ is continuously illfounded with respect to $Y$. We sketch the proof of this in the case $X \subseteq Y$ and leave the rest to the reader. Let $\left\langle\theta_{\alpha} \mid \alpha<\lambda\right\rangle$ witness that $(\mathscr{T}, M)$ is continuously illfounded with respect to $X$, and for $\alpha<\lambda$ let $n(\alpha)$ be the (finite) number of elements of $Y$ between $\alpha$ and $\inf (X \backslash(\alpha+1))$; then the ordinals $\eta_{\alpha}=\omega \cdot \theta_{\alpha}+n(\alpha)$ witness that $(\mathscr{T}, M)$ is continuously illfounded "with respect to $Y$ ".

Definition 4.5. Let $\mathscr{T}$ be an iteration tree of countable length on a premouse $\mathscr{M}$. Then $\mathscr{T}, M$ is self-justifying iff for every limit $\lambda<\ln (\mathscr{T}),(\mathscr{T} \uparrow \lambda, \mathscr{M})$ is continuously illfounded off $[0, \lambda)_{\mathrm{T}}$. 
Lemma 4.6. Let $\mathscr{T}$ be a plus two iteration tree of countable length on a premouse $\mathscr{M}$ which is elementarily embeddable into some $\left(V_{\alpha}, \in, \delta\right)$. Suppose $(\mathscr{T}, M)$ is self-justifying. Then

(a) $\mathscr{T}$ is not continuously illfounded, and

(b) if $\beta<\gamma<\ln (\mathscr{T}), \mathscr{M}_{\gamma}^{\mathscr{T}} \models$ " $E$ is an extender," and $\operatorname{crit}(E)+1<$ $\rho^{\mathscr{T}}(\beta, \gamma)$, then $\operatorname{Ult}\left(\mathscr{M}_{\beta}^{\mathscr{T}}, E\right)$ is wellfounded.

Let us assume Lemma 4.6 for the moment, and finish the proof of Theorem 4.3 .

Proof of Theorem 4.3. Let $\mathscr{T}$ and $\pi: \mathscr{M} \rightarrow\left(V_{\alpha}, \in, \delta\right)$ furnish a counterexample. Let $\eta>\alpha$ be such that $\mathscr{P}=\left(V_{\eta}, \in, \delta\right)$ is a premouse. Let $\pi \mathscr{T}$ be the copied tree on $\mathscr{P}$. (We are not assuming $\operatorname{lh}(\pi \mathscr{T})=\operatorname{lh}(\mathscr{T}) ; \pi \mathscr{T}$ may encounter an illfounded model at some stage $<\operatorname{lh}(\mathscr{T})$ and, at that stage, become undefined.)

Claim 1. $(\pi \mathscr{T}, \mathscr{P})$ is self-justifying.

Proof. Let $\lambda<\operatorname{lh}(\pi \mathscr{T})$ by a limit ordinal, and let $b=[0, \lambda)_{\mathrm{T}}$ be the cofinalin- $\lambda$ branch of $\pi \mathscr{T}$. Let $X \subseteq \lambda$ be cofinal and have order type $\omega$. Using $X$ we can define a tree $\mathscr{U} \in \mathscr{P}$ whose infinite branches are precisely those $(c, \sigma)$ such that $c$ is a cofinal in $\lambda$ branch of $\mathscr{T}, c \neq b$, and $\sigma: \mathscr{M}_{c}^{\mathscr{T}} \rightarrow\left(V_{\alpha}, \in, \delta\right)$ and $\pi=\sigma \circ i_{0 c}^{\mathscr{g}}$. The tree $\mathscr{U}$ is wellfounded by hypothesis, but $i_{0 c}^{\pi \mathscr{T}}(\mathscr{U})$ has a branch whenever $c$ is a cofinal-in- $\lambda$ branch of $\mathscr{T}$ and $c \neq b$; namely $\left(c, \pi_{c}\right)$, where $\pi_{c}$ comes from the copying construction. Thus $\mathscr{P}_{c}$ is illfounded, and one can check that the illfoundedness is given continuously.

More precisely, we define $\mathscr{U}$ as follows. Fix a map $m: \omega \rightarrow\left(\lambda \times \bigcup_{\beta<\lambda} \mathscr{M}_{\beta}^{\mathscr{T}}\right)$ such that $m^{-1}((\beta, x))$ is infinite whenever $\beta<\lambda$ and $x \in \mathscr{M}_{\beta}^{\mathscr{T}}$. We put a pair $\left(\left\langle\alpha_{0} \cdots \alpha_{n}\right\rangle,\left\langle y_{0} \cdots y_{n}\right\rangle\right)$ of sequences into $\mathscr{U}$ iff

(1) $\alpha_{0} \notin b \wedge \forall i<n\left(\alpha_{i} \mathrm{~T} \alpha_{i+1} \wedge \exists \gamma \in X\left(\alpha_{i}<\gamma \leq \alpha_{i+1}\right)\right)$, and

(2) $\left(\mathscr{M}_{\alpha_{n}}^{\mathscr{T}}, z_{0} \cdots z_{n}\right) \equiv\left(V_{\alpha}, \in, \delta, y_{0} \cdots y_{n}\right)$, where for $e \leq n, z_{e}=$ $i_{\beta, \alpha_{n}}^{\mathscr{G}}(x)$ if $m(e)=(\beta, x)$ and $\beta \mathrm{T} \alpha_{e}$ and $x \in \mathscr{M}_{\beta}^{\mathscr{T}}$, and $z_{e}=\varnothing$ otherwise, and for $e \leq n, y_{e}=\pi(x)$ if $m(e)=(0, x)$ with $x \in \mathscr{M}_{0}^{\mathscr{T}}$.

Clearly, $\mathscr{U}$ is wellfounded. Now for $\gamma<\lambda$, we let $\theta_{\gamma}=\operatorname{rank}$ of $(\varnothing, \varnothing)$ in $i_{0 \gamma}^{\pi \mathscr{T}}(\mathscr{U})$ if $\gamma \in b$ and

$$
\theta_{\gamma}=\operatorname{rank} \text { of }\left(\left\langle\alpha_{0} \cdots \alpha_{n}\right\rangle,\left\langle y_{0} \cdots y_{n}\right\rangle\right) \text { in } i_{0 \gamma}^{\pi \mathscr{S}}(\mathscr{U})
$$

if $\gamma \notin b$, where

$$
\begin{aligned}
\alpha_{0} & =\text { least in }[0, \gamma]_{\mathrm{T}} \backslash b, \\
\alpha_{i+1} & =\text { least } \eta \in[0, \gamma]_{\mathrm{T}} \text { such that } \exists \xi \in X\left(\alpha_{i}<\xi \leq \alpha_{i+1}\right),
\end{aligned}
$$

and $n$ is largest such that $\alpha_{n}$ is defined, and for $e \leq n, y_{e}=\pi_{\alpha_{n}}\left(z_{e}\right)$, where $z_{e}=i_{\beta, \alpha_{n}}^{\mathscr{G}}(x)$ if $m(e)=(\beta, x)$ with $\beta \mathrm{T} \alpha_{e}$ and $x \in \mathscr{M}_{\beta}^{\mathscr{J}^{n}}$, and 
$z_{e}=\varnothing$ otherwise. Here $\pi_{\alpha_{n}}: \mathscr{M}_{\alpha_{n}}^{\mathscr{T}} \rightarrow i_{0, \alpha_{n}}^{\pi \mathscr{T}}\left(\left(V_{\alpha}, \in, \delta\right)\right)$ comes from the copying construction. One can check routinely that $\left\langle\theta_{\gamma} \mid \gamma<\lambda\right\rangle$ witnesses that $(\pi \mathscr{T} \uparrow \lambda, \mathscr{P})$ is continuously illfounded off $b$.

Claim 2. $\ln (\pi \mathscr{T})=\operatorname{lh}(\mathscr{T})$.

Proof. As $\pi \mathscr{T}$ is self-justifying, Lemma 4.6 implies that we do not encounter illfoundedness at successor steps. Suppose then that $\lambda=\ln (\pi \mathscr{T})$ is a limit ordinal and $\lambda<\operatorname{lh}(\mathscr{T})$. Let $b=[0, \lambda)_{\mathrm{T}}$ so that $\mathscr{M}_{b}^{\pi \mathscr{T}}$ is illfounded. The proof of Claim 1 shows that $\pi \mathscr{T}$ is continuously illfounded off $b$. But since $\mathscr{M}_{b}^{\pi \mathscr{T}}$ is itself illfounded, this means that $\pi \mathscr{T}$ is continuously illfounded. As $\pi \mathscr{T}$ is self-justifying, this contradicts Lemma 4.6.

Now let us consider the two ways $\mathscr{T}$ might fail to satisfy the conclusion of Theorem 4.3. Suppose first $\ln (\mathscr{T})=\lambda$ is a limit ordinal and no cofinal-in- $\lambda$ branch of $\mathscr{T}$ is $\pi$-realizable. The proof of Claim 1 then shows that $\pi \mathscr{T}$ is continuously illfounded, contradicting Lemma 4.6. So suppose $\beta<\gamma<\operatorname{lh}(\mathscr{T})$, $\mathscr{M}_{\gamma}^{\mathscr{T}} \models$ “E is an extender", $\operatorname{crit}(E)+1<\rho^{\mathscr{T}}(\beta, \gamma)$, and $\operatorname{Ult}\left(\mathscr{M}_{\beta}^{\mathscr{T}}, E\right)$ is not $\pi$-realizable. For any $\eta<\operatorname{lh}(\mathscr{T})$, let $\pi_{\eta}: \mathscr{M}_{\eta}^{\mathscr{T}} \rightarrow i_{0 \eta}^{\pi \mathscr{T}}\left(\left(V_{\alpha}, \in, \delta\right)\right)$ be given by the copying construction. Now $\operatorname{Ult}\left(\mathscr{M}_{\beta}^{\pi \mathscr{T}}, \pi_{\gamma}(E)\right)$ is wellfounded by Lemma 4.6 and the fact $\pi \mathscr{T}$ is self-justifying. Let $\tau: \mathscr{M}_{\beta}^{\mathscr{T}} \rightarrow \operatorname{Ult}\left(\mathscr{M}_{\beta}^{\mathscr{T}}, E\right)$ and $\psi: \mathscr{M}_{\beta}^{\pi \mathscr{T}} \rightarrow \operatorname{Ult}\left(\mathscr{M}_{\beta}^{\pi \mathscr{T}}, \pi_{\gamma}(E)\right)$ be the canonical embedings, and let

$$
\sigma: \operatorname{Ult}\left(\mathscr{M}_{\beta}, E\right) \rightarrow \psi \circ i_{0 \beta}^{\pi \mathscr{T}}\left(\left(V_{\alpha}, \in, \delta\right)\right)
$$

be given by the shift lemma. We have that

$$
\sigma \circ\left(\tau \circ i_{0 \beta}^{\mathscr{T}}\right)=\left(\psi \circ i_{0 \beta}^{\pi \mathscr{T}}\right) \circ \pi=\left(\psi \circ i_{0 \beta}^{\pi \mathscr{T}}\right)(\pi) .
$$

By absoluteness, there is a $\sigma^{*} \in \operatorname{Ult}\left(\mathscr{M}_{\beta}^{\pi \mathscr{T}}, \pi_{\gamma}(E)\right)$ such that

$$
\sigma^{*}: \operatorname{Ult}\left(\mathscr{M}_{\beta}^{\mathscr{T}}, E\right) \rightarrow \psi \circ i_{0 \beta}^{\pi \mathscr{T}}\left(\left(V_{\alpha}, \in, \delta\right)\right)
$$

and $\sigma^{*} \circ\left(\tau \circ i_{0 \beta}^{\mathscr{T}}\right)=\left(\psi \circ i_{0 \beta}^{\pi \mathscr{T}}\right)(\pi)$. That is, $\operatorname{Ult}\left(\mathscr{M}_{\beta}^{\mathscr{I}}, E\right)$ is $\left(\psi \circ i_{0 \beta}^{\pi \mathscr{T}}\right)(\pi)$ realizable in the model $\operatorname{Ult}\left(\mathscr{M}_{\beta}^{\pi \mathscr{T}}, \pi_{\gamma}(E)\right)$. This implies that $\operatorname{Ult}\left(\mathscr{M}_{\beta}^{\mathscr{T}}, E\right)$ is $\pi$-realizable in $V$. This contradiction finishes the proof of Theorem 4.3.

The rest of this section is devoted to the proof of Lemma 4.6.

The heart of the proof is the construction of a certain sequence of enlargements of a putative bad $\mathscr{T}$. We make use of the following bookkeeping device in this construction.

Definition 4.7. Let $\mathrm{T}$ be a tree order on $\lambda$, and let $p: \lambda \rightarrow \lambda$. We call $p$ a preservation function for $\mathrm{T}$ iff

(1) $p(\alpha) \leq \alpha$ for all $\alpha<\lambda, p(\gamma)<\gamma$ for all limit $\gamma<\lambda$, and $p(\alpha)$ is a successor ordinal for all $\alpha<\lambda, \alpha \neq 0$;

(2) for limit $\gamma \leq \lambda, \lim \inf _{\alpha \rightarrow \gamma} p(\alpha)=\gamma$; and 
(3) if $\gamma<\lambda$ is a limit ordinal, then for all sufficiently large $\alpha<\gamma$ such that $\alpha \mathrm{T} \gamma$

(a) $(\alpha<\eta<\gamma \wedge \neg \eta \mathrm{T} \gamma) \Rightarrow p(\eta)>\alpha$, and

(b) if $\delta \mathrm{T} \gamma$ and $\alpha=\mathrm{T}$-pred $(\delta)$, then $p(\delta)=\alpha+1$.

Definition 4.8. Let $p$ be a preservation function for $\mathrm{T}$. We say that $\alpha$ survives at $\beta$ (relative to $p$ ) iff $\alpha \mathrm{T} \beta$ and

(1) $\forall \gamma \in[\alpha, \beta)_{\mathrm{T}} \forall \eta[(\gamma<\eta<\beta \wedge \neg \eta \mathrm{T} \beta) \Rightarrow p(\eta)>\gamma]$, and

(2) $\forall \delta \in(\alpha, \beta]_{\mathrm{T}} \forall \gamma(\gamma=\mathrm{T}$-pred $(\delta) \Rightarrow p(\delta)=\gamma+1)$.

Let $\alpha T \gamma T \beta$; then it is easy to see that $\alpha$ survives at $\beta$ if and only if $\alpha$ survives at $\gamma$ and $\gamma$ survives at $\beta$. Notice also that condition (3) in the definition of "preservation function" just asserts that all sufficiently large $\alpha \mathrm{T} \gamma$ survive at $\gamma$. Finally, notice that it is possible that $\alpha$ survives at $\beta$ and yet for some $\gamma$ such that $\alpha<\gamma<\beta, p(\gamma) \leq \alpha$. In this case, $\gamma$ must be a limit ordinal such that $\alpha \mathrm{T} \gamma \mathrm{T} \beta$.

Lemma 4.9. Let $\mathrm{T}$ be a tree order on $\lambda$, where $\lambda$ is countable. There is a preservation function for $\mathrm{T}$.

Proof. Fix $n: \lambda \stackrel{1-1}{\rightarrow} \omega$, and for $\alpha<\lambda$ let

$$
\operatorname{bi}(\alpha)=\left\{\begin{array}{l}
\text { the least } k \text { such that } k=n(\gamma) \text { for some limit } \gamma \text { with } \alpha \mathrm{T} \gamma \\
\text { if such a } k \text { exists } \\
\omega \quad \text { if no such } k \text { exists }
\end{array}\right.
$$

("bi" is for "branch index"). We first define an approximation $q$ to the desired $p$. Let $\alpha<\lambda$.

Case 0. $\alpha=0$. Set

$$
q(\alpha)=0
$$

Case 1. $\exists \beta(\beta=\mathrm{T}-\operatorname{pred}(\alpha))$. We set

$$
q(\alpha)= \begin{cases}\beta+1 & \text { if } \forall \eta(\beta<\eta<\alpha \Rightarrow \operatorname{bi}(\eta) \geq \operatorname{bi}(\alpha)), \\ \alpha & \text { otherwise }\end{cases}
$$

where $\beta=\mathrm{T}$-pred $(\alpha)$.

Case 2. $\alpha$ is a T-limit. In this case, let

$$
\begin{aligned}
F & =\{\eta<\alpha \mid n(\eta)<n(\alpha)\} \\
G & =\{\eta<\alpha \mid \neg \eta \mathrm{T} \alpha \wedge \mathrm{bi}(\eta) \leq n(\alpha)\} \\
H & =\{\eta<\alpha \mid \eta \mathrm{T} \alpha \wedge \mathrm{bi}(\eta) \neq n(\alpha) \wedge \operatorname{bi}(\eta) \neq \operatorname{bi}(\alpha)\}
\end{aligned}
$$

We set

$$
q(\alpha)=\sup (F \cup G \cup H)+1
$$

This completes the definition of $q$. We claim that if $\alpha<\lambda$ is a limit, then $q(\alpha)<\alpha$. That is, the sets $F, G$, and $H$ occurring in the definition are bounded in $\alpha$. For $F$ this is obvious as $n$ is $1-1$. If $G$ were cofinal in $\alpha$, we could fix $k \leq n(\alpha)$ such that $G^{\prime}=\{\eta \in G \mid \operatorname{bi}(\eta)=k\}$ is cofinal in $\alpha$. Let $k=n(\gamma)$, so that $G^{\prime} \subseteq[0, \gamma]_{\mathrm{T}}$. Now $[0, \gamma]_{\mathrm{T}}$ is closed in the order topology on the ordinals, so $\alpha \in[0, \gamma]_{\mathrm{T}}$. But then $\eta \in G^{\prime} \Rightarrow \eta \mathrm{T} \alpha$, 
a contradiction. Finally suppose $H$ were cofinal in $\alpha$. Now if $\eta \in H$, then bi $(\eta)<n(\alpha)$, so we can fix $k<n(\alpha)$ such that $\operatorname{bi}(\eta)=k$ for cofinally many $\eta \in H$. Let $k=n(\gamma)$. As before, $\alpha \in[0, \gamma]_{\mathrm{T}}$ as $[0, \gamma]_{\mathrm{T}}$ is closed. If $\alpha \mathrm{T} \gamma$, then $n(\gamma) \geq \operatorname{bi}(\alpha) \geq \operatorname{bi}(\eta)$ for $\eta \mathrm{T} \alpha$, so $\operatorname{bi}(\alpha)=k$, contrary to the definition of $H$. If $\alpha=\gamma$, then $n(\alpha)=k$, contrary to the fact $k=\operatorname{bi}(\eta)$ for some $\eta \in H$. Thus $F, G$, and $H$ are bounded in $\alpha$, so that $q(\alpha)<\alpha$.

We now show that $q$ has all the properties required of a preservation function, except that lim $\inf _{\alpha \rightarrow \lambda} q(\alpha)=\lambda$ may fail if $\lambda$ is a limit. Referring to Definition 4.7, we see at once that the properties in group (1) hold of $q$. Consider now (2) for limit $\gamma<\lambda$; suppose $\liminf _{\alpha \rightarrow \gamma} q(\alpha) \neq \gamma$, and let $\beta<\gamma$ be such that $q(\alpha)<\beta$ for cofinally many $\alpha<\gamma$. Let $\delta$ be such that $\delta \mathrm{T} \gamma$ and $\beta<\delta$; then $\operatorname{bi}(\delta)<\omega$ and, whenever $\alpha>\delta, q(\alpha)<\beta$, and $q(\alpha)$ is defined by case 1 , then bi $(\alpha) \leq$ bi $(\delta)$. So if $q(\alpha)$ is defined by Case 1 for cofinal in $\gamma$ many $\alpha$ such that $q(\alpha)<\beta$, we can fix bi $(\alpha)=k<\omega$ for cofinal in $\gamma$ many such $\alpha$. But then $\operatorname{bi}(\alpha)=k \Rightarrow \alpha \mathrm{T} \gamma$, as $\operatorname{bi}(\alpha)=k$ cofinally in $\gamma$. So we have $\beta<\alpha<\alpha^{\prime}<\gamma$ such that $\alpha \mathrm{T} \alpha^{\prime} \mathrm{T} \gamma$ and $q\left(\alpha^{\prime}\right)<\beta$ and $q\left(\alpha^{\prime}\right)$ is defined by Case 1 , a contradiction. Thus $q(\alpha)$ is defined by Case 2 for cofinally many $\alpha<\gamma$ such that $q(\alpha)<\beta$. But the $F$ of Case 2 is a subset of $\beta$ for such $\alpha$, so $n(\beta) \geq n(\alpha)$ for such $\alpha$, so there are finitely many such $\alpha$, a contradiction.

We now check (3). Let $\gamma<\lambda$ be a limit. Let $\beta<\gamma$ and $k<\omega$ be such that whenever $\alpha \mathrm{T} \gamma$ and $\beta \leq \alpha$, bi $(\alpha)=k$. (Here $k=n(\gamma)$ or $k=$ bi $(\gamma)$.) We also choose $\beta$ large enough that $\forall \eta(\beta \leq \eta<\gamma \Rightarrow n(\eta)>k)$ and $\forall \eta(\beta \leq \eta<\gamma \wedge \neg \eta \mathrm{T} \gamma \Rightarrow \operatorname{bi}(\eta)>k)$. We claim that (3)(a),(b) hold at all $\alpha \mathrm{T} \gamma$ such that $\beta \leq \alpha$. So fix such on $\alpha$.

For (a), let $\alpha<\eta<\gamma$ and $\neg \eta \mathrm{T} \gamma$. Suppose $q(\eta) \leq \alpha$ toward contradiction. If $q(\eta)$ is defined by Case 1 , then $k=(\alpha) \geq \operatorname{bi}(\eta)>k$. So $q(\eta)$ is defined by Case 2, and the $G$ of Case 2 is a subset of $\alpha$, so that $\alpha \notin G$. But $\alpha<\eta$ and $\operatorname{bi}(\alpha)=k<n(\eta)$, so if $\neg \alpha \mathrm{T} \eta$ then $\alpha \in G$, a contradiction. Thus $\alpha \mathrm{T} \eta$. Since $\eta>\beta$ and $\neg \eta \mathrm{T} \gamma, n(\eta)>k$ and $\operatorname{bi}(\eta)>k$. It follows that $\alpha \in H$, where $H$ is the set occurring in the definition of $q(\eta)$. Thus $q(\eta)>\alpha$, a contradiction.

For (b), if $\delta \mathrm{T} \gamma$ and $\mathrm{T}-\operatorname{pred}(\delta)=\alpha$, then $\alpha<\eta<\delta \Rightarrow \neg \eta \mathrm{T} \gamma$, so $\alpha<\eta<\delta \Rightarrow \operatorname{bi}(\eta)>k=\operatorname{bi}(\delta)$, so $q(\delta)=\alpha+1$ by Case 1 of the definition.

Thus if $\lambda$ is not a limit ordinal, then $q$ is the desired preservation function. If $\lambda$ is a limit ordinal, we must deal with the case $\gamma=\lambda$ of condition (2), and for that modify $q$. Let $\left\langle\alpha_{i} \mid i \in \omega\right\rangle$ be a strictly increasing sequence with $\sup _{i} \alpha_{i}=\lambda$. Define

$$
p(\beta)=\left\{\begin{array}{lll}
q(\beta) & \text { if } & \beta \leq \alpha_{0}+1, \\
\alpha_{i}+1 & \text { if } & \beta=\alpha_{i+1}+1, \\
\max \left(q(\beta), \alpha_{i}+2\right) & \text { if } & \alpha_{i}+1<\beta \leq \alpha_{i+1} .
\end{array}\right.
$$

If $\gamma<\lambda$ is a limit ordinal, then since $\lim \inf _{\alpha \rightarrow \gamma} q(\alpha)=\gamma$, we have that $p(\alpha)=q(\alpha)$ for all sufficiently large $\alpha<\gamma$. Using this observation, it is easy to see that $p$ is a preservation function for $\mathrm{T}$.

We shall use a preservation function to tell us, among other things, when to take hulls in constructing our sequence of enlargements. For this we want the 
ordinals witnessing continuous illfoundedness, which provide $V_{\alpha}$ 's in which to take hulls, to appear when $p$ needs them.

Definition 4.10. Let $\mathscr{T}$ be an iteration tree on $\mathscr{M}$ and $p$ a preservation function for the associated tree order $\mathrm{T}$. We say that $(\mathscr{T}, M)$ is $p$-continuously illfounded iff there are ordinals $\theta_{\alpha} \in \mathscr{M}_{\alpha}^{\mathscr{T}}$, for $\alpha<\operatorname{lh}(\mathscr{T})$, such that whenever $\alpha \mathrm{T} \beta$,

$$
\alpha \text { survives at } \beta \Rightarrow i_{\alpha \beta}^{\mathscr{T}}\left(\theta_{\alpha}\right)=\theta_{\beta}
$$

and

$$
\alpha \text { does not survive at } \beta \Rightarrow i_{\alpha \beta}^{\mathscr{T}}\left(\theta_{\alpha}\right)>\theta_{\beta} \text {. }
$$

Definition 4.10 is reasonable because if $\beta_{i} \mathrm{~T} \beta_{i+1}$ and $\beta_{i}$ does not survive at $\beta_{i+1}$, for all $i \in \omega$, then $\left\{\alpha \mid \exists i\left(\alpha \mathrm{T} \beta_{i}\right)\right\}$ is a maximal branch of $\mathscr{T}$ by condition (3) of Definition 4.7. Conversely, if $b$ is a maximal, noncofinal branch of $\mathscr{T}$, then there are arbitrarily large $\beta \in b$ such that $\beta$ does not survive at some $\gamma \in b, \gamma>\beta$. This follows from the fact that if $\gamma=\sup b$, all sufficiently large $\alpha \in[0, \gamma]_{\mathrm{T}}$ survive at $\gamma$. For then there are arbitrarily large $\gamma \in b$ such that for some $\eta \in[0, \lambda]_{\mathrm{T}}$ with $\mathrm{T}-\operatorname{pred}(\gamma)<\eta<\gamma, \eta$ survives at $\lambda$. For such $\gamma$ and $\eta$, the fact that $\eta$ survives at $\lambda$ implies $p(\gamma)>\eta$, using (1) of Definition 4.8. But then (2) of Definition 4.8 implies T-pred $(\gamma)$ does not survive at $\gamma$.

Proof of Lemma 4.6. Suppose the lemma fails, and fix a countable plus two iteration tree $\mathscr{T}$ on a premouse $\mathscr{M}$, together with an elementary $\pi: \mathscr{M} \rightarrow$ $\left(V_{\gamma_{0}}, \in, \delta_{0}\right)$, so that $(\mathscr{T}, \mathscr{M})$ is self-justifying and one of the conclusions of Lemma 4.6 fails. Let $\ln (\mathscr{T})$ be minimal among all such counterexamples $(\mathscr{T}, \mathscr{M}, \pi)$ to Lemma 4.6. We may, and do, assume $\mathscr{M}$ is countable. [Let $\eta$ be large enough that everything relevant is in $V_{\eta}$. Let $\sigma:(Q, \in) \rightarrow\left(V_{\eta}, \in\right)$ be elementary, where $Q$ is countable and transitive and $\mathscr{T}, \mathscr{M} \in \operatorname{ran}(\sigma)$. Let $\sigma(\overline{\mathscr{M}})=\mathscr{M}$ and $\sigma(\overline{\mathscr{T}})=\mathscr{T}$. Then $Q \models$ “( $\overline{\mathscr{T}}, \overline{\mathscr{M}})$ is self-justifying and one of the conclusions of Lemma 4.6 fails for $(\overline{\mathscr{T}}, \bar{M})$ ", so this is in fact true of $(\overline{\mathscr{T}}, \overline{\mathscr{M}})$. On the other hand, $\pi \circ(\sigma \uparrow \overline{\mathscr{M}}): \overline{\mathscr{M}} \rightarrow\left(V_{\gamma_{0}}, \in, \delta\right)$. So we can replace $(\mathscr{T}), \mathscr{M}$ by $(\overline{\mathscr{T}}, \overline{\mathscr{M}})$ and $\pi$ by $\pi \circ(\sigma \uparrow \overline{\mathscr{M}})$.

Fix a preservation function $p$ for the tree order $\mathrm{T}$ associated to $\mathscr{T}$. If $\operatorname{lh}(\mathscr{T})$ is a limit ordinal, then we assume there is a strictly increasing sequence $\left\langle\alpha_{i} \mid i \in \omega\right\rangle$ such that the $\sup _{i \in \omega} \alpha_{i}=\ln (\mathscr{T})$ and $p\left(\alpha_{i+1}+1\right)=\alpha_{i}+1$ and $\forall \eta\left(\alpha_{i}+1<\eta \leq \alpha_{i+1} \Rightarrow \alpha_{i}+1<p(\eta)\right)$ for all $i \in \omega$. The proof of Lemma 4.9 actually constructed such a $p$ in the case $\operatorname{lh}(\mathscr{T})$ is a limit ordinal.

We may assume that $(\mathscr{T}, \mathscr{M})$ is $p$-continuously illfounded. For let $\mathscr{P}=$ $\left(V_{\eta}, \in, \delta_{0}\right)$ be a premouse with $\eta>\gamma_{0}$, and let $\pi \mathscr{T}$ be the copied tree on $\mathscr{P}$. Clearly, $(\pi \mathscr{T}, \mathscr{P})$ is self-justifying. But then since $\mathscr{T}$ has minimal length among all counterexamples to Lemma $4.6, \ln (\pi \mathscr{T})=\operatorname{lh}(\mathscr{T})$. Also, $\pi \mathscr{T}$ is a counterexample to Lemma 4.6 , as this property of $\mathscr{T}$ is carried by $\pi$. Finally, $\pi \mathscr{T}$ is $p$-continuously illfounded. To see this, we argue as before. Fix a map 
$m: \omega \rightarrow\left(\operatorname{lh}(\mathscr{T}) \times \bigcup_{\beta<\operatorname{lh}(\mathscr{T})} \mathscr{M}_{\beta}^{\mathscr{I}}\right)$ such that if $x \in \mathscr{M}_{\beta}^{\mathscr{T}}$, then $m^{-1}((\beta, x))$ is infinite. We form a tree $\mathscr{U}$ in $\mathscr{P}$ by putting $\left(\left\langle\alpha_{0} \cdots \alpha_{n}\right\rangle,\left\langle y_{0} \cdots y_{n}\right\rangle\right) \in \mathscr{U}$ iff (i) $\alpha_{0}=0$, (ii) $\forall i<n\left(\alpha_{i} \mathrm{~T} \alpha_{i+1} \wedge \alpha_{i}\right.$ does not survive at $\left.\alpha_{i+1}\right)$, and (iii) $\left(\mathscr{M}_{\alpha_{n}}^{\mathscr{T}}, z_{0} \cdots z_{n}\right) \equiv\left(V_{\gamma_{0}}, \in, \delta_{0}, y_{0} \cdots y_{n}\right)$, where for $e \leq n, z_{e}=i_{\beta, \alpha_{n}}^{\mathscr{T}}(x)$ if $m(e)=(\beta, x)$ where $\beta \mathrm{T} \alpha_{e}$ and $x \in \mathscr{M}_{\beta}^{\mathscr{T}}$, and $z_{e}=\varnothing$ otherwise. $\mathscr{U}$ is wellfounded because an infinite branch of $\mathscr{U}$ would yield a maximal wellfounded branch of $\mathscr{T}$, and $\mathscr{T}$, being a counterexample to Lemma 4.6, has no such branches. Now let $\pi_{\eta}: \mathscr{M}_{\eta}^{\mathscr{T}} \rightarrow i_{0 \eta}^{\pi \mathscr{T}}\left(\left(V_{\gamma_{0}}, \in, \delta_{0}\right)\right)$ come from the copying construction. Let $\gamma<\operatorname{lh}(\mathscr{T})$; we define an ordinal $\theta_{\gamma} \in \mathscr{M}_{\gamma}^{\pi \mathscr{T}}$. Let $\alpha_{0}=0$, and $\alpha_{i+1}=$ least $\beta \in[0, \gamma]_{\mathrm{T}}$ such that $\alpha_{i} \mathrm{~T} \beta$ and $\alpha_{i}$ does not survive at $\beta$. Let $n$ be largest such that $\alpha_{n}$ is defined. For $e \leq n$ such that $m(e)=(\eta, x)$ for some $\eta \in\left[0, \alpha_{e}\right]_{\mathrm{T}}$ and $x \in \mathscr{M}_{\eta}^{\mathscr{T}}$, set $y_{e}=i_{\eta \gamma}^{\pi \mathscr{T}}\left(\pi_{\eta}(x)\right)$, and for $e \leq n$ otherwise set $y_{e}=\varnothing$. We then let $\theta_{\gamma}$ be the rank in $i_{0 \gamma}^{\pi \mathscr{T}}(\mathscr{U})$ of $\left\langle\left(\alpha_{i}, y_{i}\right) \mid i \leq n\right\rangle$. One can easily check that the $\theta_{\gamma}$ 's witness the $p$-continuous illfoundedness of $(\pi \mathscr{T}, \mathscr{P})$. Finally, we can reduce $\mathscr{P}$ again to a countable model: let $\sigma:(Q, \in) \rightarrow\left(V_{\xi}, \in\right)$ where $Q$ is transitive, $\xi$ is large, and $\mathscr{P}$, $\pi \mathscr{T}, p \in \operatorname{ran}(\sigma)$. Then $\sigma^{-1}(\pi \mathscr{T})$ is a self-justifying plus two tree on $\sigma^{-1}$ $(\mathscr{P})$ which is a counterexample to one of the conclusions of Lemma 4.6 and is $\sigma^{-1}(p)=p$ continuously illfounded. Moreover, $\sigma \uparrow \sigma^{-1}(\mathscr{P}): \sigma^{-1}(\mathscr{P}) \rightarrow \mathscr{P}=$ $\left(V_{\eta}, \in, \delta_{0}\right)$. So we can replace $\mathscr{M}$ by $\sigma^{-1}(\mathscr{P}), \mathscr{T}$ by $\sigma^{-1}(\pi \mathscr{T})$, and $\pi$ by $\sigma \uparrow \sigma^{-1}(\mathscr{P})$.

To summarize, we now have a countable premouse $\mathscr{M}$, an embedding $\pi$ : $\mathscr{M} \rightarrow\left(V_{\gamma_{0}}, \in, \delta_{0}\right)$, and a countable plus two iteration tree $\mathscr{T}$ on $\mathscr{M}$ such that $(\mathscr{T}, \mathscr{M})$ is $p$-continuously illfounded for a preservation function $p$ having a certain additional property if $\operatorname{lh}(\mathscr{T})$ is a limit. Moreover (by minimality), if $\operatorname{lh}(\mathscr{T})=\gamma+1$ for some $\gamma$, then there is an extender $E \in \mathscr{M}_{\gamma}^{\mathscr{T}}$ and $\beta \leq \gamma$ such that $\operatorname{crit}(E)+1<\rho^{\mathscr{T}}(\beta, \gamma)$ and $\operatorname{Ult}\left(\mathscr{M}_{\beta}^{\mathscr{T}}, E\right)$ is illfounded. From this we shall derive a contradiction.

The following notation will be useful. Suppose $\ln (\mathscr{T})=\gamma+1$, and fix $E \in \mathscr{M}_{\gamma}^{\mathscr{T}}$ such that $\operatorname{crit}(E)+1<\rho^{\mathscr{T}}(\beta, \gamma)$ and $\operatorname{Ult}\left(\mathscr{M}_{\beta}^{\mathscr{T}}, E\right)$ is illfounded. Let $\kappa=\operatorname{crit}(E)$, and set

$$
g(\eta)= \begin{cases}\text { least successor ordinal } \geq \mu^{\mathscr{T}}(\eta) & \text { if } \eta<\beta, \\ \text { least successor ordinal } \geq \max \left(\mu^{\mathscr{T}}(\eta), \kappa\right) & \text { if } \beta \leq \eta<\gamma .\end{cases}
$$

Then for $\alpha<\delta \leq \gamma$, we set

$$
\mu^{\mathscr{T}}(\alpha, \delta)=\inf \{g(\eta) \mid \alpha \leq \eta<\delta\} .
$$

Let us call a plus one enlargement $\left\langle\left(\pi_{\alpha}, \mathscr{P}_{\alpha}, \nu_{\alpha}\right) \mid \alpha \leq \lambda\right\rangle$ of $(\mathscr{T}, \mathscr{M})$ good if $\pi_{\alpha}$ agrees with $\pi_{\lambda}$ through $\mu^{\mathscr{G}}(\alpha, \lambda)$ for all $\alpha<\lambda$.

If $\operatorname{lh}(\mathscr{T})$ is a limit ordinal, we let $\mu^{\mathscr{T}}(\alpha, \delta)=\inf \{g(\eta) \mid \alpha \leq \eta<\delta\}$, where $g(\eta)$ is the least successor ordinal $\geq \mu^{\mathscr{T}}(\eta)$ for all $\eta$. In this case, if 
$\left\langle\left(\pi_{\alpha}, \mathscr{P}_{\alpha}, \nu_{\alpha}\right) \mid \alpha \leq \lambda\right\rangle$ is any plus one enlargement, then $\pi_{\alpha}$ agrees with $\pi_{\lambda}$ through $\mu^{\mathscr{T}}(\alpha, \lambda)$ for all $\alpha<\lambda$; thus any plus one enlargement is good.

Notice that in either case $g(\eta)<\rho^{\mathscr{T}}(\eta, \eta+1)$ for all $\eta$ because $\mathscr{T}$ is a plus two tree.

We shall obtain our contradiction from a certain enlargement of $(\mathscr{T}, \mathscr{M})$, which we construct as a "limit" of a sequence $\left\langle\mathscr{E}^{\alpha} \mid \alpha<\operatorname{lh}(\mathscr{T})\right\rangle$ of enlargements of $(\mathscr{T}, \mathscr{M}), \mathscr{E}^{\alpha}$ being of length $\alpha+1$. Before going to the construction, we show that no illfounded ultrapowers will arise in the course of it. To that end, we must record some properties of the sequence $\left\langle\mathscr{E}^{\alpha} \mid \alpha<\operatorname{lh}(\mathscr{T})\right\rangle$ we are about to construct-in fact, nearly all its properties.

Definition 4.11. Let $\lambda \leq \ln (\mathscr{T})$, and for $\alpha<\lambda$ let $\mathscr{E}^{\alpha}=\left\langle\left(\pi_{\beta}^{\alpha}, \mathscr{P}_{\beta}^{\alpha}, \nu_{\beta}^{\alpha}\right)\right|$ $\beta \leq \alpha\rangle$ be a good plus one enlargement of $(\mathscr{T}, \mathscr{M})$. We call the sequence $\left\langle\mathscr{E}^{\alpha} \mid \alpha<\lambda\right\rangle$ connected iff there are elementary $j_{\alpha \delta}: \mathscr{P}_{\alpha}^{\alpha} \rightarrow \mathscr{P}_{\delta}^{\delta}$ defined when $\alpha$ survives at $\delta$ such that the following conditions hold:

(0) For $\alpha+1<\lambda, \mathscr{E}^{\alpha+1} \mid \alpha+1$ is also a good plus one enlargement of $(\mathscr{T}, \mathscr{M})$. Moreover, $\nu_{\beta}^{\alpha} \in \mathscr{P}_{\beta}^{\alpha}$, for $\beta \leq \alpha<\lambda$.

(1) Let $p(\alpha+1)=\beta+1$, where $\alpha+1<\lambda$. Then

(i) $\mathscr{E}_{\gamma}^{\alpha+1}=\mathscr{E}_{\gamma}^{\alpha}$ for $\gamma \leq \beta$;

(ii) if $\beta<\alpha$, then letting $\rho=\pi_{\alpha}^{\alpha}\left(\mu^{\mathscr{T}}(\beta, \alpha)\right)$ we have

(a) $V_{\rho+1}^{\mathscr{P}_{\alpha}^{\alpha}} \subseteq \mathscr{P}_{\alpha}^{\alpha+1}$, and

(b) $\mathscr{P}_{\alpha}^{\alpha+1} \in \mathscr{P}_{\alpha}^{\alpha}$ and $\mathscr{P}_{\alpha}^{\alpha} \models \mathscr{P}_{\alpha}^{\alpha+1}$ has the same cardinality as $V_{\rho+1}$ ",

(c) for some $\eta>\nu_{\alpha}^{\alpha}, \quad \eta \in \mathscr{P}_{\alpha}^{\alpha}$, there is an elementary $\pi$ : $\mathscr{P}_{\alpha}^{\alpha+1} \rightarrow\left(V_{\eta}^{\mathscr{P}_{\alpha}^{\alpha}}, \in, \delta^{\mathscr{P}_{\alpha}^{\alpha}}\right)$ such that $\pi \uparrow V_{\rho+1}^{\mathscr{P}_{\alpha}^{\alpha}}=$ identity, $\operatorname{ran}\left(\pi_{\alpha}^{\alpha}\right) \subseteq \operatorname{ran}(\pi)$, and $\pi_{\alpha}^{\alpha+1}=\pi^{-1} \circ \pi_{\alpha}^{\alpha}$;

(iii) if $\beta+1<\alpha$, then there is a sequence $\left\langle\left(\mathscr{R}_{\gamma}, \eta_{\gamma}^{i}\right)\right| \beta<\gamma<\alpha$ and $0 \leq i<\omega\rangle \in \mathscr{P}_{\alpha}^{\alpha+1}$ such that for $\beta<\gamma<\alpha$ and $i \in \omega$

(a) $\eta_{\gamma}^{i}<\eta_{\gamma}^{i+1} \in \mathscr{R}_{\gamma}$,

(b) $\mathscr{R}_{\gamma}$ is a premouse, and $\left(V_{\eta_{\gamma}^{i}}^{\mathscr{R}_{\gamma}}, \in, \delta^{\mathscr{R}_{\gamma}}\right)$ is a premouse,

(c) $\mathscr{P}_{\gamma}^{\alpha+1}=\left(V_{\eta_{\gamma}^{0}}^{\mathscr{R}_{\gamma}}, \in, \delta^{\mathscr{R}_{\gamma}}\right)$, and

(d) $\pi_{\gamma}^{\alpha+1}$ agrees with $\pi_{\alpha}^{\alpha+1}$ through $\mu^{\mathscr{T}}(\gamma, \alpha)$;

(iv) let $\delta=\mathrm{T}$-pred $(\alpha+1)$; then

$$
\mathscr{P}_{\alpha+1}^{\alpha+1}=\operatorname{Ult}\left(\mathscr{P}_{\delta}^{\alpha+1}, \pi_{\alpha}^{\alpha+1}\left(E_{\alpha}^{\mathscr{T}}\right)\right),
$$

and if $i: \mathscr{P}_{\delta}^{\alpha+1} \rightarrow \mathscr{P}_{\alpha+1}^{\alpha+1}$ is the canonical embedding, then

$$
\nu_{\alpha+1}^{\alpha+1}=i\left(\nu_{\delta}^{\alpha+1}\right)
$$

and if $\delta$ survives at $\alpha+1$, then $j_{\delta, \alpha+1}=i$ and $j_{\gamma, \alpha+1}=j_{\delta, \alpha+1} \circ j_{\gamma, \delta}$ for $\gamma \mathrm{T} \delta, \gamma$ surviving at $\delta$.

(2) Let $\alpha<\lambda$ be a limit. Then 
(i) $\mathscr{P}_{\alpha}^{\alpha}$ is the direct limit of the $\mathscr{P}_{\beta}^{\beta}$, for $\beta \mathrm{T} \alpha$ such that $\beta$ survives at $\alpha$, under the $j_{\beta \delta}$ 's for $\beta$ surviving at $\alpha, j_{\beta \alpha}: \mathscr{P}_{\beta}^{\beta} \rightarrow \mathscr{P}_{\alpha}^{\alpha}$ is the canonical embedding; $\nu_{\alpha}^{\alpha}$ is the common value of $j_{\beta \alpha}\left(\nu_{\beta}^{\beta}\right)$ for all $\beta$ surviving at $\alpha$;

(ii) (Pointwise eventual commutativity) if $\beta \mathrm{T} \alpha$ and $x \in \mathscr{M}_{\beta}^{\mathscr{T}}$, then for all sufficiently large $\gamma \mathrm{T} \alpha$, whenever $\gamma \mathrm{T} \delta \mathrm{T} \alpha$ we have $j_{\gamma \delta}\left(\pi_{\gamma}^{\gamma}\left(i_{\beta \gamma}^{\mathscr{T}}(x)\right)\right)=$ $\pi_{\delta}^{\delta}\left(i_{\beta \delta}^{\mathscr{T}}(x)\right)$;

(iii) for $\gamma<p(\alpha), \mathscr{E}_{\gamma}^{\alpha}=$ eventual value of $\mathscr{E}_{\gamma}^{\beta}$ as $\beta \rightarrow \alpha$;

(iv) for some $\left\langle\left(\mathscr{R}_{\beta}, \eta_{\beta}^{i}\right)\right| p(\alpha) \leq \beta<\alpha$ and $\left.i \in \omega\right\rangle$ belonging to $\mathscr{P}_{\alpha}^{\alpha}$, we have

(a) $\eta_{\beta}^{i}<\eta_{\beta}^{i+1} \in \mathscr{R}_{\beta}$, for all $\beta, i$,

(b) $\mathscr{R}_{\beta}$ is a premouse, and $\left(V_{\eta_{\beta}^{i}}^{\mathscr{R}_{\beta}}, \in, \delta^{\mathscr{R}_{\beta}}\right)$ is a premouse, for all $\beta, i$,

(c) $\mathscr{P}_{\beta}^{\alpha}=\left(V_{\eta_{\beta}^{0}}^{\mathscr{R}_{\beta}}, \in, \delta^{\mathscr{R}_{\beta}}\right)$, for all $\beta$.

Remarks. 1. If $\alpha<\beta$ and $p(\eta)>\gamma$ for all $\eta$ such that $\alpha<\eta \leq \beta$, then $\mathscr{E}_{\gamma}^{\alpha}=\mathscr{E}_{\gamma}^{\beta}$. Thus the eventual value referred to in (2)(iii) exists.

2. The $j_{\alpha \beta}$ 's are uniquely determined by $\left\langle\mathscr{E}^{\alpha} \mid \alpha<\lambda\right\rangle$.

3. Let $\left\langle\mathscr{E}^{\alpha} \mid \alpha<\lambda\right\rangle$ be connected, and suppose $\beta<\alpha<\lambda$ and $\forall \eta(\beta<\eta \leq$ $\alpha \Rightarrow \beta<p(\eta))$. Let $\rho=\pi_{\beta}^{\beta}\left(\mu^{\mathscr{T}}(\beta, \alpha)\right)=\pi_{\beta}^{\alpha}\left(\mu^{\mathscr{T}}(\beta, \alpha)\right)=\pi_{\alpha}^{\alpha}\left(\mu^{\mathscr{T}}(\beta, \alpha)\right)$. Then

moreover,

$$
V_{\rho+1}^{\mathscr{P}_{\alpha}^{\alpha}}=V_{\rho+1}^{\mathscr{P}_{\beta}^{\beta}} \quad \text { and } \quad V_{\rho+2}^{\mathscr{P}_{\alpha}^{\alpha}} \subseteq V_{\rho+2}^{\mathscr{P}_{\beta}^{\beta}}
$$

$$
V_{\rho+1}^{\mathscr{P}_{\gamma}^{\alpha}} \subseteq V_{\rho+1}^{\mathscr{P}_{\beta}^{\beta}} \quad \text { for } \beta<\gamma<\alpha .
$$

[Fix $\beta$; the proof is by induction on $\alpha$. Call the assertion to be proved $\left(^{*}\right)$.

$\alpha$ limit. Notice first that for all sufficiently large $\delta+1 \in[0, \alpha)_{\mathrm{T}}, \operatorname{crit}\left(E_{\delta}^{\mathscr{I}}\right)>$ $\mu^{\mathscr{T}}(\beta, \alpha)$. For if $\beta \leq \delta<\alpha$, then $\mu^{\mathscr{T}}(\beta, \alpha) \leq g(\delta)<\rho^{\mathscr{T}}(\delta, \delta+1)=$ strength of $E_{\delta}^{\mathscr{T}}$ in $\mathscr{M}_{\delta}^{\bar{T}}$. But all our extenders have strength less than or equal to the image of the critical point, so if $\operatorname{crit}\left(E_{\delta}^{\mathscr{G}}\right) \leq \mu^{\mathscr{T}}(\beta, \alpha)$ for arbitrarily large $\delta+1 \in[0, \alpha)_{\mathrm{T}}$ then $\mathscr{M}_{\alpha}^{\mathscr{T}}$ is illfounded, a contradiction. Now let $\eta \in[0, \alpha)_{\mathrm{T}}$ be large enough that $\beta<\eta, \eta$ survives at $\alpha$, and $\operatorname{crit}\left(E_{\delta}^{\mathscr{I}}\right)>$ $\mu^{\mathscr{T}}(\beta, \alpha)$ whenever $\delta+1 \in[\eta, \alpha)_{\mathrm{T}}$. So $\mathscr{P}_{\alpha}^{\alpha}$ is the direct limit of the $\mathscr{P}_{\xi}^{\xi}$, for $\xi \in[\eta, \alpha)_{\mathrm{T}}$, under the $j_{\delta \xi}$ 's. If $\xi, \delta+1 \in[\eta, \alpha)_{\mathrm{T}}$ and T-pred $(\delta+1)$ $=\xi$, then $\mathscr{P}_{\delta+1}^{\delta+1}=\operatorname{Ult}\left(\mathscr{P}_{\xi}^{\xi}, \pi_{\delta}^{\delta+1}\left(E_{\delta}^{\mathscr{T}}\right)\right)$. But then $\operatorname{crit}\left(\pi_{\delta}^{\delta+1}\left(E_{\delta}^{\mathscr{T}}\right)\right)>$ $\pi_{\delta}^{\delta+1}\left(\mu^{\mathscr{T}}(\beta, \alpha)\right)=\pi_{\beta}^{\delta+1}\left(\mu^{\mathscr{T}}(\beta, \alpha)\right)\left(\right.$ since $\left.\mu^{\mathscr{T}}(\beta, \alpha) \leq \mu^{\mathscr{T}}(\beta, \delta)\right)=$ $\pi_{\beta}^{\beta}\left(\mu^{\mathscr{T}}(\beta, \alpha)\right)$ (since $p(\eta)>\beta$ for $\left.\beta<\eta \leq \alpha\right)=\rho$. Thus $\operatorname{crit}\left(j_{\eta \alpha}\right)>\rho$, so that $V_{\rho+1}^{\mathscr{P}_{\alpha}^{\alpha}}=V_{\rho+1}^{\mathscr{P}_{\eta}^{\eta}}=V_{\rho+1}^{\mathscr{P}_{\beta}^{\beta}}$ and $V_{\rho+2}^{\mathscr{P}_{\alpha}^{\alpha}}=V_{\rho+2}^{\mathscr{P}_{\eta}^{\eta}} \subseteq V_{\rho+2}^{\mathscr{P}_{\beta}^{\beta}}$ by induction. Finally, if $\gamma<\alpha$, then either $\mathscr{P}_{\gamma}^{\alpha}=\mathscr{P}_{\gamma}^{\delta}$ for $\delta<\alpha$ sufficiently large, so we can apply induction, or $\mathscr{P}_{\gamma}^{\alpha} \in \mathscr{P}_{\alpha}^{\alpha}$, so we can apply $\left(^{*}\right)$ for $\mathscr{P}_{\alpha}^{\alpha}$. 
$\alpha$ successor. Let $\delta=\mathrm{T}-\operatorname{pred}(\alpha)$ so that $\mathscr{P}_{\alpha}^{\alpha}=\operatorname{Ult}\left(\mathscr{P}_{\delta}^{\alpha}, \pi_{\alpha-1}^{\alpha}\left(E_{\alpha-1}^{\mathscr{I}}\right)\right)$. Because $\mathscr{T}$ is a plus two tree, $g(\alpha-1)+1 \leq \rho^{\mathscr{T}}(\alpha-1, \alpha)$. Thus $\mathscr{P}_{\alpha}^{\alpha}$ agrees with $\mathscr{P}_{\alpha-1}^{\alpha}$ through $\pi_{\alpha-1}^{\alpha}(g(\alpha-1))+1$. But $\mu^{\mathscr{T}}(\beta, \alpha) \leq g(\alpha-1)$, and $\pi_{\alpha-1}^{\alpha}\left(\mu^{\mathscr{T}}(\beta, \alpha)\right)=\pi_{\beta}^{\alpha}\left(\mu^{\mathscr{T}}(\beta, \alpha)\right)=\pi_{\beta}^{\beta}\left(\mu^{\mathscr{T}}(\beta, \alpha)\right)=\rho$. (To see that $\pi_{\alpha-1}^{\alpha}\left(\mu^{\mathscr{T}}(\beta, \alpha)\right)=\pi_{\beta}^{\alpha}\left(\mu^{\mathscr{T}}(\beta, \alpha)\right)$, notice that $\mathscr{E}^{\alpha} \uparrow \alpha$ is an enlargement and $\mu^{\mathscr{T}}(\beta, \alpha) \leq \mu^{\mathscr{T}}(\beta, \alpha-1)$.) So $\mathscr{P}_{\alpha}^{\alpha}$ agrees with $\mathscr{P}_{\alpha-1}^{\alpha}$ through $\rho+1$ and $V_{\rho+2}^{\mathscr{P}_{\alpha}^{\alpha}} \subseteq \mathscr{P}_{\alpha-1}^{\alpha}$. But now $\mathscr{P}_{\alpha-1}^{\alpha}=\mathscr{P}_{\alpha-1}^{\alpha-1}$ if $p(\alpha)=\alpha$. If $p(\alpha)<\alpha$, then setting $\nu=\pi_{\alpha-1}^{\alpha-1}\left(\mu^{\mathscr{T}}(p(\alpha)-1, \alpha-1)\right)$ we see from (1)(ii) of Definition 4.11 that $\mathscr{P}_{\alpha-1}^{\alpha}$ agrees with $\mathscr{P}_{\alpha-1}^{\alpha-1}$ through $\nu+1$ and that $V_{\nu+2}^{\mathscr{P}_{\alpha-1}^{\alpha}} \subseteq \mathscr{P}_{\alpha-1}^{\alpha-1}$. We see also that $\pi_{\alpha-1}^{\alpha}\left(\mu^{\mathscr{T}}(p(\alpha)-1, \alpha-1)\right)=\nu$. Since $\beta \leq p(\alpha)-1, \mu^{\mathscr{T}}(\beta, \alpha) \leq$ $\mu^{\mathscr{T}}(p(\alpha)-1, \alpha-1)$, and thus $\rho \leq \nu$. It follows that $\mathscr{P}_{\alpha}^{\alpha}$ agrees with $\mathscr{P}_{\alpha-1}^{\alpha-1}$ through $\rho+1$ and that $V_{\rho+2}^{\mathscr{P}_{\alpha}^{\alpha}} \subseteq \mathscr{P}_{\alpha-1}^{\alpha-1}$. By induction then, $\mathscr{P}_{\alpha}^{\alpha}$ has the desired agreement with $\mathscr{P}_{\beta}^{\beta}$.

Finally, if $\gamma<\alpha-1$ then either $\mathscr{P}_{\gamma}^{\alpha}=\mathscr{P}_{\gamma}^{\alpha-1}$ or $\mathscr{P}_{\gamma}^{\alpha} \in \mathscr{P}_{\alpha-1}^{\alpha}$, and in either case $\left(^{*}\right)$ holds for $\mathscr{P}_{\gamma}^{\alpha}$.]

4. Suppose $\beta=\operatorname{T-pred}(\alpha)$ and $\beta$ survives at $\alpha$. Let $\left\langle\mathscr{E}^{\eta} \mid \eta<\lambda\right\rangle$ be a connected sequence of enlargements. Then $j_{\beta \alpha}: \mathscr{P}_{\beta}^{\beta} \rightarrow \mathscr{P}_{\alpha}^{\alpha}$ is internal; that is, it comes from an ultrapower by the extender belonging to $\mathscr{P}_{\beta}^{\beta}$. For $\mathscr{P}_{\alpha}^{\alpha}=$ $\operatorname{Ult}\left(\mathscr{P}_{\beta}^{\beta}, \pi_{\alpha-1}^{\alpha}\left(E_{\alpha-1}^{\mathscr{T}}\right)\right)$, and $j_{\beta \alpha}$ is the canonical embedding. If $\alpha-1=\beta$, then $\pi_{\alpha-1}^{\alpha}\left(E_{\alpha-1}^{\mathscr{T}}\right) \in \mathscr{P}_{\beta}^{\alpha}=\mathscr{P}_{\beta}^{\beta}$. If $\alpha-1 \geq \beta+1$, then as $p(\alpha)=\beta+1$ we see from (1)(ii) of Definition 4.11 that $\mathscr{P}_{\alpha-1}^{\alpha}$ is coded by a set $A \in V_{\rho+1}^{\mathscr{P}_{\alpha-1}^{\alpha-1}}$, where $\rho=\pi_{\alpha-1}^{\alpha-1}\left(\mu^{\mathscr{T}}(\beta, \alpha-1)\right)$. By remark $3, A \in \mathscr{P}_{\beta}^{\beta}$, so $\mathscr{P}_{\alpha-1}^{\alpha} \in \mathscr{P}_{\beta}^{\beta}$, so $\pi_{\alpha-1}^{\alpha}\left(E_{\alpha-1}^{\mathscr{T}}\right) \in \mathscr{P}_{\beta}^{\beta}$.

5. From remark 4 we see that the direct limit yielding $\mathscr{P}_{\alpha}^{\alpha}$ in the case $\alpha$ is a limit (Definition $4.11(2)(\mathrm{i})$ ) is the limit of an internal iteration. So in constructing a connected sequence of enlargements, we need not worry directly about the wellfoundedness of $\mathscr{P}_{\alpha}^{\alpha}, \alpha$ limit.

We can now prove the sublemma for whose sake we have isolated the notion of a connected sequence of enlargements.

Sublemma 4.12. Let $\left\langle\mathscr{E}^{\alpha} \mid \alpha \leq \theta\right\rangle$ be a connected sequence of enlargements of $(\mathscr{T}, \mathscr{M})$. Say $\mathscr{E}^{\alpha}=\left\langle\left(\pi_{\beta}^{\alpha}, \mathscr{P}_{\beta}^{\alpha}, \nu_{\beta}^{\alpha}\right) \mid \beta \leq \alpha\right\rangle$. Suppose $\mathscr{P}_{0}^{0}$ is elementarily embeddable into some $\left(V_{\eta}, \in, \delta\right)$. Suppose $\mathscr{P}_{\theta}^{\theta} \models$ “ $E$ is an extender", and suppose crit $(E)<\pi_{\theta}^{\theta}\left(\mu^{\mathscr{T}}(\beta, \theta)\right)$, where $\beta<\theta$. Then $\operatorname{Ult}\left(\mathscr{P}_{\beta}^{\theta}, E\right)$ is wellfounded.

Proof. Let $\theta$ be least such that the sublemma is false for some sequence $\left\langle\mathscr{E}^{\alpha}\right|$ $\alpha \leq \theta\rangle$ of length $\theta+1$. By taking a Skolem hull, we can find a counterexample $\left(\left\langle\mathscr{E}^{\alpha} \mid \alpha \leq \theta\right\rangle, E\right)$ to the sublemma such that all the $\mathscr{P}_{\beta}^{\alpha}, \beta \leq \alpha \leq \theta$, are 
countable. (See, e.g., the proof of Theorem 3.7.) So we fix such a counterexample.

Our plan, as in the proof of Theorem 3.7, is to construct wellfounded premice $\mathscr{R}$ and $\mathscr{S}$, together with elementary embeddings $\pi: \mathscr{P}_{\theta}^{\theta} \rightarrow\left(V_{\mu}^{\mathscr{R}}, \in, \delta^{\mathscr{R}}\right)$ and $\sigma: \mathscr{P}_{\beta}^{\theta} \rightarrow \mathscr{S}$ such that $\pi \nmid V_{\rho}^{\mathscr{P}_{\theta}^{\theta}}=\sigma \nmid V_{\rho}^{\mathscr{P}_{\beta}^{\theta}}$, where $\rho=\pi_{\theta}^{\theta}\left(\mu^{\mathscr{G}}(\beta, \theta)\right)=$ $\pi_{\beta}^{\theta}\left(\mu^{\mathscr{T}}(\beta, \theta)\right), \pi \in \mathscr{R}$, and $\mathscr{R} \models$ “ $\mathscr{P}_{\theta}^{\theta}$ is countable". This is a contradiction. (There is a sequence $\left\langle\bar{A}_{i} \mid i \in \omega\right\rangle$, with $\bar{A}_{i} \in E_{a_{i}}$, such that $\left\langle\sigma\left(\bar{A}_{i}\right) \mid i \in \omega\right\rangle$ has empty "intersection". But $\left\langle\left\langle\bar{A}_{i}, a_{i}\right\rangle \mid i \in \omega\right\rangle \in \mathscr{R}$ since in fact $V_{\omega+1} \subseteq \mathscr{R}$, and $\pi \in \mathscr{R}$, and $\pi\left(\bar{A}_{i}\right)=\sigma\left(\bar{A}_{i}\right)$. It follows that $\mathscr{R} \models$ “ $\pi(E)$ is not $\omega$-complete", a contradiction. See the proof of Theorem 3.7 for more detail.)

Fix maps $n: \theta \stackrel{1-1}{\longrightarrow} \omega$ and $m: \omega \stackrel{\text { onto }}{\longrightarrow}\left\{(\beta, x) \mid \beta \leq \theta \wedge x \in \mathscr{P}_{\beta}^{\beta}\right\}$. We now define, by induction on $\beta \leq \theta$, premice $Q_{\alpha}^{\beta}$, embeddings $\sigma_{\alpha}^{\beta}$ and ordinals $\mu_{\alpha}^{\beta}$, for $\alpha \leq \beta$, such that the following conditions hold.

(1) $\sigma_{\alpha}^{\beta}: \mathscr{P}_{\alpha}^{\beta} \rightarrow\left(V_{\mu}^{Q}, \in, \delta^{Q}\right)$ elementarily, where $\mu=\mu_{\alpha}^{\beta} \in Q=Q_{\alpha}^{\beta}$.

(2) $\left\langle\left(\sigma_{\alpha}^{\gamma} \circ \pi_{\alpha}^{\gamma}, Q_{\alpha}^{\gamma}, \sigma_{\alpha}^{\gamma}\left(\nu_{\alpha}^{\gamma}\right)\right) \mid \alpha \leq \gamma \leq \beta\right\rangle$ is a connected sequence of enlargements of $(\mathscr{T}, \mathscr{M})$. Moreover, if $\alpha<\beta$, then $\sigma_{\alpha}^{\beta}$ agrees with $\sigma_{\beta}^{\beta}$ through $\rho$, where $\rho=\pi_{\alpha}^{\beta}\left(\mu^{\mathscr{T}}(\alpha, \beta)\right)=\pi_{\beta}^{\beta}\left(\mu^{\mathscr{T}}(\alpha, \beta)\right)$.

For $\alpha$ surviving at $\beta$ let $j_{\alpha \beta}: \mathscr{P}_{\alpha}^{\alpha} \rightarrow \mathscr{P}_{\beta}^{\beta}$ and $k_{\alpha \beta}: Q_{\alpha}^{\alpha} \rightarrow Q_{\beta}^{\beta}$ be the maps given by connectedness.

(3) (Commutativity at small indices) Suppose $m(e)=(\alpha, x)$ where $\alpha$ survives at $\beta$, and suppose $\gamma \in[\alpha, \beta)_{\mathrm{T}}$ is such that $e \leq n(\xi)$ for all $\xi \in$ $(\gamma, \beta]_{\mathrm{T}}$. Then letting $y=j_{\alpha \gamma}(x)$, we have $k_{\gamma \beta}\left(\sigma_{\gamma}^{\gamma}(y)\right)=\sigma_{\beta}^{\beta}\left(j_{\gamma \beta}(y)\right)$.

(4) (Commutativity at small ranks) Suppose $\alpha$ survives at $\beta$, and let $\delta$ be small enough that $\delta<p(\gamma)$ whenever $\alpha<\gamma \leq \beta$. Let $\rho=\pi_{\alpha}^{\alpha}\left(\mu^{\mathscr{F}}(\delta, \beta)\right)=$ $\pi_{\beta}^{\beta}\left(\mu^{\mathscr{G}}(\delta, \beta)\right)$, and $x \in V_{\rho}^{\mathscr{P}^{\alpha}}$. Suppose that $\operatorname{crit}\left(j_{\alpha \beta}\right)>\rho$ and $\operatorname{crit}\left(k_{\alpha \beta}\right)>$ $\sigma_{\alpha}^{\alpha}(\rho)$. Then $k_{\alpha \beta}\left(\sigma_{\alpha}^{\alpha}(x)\right)=\sigma_{\beta}^{\beta}\left(j_{\alpha \beta}(x)\right)$.

(5) $\sigma_{\beta}^{\beta} \in Q_{\beta}^{\beta}$; moreover, $Q_{\beta}^{\beta} \models$ “ $\mathscr{P}_{\beta}^{\beta}$ is countable", and in fact $V_{\kappa} \in Q_{\alpha}^{\beta}$, where $\kappa$ is the least measurable cardinal, for all $\alpha, \beta$.

If we define such $Q_{\alpha}^{\beta}, \sigma_{\alpha}^{\beta}$, and $\mu_{\alpha}^{\beta}$ then we shall have carried out our plan, for we can set $\mathscr{R}=Q_{\theta}^{\theta}, \mu=\mu_{\theta}^{\theta}$, and $\mathscr{S}=\left(V_{\nu}^{Q}, \in, \delta^{Q}\right)$ where $\nu=\mu_{\beta}^{\theta}$ and $Q=Q_{\beta}^{\theta}, \pi=\sigma_{\theta}^{\theta}$, and $\sigma=\sigma_{\beta}^{\theta}$.

$\beta=0$. By hypothesis we have an elementary $\pi: \mathscr{P}_{0}^{0} \rightarrow\left(V_{\eta}, \in, \delta\right)$. Let $\gamma>\eta$ be such that $\left(V_{\gamma}, \in, \delta\right)$ is a premouse, and set

$$
\begin{aligned}
& Q_{0}^{0}=\left(V_{\gamma}, \in, \delta\right), \\
& \sigma_{0}^{0}=\pi, \\
& \mu_{0}^{0}=\eta .
\end{aligned}
$$


$\beta$ successor. We follow (1) of Definition 4.11.

(i) For $\alpha<p(\beta)$, set $\left(\sigma_{\alpha}^{\beta}, Q_{\alpha}^{\beta}, \mu_{\alpha}^{\beta}\right)=\left(\sigma_{\alpha}^{\beta-1}, Q_{\alpha}^{\beta-1}, \mu_{\alpha}^{\beta-1}\right)$.

(ii) Suppose $p(\beta)<\beta$, and let $\rho=\pi_{\beta-1}^{\beta-1}\left(\mu^{\mathscr{T}}(p(\beta)-1, \beta-1)\right)$. Let

$$
\pi: \mathscr{P}_{\beta-1}^{\beta} \longrightarrow\left(V_{\eta}^{\mathscr{P}_{\beta-1}^{\beta-1}}, \in, \delta^{\mathscr{P}_{\beta-1}^{\beta-1}}\right)
$$

be as in (1)(ii) of 4.11. (So $\mathscr{P}_{\beta-1}^{\beta} \in \mathscr{P}_{\beta-1}^{\beta-1}$, has cardinality that of $V_{\rho+1}$ there, and $\pi \uparrow V_{\rho+1}^{\mathscr{P}_{\beta-1}^{\beta-1}}$ is the identity.) Let $\eta^{*}=\sigma_{\beta-1}^{\beta-1}(\eta), \rho^{*}=\sigma_{\beta-1}^{\beta-1}(\rho)$, and $\mu=\mu_{\beta-1}^{\beta-1}$. We define

$$
\begin{aligned}
Q_{\beta-1}^{\beta}= & \text { transitive collapse of a Skolem closure of } \\
& V_{\rho^{*}+1}^{Q_{\beta-1}^{\beta-1}} \cup\left\{\eta^{*}, \sigma_{\beta-1}^{\beta-1} \uparrow V_{\eta}^{\mathscr{P}_{\beta-1}^{\beta-1}}\right\} \text { inside } V_{\mu}^{Q_{\beta-1}^{\beta-1}} .
\end{aligned}
$$

Let $\tau: Q_{\beta-1}^{\beta} \longrightarrow V_{\mu}^{Q_{\beta-1}^{\beta-1}}$ be the inverse of the collapse. Set

$$
\begin{aligned}
\mu_{\beta-1}^{\beta} & =\tau^{-1}\left(\eta^{*}\right), \\
\sigma_{\beta-1}^{\beta} & =\tau^{-1}\left(\sigma_{\beta-1}^{\beta-1} \uparrow V_{\eta}^{\mathscr{P}_{\beta-1}^{\beta-1}}\right) \circ \pi \\
& =\tau^{-1} \circ \sigma_{\beta-1}^{\beta-1} \circ \pi .
\end{aligned}
$$

The following commutative diagram involving the models and embeddings just defined may be helpful.

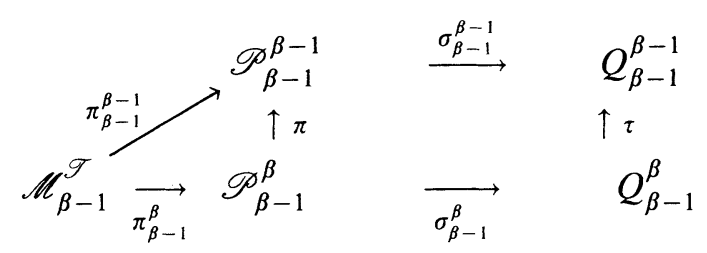

In all cases, the embeddings are actually into a rank of the range model.

(iii) Suppose $p(\beta)<\beta-1$. By (1)(iii) of Definition 4.11 we have a sequence

$$
\left.\left.\left\langle\left(\mathscr{R}_{\gamma}, \eta_{\gamma}^{i}\right)\right| p(\beta) \leq \gamma<\beta-1 \wedge i \in \omega\right)\right\rangle \in \mathscr{P}_{\beta-1}^{\beta}
$$

such that $\mathscr{P}_{\gamma}^{\beta}=V_{\eta_{y}^{0}}^{\mathscr{R}_{y}}$ for $p(\beta) \leq \gamma<\beta-1$. We define

$$
\begin{aligned}
Q_{\gamma}^{\beta} & =\sigma_{\beta-1}^{\beta}\left(\left(V_{\eta_{\gamma}^{1}}^{\mathscr{R}_{\gamma}}, \in, \delta^{\mathscr{R}_{\gamma}}\right)\right), \\
\mu_{\gamma}^{\beta} & =\sigma_{\beta-1}^{\beta}\left(\eta_{\gamma}^{0}\right),
\end{aligned}
$$

and

for $p(\beta) \leq \gamma<\beta-1$.

$$
\sigma_{\gamma}^{\beta}=\sigma_{\beta-1}^{\beta}\left\lceil\mathscr{P}_{\gamma}^{\beta}\right.
$$

Remark. This is the point at which we use that there are $\omega$-many $\eta_{\gamma}$ 's, for each $\gamma$. The sequence verifying (1)(iii) for the $Q_{\gamma}^{\beta}$ 's is $\left\langle\left(\mathscr{S}_{\gamma}, \xi_{\gamma}^{i}\right)\right| p(\beta) \leq \gamma<\beta-1$ and $i \in \omega\rangle$, where $\mathscr{S}_{\gamma}=\sigma_{\beta-1}^{\beta}\left(\mathscr{R}_{\gamma}\right)$ and $\xi_{\gamma}^{i}=\sigma_{\beta-1}^{\beta}\left(\eta_{\gamma}^{i+1}\right)$. 
(iv) Let $\delta=\mathrm{T}-\operatorname{pred}(\beta)$, so that by $(1)\left(\right.$ iv) of Definition $4.11, \mathscr{P}_{\beta}^{\beta}=$ $\operatorname{Ult}\left(\mathscr{P}_{\delta}^{\beta}, \pi_{\beta-1}^{\beta}\left(E_{\beta-1}^{\mathscr{T}}\right)\right)$. We define

$$
Q_{\beta}^{\beta}=\operatorname{Ult}\left(Q_{\delta}^{\beta}, \quad \sigma_{\beta-1}^{\beta}\left(\pi_{\beta-1}^{\beta}\left(E_{\beta-1}^{\mathscr{T}}\right)\right)\right),
$$

and let $k: Q_{\delta}^{\beta} \rightarrow Q_{\beta}^{\beta}$ be the canonical embedding so that $k=k_{\delta \beta}$ if $\delta$ survives at $\beta$. Notice that $\sigma_{\delta}^{\beta} \circ \pi_{\delta}^{\beta}$ agrees with $\sigma_{\beta-1}^{\beta} \circ \pi_{\beta-1}^{\beta}$ through $\mu^{\mathscr{T}}(\delta, \beta-1)$ so that the ultrapower yielding $Q_{\beta}^{\beta}$ makes sense. [Let us check the agreement. By $(0)$ of Definition $4.11, \pi_{\delta}^{\beta}$ agrees with $\pi_{\beta-1}^{\beta}$ through $\mu^{\mathscr{T}}(\delta, \beta-1)$. If $p(\beta) \leq \delta<$ $\beta-1$, then $\sigma_{\delta}^{\beta}=\sigma_{\beta-1}^{\beta}\left\lceil\mathscr{P}_{\delta}^{\beta}\right.$, so the desired agreement exists. If $\delta<p(\beta)$, then $\sigma_{\delta}^{\beta}=\sigma_{\delta}^{\beta-1}$, which agrees with $\sigma_{\beta-1}^{\beta-1}$ through $\pi_{\beta-1}^{\beta-1}\left(\mu^{\mathscr{T}}(\delta, \beta-1)\right)$. But $\sigma_{\beta-1}^{\beta-1}$ agrees with $\sigma_{\beta-1}^{\beta}$ through $\rho=\pi_{\beta-1}^{\beta-1}\left(\mu^{\mathscr{T}}(p(\beta)-1, \beta-1)\right) \geq \pi_{\beta-1}^{\beta-1}\left(\mu^{\mathscr{T}}(\delta, \beta-\right.$ $1))$. So $\sigma_{\delta}^{\beta}$ agrees with $\sigma_{\beta-1}^{\beta}$ sufficiently that the desired agreement exists.]

We claim that $Q_{\beta}^{\beta}$ is wellfounded. If $\delta=\beta-1$, this is clear. If $p(\beta) \leq \delta<$ $\beta-1$, then $Q_{\delta}^{\beta} \in Q_{\beta-1}^{\beta}$, and since $Q_{\beta-1}^{\beta}$ satisfies “ $\sigma_{\beta-1}^{\beta}\left(\pi_{\beta-1}^{\beta}\left(E_{\beta-1}^{\mathcal{G}}\right)\right)$ is an extender", this is again clear. If $\delta<p(\beta)$, then $Q_{\delta}^{\beta}=Q_{\delta}^{\beta-1}$. Now $\beta-1<\theta$, and $\theta$ was least such that the sublemma is false for a connected sequence of length $\theta+1$. By induction hypothesis (2), $\left\langle\left(\sigma_{\alpha}^{\gamma} \circ \pi_{\alpha}^{\gamma}, Q_{\alpha}^{\gamma}, \sigma_{\alpha}^{\gamma}\left(\nu_{\alpha}^{\gamma}\right)\right)\right| \alpha \leq \gamma \leq$ $\beta-1\rangle$ is connected. Therefore, Ult $\left(Q_{\delta}^{\beta-1}, \sigma_{\beta-1}^{\beta-1} \pi_{\beta-1}^{\beta-1}\left(E_{\beta-1}^{\mathscr{T}}\right)\right)$ is wellfounded. If $p(\beta)=\beta$, this ultrapower is equal to $\operatorname{Ult}\left(Q_{\delta}^{\beta}, \sigma_{\beta-1}^{\beta}\left(\pi_{\beta-1}^{\beta}\left(E_{\beta-1}^{\mathscr{T}}\right)\right)\right)$ and we are done. So we may assume that $p(\beta)<\beta$. But $\sigma_{\beta-1}^{\beta-1} \circ \pi_{\beta-1}^{\beta-1}=\tau \circ \sigma_{\beta-1}^{\beta} \circ \pi_{\beta-1}^{\beta}$, and $\operatorname{crit}(\tau)>\sigma_{\beta-1}^{\beta} \circ \pi_{\beta-1}^{\beta}\left(\operatorname{crit}\left(E_{\beta-1}^{\mathscr{T}}\right)\right)$. Thus there is a natural embedding $\psi$ from $\operatorname{Ult}\left(Q_{\delta}^{\beta-1}, \sigma_{\beta-1}^{\beta}\left(\pi_{\beta-1}^{\beta}\left(E_{\beta-1}^{\mathscr{T}}\right)\right)\right)$ into $\operatorname{Ult}\left(Q_{\delta}^{\beta-1}, \sigma_{\beta-1}^{\beta-1}\left(\pi_{\beta-1}^{\beta-1}\left(E_{\beta-1}^{\mathscr{T}}\right)\right)\right)$, given by $\psi([a, f])=[\tau(a), f]$. (This is actually a special case of the shift lemma.) It follows that $Q_{\beta}^{\beta}$ is wellfounded in the case $\delta<p(\beta)$ as well.

Let $\mu_{\beta}^{\beta}=k\left(\mu_{\delta}^{\beta}\right)$. From the shift lemma we have an embedding $\sigma$ such that the diagram

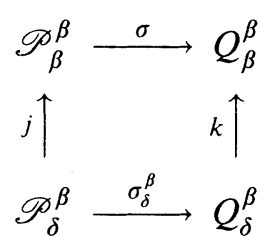

commutes, where $j$ is the canonical embedding (so that $j=j_{\delta \beta}$ if $\delta$ survives at $\beta)$. Now $\sigma$ agrees with $\sigma_{\beta-1}^{\beta}$ through $\pi_{\beta-1}^{\beta}\left(\rho^{\mathscr{T}}(\beta-1, \beta)\right)$. Let $\bar{\eta}=g(\beta-1)$; then $\bar{\eta} \leq \rho^{\mathscr{T}}(\beta-1, \beta)$ as $\mathscr{T}$ is a plus one tree, so $\sigma_{\beta-1}^{\beta}$ agrees with $\sigma$ through $\eta^{*}=\pi_{\beta-1}^{\beta}(\bar{\eta})$. But $\sigma_{\beta-1}^{\beta} \in Q_{\beta-1}^{\beta}$, and $\sigma_{\beta-1}^{\beta} \uparrow V_{\eta *}^{\mathscr{P}_{\beta-1}^{\beta}}$ can be coded by a member of $V_{\eta}^{Q_{\beta-1}^{\beta}}$, where $\eta=\sigma_{\beta-1}^{\beta}\left(\eta^{*}\right)$, as $\eta$ is a successor ordinal. As $Q_{\beta}^{\beta}$ agrees with $Q_{\beta-1}^{\beta}$ through $\eta, \sigma \uparrow V_{\eta *}^{\mathscr{P}_{\beta}^{\beta}} \in Q_{\beta}^{\beta}$. 
We can thus form a tree $\mathscr{U}$ (set of finite sequences closed under initial segment) in $Q_{\beta}^{\beta}$ whose infinite branches are precisely those embeddings $\psi$ : $\mathscr{P}_{\beta}^{\beta} \rightarrow V_{\mu}^{Q_{\beta}^{\beta}}$, where $\mu=\mu_{\beta}^{\beta}$, such that $\psi\left|V_{\eta^{*}}^{\mathscr{P}_{\beta}^{\beta}}=\sigma\right| V_{\eta^{*}}^{\mathscr{P}_{\beta}^{\beta}}$ and $\psi(x)=\sigma(x)$ for those finitely many $x \in \mathscr{P}_{\beta}^{\beta}$ such that $x=j_{\alpha, \beta}(y)$ for some $\alpha$ surviving at $\beta$ with $(\alpha, y)=m(e)$ for some $e \leq n(\beta)$. $\mathscr{U}$ has an infinite branch, namely $\sigma$. We let

$$
\sigma_{\beta}^{\beta}=\text { some infinite branch of } \mathscr{U} \text { belonging to } Q_{\beta}^{\beta} \text {. }
$$

This completes the construction at successor steps. Our induction hypotheses (1)-(5) can be checked routinely.

$\beta$ limit. Set

$$
Q_{\beta}^{\beta}=\text { direct limit of } Q_{\alpha}^{\alpha}, \alpha \text { surviving at } \beta .
$$

Let $k_{\alpha \beta}: Q_{\alpha}^{\alpha} \rightarrow Q_{\beta}^{\beta}$, for $\alpha$ surviving at $\beta$, be the canonical embedding. Notice that $Q_{\beta}^{\beta}$ is wellfounded as it is the direct limit of an internal iteration; cf. remark 5 following Definition 4.11. Set

$$
\mu_{\beta}^{\beta}=\text { common value of } k_{\alpha \beta}\left(\mu_{\alpha}^{\alpha}\right) \text {, for } \alpha \text { surviving at } \beta \text {. }
$$

By our induction hypothesis (3), commutativity at small indices, we have an embedding $\sigma: \mathscr{P}_{\beta}^{\beta} \rightarrow\left(V_{\mu_{\beta}^{\beta}}^{Q_{\beta}^{\beta}}, \in, \delta^{Q_{\beta}^{\beta}}\right)$ given by

$$
\sigma(x)=\text { common value of } k_{\alpha \beta}\left(\sigma_{\alpha}^{\alpha}(y)\right),
$$

where $j_{\alpha, \beta}(y)=x$ and $\alpha$ is sufficiently large.

Now let $\gamma=p(\beta)-1$. For $\alpha \leq \gamma$, we set

$$
\left(\sigma_{\alpha}^{\beta}, Q_{\alpha}^{\beta}, \mu_{\alpha}^{\beta}\right)=\text { common value of }\left(\sigma_{\alpha}^{\delta}, Q_{\alpha}^{\delta}, \mu_{\alpha}^{\delta}\right),
$$

for $\delta<\beta$ sufficiently large.

The existence of such a common value follows from connectedness and the fact that $\liminf _{\xi \rightarrow \beta} p(\xi)=\beta$.

Let $\rho=\pi_{\gamma}^{\beta}\left(\mu^{\mathscr{G}}(\gamma, \beta)\right)=\pi_{\beta}^{\beta}\left(\mu^{\mathscr{T}}(\gamma, \beta)\right)$. We claim that $\sigma$ agrees with $\sigma_{\gamma}^{\beta}$ through $\rho$. To see this, let $\delta \mathrm{T} \beta$ be large enough that $\delta$ survives at $\beta$ and $p(\alpha)>\gamma$ whenever $\delta \leq \alpha<\beta$. We also choose $\delta$ large enough that $\operatorname{crit}\left(j_{\delta \beta}\right)>\rho$ and $\operatorname{crit}\left(k_{\delta \beta}\right)>\sigma_{\gamma}^{\delta}(\rho)$. [For $\gamma<\eta<\beta, \mu^{\mathscr{T}}(\gamma, \beta) \leq$ $g(\eta)<\rho^{\mathscr{G}}(\eta, \eta+1)$. So since $\mathscr{M}_{\beta}^{\mathscr{T}}$ is wellfounded, $\mu^{\mathscr{T}}(\gamma, \beta)<\operatorname{crit}\left(E_{\eta}^{\mathscr{T}}\right)$ for $\eta+1 \in[0, \beta)_{\mathrm{T}}$ sufficiently large. But for such $\eta$ sufficiently large, $\pi_{\eta}^{\eta+1}$ agrees with $\pi_{\gamma}^{\eta+1}$ through $\mu^{\mathscr{T}}(\gamma, \eta), \mu^{\mathscr{T}}(\gamma, \eta) \geq \mu^{\mathscr{T}}(\gamma, \beta)$, and $\pi_{\gamma}^{\eta+1}=\pi_{\gamma}^{\beta}$. Thus $\operatorname{crit}\left(\pi_{\eta}^{\eta+1}\left(E_{\eta}^{\mathcal{G}}\right)\right)>\rho=\pi_{\gamma}^{\beta}\left(\mu^{\mathscr{G}}(\gamma, \beta)\right)$. Thus $\operatorname{crit}\left(j_{\delta \beta}\right)>\rho$ for $\delta \in[0, \beta)_{\mathrm{T}}$ sufficiently large. Similarly, as $\sigma_{\eta}^{\eta+1} \circ \pi_{\eta}^{\eta+1}$ agrees with $\sigma_{\gamma}^{\eta+1} \circ \pi_{\gamma}^{\eta+1}$ through $\mu^{\mathscr{G}}(\gamma, \eta) \geq \mu^{\mathscr{G}}(\gamma, \beta), \operatorname{crit}\left(\sigma_{\eta}^{\eta+1} \circ \pi_{\eta}^{\eta+1}\left(E_{\eta}^{\mathscr{G}}\right)\right)>\sigma_{\gamma}^{\eta+1}(\rho)=\sigma_{\gamma}^{\beta}(\rho)$, for $\eta+1 \in$ 
$[0, \beta)_{\mathrm{T}}$ sufficiently large. So we can choose $\delta$ so that $\operatorname{crit}\left(k_{\delta \beta}\right)>\sigma_{\gamma}^{\delta}(\rho)$. ] It now follows from our induction hypothesis (4), commutativity at small ranks, that $\sigma$ agrees with $\sigma_{\delta}^{\delta}$ through $\rho$. But $\sigma_{\delta}^{\delta}$ agrees with $\sigma_{\gamma}^{\delta}=\sigma_{\gamma}^{\beta}$ through $\rho$. This proves our claim.

Notice also that if $\delta$ is as above, $\sigma_{\delta}^{\delta} \uparrow V_{\rho}^{\mathscr{P}_{\delta}^{\delta}} \in Q_{\delta}^{\delta}$, and $\sigma_{\delta}^{\delta}$ agrees with $\sigma$ through $\rho$. Since $\operatorname{crit}\left(k_{\delta \beta}\right)>\sigma_{\gamma}^{\delta}(\rho)=\sigma_{\delta}^{\delta}(\rho), \sigma \mid V_{\rho}^{\mathscr{P}_{\beta}^{\beta}} \in Q_{\beta}^{\beta}$.

Now let $\left\langle\left(\mathscr{R}_{\alpha}, \eta_{\alpha}^{i}\right) \mid \gamma<\alpha<\beta \wedge i \in \omega\right\rangle$ be in $\mathscr{P}_{\beta}^{\beta}$ and witness (2)(iv) of Definition 4.11. Set

$$
\begin{aligned}
& \mathscr{S}_{\alpha}=\sigma\left(\mathscr{R}_{\alpha}\right), \\
& \mu_{\alpha}^{\beta}=\sigma\left(\eta_{\alpha}^{0}\right), \\
& \xi_{\alpha}^{i}=\sigma\left(\eta_{\alpha}^{i+1}\right),
\end{aligned}
$$

for $\gamma<\alpha<\beta$. So $\left\langle\left(\mathscr{S}_{\alpha}, \xi_{\alpha}^{i}\right) \mid \gamma<\alpha<\beta \wedge i \in \omega\right\rangle$ is in $Q_{\beta}^{\beta}$. Let

$$
Q_{\alpha}^{\beta}=\left(V_{\xi_{\alpha}^{0}}^{\mathscr{S}_{\alpha}}, \in, \delta^{\mathscr{S}_{\alpha}}\right) \text {. }
$$

If we were to let $\sigma_{\alpha}^{\beta}=\sigma \mid \mathscr{P}_{\alpha}^{\beta}$ for $\gamma<\alpha \leq \beta$, all our induction hypotheses would hold, except that possibly $\sigma \notin Q_{\beta}^{\beta}$. However, our standard absoluteness argument will remedy this. There is a tree (set of finite sequences) $\mathscr{U} \in Q_{\beta}^{\beta}$ whose infinite branches are precisely those $\tau: \mathscr{P}_{\beta}^{\beta} \rightarrow\left(V_{\mu_{\beta}^{\beta}}^{Q_{\beta}^{\beta}}, \in, \delta^{Q_{\beta}^{\beta}}\right)$ such that $\tau$ agrees with $\sigma$ through $\rho$ and the restriction of $\tau$ to $\mathscr{P}_{\alpha}^{\beta}$ maps $\mathscr{P}_{\alpha}^{\beta}$ elementarily into $\left(V_{\mu_{\alpha}^{\beta}}^{Q_{\alpha}^{\beta}}, \in, \delta^{Q_{\alpha}^{\beta}}\right)$, and $\tau(x)=\sigma(x)$ for those finitely many $x$ such that $x=j_{\alpha \beta}(y)$ for some $\alpha$ surviving at $\beta$ with $(\alpha, y)=m(e)$ for some $e \leq n(\beta)$. Since $\sigma$ is a branch of $\mathscr{U}$, there is a branch $\tau$ of $\mathscr{U}$ such that $\tau \in Q_{\beta}^{\beta}$. Set

for $\gamma<\alpha \leq \beta$.

$$
\sigma_{\alpha}^{\beta}=\tau \mid Q_{\alpha}^{\beta}
$$

We leave it to the reader to check that induction hypotheses (1)-(5) still hold.

This completes the proof of Sublemma 4.12.

We proceed to the construction.

Sublemma 4.13. There is a connected sequence $\left\langle\mathscr{E}^{\alpha} \mid \alpha<\ln (\mathscr{T})\right\rangle$ of enlargements of $(\mathscr{T}, \mathscr{M})$.

Proof. Let $\left\langle\theta_{\alpha} \mid \alpha<\operatorname{lh}(\mathscr{T})\right\rangle$ witness the $p$-continuous illfoundedness of $(\mathscr{T}$, $\mathscr{M})$. Fix maps $n: \operatorname{lh}(\mathscr{T}) \stackrel{1-1}{\longrightarrow} \omega$ and $m: \omega \stackrel{\text { onto }}{\longrightarrow}\left\{(\beta, x) \mid x \in \mathscr{M}_{\beta}^{\mathscr{T}}\right\}$. We shall define $\mathscr{E}^{\alpha}=\left\langle\left(\pi_{\beta}^{\alpha}, \mathscr{P}_{\beta}^{\alpha}, \nu_{\beta}^{\alpha}\right) \mid \beta \leq \alpha\right\rangle$ by induction on $\alpha<\operatorname{lh}(\mathscr{T})$. We maintain inductively, in addition to connectedness:

(1) $\pi_{\beta}^{\alpha} \in \mathscr{P}_{\beta}^{\alpha}$ for all $\beta \leq \alpha$.

(2) $V_{\omega+1} \subseteq \mathscr{P}_{\beta}^{\alpha}$ for all $\beta \leq \alpha$. 
For $\beta \leq \alpha$, let $c(\beta, \alpha)=\operatorname{card}(\{\gamma>\alpha \mid p(\gamma) \leq \beta\})$. Since $\liminf _{\gamma \rightarrow \lambda} p(\gamma)=$ $\lambda$ for all limit $\lambda, c(\beta, \alpha)<\omega$ for all $\beta \leq \alpha$.

(3) (Enough room to take hulls) If $\beta<\alpha$, then the set of ordinals $\eta \in \mathscr{P}_{\beta}^{\alpha}$ such that $\eta>\nu_{\beta}^{\alpha}$ and $\left(V_{\eta}^{\mathscr{P}_{\beta}^{\alpha}}, \in, \delta^{\mathscr{P}_{\beta}^{\alpha}}\right)$ is a premouse has order type at least $\omega^{2} \cdot \pi_{\beta}^{\alpha}\left(\theta_{\beta}\right)+\omega \cdot c(\beta, \alpha)+1$. If $\beta=\alpha$, the set of such ordinals $\eta$ has order type at least $\omega^{2} \cdot \pi_{\alpha}^{\alpha}\left(\theta_{\alpha}\right)+\omega^{2}$.

(4) (Commutativity at small indices) Suppose $m(e)=(\beta, x)$ where $\beta$ survives at $\alpha$, and suppose $\gamma \in[\beta, \alpha)_{\mathrm{T}}$ is such that $e \leq n(\xi)$ for all $\xi \in$ $(\gamma, \alpha]_{\mathrm{T}}$. Then letting $y=i_{\beta \gamma}^{\mathscr{G}}(x)$, we have $j_{\gamma \alpha}\left(\pi_{\gamma}^{\gamma}(y)\right)=\pi_{\alpha}^{\alpha}\left(i_{\gamma \alpha}^{\mathscr{T}}(y)\right)$.

(5) (Commutativity at small ranks) Suppose $\beta$ survives at $\alpha$, and let $\delta$ be small enough that $\delta<p(\gamma)$ whenever $\beta<\gamma \leq \alpha$. Let $\rho=\mu^{\mathscr{T}}(\delta, \alpha)$ and $x \in V_{\rho}^{\mathscr{M}_{\beta}^{\mathscr{G}}} \cup\{\rho\}$, and suppose $\operatorname{crit}\left(i_{\beta \alpha}^{\mathscr{T}}\right)>\rho$. Then $j_{\beta \alpha}\left(\pi_{\beta}^{\beta}(x)\right)=\pi_{\alpha}^{\alpha}\left(i_{\beta \alpha}^{\mathscr{G}}(x)\right)$.

We now define the $\mathscr{E}^{\alpha}$ 's

$\mathscr{E}^{0}$. Recall that $\pi: \mathscr{M} \rightarrow\left(V_{\gamma_{0}}, \in, \delta_{0}\right)$ elementarily. Let $\beta$ be such that $\left(V_{\beta}, \varepsilon, \delta_{0}\right)$ is a premouse and there are in order type $\omega^{2} \cdot \pi\left(\theta_{0}\right)+\omega^{2}$ many ordinals $\eta$ such that $\gamma_{0}<\eta<\beta$ and $\left(V_{\eta}, \in, \delta_{0}\right)$ is a premouse. Set

$$
\begin{aligned}
\mathscr{P}_{0}^{0} & =\left(V_{\beta}, \in, \delta_{0}\right), \\
\nu_{0}^{0} & =\gamma_{0}, \\
\pi_{0}^{0} & =\pi .
\end{aligned}
$$

$\mathscr{E}^{\alpha+1}$. Let $p(\alpha+1)=\beta+1$.

(i) Let $\mathscr{E}_{\gamma}^{\alpha+1}=\mathscr{E}_{\gamma}^{\alpha}$ for $\gamma \leq \beta$.

(ii) Suppose $\beta+1<\alpha$. By induction hypothesis (3), given $\gamma$ such that $\beta+$ $1 \leq \gamma<\alpha$, we have that the set of ordinals $\eta \in \mathscr{P}_{\gamma}^{\alpha}$ such that $\left(V_{\eta}^{\mathscr{P}_{\gamma}^{\alpha}}, \in, \delta^{\mathscr{P}_{\gamma}^{\alpha}}\right)$ is a premouse and $\eta>\nu_{\gamma}^{\alpha}$ has order type at least $\omega^{2} \cdot \pi_{\gamma}^{\alpha}\left(\theta_{\gamma}\right)+\omega \cdot c(\gamma, \alpha)+1$. Let $\eta_{\gamma}$ be the $\omega^{2} \cdot \pi_{\gamma}^{\alpha}\left(\theta_{\gamma}\right)+\omega \cdot c(\gamma, \alpha)+1$ st such "premouse ordinal" so that $\eta_{\gamma} \in \mathscr{P}_{\gamma}^{\alpha}$. (Our convention here is that if $\lambda$ is a limit, then $\lambda$ is the $(\lambda+1)$ st ordinal.) Let $\rho_{\gamma}=\pi_{\gamma}^{\alpha}\left(\mu^{\mathscr{T}}(\gamma, \alpha)\right)$, and set

$$
\begin{aligned}
\mathscr{S}_{\gamma}= & \text { transitive collapse of Skolem closure of } \\
& V_{\rho_{\gamma}}^{\mathscr{P}_{\gamma}^{\alpha}} \cup\left\{\rho_{\gamma}, \pi_{\gamma}^{\alpha}, \nu_{\gamma}^{\alpha}\right\} \text { inside }\left(V_{\eta_{\gamma}}^{\mathscr{P}^{\alpha}}, \in, \delta^{\mathscr{P}_{\gamma}^{\alpha}}\right),
\end{aligned}
$$

and

$$
\begin{aligned}
& \mu_{\gamma}=\text { image of } \nu_{\gamma}^{\alpha} \text { under collapse } \\
& \sigma_{\gamma}=\text { image of } \pi_{\gamma}^{\alpha} \text { under collapse. }
\end{aligned}
$$

Notice that $\mathscr{S}_{\gamma} \in \mathscr{P}_{\alpha}^{\alpha}$, since $\mathscr{S}_{\gamma}$ is coded by a member of $V_{\rho_{\gamma}+1}^{\mathscr{P}_{\gamma}^{\alpha}}$, so we can apply induction hypothesis (2).

Now clearly, for $\beta+1 \leq \gamma<\alpha$,

(a) $\mathscr{S}_{\gamma}$ is a premouse, 
(b) $\sigma_{\gamma}: \mathscr{M}_{\gamma}^{\mathscr{T}} \rightarrow\left(V_{\mu_{\gamma}}^{\mathscr{S}_{\gamma}}, \in, \delta^{\mathscr{S}_{\gamma}}\right)$ elementarily, and $\sigma_{\gamma} \in \mathscr{S}_{\gamma}$,

(c) $\sigma_{\gamma}$ agrees with $\pi_{\alpha}^{\alpha}$ through $\mu^{\mathscr{T}}(\gamma, \alpha)$,

(d) $\mathscr{S}_{\gamma} \vDash$ "There are, in order type, $\omega^{2} \cdot \sigma_{\gamma}\left(\theta_{\gamma}\right)+\omega \cdot c(\gamma, \alpha)$ ordinals $\eta>\mu_{\gamma}$ such that $\left(V_{\eta}, \in, \delta^{\mathscr{S}_{\gamma}}\right)$ is a premouse".

There is a tree in $\mathscr{P}^{\alpha}$ whose infinite branches correspond to sequences $\left\langle\left(\mathscr{S}_{\gamma}^{\prime}, \sigma_{\gamma}^{\prime}, \mu_{\gamma}^{\prime}\right) \mid \beta+1 \leq \gamma<\alpha\right\rangle$ having properties (a)-(d). (As to (c), notice that $\pi_{\alpha}^{\alpha} \in \mathscr{P}_{\alpha}^{\alpha}$.) Because $\left(\mathscr{S}_{\gamma}, \sigma_{\gamma}, \mu_{\gamma}\right) \in \mathscr{P}_{\alpha}^{\alpha}$ for all $\gamma$, this tree has an infinite branch and, hence, an infinite branch in $\mathscr{P}_{\alpha}^{\alpha}$. Therefore, we can fix a sequence $\Gamma=\left\langle\left(\mathscr{S}_{\gamma}^{\prime}, \sigma_{\gamma}^{\prime}, \mu_{\gamma}^{\prime}\right) \mid \beta+1 \leq \gamma<\alpha\right\rangle$ belonging to $\mathscr{P}_{\alpha}^{\alpha}$ and having properties (a)-(d). If $\beta+1 \geq \alpha$, we set $\Gamma=\varnothing$.

(iii) Suppose $\beta<\alpha$. Let $\eta$ be the $\omega^{2} \cdot \pi_{\alpha}^{\alpha}\left(\theta_{\alpha}\right)+\omega \cdot c(\alpha, \alpha)+1$ st ordinal $\xi>\nu_{\alpha}^{\alpha}$ such that $\left(V_{\xi}^{\mathscr{P}_{\alpha}^{\alpha}}, \in, \delta^{\mathscr{P}_{\alpha}^{\alpha}}\right)$ is a premouse. Let $\rho=\pi_{\alpha}^{\alpha}\left(\mu^{\mathscr{T}}(\beta, \alpha)\right)=$ $\pi_{\beta}^{\alpha}\left(\mu^{\mathscr{T}}(\beta, \alpha)\right)$. Then we set

$$
\begin{aligned}
\mathscr{P}_{\alpha}^{\alpha+1}= & \text { transitive collapse of Skolem closure of } \\
& V_{\rho+1}^{\mathscr{P}_{\alpha}^{\alpha}} \cup\left\{\Gamma, \pi_{\alpha}^{\alpha}, \nu_{\alpha}^{\alpha}\right\} \text { inside }\left(V_{\eta}^{\mathscr{P}_{\alpha}^{\alpha}}, \in, \delta^{\mathscr{P}_{\alpha}^{\alpha}}\right),
\end{aligned}
$$

and

$$
\left(\pi_{\alpha}^{\alpha+1}, \nu_{\alpha}^{\alpha+1}\right)=\text { image of }\left(\pi_{\alpha}^{\alpha}, \nu_{\alpha}^{\alpha}\right) \text { under collapse. }
$$

If $\beta+1<\alpha$, then for $\beta+1 \leq \gamma<\alpha$ let $\mathscr{R}_{\gamma}=$ image of $\mathscr{S}_{\gamma}^{\prime}$ under collapse, $\pi_{\gamma}^{\alpha+1}=$ image of $\sigma_{\gamma}^{\prime}$ under collapse, and $\nu_{\gamma}^{\alpha+1}=$ image of $\mu_{\gamma}^{\prime}$ under collapse. Let also, for $i \in \omega$,

$$
\begin{aligned}
\eta_{\gamma}^{i}= & \omega^{2} \cdot \pi_{\gamma}^{\alpha+1}\left(\theta_{\gamma}\right)+\omega \cdot c(\gamma, \alpha+1)+(i+2) \text { nd } \\
& \text { ordinal } \xi>\nu_{\gamma}^{\alpha+1} \text { such that }\left(V_{\xi}^{\mathscr{R}_{\gamma}}, \in, \delta^{\mathscr{R}_{\gamma}}\right) \text { is a premouse. }
\end{aligned}
$$

(Notice that $c(\gamma, \alpha+1)=c(\gamma, \alpha)-1$ when $p(\alpha+1) \leq \gamma$, so $\eta_{\gamma}^{i}$ exists.) Set

$$
\mathscr{P}_{\gamma}^{\alpha+1}=\left(V_{\eta_{\gamma}^{0}}^{\mathscr{R}_{\gamma}}, \in, \delta^{\mathscr{R}_{\gamma}}\right)
$$

for $\beta+1 \leq \gamma<\alpha$.

(iv) Finally, let $\delta=\mathrm{T}$-pred $(\alpha+1)$, and set

$$
\mathscr{P}_{\alpha+1}^{\alpha+1}=\operatorname{Ult}\left(\mathscr{P}_{\delta}^{\alpha+1}, \pi_{\alpha}^{\alpha+1}\left(E_{\alpha}^{\mathscr{T}}\right)\right)
$$

We can embed $\mathscr{P}_{\alpha+1}^{\alpha+1}$ into $\operatorname{Ult}\left(\mathscr{P}_{\delta}^{\alpha}, \pi_{\alpha}^{\alpha}\left(E_{\alpha}^{\mathscr{T}}\right)\right)$ if $\delta<p(\alpha+1)$, and the latter is wellfounded by Sublemma 4.12. If $p(\alpha+1) \leq \delta$, then $\mathscr{P}_{\delta}^{\alpha+1} \in \mathscr{P}_{\alpha}^{\alpha+1}$ or $\mathscr{P}_{\delta}^{\alpha+1}=\mathscr{P}_{\alpha}^{\alpha+1}$, so $\mathscr{P}_{\alpha+1}^{\alpha+1}$ is wellfounded because it results from an internal ultrapower. So $\mathscr{P}_{\alpha+1}^{\alpha+1}$ is wellfounded. Let $j: \mathscr{P}_{\delta}^{\alpha+1} \rightarrow \mathscr{P}_{\alpha+1}^{\alpha+1}$ be the canonical embedding, and $j_{\delta, \alpha+1}=j$ if $\delta$ survives at $\alpha+1$. Set $\nu_{\alpha+1}^{\alpha+1}=j\left(\nu_{\delta}^{\alpha+1}\right)$. 
Let $\sigma: \mathscr{M}_{\alpha+1}^{\mathscr{I}} \rightarrow\left(V_{\nu}^{\mathscr{P}_{\alpha+1}^{\alpha+1}}, \in, \delta^{\mathscr{P}_{\alpha+1}^{\alpha+1}}\right)$, where $\nu=\nu_{\alpha+1}^{\alpha+1}$, be given by the shift lemma. By a now familiar argument we can find $\pi \in \mathscr{P}_{\alpha+1}^{\alpha+1}$ such that $\pi$ agrees with $\sigma$ through $\mu^{\mathscr{G}}(\alpha, \alpha+1)$ and $\pi(x)=\sigma(x)$ whenever there is an $e \leq n(\alpha+1)$ such that $m(e)=(\gamma, y)$ for some $\gamma \in[0, \delta]_{\mathrm{T}}$ such that $\gamma$ survives at $\alpha+1$ and $x=i_{\gamma, \alpha+1}^{\mathscr{G}}(y)$. We also choose $\pi$ so that $\pi\left(i_{\delta, \alpha+1}^{\mathscr{g}}\left(\theta_{\delta}\right)\right)=$ $\sigma\left(i_{\delta, \alpha+1}^{\mathscr{G}}\left(\theta_{\delta}\right)\right)$. Set $\pi_{\alpha+1}^{\alpha+1}=\pi$.

This completes the definition of $\mathscr{E}^{\alpha+1}$. One can check the induction hypotheses routinely. We do so for (3), the "enough room" condition.

Let $\gamma \leq \alpha+1$; we must see that the set of $\eta \in \mathscr{P}_{\gamma}^{\alpha+1}$ such that $\eta>\nu_{\gamma}^{\alpha+1}$ and $\left(V_{\eta}^{\mathscr{P}_{\gamma}^{\alpha+1}}, \in, \delta_{\gamma}^{\mathscr{P}_{\gamma}^{\alpha+1}}\right)$ is a premouse has order type at least $\omega^{2} \cdot \pi_{\gamma}^{\alpha+1}\left(\theta_{\gamma}\right)+\omega$. $c(\gamma, \alpha+1)+1$ if $\gamma<\alpha+1$, and at least $\omega^{2} \cdot \pi_{\gamma}^{\alpha+1}\left(\theta_{\gamma}\right)+\omega^{2}$ if $\gamma=\alpha+1$. But now if $\gamma \leq \beta=p(\alpha+1)-1$ then $\mathscr{E}_{\gamma}^{\alpha+1}=\mathscr{E}_{\gamma}^{\alpha}$, so this is immediate by induction. If $\beta<\gamma<\alpha$, this holds because of the construction (the tree which produced $\mathscr{S}_{\gamma}^{\prime}$, and the collapse-producing $\left.\mathscr{R}_{\gamma}\right)$ and the fact that $c(\gamma, \alpha+1)<c(\gamma, \alpha)$. If $\beta<\gamma=\alpha$, it holds by construction and the fact $c(\alpha, \alpha+1)<c(\alpha, \alpha)$. Finally, suppose $\gamma=\alpha+1$.

Case 1. $\delta$ survives at $\alpha+1$. In this case $p(\xi)>\delta$ for $\delta<\xi \leq \alpha+1$, so $\mathscr{E}_{\delta}^{\alpha+1}=$ $\mathscr{E}_{\delta}^{\delta}$. By our induction hypothesis at stage $\delta$, we have $\omega^{2} \cdot \pi_{\delta}^{\delta}\left(\theta_{\delta}\right)+\omega^{2}$ premouse ordinals above $\nu_{\delta}^{\delta}$ in $\mathscr{P}_{\delta}^{\delta}$. Hence we have $\omega^{2} \cdot \pi_{\delta}^{\alpha+1}\left(\theta_{\delta}\right)+\omega^{2}$ premouse ordinals above $\nu_{\delta}^{\alpha+1}$ in $\mathscr{P}_{\delta}^{\alpha+1}$. Recall we enforced $\pi_{\alpha+1}^{\alpha+1}\left(i_{\delta, \alpha+1}^{\mathscr{G}}\left(\theta_{\delta}\right)\right)=\sigma\left(i_{\delta, \alpha+1}^{\mathscr{G}}\left(\theta_{\delta}\right)\right)$, and $j \circ \pi_{\delta}^{\alpha+1}=\sigma \circ i_{\delta, \alpha+1}^{\mathscr{g}}$. Hence we have $\omega^{2} \cdot j_{\delta, \alpha+1}\left(\pi_{\delta}^{\alpha+1}\left(\theta_{\delta}\right)\right)+\omega^{2}=\omega^{2}$. $\pi_{\alpha+1}^{\alpha+1}\left(i_{\delta, \alpha+1}^{\mathscr{G}}\left(\theta_{\delta}\right)\right)+\omega^{2}$ many such ordinals in $\mathscr{P}_{\alpha+1}^{\alpha+1}$ above $\nu_{\alpha+1}^{\alpha+1}=j_{\delta, \alpha+1}\left(\nu_{\delta}^{\alpha+1}\right)$. But $i_{\delta, \alpha+1}^{\mathscr{G}}\left(\theta_{\delta}\right)=\theta_{\alpha+1}$ as $\delta$ survives at $\alpha+1$, so we have $\omega^{2} \cdot \pi_{\alpha+1}^{\alpha+1}\left(\theta_{\alpha+1}\right)+\omega^{2}$ many such ordinals, as desired.

Case 2. $\delta$ does not survive at $\alpha+1$. We have at least $\omega^{2} \cdot \pi_{\delta}^{\alpha+1}\left(\theta_{\delta}\right)$ many premouse ordinals above $\nu_{\delta}^{\alpha+1}$ in $\mathscr{P}_{\delta}^{\alpha+1}$. Hence we have at least $\omega^{2} \cdot j\left(\pi_{\delta}^{\alpha+1}\left(\theta_{\delta}\right)\right)=$ $\omega^{2} \cdot \pi_{\alpha+1}^{\alpha+1}\left(i_{\delta, \alpha+1}^{\mathscr{G}}\left(\theta_{\delta}\right)\right)$ many premouse ordinals above $\nu_{\alpha+1}^{\alpha+1}$ in $\mathscr{P}_{\alpha+1}^{\alpha+1}$. (Recall we enforced $\pi_{\alpha+1}^{\alpha+1}\left(i_{\delta, \alpha+1}^{\mathscr{G}}\left(\theta_{\delta}\right)\right)=\sigma\left({ }_{\delta, \alpha+1}^{\mathscr{g}}\left(\theta_{\delta}\right)\right)$, and $j \circ \pi_{\delta}^{\alpha+1}=\sigma \circ i_{\delta, \alpha+1}^{\mathscr{g}}$. $)$

But $\theta_{\alpha+1}<i_{\delta, \alpha+1}^{\mathscr{g}}\left(\theta_{\delta}\right)$ because $\delta$ does not survive at $\alpha+1$, so there are at least $\omega^{2} \cdot \pi_{\alpha+1}^{\alpha+1}\left(\theta_{\alpha+1}\right)+\omega^{2}$ many such ordinals, as desired.

Thus the enough room condition holds for $\mathscr{E}^{\alpha+1}$. We leave the other conditions to the reader.

$\mathscr{E}^{\alpha}, \alpha$ limit. Let

$$
\mathscr{P}_{\alpha}^{\alpha}=\text { direct limit of } \mathscr{P}_{\beta}^{\beta}, \quad \beta \text { surviving at } \alpha .
$$

As $\mathscr{P}_{\alpha}^{\alpha}$ is the limit of an internal iteration, it is wellfounded. Let $j_{\beta \alpha}$ : $\mathscr{P}_{\beta}^{\beta} \rightarrow \mathscr{P}_{\alpha}^{\alpha}$ be the canonical embedding, for $\beta$ surviving at $\alpha$. Set $\nu_{\alpha}^{\alpha}=$ common value of $j_{\beta \alpha}\left(\nu_{\beta}^{\beta}\right)$, for $\beta$ surviving at $\alpha$. Our induction hypothesis 
(4) gives pointwise eventual commutativity of the $j$ 's and $\pi$ 's along $[0, \alpha)_{\mathrm{T}}$ and, hence, on embedding $\pi: \mathscr{M}_{\alpha}^{\mathscr{T}} \rightarrow\left(V_{\nu}^{\mathscr{P}_{\alpha}^{\alpha}}, \in, \delta^{\mathscr{P}_{\alpha}^{\alpha}}\right)$, where $\nu=\nu_{\alpha}^{\alpha}$, given by

$$
\begin{aligned}
\pi(x)= & \text { common value of } j_{\beta \alpha}\left(\pi_{\beta}^{\beta}(y)\right), \\
& \text { where } i_{\beta \alpha}^{\mathscr{T}}(y)=x \text { and } \beta T \alpha \text { is sufficiently large. }
\end{aligned}
$$

Now let $p(\alpha)=\beta+1$. For $\gamma \leq \beta$ we set $\mathscr{E}_{\gamma}^{\alpha}=$ eventual value of $\mathscr{C}_{\gamma}^{\delta}$, for $\delta<\alpha$ sufficiently large. For $\beta<\gamma<\alpha$, let $\left(\tau_{\gamma}, Q_{\gamma}, \mu_{\gamma}\right)$ be the eventual value of $\left(\pi_{\gamma}^{\delta}, \mathscr{P}_{\gamma}^{\delta}, \nu_{\gamma}^{\delta}\right)$, for $\delta<\alpha$ sufficiently large. Let also

$$
\begin{aligned}
& \mathscr{S}=\text { transitive collapse of Skolem closure of } \\
& \qquad V_{\rho}^{Q_{\gamma}} \cup\left\{\rho, \tau_{\gamma}, \mu_{\gamma}\right\} \text { inside }\left(V_{\eta}^{Q_{\gamma}}, \in, \delta^{Q_{\delta}}\right),
\end{aligned}
$$

where $\rho=\tau_{\gamma}\left(\mu^{\mathscr{T}}(\gamma, \alpha)\right)$ and $\eta$ is the $\omega^{2} \cdot \tau_{\gamma}\left(\theta_{\gamma}\right)+\omega \cdot(c(\gamma, \alpha)+1)+1$ st premouse ordinal in $Q_{\gamma}$ above $\mu_{\gamma}$. (Notice $c(\gamma, \alpha)<c(\gamma, \delta$ ) for $\delta<\alpha$ sufficiently large.) Let

$$
\left(\sigma_{\gamma}, \nu_{\gamma}\right)=\text { image of }\left(\tau_{\gamma}, \mu_{\gamma}\right) \text { under collapse. }
$$

Fix for a moment $\gamma$ such that $\beta<\gamma<\alpha$. Let $\rho=\sigma_{\gamma}\left(\mu^{\mathscr{T}}(\gamma, \alpha)\right)=$ eventual value of $\pi_{\gamma}^{\delta}\left(\mu^{\mathscr{T}}(\gamma, \alpha)\right), \delta<\alpha$ sufficiently large. Since $\mathscr{P}_{\alpha}^{\alpha}$ is wellfounded, $\operatorname{crit}\left(j_{\delta \alpha}\right)>\rho$ for $\delta$ sufficiently large surviving at $\alpha$. So by remark (3) following Definition 4.11, $V_{\rho+1}^{Q_{\gamma}} \subseteq \mathscr{P}_{\alpha}^{\alpha}$, and therefore $\mathscr{S}_{\gamma} \in \mathscr{P}_{\alpha}^{\alpha}$. We also have

(a) $\mathscr{S}_{\gamma}$ is a premouse,

(b) $\sigma_{\gamma}: \mathscr{M}_{\gamma}^{\mathscr{T}} \rightarrow\left(V_{\nu_{\delta}}^{\mathscr{S}_{\gamma}}, \in, \delta^{\mathscr{S}_{\gamma}}\right)$ elementarily, and $\sigma_{\gamma} \in \mathscr{S}_{\gamma}$,

(c) $\sigma_{\gamma}$ agrees with $\pi$ through $\rho=\sigma_{\gamma}\left(\mu^{\mathscr{T}}(\gamma, \alpha)\right)$,

(d) $\mathscr{S}_{\gamma} \vDash$ "There are, in order type, $\omega^{2} \cdot \sigma_{\gamma}\left(\theta_{\gamma}\right)+\omega \cdot(c(\gamma, \alpha)+1)$ premouse ordinals $\eta>\nu_{\gamma}$ ",

(e) $\pi$ agrees with $\pi_{\beta}^{\alpha}$ through $\mu^{\mathscr{T}}(\beta, \alpha)$.

Now $\pi_{\beta}^{\alpha} \uparrow V_{\xi}^{\mathscr{M}_{\beta}^{\mathscr{g}}} \in \mathscr{P}_{\alpha}^{\alpha}$, where $\xi=\mu^{\mathscr{T}}(\beta, \alpha)$. It follows that there is a tree $\mathscr{U} \in \mathscr{P}_{\alpha}^{\alpha}$ whose infinite branches correspond to sequences $\left\langle\left(\mathscr{S}_{\gamma}^{\prime}, \sigma_{\gamma}^{\prime}, \mu_{\gamma}^{\prime}\right)\right.$ | $\beta<\gamma<\alpha\rangle$ together with embeddings $\pi^{\prime}: \mathscr{M}_{\alpha}^{\mathscr{T}} \rightarrow\left(V_{\nu_{\alpha}^{\alpha}}^{\mathscr{P}^{\alpha}}, \in, \delta^{\mathscr{P}_{\alpha}^{\alpha}}\right)$ having properties $(\mathrm{a})-(\mathrm{e})$ of $\left\langle\left(\mathscr{S}_{\gamma}, \sigma_{\gamma}, \mu_{\gamma}\right) \mid \beta<\gamma<\alpha\right\rangle$ and $\pi$. We can arrange that $\mathscr{U}$ also forces $\pi^{\prime}\left(\theta_{\alpha}\right)=\pi\left(\theta_{\alpha}\right)$ and $\pi^{\prime}(x)=\pi(x)$ for those finitely many $x$ such that $x=(m(e))_{1}$, for some $e \leq n(\alpha)$. $\mathscr{U}$ has a branch, hence a branch in $\mathscr{P}_{\alpha}^{\alpha}$. So we fix $\left\langle\left(\mathscr{S}_{\gamma}^{\prime}, \sigma_{\gamma}^{\prime}, \mu_{\gamma}^{\prime}\right) \mid \beta<\gamma<\alpha\right\rangle$ and $\pi^{\prime}$ which belong to $\mathscr{P}_{\alpha}^{\alpha}$ and have properties (a)-(e). Set $\pi_{\alpha}^{\alpha}=\pi^{\prime}$, and for $\beta<\gamma<\alpha$, let $\eta_{\gamma}^{i}=\omega^{2} \cdot \sigma_{\gamma}^{\prime}\left(\theta_{\gamma}\right)+\omega \cdot c(\gamma, \alpha)+(i+2)$ nd premouse ordinal in $\mathscr{S}_{\gamma}^{\prime}$ above $\nu_{\gamma}^{\prime}$, 


$$
\begin{aligned}
& \mathscr{P}_{\gamma}^{\alpha}=\left(V_{\eta_{\gamma}^{0}}^{\mathscr{S}_{\gamma}^{\prime}}, \in, \delta^{\mathscr{S}_{\gamma}^{\prime}}\right), \\
& \nu_{\gamma}^{\alpha}=\mu_{\gamma}^{\prime}, \\
& \pi_{\gamma}^{\alpha}=\sigma_{\gamma}^{\prime} .
\end{aligned}
$$

It is easy to check the induction hypotheses (1)-(5) for $\left\langle\mathscr{E}^{\beta} \mid \beta \leq \alpha\right\rangle$.

This completes the proof of Sublemma 4.13.

We now complete the proof of Lemma 4.6. Suppose first that $\operatorname{lh}(\mathscr{T})=\gamma+1$ and $E \in \mathscr{M}_{\gamma}^{\mathscr{T}}$ is the extender we fixed for the purpose of defining $\mu^{\mathscr{T}}(\alpha, \delta)$ for $\alpha<\delta \leq \gamma$. So $\operatorname{Ult}\left(\mathscr{M}_{\beta}^{\mathscr{T}}, E\right)$ is illfounded and $\operatorname{crit}(E)<\mu^{\mathscr{T}}(\beta, \gamma)$. Let $\left\langle\mathscr{E}^{\alpha} \mid \alpha \leq \gamma\right\rangle$ be a connected sequence of enlargements of $(\mathscr{T}, \mathscr{M})$. By Sublemma 4.12, Ult $\left(\mathscr{P}_{\beta}^{\gamma}, \pi_{\gamma}^{\gamma}(E)\right)$ is wellfounded. By the shift lemma, $\operatorname{Ult}\left(\mathscr{M}_{\beta}^{\mathscr{T}}, E\right)$ embeds into $\operatorname{Ult}\left(\mathscr{P}_{\beta}^{\gamma}, \pi_{\gamma}^{\gamma}(E)\right)$. This is a contradiction.

Suppose next $\ln (\mathscr{T})$ is a limit ordinal. Recall that there is a strictly increasing sequence $\left\langle\alpha_{i} \mid i \in \omega\right\rangle$ such that $\sup _{i} \alpha_{i}=\lambda$ and $\forall i\left[p\left(\alpha_{i+1}+1\right)=\alpha_{i}+1\right.$ and $\left.\forall \eta\left(\alpha_{i}+1<\eta \leq \alpha_{i+1} \Rightarrow \alpha_{i}+1<p(\eta)\right)\right]$. Again, let $\left\langle\mathscr{E}^{\alpha} \mid \alpha<\operatorname{lh}(\mathscr{T})\right\rangle$ be a connected sequence of enlargements of $(\mathscr{T}, \mathscr{M})$. We claim that for $i \geq 1$

$$
\mathscr{P}_{\alpha_{i}}^{\alpha_{i}+1} \in \mathscr{P}_{\alpha_{i-1}}^{\alpha_{i-1}+1}
$$

a contradiction. For set $\beta=\alpha_{i-1}$ and $\alpha=\alpha_{i}$. We have $p(\alpha+1)=\beta+1 \leq$ $\alpha$, so (1) (ii) of Definition 4.11 implies that $\mathscr{P}_{\alpha}^{\alpha+1}$ is coded by a member of $V_{\rho+2}^{\mathscr{P}_{\alpha}^{\alpha}}$, where $\rho=\pi_{\alpha}^{\alpha}\left(\mu^{\mathscr{T}}(\beta, \alpha)\right)=\pi_{\beta}^{\alpha}\left(\mu^{\mathscr{T}}(\beta, \alpha)\right)$. We can now apply remark 3 following Definition 4.11, noting that $\rho \leq \pi_{\alpha}^{\alpha}\left(\mu^{\mathscr{T}}(\beta+1, \alpha)\right)$ and $\forall \eta(\beta+1<\eta \leq \alpha \Rightarrow \beta+1<p(\eta))$, to conclude that $V_{\rho+2}^{\mathscr{P}_{\alpha}^{\alpha}} \subseteq \mathscr{P}_{\beta+1}^{\beta+1}$. Thus it is enough to show that $V_{\rho+2}^{\mathscr{P}_{\beta+1}^{\beta+1}} \subseteq \mathscr{P}_{\beta}^{\beta+1}$; then $\mathscr{P}_{\alpha}^{\alpha+1} \in \mathscr{P}_{\beta}^{\beta+1}$. But $\mathscr{P}_{\beta+1}^{\beta+1}=$ $\operatorname{Ult}\left(\mathscr{P}_{\delta}^{\beta}, \pi_{\beta}^{\beta+1}\left(E_{\beta}^{\mathscr{T}}\right)\right)$, where $\delta=\mathrm{T}$-pred $(\beta+1)$, so $\mathscr{P}_{\beta+1}^{\beta+1}$ agrees with $\mathscr{P}_{\beta}^{\beta+1}$ through $\gamma=\pi_{\beta}^{\beta+1}\left(\rho^{\mathscr{T}}(\beta, \beta+1)\right)$, and $V_{\gamma+1}^{\mathscr{P}_{\beta+1}^{\beta+1}} \subseteq V_{\gamma+1}^{\mathscr{P}_{\beta}^{\beta+1}}$. Now $\mu^{\mathscr{T}}(\beta, \alpha) \leq$ $g(\beta)=1$ st successor after $\mu^{\mathscr{T}}(\beta)<\rho^{\mathscr{T}}(\beta, \beta+1)$, as $\mathscr{T}$ is a plus two tree. Since $\beta+1<p(\eta)$ for all $\eta$ such that $\beta+1<\eta \leq \alpha, \pi_{\beta}^{\beta+1}=\pi_{\beta}^{\alpha}$. So $\mathscr{P}_{\beta+1}^{\beta+1}$ agrees with $\mathscr{P}_{\beta}^{\beta+1}$ through $\gamma=\pi_{\beta}^{\alpha}\left(\rho^{\mathscr{T}}(\beta, \beta+1)\right) \geq \rho+1$, and $V_{\gamma+1}^{\mathscr{P}_{\beta+1}^{\beta+1}} \subseteq \mathscr{P}_{\beta}^{\beta+1}$, as desired.

This contradiction completes the proof of Lemma 4.6. That, in turn, completes the proof of Theorem 4.3.

\section{SOME OPEN PROBLEMS}

Many basic questions about iteration trees remain open. In this section we discuss some of them. We shall state our questions in full generality, but we feel free to restrict ourselves to normal iteration trees whenever it helps to do so. 
A. Unique Branches. The Unique Branches Hypothesis, or UBH, is the following.

UBH. Let $\mathscr{T}$ be an iteration tree on $V$ of limit length; then $\mathscr{T}$ has at most one cofinal wellfounded branch.

The results of $\S 2$ might be regarded as a step toward a proof of UBH. Viewed this way, however, they are significantly lacking because they make no use of the assumption that the base model of $\mathscr{T}$ is $V$ (or, what amounts to about the same thing, that $\mathscr{T}$ belongs to its base model). The following two examples show the need for such an assumption, even in the case of the simplest $\mathscr{T}$ for which UBH has content, namely, alternating chains.

An alternating chain is an iteration tree of length $\omega$ having the two cofinal branches $b=\{2 i \mid i \in \omega\}$ and $c=\{0\} \cup\{2 i+1 \mid i \in \omega\}$. (This completely determines the tree order.) We shall assume the reader is familiar with the method for constructing alternating chains of [MS].

The next proposition, or something like it, was discovered independently by H. Woodin.

Proposition 5.1. Let $\delta$ be Woodin; then there is a countable premouse $\mathscr{M}$ and an elementary $\pi: \mathscr{M} \rightarrow(V, \in, \delta)$ and an alternating chain $\mathscr{T}$ on $\mathscr{M}$ such that both branches of $\mathscr{T}$ are $\pi$-realizable.

Proof. We are working in Morse-Kelley set theory. By Löwenheim-Skolem we have a transitive set $N$ of cardinality $\delta$ such that $V_{\delta} \cup\{\delta\} \subseteq N$ and an elementary $\tau: \mathcal{N} \rightarrow(V, \in, \delta)$ such that $\tau \uparrow\left(V_{\delta} \cup\{\delta\}\right)=$ id, where $\mathscr{N}=(N, \in, \delta)$. It will be enough to show there is a wellfounded structure $\mathscr{M}=\left(\omega, \epsilon^{\mathscr{M}}, \delta^{\mathscr{M}}\right)$ and $\pi: \mathscr{M} \rightarrow \mathscr{N}$ elementary and an alternating chain $\mathscr{T}$ on $\mathscr{M}$ and embeddings $\sigma: \mathscr{M}_{b}^{\mathscr{T}} \rightarrow \mathscr{N}$ and $\psi: \mathscr{M}_{c}^{\mathscr{T}} \rightarrow \mathscr{N}$ such that $\pi=\sigma \circ i_{0 b}^{\mathscr{T}}$ and $\pi=\psi \circ i_{0 c}^{\mathscr{I}}$. So assume toward a contradiction that there are no such $\mathscr{M}, \pi, \mathscr{T}, \sigma$, and $\psi$. Let $U$ be the natural tree (set of finite sequences closed under initial segment) of all attempts to build a tuple $(\mathscr{M}, \pi, \mathscr{T}, \sigma, \psi)$ as above. (Note that the objects to be built are to be countable. A sequence of length $n$ in $U$ should specify the first $n$ elements of $\operatorname{ran}(\pi), \operatorname{ran}(\sigma)$, and $\operatorname{ran}(\psi)$, as well as $\pi\left(E_{i}^{\mathscr{g}}\right)$ for $i<n$. This is where it helps to use wellfounded structures $\left(\omega, \in^{\mathscr{M}}, \delta^{\mathscr{M}}\right)$ rather than their transitive isomorphs.) Our assumption is that $U$ is wellfounded. Since $U$ is a tree on $N$, which has cardinality $\delta$, we can fix a set $C \subseteq V_{\delta}$ which codes $(N, U)$.

As $\delta$ is inaccessible, we can find a premouse $\mathscr{H}=\left(H, \in, \delta^{\mathscr{H}}\right)$ such that $V_{\delta^{\mathscr{L}}}^{\mathscr{H}}=V_{\delta^{\mathscr{E}}}$ and $\operatorname{cof}\left(\delta^{\mathscr{H}}\right)=\omega$ and an elementary $\varphi: \mathscr{H} \rightarrow(V, \in, \delta)$ such that $\varphi \uparrow V_{\delta^{\mathscr{E}}}$ is the identity and $C \in \operatorname{ran}(\varphi)$. Let $\varphi\left(\left(C^{*}, \mathscr{N}^{*}, U^{*}\right)\right)=$ $(C, \mathscr{N}, U)$. Now using the techniques of [MS], we can construct a normal alternating chain $\mathscr{S}$ on $(V, \in, \delta)$ such that

(i) $\delta^{\mathscr{H}}=\sup \left\{\operatorname{lh}\left(E_{i}^{\mathscr{S}}\right) \mid i \in \omega\right\}=\sup \left\{\operatorname{crit}\left(E_{i}^{\mathscr{S}}\right) \mid i \in \omega\right\}$,

(ii) $i_{0 b}^{\mathscr{S}}\left(\delta^{\mathscr{H}}\right)=\delta^{\mathscr{H}}=i_{0 c}^{\mathscr{S}}\left(\delta^{\mathscr{H}}\right)$ and $i_{0 b}^{\mathscr{S}}\left(C^{*}\right)=i_{0 c}^{\mathscr{S}}\left(C^{*}\right)$.

We use here that $\delta^{\mathscr{C}}$ is Woodin in $\mathscr{H}$, which satisfies ZFC, while $\delta^{\mathscr{H}}$ has cofinality $\omega$ in $V$. Because of (i), we cannot have $\mathscr{S} \in \mathscr{H}$; nevertheless, the 
fact that $\delta^{\mathscr{H}}$ is Woodin in $\mathscr{H}$ is enough for the construction.

Now let $\pi: \mathscr{M} \rightarrow \mathscr{N}^{*}$ be elementary, where $\mathscr{M}=\left(\omega, \epsilon^{\mathscr{M}}, \delta^{\mathscr{M}}\right)$, and choose $\pi$ and $\mathscr{M}$ so that $E_{i}^{\mathscr{S}} \in \operatorname{ran}(\pi)$ for all $i \in \omega$. Let $\mathscr{T}$ be the alternating chain on $\mathscr{M}$ whose $i$ th extender $E_{i}^{\mathscr{I}}$ is $\pi^{-1}\left(E_{i}^{\mathscr{P}}\right)$. As in the copying construction, one can easily find elementary $\sigma^{\prime}: \mathscr{M}_{b}^{\mathscr{T}} \rightarrow i_{0 b}^{\mathscr{S}}\left(\mathscr{N}^{*}\right)$ and $\psi: \mathscr{M}_{c}^{\mathscr{I}} \rightarrow i_{0 c}^{\mathscr{S}}\left(\mathscr{N}^{*}\right)$ such that $i_{0 b}^{\mathscr{S}} \circ \pi=\sigma^{\prime} \circ i_{0 b}^{\mathscr{S}}$ and $i_{0 c}^{\mathscr{S}} \circ \pi=\psi \circ i_{0 c}^{\mathscr{I}}$.

By Theorem 3.9, one of the two branches of $\mathscr{S}$ is wellfounded; assume without loss of generality that $\mathscr{P}=\left(i_{0 b}^{\mathscr{S}}(V), \in, \delta\right)$, the direct limit of $\mathscr{S}$ along $b$, is wellfounded. Now $i_{0 b}^{\mathscr{S}} \circ \pi=i_{0 b}^{\mathscr{S}}(\pi)$, since the domain of $\pi$ is not moved by $i_{0 b}^{\mathscr{S}}$. So as $\sigma^{\prime}: \mathscr{M}_{b}^{\mathscr{T}} \rightarrow i_{0 b}^{\mathscr{S}}\left(\mathscr{N}^{*}\right)$ and $i_{0 b}^{\mathscr{S}}(\pi)=\sigma^{\prime} \circ i_{0 b}^{\mathscr{S}}$, the tree in $\mathscr{P}$ which searches for an object like $\sigma^{\prime}$ has a branch in $\mathscr{P}$, so $\mathscr{P} \vDash \exists \sigma^{\prime}\left(\sigma^{\prime}\right.$ : $\left.\mathscr{M}_{b}^{\mathscr{I}} \rightarrow i_{0 b}^{\mathscr{S}}\left(\mathscr{N}^{*}\right) \wedge i_{0 b}^{\mathscr{S}}(\pi)=\sigma^{\prime} \circ i_{0 b}^{\mathscr{I}}\right)$. The elementarity of $i_{0 b}^{\mathscr{S}}$ then gives us an elementary $\sigma: \mathscr{M}_{b}^{\mathscr{T}} \rightarrow \mathscr{N}^{*}$ such that $\pi=\sigma \circ i_{0 b}^{\mathscr{T}}$ :

Now $\mathscr{M}, \pi, \mathscr{T}$, and $\sigma$ satisfy all the requirements on the first four coordinates of a branch of $U^{*}$. Now $\psi: \mathscr{M}_{c}^{\mathscr{T}} \rightarrow i_{0 c}^{\mathscr{S}}\left(\mathscr{N}^{*}\right)$ and $i_{0 c}^{\mathscr{S}}(\pi)=$ $i_{0 c}^{\mathscr{S}} \circ \pi=\psi \circ i_{0 c}^{\mathscr{T}}$. It follows that $\left(\mathscr{M}, i_{0 c}^{\mathscr{S}}(\pi), \mathscr{T}, i_{0 c}^{\mathscr{S}}(\sigma), \psi\right)$ determines a branch through $i_{0 c}^{\mathscr{S}}\left(U^{*}\right)$, so that $i_{0 c}^{\mathscr{S}}\left(U^{*}\right)$ is illfounded (in $V$, not necessarily in $\left.i_{0 c}^{\mathscr{S}}(V)\right)$. On the other hand, $U^{*}$ is wellfounded, and so $i_{0 b}^{\mathscr{S}}\left(U^{*}\right)$ is wellfounded in $\mathscr{P}$ and, hence, wellfounded in $V$. As $i_{0 b}^{\mathscr{S}}\left(C^{*}\right)=i_{0 c}^{\mathscr{S}}\left(C^{*}\right)$, we have $i_{0 b}^{\mathscr{S}}\left(U^{*}\right)=i_{0 c}^{\mathscr{S}}\left(U^{*}\right)$. This contradiction completes the proof.

One might ask what happens if we require that our base model be more like $V$-for example, by requiring that it contain all the ordinals. The following argument, due to Woodin, shows this still does not guarantee unique wellfounded branches.

Proposition 5.2 (Woodin). Let $\delta$ be Woodin, and assume $V_{\delta}^{\#}$ exists. Then there is an ordinal $\gamma<\delta$ and an alternating chain $\mathscr{T}$ on $L\left(V_{\gamma}\right)$ such that both branches of $\mathscr{T}$ are wellfounded.

Proof. Again, for simplicity, we work in Morse-Kelley. By Löwenheim-Skolem we can find a premouse $\mathscr{H}$ and an elementary $\varphi: \mathscr{H} \rightarrow(V, \in, \delta)$ such that, setting $\gamma=\delta^{\mathscr{H}}, V_{\gamma}^{\mathscr{H}}=V_{\gamma}$ and $\operatorname{cof}(\gamma)=\omega$. Since $\delta \in \operatorname{ran}(\varphi), V_{\delta}^{\#} \in \operatorname{ran}(\varphi)$; and $\varphi^{-1}\left(V_{\delta}^{\#}\right)=V_{\gamma}^{\#}$ because $\varphi^{-1}\left(V_{\delta}^{\#}\right)$ is embedded by $\varphi$ into an "iterable" character. Now the methods of [MS] yield an alternating chain $\mathscr{S}$ on $V$ such that $\gamma=\sup \left\{\operatorname{lh}\left(E_{i}^{\mathscr{S}}\right) \mid i \in \omega\right\}=\sup \left\{\operatorname{crit}\left(E_{i}^{\mathscr{S}}\right) \mid i \in \omega\right\}, i_{0 b}^{\mathscr{S}}(\gamma)=i_{0 c}^{\mathscr{S}}(\gamma)=\gamma$, and $i_{0 b}^{\mathscr{S}}\left(V_{\gamma}^{\#}\right)=i_{0 c}^{\mathscr{S}}\left(V_{\gamma}^{\#}\right)$.

Let $\mathscr{T}$ be the same as $\mathscr{S}$ but have base model $L\left(V_{\gamma}\right)$; that is, $E_{i}^{\mathscr{T}}=$ $E_{i}^{\mathscr{S}}$ for $i \in \omega$, but $\mathscr{M}_{0}^{\mathscr{T}}=L\left(V_{\gamma}\right)$ whereas $\mathscr{M}_{0}^{\mathscr{S}}=V$. (This is an abuse of notation, but context will not determine the base model in this situation.) Assume toward a contraction that, for example, $\mathscr{M}_{b}^{\mathscr{T}}$ is illfounded. Let $\nu$ be the $\omega_{1 \mathrm{st}}$ indiscernible of $L\left(V_{\gamma}\right)$. By Löwenheim-Skolem and indiscernibility, 
$i_{0 b}^{\mathscr{T}}(\nu)$ is illfounded. Since there is a natural elementary $\sigma: \mathscr{M}_{b}^{\mathscr{G}} \rightarrow L\left(V_{\gamma}\right)^{\mathscr{K}_{b}^{\mathscr{S}}}$ such that $\sigma \circ i_{0 b}^{\mathscr{S}}=i_{0 b}^{\mathscr{S}} \uparrow L\left(V_{\gamma}\right)$, we have that $i_{0 b}^{\mathscr{S}}(\nu)$ is illfounded. On the other hand, let $W$ be a prewellorder of $V_{\gamma}$ of order type $\nu$ coded naturally in $V_{\gamma}^{\#}$. Since $i_{0 b}^{\mathscr{S}}\left(V_{\gamma}^{\#}\right)=i_{0 c}^{\mathscr{S}}\left(V_{\gamma}^{\#}\right), i_{0 b}^{\mathscr{S}}(W)=i_{0 c}^{\mathscr{S}}(W)$. Since $\mathscr{M}_{c}^{\mathscr{S}}$ is wellfounded, $i_{0 c}^{\mathscr{S}}(W)$ is wellfounded. As $i_{0 b}^{\mathscr{S}}(W)$ has order type $i_{0 b}^{\mathscr{S}}(\nu)$, which is illfounded, we have a contradiction.

Although we cannot prove $\mathrm{UBH}$, we can show $\neg \mathrm{UBH}$ has some consistency strength. Theorem 2.2 shows that $\neg \mathrm{UBH}$ implies that there is an ordinal $\delta$ such that $\delta$ is Woodin in $L\left(V_{\delta}\right)$. We shall now push a little further and show that there is a $\delta$ such that $V_{\delta}^{\#}$ exists and $\delta$ is Woodin in $L\left(V_{\delta} \cup\left\{V_{\delta}^{\#}\right\}\right)$.

Lemma 5.3. Let $\mathscr{T}$ be an iteration tree of limit length $\lambda$ on a proper class premouse $\mathscr{M}$, and suppose $\mathscr{T}$ has distinct cofinal wellfounded branches $b$ and c. Let $\theta=\sup \left\{\rho^{\mathscr{T}}(\alpha, \lambda) \mid \alpha<\lambda\right\}$; then $\left(V_{\theta}^{\mathscr{M}_{b}}\right)^{\#}$ exists.

Proof. We adopt the notation from the proof of Theorem 2.2. Let $\Gamma$ be the class of all strong limit cardinals of cofinality $>\max (\theta, \lambda)$. If $\alpha \in \Gamma$, then $\alpha$ is an indiscernible of $L\left(V_{\mu}^{\mathscr{M}_{b}}\right)$ for all $\mu<\theta$ (notice that $\mu<\theta \Rightarrow\left(V_{\mu}^{\mathscr{M}_{b}}\right)^{\#}$ exists). Moreover, if $\alpha \in \Gamma$ then $i_{0 b}(\alpha)=i_{0 c}(\alpha)=\alpha$. Let $\mu=\mu_{n}=\inf \left\{\kappa_{n}, \nu_{n}\right\}$ be as in (a) of the claim in the proof of Theorem 2.2. We assume $n$ is large enough that $\theta \in \operatorname{ran}\left(i_{\delta_{n}, b}\right) \cap \operatorname{ran}\left(i_{\gamma_{n}, c}\right)$. Let $x \in H$ iff $x \in L\left(V_{\theta}^{\mathscr{M}_{b}}\right)$ and $x$ is definable over $L\left(V_{\theta}^{\mathscr{M}_{b}}\right)$ from parameters in $\left(V_{\mu}^{\mathscr{M}_{b}}\right) \cup \Gamma \cup\{\theta\}$. Then (a) of the claim implies $H \prec L\left(V_{\theta}^{\mathscr{M}_{b}}\right)$ and $H \cap V_{\theta}^{\mathscr{M}_{b}}=V_{\mu}^{\mathscr{K}_{b}}$. Thus $H$ collapses to $L\left(V_{\mu}^{\mathscr{M}_{b}}\right)$; let $\pi: L\left(V_{\mu}^{\mathscr{M}_{b}}\right) \rightarrow L\left(V_{\theta}^{\mathscr{M}_{b}}\right)$ be the collapse map. Letting $\Delta=\{\alpha \mid \alpha$ is the $\alpha$ th ordinal in $\Gamma\}$, we have $\pi \uparrow \Delta=\mathrm{id}$, and since the ordinals in $\Delta$ are indiscernible over $L\left(V_{\mu}^{\mathscr{M}_{b}}\right)$ with respect to parameters from $V_{\mu}^{\mathscr{M}_{b}}$, they are indiscernible over $L\left(V_{\theta}^{\mathscr{M}_{b}}\right)$ with respect to parameters from $V_{\mu}^{\mathscr{M}_{b}}$. But this is true for every value $\mu_{n}$ of $\mu$, and $\sup \left\{\mu_{n} \mid n \in \omega\right\}=\theta$. Thus the ordinals in $\Delta$ are indiscernible over $L\left(V_{\theta}^{\mathscr{M}_{b}}\right)$ with respect to arbitrary parameters in $V_{\theta}^{\mathscr{M}_{b}}$, and $\left(V_{\theta}^{\mathscr{M}_{b}}\right)^{\#}$ exists.

Theorem 5.4. If $\mathrm{UBH}$ is false, then there is a $\delta$ such that $V_{\delta}^{\#}$ exists and $\delta$ is Woodin in $L\left(V_{\delta} \cup\left\{V_{\delta}^{\#}\right\}\right)$.

Proof. Let $\mathscr{T}, \theta$, etc., be as in Lemma 5.3, with $\mathscr{M}_{0}=V$. It is enough to show that $\left(V_{\theta}^{\mathscr{M}_{b}}\right)^{\#} \in \mathscr{M}_{b} \cap \mathscr{M}_{c}$, and for this it is enough to show that both $\mathscr{M}_{b}$ and $\mathscr{M}_{c}$ satisfy “ $V_{\theta}^{\#}$ exists".

Suppose, for example, that $\mathscr{M}_{b} \vDash$ " $V_{\theta}^{\#}$ does not exist". Then as $i_{0 b}$ is elementary, $V_{\gamma}^{\#}$ does not exist for some $\gamma$. Let $\delta$ be the least such $\gamma$, so that $i_{0 b}(\delta) \leq \theta$. Now for all $\mu<\theta, \mathscr{M}_{b} \models$ “ $V_{\mu}^{\#}$ exists", so $i_{0 b}(\delta)=\theta$. But then 
$i_{0 b}$ is elementary from $L\left(V_{\delta}\right)$ to $L\left(V_{\theta}^{\mathscr{M}_{b}}\right)$ and has a stationary class of fixed points, so $V_{\delta}^{\#}$ exists. This is a contradiction.

Thus $\left(V_{\theta}^{\mathscr{M}_{b}}\right)^{\#} \in \mathscr{M}_{b} \cap \mathscr{M}_{c}$. By Theorem 2.2, $L\left(V_{\theta}^{\mathscr{M}_{b}} \cup\left\{\left(V_{\theta}^{\mathscr{M}_{b}}\right)^{\#}\right\}\right)$ satisfies “ $\theta$ is Woodin". This is a fact about $\mathscr{M}_{b}$; pulling it back to $V$ via $i_{0 b}$, we are done.

With a little more work, one can replace $V_{\delta}^{\#}$ by $V_{\delta}^{\dagger}$ in Theorem 5.4. To go much further along this line, one seems to need core model theory. The second author has recently extended the Dodd-Jensen-Mitchell theory to models having a Woodin cardinal and applied the extended theory in showing that if there is a measurable cardinal $\kappa$ and a tree $\mathscr{T}$ on $V_{\kappa}$ witnessing the failure of UBH, then there is an inner model with two Woodin cardinals. Almost certainly, the measurable cardinal is required by the mathematician and not by the mathematics. On the other hand, there seems to be a real obstacle to using core model theory to get more than an inner model with two Woodin cardinals from $\neg$ UBH. So it seems mildly plausible that $\neg \mathrm{UBH}$, if consistent at all, is equiconsistent with the existence of two Woodin cardinals.

$\mathrm{H}$. Woodin independently rediscovered part of the proof of Lemma 5.3 and noticed that it yields the following.

Theorem 5.5 (Woodin). Suppose that $\forall \gamma<\delta\left(L\left(V_{\gamma}\right) \models\right.$ “ $\gamma$ is not Woodin"); then every iteration tree on $L\left(V_{\delta}\right)$ has at most one cofinal wellfounded branch.

Proof. Suppose $\mathscr{T}$ on $L\left(V_{\delta}\right)$ has distinct cofinal wellfounded branches $b$ and $c$, and let $\theta=\sup \{\rho(\alpha, \ln (\mathscr{T})) \mid \alpha<\operatorname{lh}(\mathscr{T})\}$. So $\theta$ is Woodin in $L\left(V_{\theta}^{\mathscr{M}_{b}}\right)$. The proof of Lemma 5.3 shows there is a $\mu<\theta$ and elementary $\pi: L\left(V_{\mu}^{\mathscr{M}_{b}}\right) \rightarrow$ $L\left(V_{\theta}^{\mathscr{M}_{b}}\right)$ such that $\pi(\mu)=\theta$. So $\mu$ is Woodin in $L\left(V_{\mu}^{\mathscr{M}_{b}}\right)$. Since $\mathscr{M}_{0}=L\left(V_{\delta}\right)$ satisfies "at most one $\gamma$ is Woodin in $L\left(V_{\gamma}\right)$ " and $i_{0 b}$ is elementary, we have a contradiction.

B. Cofinal branches. The Cofinal Branches Hypothesis, or $\mathrm{CBH}$, is the following.

CBH. Let $\mathscr{T}$ be an iteration tree on $V$. Then

(a) if $\mathscr{T}$ has limit length, then $\mathscr{T}$ has a cofinal wellfounded branch; and

(b) if $\mathscr{M}_{\alpha}^{\mathscr{T}} \models$ “ $E$ is an extender" and if $\beta<\alpha$ and $\operatorname{crit}(E)<\rho^{\mathscr{T}}(\beta, \alpha)$, then Ult $\left(\mathscr{M}_{\beta}^{\mathscr{T}}, E\right)$. is wellfounded.

As with the UBH, it seems important that the base model be $V$. We have an analog to Proposition 5.1: If there are two Woodin cardinals and a measurable cardinal above them both, then there is a countable $\mathscr{M} \preceq V$ and countable iteration tree $\mathscr{T}$ on $\mathscr{M}$ such that (a) or (b) of CBH fails. (Otherwise, there would be an inner model of this large cardinal hypothesis with a $\Delta_{4}^{1}$ wellorder of its reals, contrary to [ShW] and [MS]. We shall show this in a sequel to this paper. One cannot have too direct a construction of such an $\mathscr{M}$ and $\mathscr{T}$ because Theorem 4.3 implies $\ln (\mathscr{T})>\omega$.) A similar argument gives under the same large cardinal hypothesis an analog of Proposition 5.2: There is a proper 
class premouse $\mathscr{M}$ and countable iteration tree $\mathscr{T}$ on $\mathscr{M}$ such that (a) or (b) of CBH fails. (The claims in this paragraph have not been checked in detail.)

$\mathrm{CBH}$ is open even for iteration trees of length $\omega$. Let us restrict ourselves to plus one trees, the case of interest in applications. By Theorem 3.9, it would suffice to show that if $\mathscr{T}$ is a plus one tree on $V$ of length $\omega$ and $\mathscr{T}$ has no cofinal wellfounded branches, then $\mathscr{T}$ is continuously illfounded. In this direction we know the following. Let us say a set $M$ is $\kappa$-closed iff ${ }^{\kappa} M \subseteq M$, and let us say an extender $E$ is $\kappa$-closed iff $\operatorname{Ult}(V, E)$ is $\kappa$-closed.

Theorem 5.6. Let $\mathscr{M}$ be $2^{\aleph_{0}}$ closed, and let $\mathscr{T}$ be an iteration tree of countable limit length $\lambda$ on $\mathscr{M}$ such that $\forall \alpha<\lambda\left(\mathscr{M}_{\alpha}^{\mathscr{T}} \models\right.$ “ $E_{\alpha}^{\mathscr{T}}$ is $2^{\aleph_{0}}$-closed"). If $\mathscr{T}$ has no cofinal wellfounded branch, then $\mathscr{T}$ is continuously illfounded.

Proof. We consider first the case $\lambda=\omega$. Let $B=\{a \subseteq \omega \mid a$ is linearly ordered by $\mathrm{T}$, and no proper superset of $a$ is linearly ordered by $\mathrm{T}\}$. For each $a \in B$, pick ordinals $\theta_{i}^{a}$, for $i \in a$, so that for $m, n \in a$ with $m \mathrm{~T} n, i_{m n}^{\mathscr{I}}\left(\theta_{m}^{a}\right)>\theta_{n}^{a}$. By $2^{\aleph_{0}}$-closure, $B$ and the map $(i, a) \mapsto \theta_{i}^{a}$ are members of $\mathscr{M}_{0}^{\mathscr{T}}$.

Let $\theta=\sup \left\{\theta_{0}^{a} \mid a \in B\right\}$ so that $\theta \in \mathscr{M}_{0}^{\mathscr{T}}$. Let $X$ be the set of pairs $(i, f)$ such that $i \in \omega$ and $f:\{a \in B \mid i \in a\} \rightarrow \theta$. We define a binary relation $R$ on $X$ by

$$
(i, f) R(j, g) \Longleftrightarrow[j \mathrm{~T} i \text { and } \forall a \in \operatorname{dom}(f)(f(a)<g(a))] .
$$

It is easy to see that $R$ is wellfounded. Notice $X$ and $R$ are in $\mathscr{M}_{0}^{\mathscr{T}}$.

A simple induction shows that $\mathscr{M}_{k}^{\mathscr{T}}$ is $2^{\aleph_{0}}$-closed for all $k<\omega$. So if we set $f_{k}(a)=\theta_{k}^{a}$ for all $a \in B$ such that $k \in a$, then $f_{k} \in \mathscr{M}_{k}^{\mathscr{T}}$, and in fact $\left(k, f_{k}\right) \in i_{0, k}^{\mathscr{T}}(X)$. Let $\theta_{k}$ be the rank of $\left(k, f_{k}\right)$ in $i_{0 k}^{\mathscr{I}}(R)$. It is easy to see that if $k \mathrm{~T} j$, then $\left(j, f_{j}\right) i_{k j}^{\mathscr{T}}(R)\left(k, i_{k j}^{\mathscr{T}}\left(f_{k}\right)\right)$, so that $i_{k j}^{\mathscr{T}}\left(\theta_{k}\right)>\theta_{j}$. Thus the $\theta_{k}$ 's witness the continuous illfoundedness of $\mathscr{T}$.

The only real difficulty in generalizing this proof to the case $\lambda>\omega$ lies in the fact that $\mathscr{M}_{\alpha}^{\mathscr{T}}$ will not be $2^{\aleph_{0}}$ closed when $\alpha \geq \omega$. However, there is a natural notion of finite support for an element of $\mathscr{M}_{\alpha}^{\mathscr{T}}$, and this enables us to write $\mathscr{M}_{\alpha}^{\mathscr{T}}=\bigcup_{i \in \omega} \mathscr{M}_{\alpha, i}^{\mathscr{T}}$, where each $\mathscr{M}_{\alpha, i}^{\mathscr{T}}$ is a $2^{\aleph_{0}}$-closed elementary submodel of $\mathscr{M}_{\alpha}^{\mathscr{T}}$. We can then choose $\theta_{\alpha}^{a}$ 's so that there is a fixed $i \in \omega$ such that $\theta_{\alpha}^{a} \in \mathscr{M}_{\alpha, i}^{\mathscr{T}}$ for all $a \in B$ with $\alpha \in a$. With these changes, the $\lambda=\omega$ proof goes through.

Corollary 5.7. Let $\mathscr{T}$ be a plus one iteration tree of length $\omega$ on $V$ such that $\forall \alpha<\omega\left(\mathscr{M}_{\alpha}^{\mathscr{T}} \models “ E_{\alpha}^{\mathscr{T}}\right.$ is $2^{\aleph_{0}}$-closed"). Then $\mathscr{T}$ has a cofinal wellfounded branch.

One can characterize continuous illfoundedness as the nonexistence of "generic" cofinal wellfounded branches.

Theorem 5.8. Let $\mathscr{T}$ be an iteration tree of countable limit length on $\left(V_{\alpha}\right.$, $\epsilon, \delta)$, and suppose there is a measurable cardinal $>\alpha$. The following are equivalent: 
(1) $\mathscr{T}$ is continuously illfounded,

(2) if $G$ is generic for the Levy collapse making $V_{\alpha}$ countable, then $V[G] \vDash$ “ $\mathscr{T}$ has no cofinal wellfounded branch".

Proof. The nontrivial direction is $(2) \rightarrow(1)$. Let $\kappa>\alpha$ be measurable. Let $H$ be generic for the stationary tower forcing up to $\kappa$, with $P_{\omega_{1}}\left(V_{\alpha+1}\right) \in H$ (cf. $[W]$ ). So we have an elementary $j: V_{\kappa} \rightarrow V_{\kappa}[H]$, and $V_{\alpha+1}$ is countable in $V_{\kappa}[H]$. In $V_{\kappa}[H]$ we have $G$ generic over $V$ for the Levy collapse making $V_{\alpha}$ countable, and as $L_{\kappa}\left[V_{\alpha}, G\right]$ is $\Sigma_{2}^{1}$ absolute in $V_{\kappa}[H], \mathscr{T}$ has no cofinal wellfounded branches in $V_{\kappa}[H]$. But now $\mathscr{M}=\left(V_{\alpha}, \in, \delta\right)$ is countable in $V_{\kappa}[H]$, and $\mathscr{M} \leq\left(j\left(V_{\alpha}\right), \in, j(\delta)\right)$, via $j \uparrow V_{\alpha}^{\alpha}$, and $j \uparrow V_{\alpha} \in V_{\kappa}[H]$. So in $V_{\kappa}[H]$ we can use the proof that Lemma 4.6 implies Theorem 4.3 (see Claim 1 of that proof) to conclude that $j(\mathscr{T})=j \mathscr{T}$ is continuously illfounded on $\left(j\left(V_{\alpha}\right), \in, j(\delta)\right)$. As $j$ is elementary, $\mathscr{T}$ is continuously illfounded on $\left(V_{\alpha}, \in, \delta\right)$.

Woodin has found a more elementary proof of Theorem 5.8 which uses the existence of $V_{\alpha}^{\#}$ instead of the measurable cardinal $>\alpha$.

As with the $\mathrm{UBH}$, we can show that $\mathrm{CBH}$ is true for iteration trees on $L\left(V_{\delta}\right)$, where $\delta$ is small enough that no $\gamma<\delta$ is Woodin in $L\left(V_{\gamma}\right)$. We need a large cardinal hypothesis for this result. The theorem was proved independently by Woodin under a weaker large cardinal hypothesis.

Theorem 5.9. Suppose $\forall \gamma<\delta\left(L\left(V_{\gamma}\right) \models\right.$ " $\gamma$ is not Woodin"), and $\mathscr{T}$ is an iteration tree on $L\left(V_{\delta}\right)$. Suppose $\mathscr{T} \in V_{\kappa}$ for some Woodin cardinal $\kappa>\delta$ such that $V_{\kappa}^{\#}$ exists. Then (a) and (b) of the conclusion of $\mathrm{CBH}$ hold at $\mathscr{T}$.

Proof. Suppose $\mathscr{T}$ is a counterexample. Let $\mathscr{T} \in V_{\mu}$, where $\mu$ is measurable, and $\delta<\mu<\kappa$. Let $\mu<\alpha<\kappa$ where $\left(V_{\alpha}, \in, \delta\right)=\mathscr{P}$ is a premouse. Let $G$ be generic for the full stationary tower forcing up to $\kappa$, with $P_{\omega_{1}}\left(V_{\alpha}\right) \in G$, and let $j: V \rightarrow M$ be the generic elementary embedding. Thus $j(\kappa)=\kappa$, and ${ }^{\lambda} M \subseteq M$ in $V[G]$ for all $\lambda<\kappa$. Now $\mathscr{T}$ and $\mathscr{P}$ are countable in $V[G]$, and $j \uparrow V_{\alpha}$ embeds $\mathscr{P}$ elementarily into $\left(j\left(V_{\alpha}\right), \in, j(\delta)\right)$, and $j\left(V_{\alpha}\right)=V_{j(\alpha)}^{V[G]}$, so we can apply Theorem 4.3 in $V[G]$ to get a maximal branch $b$ of $\mathscr{T}$ and an elementary $\sigma: \mathscr{P}_{b}^{\mathscr{T}} \rightarrow\left(j\left(V_{\alpha}\right), \in, \delta\right)$. (Here we are thinking of $\mathscr{T}$ as a tree on $\mathscr{P}$.)

We claim $b$ is a wellfounded branch of $\mathscr{T}$ when $\mathscr{T}$ is regarded as a tree on $L\left(V_{\delta}\right)$. For let $\mathscr{U}$ be a normal ultrafilter on $\mu$. The existence of $\sigma$ means $\mathscr{P}_{b}^{\mathscr{T}}$ can be iterated OR times by $i_{0 b}^{\mathscr{T}}(\mathscr{U})$; let $\mathscr{N}$ be this iterate. A simple calculation, which we shall omit, shows that $\mathscr{N}$ is isomorphic to the result of iterating $\mathscr{P}$ OR times via $\mathscr{U}$, then regarding $\mathscr{T}$ as a tree on the direct limit $Q$, and forming $Q_{b}^{\mathscr{T}}$. (Notice here $\delta<\mu$.) So $Q_{b}^{\mathscr{T}}=\mathscr{N}$ is wellfounded. But $L\left(V_{\delta}\right) \subseteq Q$, so $b$ is wellfounded when $\mathscr{T}$ is regarded as a tree on $L\left(V_{\delta}\right)$.

Let us fix a real $x \in V[G]$ which is generic over $V$ for the Levy collapse making some $V_{\beta}$ with $\delta, \mathscr{T} \in V_{\beta}$ countable. Assume $x \operatorname{codes}\left(V_{\delta}, \mathscr{T}\right)$ in some natural way. It is a $\Sigma_{3}^{1}$ assertion about $x$, true in $V[G]$, that $\mathscr{T}$ has a 
maximal wellfounded branch when regarded as a tree on $L\left(V_{\delta}\right)$. By MartinSolovay absoluteness (cf. [MSo]), this assertion is true in $V[x]$. The proof of Theorem 5.5, applied in $V[x]$, shows that $\mathscr{T}$ has at most one maximal branch in $V[x]$ when regarded as a tree on $L\left(V_{\delta}\right)$. As the collapse poset is homogeneous, $\mathscr{T}$ has a maximal wellfounded branch $b$ in $V$. By Theorem $5.5, b$ is cofinal, and thus $\mathscr{T}$ is not a counterexample to the theorem. Contradiction.

Woodin proved Theorem 5.9 under the hypothesis that $\left(\mathscr{T}, V_{\delta}\right)^{\#}$ exists.

C. Iteration strategies. In practice, iteration trees $\mathscr{T}$ on $\mathscr{M}$ are built inductively as follows. At successor steps $\alpha+1, E_{\alpha}^{\mathscr{T}}$ and T-pred $(\alpha+1)$ are determined by the problem at hand, in a way over which we have no control. At limit steps $\lambda$, it is "up to us" to choose a cofinal wellfounded branch of $\mathscr{T} \uparrow \lambda$. (This is how the comparison process of inner model theory goes; see Lemma 6.7.) A method for making the choices which are ours can be regarded as a strategy in a certain game.

Let $\mathscr{M}$ be a premouse and $\theta$ an ordinal, $\theta>1$. The iteration game of length $\theta$ on $\mathscr{M}$ is played as follows. There are two players, I and II. For $1 \leq \beta<\theta$, player I makes move $\beta$ if $\beta$ is a successor ordinal, and player II makes move $\beta$ if $\beta$ is a limit ordinal. The moves preceding the $\beta$ th determine an iteration tree $\mathscr{T}_{\beta}$ of length $\beta$ on $\mathscr{M}$, and $\gamma<\eta \Rightarrow \mathscr{T}_{\gamma}=\mathscr{T}_{\eta} \uparrow \gamma . \mathscr{T}_{0}$ is the unique length one tree on $\mathscr{M} ; \mathscr{M}_{0}^{\mathscr{T}_{0}}=\mathscr{M}$, and $E_{0}^{\mathscr{T}_{0}}$ is undefined. Now suppose $\beta=\alpha+1$. Player I's $\beta$ th move must consist of an extender $E \in \mathscr{M}_{\alpha}^{\mathscr{T}_{\beta}}$ with $\operatorname{crit}(E)<\delta^{\mathscr{M}_{a}^{\mathcal{I}_{\beta}}}$ and an ordinal $\gamma \leq \alpha$ such that $\left(\gamma<\alpha \Rightarrow \operatorname{crit}(E)<\rho^{\stackrel{\alpha}{\mathscr{I}_{\beta}}}(\gamma, \alpha)\right)$. If he cannot make such a move, I loses. Let $\mathscr{P}=\operatorname{Ult}(Q, E)$, where $Q=\mathscr{M}_{\gamma}^{\mathscr{I}_{\beta}}$. If $\mathscr{P}$ is illfounded, then II loses. Otherwise, let $\mathscr{T}_{\beta+1}$ be the unique length $\beta+1$ tree $\mathscr{T}$ extending $\mathscr{T}_{\beta}$ such that $E_{\beta}^{\mathscr{T}}=E$ and $\mathrm{T}-\operatorname{pred}(\beta)=\gamma$; this completes round $\beta$. Next, suppose $\beta$ is a limit ordinal. Let $\mathscr{T}$ be the unique length $\beta$ iteration tree such that $\gamma<\beta \Rightarrow \mathscr{T}_{\gamma}=\mathscr{T} \uparrow \gamma$. Player II's $\beta$ th move must be a cofinal branch $b$ of $\mathscr{T}$ such that $\mathscr{M}_{b}^{\mathscr{T}}$ is wellfounded; if he fails to make such a move, II loses. If he succeeds, let $\mathscr{T}_{\beta+1}$ be the unique length $\beta+1$ tree $\mathscr{S}$ extending $\mathscr{T}$ such that $[0, \beta)_{S}=b$.

If II does not lose the iteration game at some move $\beta<\theta$, then he wins it.

Definition 5.10. $\mathscr{G}(\mathscr{M}, \theta)$ is the iteration game of length $\theta$ on $\mathscr{M}$ defined above. $\mathscr{M}$ is $\theta$-iterable iff II has a winning strategy in $\mathscr{G}(\mathscr{M}, \theta) . \mathscr{M}$ is fully iterable iff $\mathscr{M}$ is $\theta$-iterable for all $\theta$.

We call a winning strategy for II in $\mathscr{G}(\mathscr{M}, \theta)$ a $\theta$-iteration strategy for $\mathscr{M}$.

There is an obvious modification of $\mathscr{G}(\mathscr{M}, \theta)$ in which the players can only produce normal iteration trees on $\mathscr{M}$ : at move $\alpha+1$ player I must play $\rho_{\alpha}$ such that $\rho_{\gamma}<\rho_{\alpha}$ for $\gamma<\alpha$ and $\rho_{\alpha}+2 \leq \operatorname{strength}(E)$ and must set T-pred $(\alpha+1)=$ least $\eta$ such that $\operatorname{crit}(E) \leq \rho_{\eta}$. (In this game, I's chances of losing because he has nothing to play are significantly greater.) Let $\mathscr{G}^{*}(\mathscr{M}, \theta)$ be this restricted game, and say $\mathscr{M}$ is normally $\theta$-iterable iff II has a winning strategy in $\mathscr{G}^{*}(\mathscr{M}, \theta)$, etc. 
CBH is just the assertion that for all $\theta$, II wins $\mathscr{G}(V, \theta)$ by simple minded quasi strategy of choosing any cofinal wellfounded branch of the tree built so far. (UBH asserts that there is no more clever iteration strategy.) Note, however, that in contrast to the situation with $\mathrm{CBH}, \theta$-iterability is preserved by going to an elementary submodel. For if $\pi: \mathscr{N} \rightarrow \mathscr{M}$ is elementary and II wins $\mathscr{G}(\mathscr{M}, \theta)$ via $\Sigma$, then II can win $\mathscr{G}(\mathscr{N}, \theta)$ by making sure that if $\mathscr{T}$ is the iteration tree on $\mathscr{N}$ being played, then $\pi \mathscr{T}$ is a play according to $\Sigma$. The Strategic Branches Hypothesis, or SBH, is the following.

SBH. $V$ is fully iterable.

The only partial result we have on SBH which goes beyond those on $\mathrm{CBH}$ is the following: Let $\mathscr{M} \preceq\left(V_{\alpha}, \in, \delta\right)$ for some $\alpha, \delta$, and let $\mathscr{M}$ be countable. Suppose there is a measurable cardinal. Then $\mathscr{M}$ is normally $\left(\omega^{2}+1\right)$-iterable. (One shows directly that I has no winning strategy in $\mathscr{G}^{*}\left(\mathscr{M}, \omega^{2}+1\right)$ and then uses the measurable cardinal to conclude II has a winning strategy. We omit further detail.)

The following immediate corollary of Corollary 2.3 and Theorem 4.3 will play a role in $\S 6$.

Corollary 5.11. Let $\mathscr{M} \preceq\left(V_{\alpha}, \in, \delta\right)$ be countable, where $\left(V_{\alpha}, \in, \delta\right)$ is a premouse such that $\forall \gamma<\delta\left(L_{\alpha}\left(V_{\gamma}\right) \not \neq\right.$ “ $\gamma$ is Woodin"). Then $\mathscr{M}$ is normally $\omega_{1}$-iterable.

Proof. We claim that the following is a winning strategy for player II in $\mathscr{G}^{*}\left(\mathscr{M}, \omega_{1}\right):$ if $\lambda<\omega_{1}$ is a limit ordinal and the play of the game so far has produced $\mathscr{T}$ on $\mathscr{M}$ of length $\lambda$, pick a cofinal wellfounded branch $b$ of $\mathscr{T}$ such that $\mathrm{OR}^{\mathscr{M}_{b}^{\mathcal{G}}}$ is as small as possible and play it. Suppose this strategy loses at stage $\beta<\omega_{1}$ against some play by $\mathrm{I}$, and let $\mathscr{T}$ be the iteration tree on $\mathscr{M}$ produced during the stages before the $\beta$ th. From Theorem 4.3 we get that $\mathscr{T}$ has a maximal wellfounded branch $b$ such that $\sup b=\lambda\langle\beta$. Let $\mathscr{S}=\mathscr{T} \uparrow \lambda$ and $c=\{\alpha \mid \alpha \mathrm{T} \lambda\}$; then $b$ and $c$ are distinct cofinal wellfounded branches of $\mathscr{S}$. Notice that II's strategy has ensured that $\mathrm{OR} \cap \mathscr{M}_{c}^{\mathscr{T}} \leq \mathrm{OR} \cap \mathscr{M}_{b}^{\mathscr{T}}$. Letting $\theta=\sup \left\{\rho^{\mathscr{T}}(\eta, \lambda) \mid \eta<\lambda\right\}$, we have from Corollary 2.3 that $\mathscr{M}_{c}^{\mathscr{T}} \vDash$ " $\theta$ is Woodin in $L\left(V_{\theta}\right)$ ", so $\theta=\delta^{\mathscr{M}_{c}^{\mathcal{I}}}$ by our minimality hypothesis on $\left(V_{\alpha}, \in, \delta\right)$.

Let $\rho_{\alpha}$, for $\alpha<\operatorname{lh}(\mathscr{T})$, witness the normality of $\mathscr{T}$. One can easily check that $\theta=\sup \left\{\rho_{\alpha} \mid \alpha<\lambda\right\}$. (Recall here that $\theta$ is also the sup of the critical points along either $b$ or $c$, so that $\theta \leq \sup \left\{\rho_{\alpha} \mid \alpha<\lambda\right\}$.) The rules of $\mathscr{G}^{*}\left(\mathscr{M}, \omega_{1}\right)$ required I to play $E_{\lambda}^{\mathscr{T}} \in V_{\theta}^{\mathscr{M}_{c}^{\mathscr{T}}}$ and $\rho_{\lambda}<\operatorname{lh}\left(E_{\lambda}^{\mathscr{T}}\right)$ such that $\theta \leq \rho_{\lambda}$. This is clearly impossible, a contradiction.

Inspecting the proof of Corollary 5.11, we see that what we get from Theorem 4.3 , in the case $\mathscr{M} \preceq\left(V_{\alpha}, \in, \delta\right)$ is countable, is a winning strategy for II in a game less demanding of him than $\mathscr{G}^{*}\left(\mathscr{M}, \omega_{1}\right)$. We call this less-demanding game the weak iteration game (of length $\left.\omega_{1}\right)$ on $\mathscr{M}$ or $\mathscr{W} G^{*}\left(\mathscr{M}, \omega_{1}\right)$.

Let us call a system $\mathscr{T}$ on a premouse $\mathscr{P}$ a putative normal iteration tree on $\mathscr{P}$ just in case $\mathscr{T}$ has all the properties of a normal iteration tree on $\mathscr{P}$, 
except that we allow the last model of $\mathscr{T}$, if it has one, to be illfounded. The game $\mathscr{W} G^{*}\left(\mathscr{M}, \omega_{1}\right)$ is played as follows. There are $\omega_{1}$ rounds; before beginning round $\gamma<\omega_{1}$ we have premice $\mathscr{M}_{\beta}$, for $\beta \leq \gamma$, and embeddings $i_{\alpha \beta}: \mathscr{M}_{\alpha} \rightarrow \mathscr{M}_{\beta}$ for $\alpha<\beta$ which commute with one another. If $\gamma=0$, then $\mathscr{M}_{\gamma}=\mathscr{M}_{\text {, and }}$ if $\gamma$ is a limit, then $\mathscr{M}_{\gamma}$ is the direct limit of the $\mathscr{M}_{\alpha}$, for $\alpha<\gamma$, under the $i_{\alpha \beta}$ 's; player II loses if this direct limit is illfounded. Player I begins round $\gamma$ by playing a countable putative normal iteration tree on $\mathscr{M}_{\gamma}$. Player II can either accept $\mathscr{T}$ or play a maximal wellfounded branch of $\mathscr{T}$, with the proviso that he cannot accept $\mathscr{T}$ unless it actually is a normal tree of successor length (i.e., has a last, wellfounded, model). If II plays the maximal wellfounded branch $b$ of $\mathscr{T}$, then we set $\mathscr{M}_{\gamma+1}=\left(\mathscr{M}_{\gamma}\right)_{b}^{\mathscr{T}}$ and $i_{\gamma, \gamma+1}=i_{0 b}^{\mathscr{G}}$ and go on to round $\gamma+1$. If II accepts $\mathscr{T}$, then letting $\left(\mathscr{M}_{\gamma}\right)_{\alpha}^{\mathscr{T}}$ be the last model of $\mathscr{T}$ we set $\mathscr{M}_{\gamma+1}=\left(\mathscr{M}_{\gamma}\right)_{\alpha}^{\mathscr{T}}$ and $i_{\gamma, \gamma+1}=i_{0 \alpha}^{\mathscr{G}}$ and go on to round $\gamma+1$. The first player to break the rules loses $\mathscr{W} G^{*}\left(\mathscr{M}, \omega_{1}\right)$; if neither player breaks the rules, then II wins. We say $\mathscr{M}$ is weakly $\omega_{1}$-iterable just in case II has a winning strategy in $\mathscr{W} G^{*}\left(\mathscr{M}, \omega_{1}\right)$.

Corollary 5.12. Let $\mathscr{M}$ be a countable premouse such that $\mathscr{M} \preceq\left(V_{\alpha}, \in, \delta\right)$ for some $\alpha, \delta$. Then $\mathscr{M}$ is weakly $\omega_{1}$-iterable.

Proof. Let $\pi_{0}: \mathscr{M} \rightarrow\left(V_{\alpha}, \in, \delta\right)$ be elementary. Player II's winning strategy in $\mathscr{W} G^{*}\left(\mathscr{M}, \omega_{1}\right)$ is the following: letting $\mathscr{M}_{\gamma}$ be the model given at the beginning of round $\gamma$, and letting $i_{\beta \gamma}: \mathscr{M}_{\beta} \rightarrow \mathscr{M}_{\gamma}$ for $\beta<\gamma$ be the given embeddings, arrange that there is an elementary $\pi_{\gamma}: \mathscr{M}_{\gamma} \rightarrow\left(V_{\alpha}, \in, \delta\right)$ such that $\pi_{\beta}=$ $\pi_{\gamma} \circ i_{\beta \gamma}$ for all $\beta<\gamma$. From Theorem 4.3 we see that II can always arrange this, and clearly he wins by doing so.

It is easy to see that if $\mathscr{M}$ is normally $\omega_{1}$-iterable, then $\mathscr{M}$ is weakly $\omega_{1}$ iterable. The converse is certainly false, although we do not have a full proof of this. For premice $\mathscr{M}$ whose derived inner sequence $\vec{E}$ (see Definition 6.1) is such that $L[\vec{E}]$ does not have any Woodin cardinals $\delta$ such that there is a $(\kappa, \lambda)$ extender $E$ on $\vec{E}$ with $\kappa \leq \delta \leq \lambda$, we can show that weak $\omega_{1}$ iterability implies normal $\omega_{1}$-iterability. This fact and Corollary 5.12 lead to an inner model theory for large cardinal hypotheses weaker than the existence of a cardinal $\kappa$ which is $\delta+1$-strong, for some Woodin cardinal $\delta>\kappa$.

\section{ONE WOODIN CARDINAL}

In order to avoid some problems connected with the Axiom of Choice, we shall consruct our inner models not from sequences of extenders but from sequences of classes of extenders. Such objects are known as Doddages, although, as far as we know, Mitchell discovered them and established their basic properties. (See [Mi3].) The sort of Doddage we shall study, which is formally defined in Definition 6.1, was first isolated in [St1]. From that paper we also take the idea of a comparison process involving mice which approximate not just our inner model but $V$ itself. 
Recall that if $F$ is a $(\kappa, \lambda)$ extender, then $\kappa=\operatorname{crit}(F)$ is the critical point of $F$ and $\lambda=\ln (F)$ is the length of $F$. For $\kappa<\eta \leq \lambda$, we let $F \mid \eta=\left\langle F_{a} \mid a \in[\eta]^{<\omega}\right\rangle$. Now suppose we are given a sequence $(\mathscr{F}, \vec{\nu})=$ $\left\langle\left(\mathscr{F}_{\beta}, \nu_{\beta}\right) \mid \beta<\lambda\right\rangle$ such that each $\mathscr{F}_{\beta}$ is a family of extenders all of which have critical point $<\nu_{\beta}$ and length $>\nu_{\beta}$. For $\beta<\lambda$, let $\mathscr{E}_{\beta}=\left\{F\left|\nu_{\beta}\right| F \in \mathscr{F}_{\beta}\right\}$; we call the sequence $\mathscr{E}=\left\langle\mathscr{E}_{\beta} \mid \beta<\lambda\right\rangle$ the projection of $(\mathscr{F}, \vec{\nu})$. Let also $E_{\beta}=\bigcup \mathscr{E}_{\beta}=\left\{(a, x) \mid \exists E \in \mathscr{E}_{\beta}\left(x \in E_{a}\right)\right\}$ for all $\beta<\lambda$. We call $\left\langle E_{\beta} \mid \beta<\lambda\right\rangle$ the inner sequence derived from $(\mathscr{F}, \vec{\nu})$. By $L\left[\left\langle E_{\beta} \mid \beta<\gamma\right\rangle\right]$ we mean $L[A]$, where $A=\left\{(\beta, a, x) \mid \beta<\gamma\right.$ and $\left.(a, x) \in E_{\beta}\right\}$. Thus $L\left[\left\langle E_{\beta} \mid \beta<\gamma\right\rangle\right]$ is the smallest proper class $M$ satisfying ZFC such that $\left\langle E_{\beta} \cap M \mid \beta<\gamma\right\rangle \in M$.

Definition 6.1. A Doddage is a sequence $(\mathscr{F}, \vec{\nu})=\left\langle\left(\mathscr{F}_{\beta}, \nu_{\beta}\right) \mid \beta<\lambda\right\rangle$ such that each $\mathscr{F}_{\beta}$ is a class of extenders all of which have critical point $<\nu_{\beta}$ and length $>\nu_{\beta}$ and such that letting $\mathscr{E}$ be the projection of $(\mathscr{F}, \vec{\nu})$ and $\left\langle E_{\beta} \mid \beta<\lambda\right\rangle$ the derived inner sequence:

(1) $\alpha<\beta \Rightarrow \nu_{\alpha} \leq \nu_{\beta}$;

(2) $F \in \mathscr{F}_{\beta} \Rightarrow \operatorname{strength}(F) \geq \nu_{\beta}+2$;

(3) (Novelty) if $\alpha<\beta<\lambda$ and $\nu_{\alpha}=\nu_{\beta}$ and $F \in \mathscr{F}_{\beta}$ and $G \in \mathscr{F}_{\alpha}$, then $F$ and $G$ disagree over $L\left[\left\langle E_{\gamma} \mid \gamma<\beta\right\rangle\right]$; that is, there are $a \in\left[\nu_{\alpha}\right]^{<\omega}$ and $x \in L\left[\left\langle E_{\gamma} \mid \gamma<\beta\right\rangle\right]$ such that $x \in F_{a} \Delta G_{a}$;

(4) (External coherence) if $F \in \mathscr{F}_{\beta}$ and $i: V \rightarrow \operatorname{Ult}(V, F)$ is the canonical embedding, then

$$
i(\mathscr{E}) \uparrow \text { length } \leq \nu_{\beta}=\mathscr{E} \uparrow \beta
$$

where by $\mathscr{G} \uparrow$ length $\leq \nu$ we mean $\mathscr{G} \uparrow \alpha$, where $\alpha$ is least such that $\alpha=$ $\operatorname{dom}(\mathscr{G})$ or $\exists G \in \mathscr{G}_{\alpha}(\nu<\operatorname{lh}(G))$; and

(5) (Closure under initial segment) whenever $F \in \mathscr{E}_{\beta}$ and $\operatorname{crit}(F)<\mu<\nu_{\beta}$, there is an $\alpha<\beta$ such that $\nu_{\alpha}=\mu$ and a $G \in \mathscr{E}_{\alpha}$ such that $F \uparrow \mu$ agrees with $G$ over $L\left[\left\langle E_{\gamma} \mid \nu_{\gamma} \leq \mu\right\rangle\right]$, that is, such that for all $a \in[\mu]^{<\omega}$ and $x \in L\left[\left\langle E_{\gamma} \mid \nu_{\gamma} \leq \mu\right\rangle\right], x \in F_{a} \Leftrightarrow x \in G_{a}$.

Notice that any initial segment of a Doddage is a Doddage. There is a canonical maximal Doddage, which one constructs by adding all extenders possibie while maintaining inductively conditions (1)-(4); condition (5) then follows.

Definition 6.2. The maximal Doddage is defined by induction on $\beta \in \mathrm{OR}$ as follows. Suppose $\left\langle\left(\mathscr{F}_{\alpha}, \nu_{\alpha}\right) \mid \alpha<\beta\right\rangle$ has been defined, and let $\left\langle\mathscr{E}_{\alpha} \mid \alpha<\beta\right\rangle$ be its projection and $\left\langle E_{\alpha} \mid \alpha<\beta\right\rangle$ its derived inner sequence. Let $\nu \in \mathrm{OR}$ be least such that

(i) $\nu_{\alpha} \leq \nu$ for all $\alpha<\beta$, and for some extender $F$ such that $\operatorname{crit}(F)<\nu$;

(ii) $\operatorname{strength}(\mathrm{F}) \geq \nu+2$;

(iii) for any $\alpha<\beta$ such that $\nu_{\alpha}=\nu$ and any $G \in \mathscr{F}_{\alpha}$, there is an $a \in[\nu]^{<\omega}$ and $x \in L\left[\left\langle E_{\gamma} \mid \gamma<\beta\right\rangle\right]$ such that $x \in F_{a} \Delta G_{a}$; and

(iv) letting $i: V \rightarrow \operatorname{Ult}(V, F)$ be the canonical embedding,

$$
i\left(\left\langle\mathscr{E}_{\alpha} \mid \alpha<\beta\right\rangle\right) \uparrow(\text { length } \leq \nu)=\left\langle\mathscr{E}_{\alpha} \mid \alpha<\beta\right\rangle .
$$


Then we set $\nu_{\beta}=\nu$. Also, letting $\gamma$ be least such that some $F \in V_{\gamma}$ satisfies (ii), (iii), and (iv), we set

$$
\mathscr{F}_{\beta}=\left\{F \in V_{\gamma} \mid F \text { satisfies (ii), (iii), and (iv) }\right\} \text {. }
$$

If there is no such ordinal $\nu$, then $\left(\mathscr{F}_{\beta}, \nu_{\beta}\right)$ is undefined, and $\left\langle\left(\mathscr{F}_{\alpha}, \nu_{\alpha}\right)\right|$ $\alpha<\beta\rangle$ is the maximal Doddage.

If $\left(\mathscr{F}_{\alpha}, \nu_{\alpha}\right)$ is defined for all $\alpha \in \mathrm{OR}$, then $\left\langle\left(\mathscr{F}_{\alpha}, \nu_{\alpha}\right) \mid \alpha \in \mathrm{OR}\right\rangle$ is the maximal Doddage.

Lemma 6.3. The maximal Doddage is a Doddage.

Proof. We must check condition (5). Let $F \in \mathscr{F}_{\beta}$ and $\operatorname{crit}(F)<\mu<\nu_{\beta}$. Let $\gamma \leq \beta$ be at least such that $\mu<\nu_{\gamma}$. If at stage $\gamma$ in our construction we set $\nu=\mu$, then (i) is satisfied, and $F$ witnesses the satisfaction of (ii) and (iv) as $\mu<\nu_{\beta}$. Since $\nu_{\gamma}$ was chosen minimal, condition (iii) must fail for $\mu$ and $F$. That is, there is an $\alpha<\gamma$ and $G \in \mathscr{F}_{\alpha}$ such that $\nu_{\alpha}=\mu$ and $G \mid \nu_{\alpha}$ agrees with $F \mid \nu_{\alpha}$ over $L\left[\left\langle E_{\eta}\right| \eta\langle\gamma\rangle\right]=L\left[\left\langle E_{\eta} \mid \nu_{\eta} \leq \mu\right\rangle\right]$.

We now prove a comparison lemma for the inner sequences derived from Doddages.

Definition 6.4. An expanded premouse is a structure $(M, \in, \delta, \mathscr{F}, \vec{\nu})$ such that

(1) $(M, \in, \delta)$ is a premouse,

(2) $(M, \in) \models$ " $(\mathscr{F}, \vec{\nu})$ is a Doddage", and

(3) $\mathscr{F} \uparrow \eta \in V_{\delta}^{M}$, for all $\eta \in \operatorname{dom}(\mathscr{F})(\operatorname{sod} \operatorname{dom}(\mathscr{F}) \leq \delta)$.

An iteration tree on an expanded premouse $(M, \in, \delta, \mathscr{F}, \vec{\nu})$ is just an iteration tree $\mathscr{T}$ on the premouse $(M, \in, \delta)$ with its $\alpha$ th model expanded by $i_{0, \alpha}^{\mathscr{T}}((\mathscr{F}, \vec{\nu}))$.

Definition 6.5. An expanded premouse $(M, \in, \delta, \mathscr{F}, \vec{\nu})$ is (normally) $\theta$ iterable just in case $(M, \in, \delta)$ is (normally) $\theta$-iterable.

Definition 6.6. Let $\mathscr{M}=(M, \in, \delta, \mathscr{F}, \vec{\nu})$ and $\mathscr{N}=(N, \in, \gamma, \mathscr{G}, \vec{\rho})$ be expanded premice. Then we say $\mathscr{M}$ is an initial segment of $\mathscr{N}$ iff $\mathrm{OR}^{\mathscr{M}} \leq$ $\mathrm{OR}^{\mathscr{N}}$ and

(1) $\operatorname{dom}(\mathscr{F}) \leq \operatorname{dom}(\mathscr{G})$, and $\nu_{\beta}=\rho_{\beta}$ for all $\beta<\operatorname{dom}(\mathscr{F})$; and

(2) if $\beta \in \operatorname{dom}(\mathscr{F}), F \in \mathscr{F}_{\beta}$, and $G \in \mathscr{G}_{\beta}$, then $F \uparrow \nu_{\beta}$ agrees with $G \backslash \rho_{\beta}$ over $M \cap N$.

We now prove a comparison lemma for "sufficiently iterable" expanded premice. Versions of this lemma, and even more, of the comparison process on which its proof is based, constitute the basic tool in virtually all work in inner model theory. We shall state a general comparison lemma which should some day apply to inner models with superstrong cardinals. (We cannot so apply it now because we cannot yet show that the expanded premice which arise in the theory of inner models with superstrongs are "sufficiently iterable".)

Lemma 6.7 (The Comparison lemma). Let $\mathscr{M}$ and $\mathscr{N}$ be normally $(\theta+1)$ iterable expanded premice, where $\theta=\max (\operatorname{card}(\mathscr{M}), \operatorname{card}(\mathscr{N}))^{+}$. Then there 
are iteration trees $\mathscr{T}$ on $\mathscr{M}$, with last model $\mathscr{P}$, and $\mathscr{U}$ on $\mathscr{N}$, with last model $\mathscr{Q}$, such that either $\mathscr{P}$ is an initial segment of $\mathscr{Q}$ or $\mathscr{Q}$ is an initial segment of $\mathscr{P}$.

Proof. Fix winning strategies $\Gamma$ and $\Sigma$ for II in the length $\theta+1$ normal iteration games on $\mathscr{M}$ and $\mathscr{N}$ respectively. We define by induction on $\alpha$ iteration trees $\mathscr{T}_{\alpha}$ on $\mathscr{M}$ and $\mathscr{U}_{\alpha}$ on $\mathscr{N}$. We shall have

(a) $\alpha<\beta \Rightarrow\left(\mathscr{T}_{\alpha}=\mathscr{T}_{\beta} \mid \ln \left(\mathscr{T}_{\alpha}\right)\right.$ and $\mathscr{U}_{\alpha}=\mathscr{U}_{\beta}\left\lceil\ln \left(\mathscr{U}_{\alpha}\right)\right)$, where if $\mathscr{T}=\left\langle\mathrm{T},\left\langle E_{\gamma} \mid \gamma+1<\theta\right\rangle\right\rangle$ is an iteration tree, then $\mathscr{T} \mid \eta=$ $\left\langle\mathrm{T} \cap(\eta \times \eta),\left\langle E_{\gamma} \mid \gamma+1<\eta\right\rangle\right\rangle$.

We shall have $\mathscr{T}_{\alpha}=\mathscr{T}_{\alpha+1}$ or $\operatorname{lh}\left(\mathscr{T}_{\alpha+1}\right)=\operatorname{lh}\left(\mathscr{T}_{\alpha}\right)+1$, and similarly for $\mathscr{U}_{\alpha}$. Moreover, $\operatorname{lh}\left(\mathscr{T}_{\alpha}\right)$ and $\operatorname{lh}\left(\mathscr{U}_{\alpha}\right)$ will be successor ordinals, so that $\mathscr{T}_{\alpha}$ and $\mathscr{U}_{\alpha}$ have last models, for all $\alpha$. (It will be possible that $\operatorname{lh}\left(\mathscr{U}_{\alpha}\right) \neq \operatorname{lh}\left(\mathscr{T}_{\alpha}\right)$.)

We shall also maintain by induction

(b) $\mathscr{T}_{\alpha}$ is a play according to $\Gamma$ in which $I$ has not yet lost, and $\mathscr{U}_{\alpha}$ is a play according to $\Sigma$ in which I has not yet lost.

Thus, in order to determine $\mathscr{T}_{\alpha+1}$ and $\mathscr{U}_{\alpha+1}$, we need a way for I to play in the iteration games on $\mathscr{M}$ and $\mathscr{N}$. We shall have I extend $\mathscr{T}_{\alpha}$ to $\mathscr{T}_{\alpha+1}$ and $\mathscr{U}_{\alpha}$ to $\mathscr{U}_{\alpha+1}$ by "iterating the least disagreement" between the last models of $\mathscr{T}_{\alpha}^{\alpha}$ and $\mathscr{\mathscr { U }}_{\alpha}^{\alpha+}$. (This idea goes back to [Mi1].)

Suppose $\mathscr{P}=(P, \in, \delta, \mathscr{F}, \vec{\mu})$ and $\mathscr{Q}=(Q, \in, \eta, \mathscr{G}, \vec{\nu})$ are expanded premice, and let $\beta \in \operatorname{dom}(\mathscr{F}) \cap \operatorname{dom}(\mathscr{G})$ be least such that either $\mu_{\beta} \neq \nu_{\beta}$ or there are $F \in \mathscr{F}_{\beta}$ and $G \in \mathscr{G}_{\beta}$ such that $F \mid \mu_{\beta}$ disagrees with $G \nmid \nu_{\beta}$ over $P \cap Q$. We say in this case that $\beta$ indexes a least disagreement between $\mathscr{P}$ and $\mathscr{Q}$. We say that $(F, G)$ is a least disagreement of $\mathscr{P}$ with Q iff

(i) $\mu_{\beta}<\nu_{\beta}, F \in \mathscr{F}_{\beta}$, and $G=\varnothing$; or

(ii) $\nu_{\beta}<\mu_{\beta}, G \in \mathscr{G}_{\beta}$, and $F=\varnothing$; or

(iii) $\mu_{\beta}=\nu_{\beta}, F \in \mathscr{F}_{\beta}, G \in \mathscr{G}_{\beta}$ and $F \mid \mu_{\beta}$ disagrees with $G \uparrow \nu_{\beta}$ over $P \cap Q$.

Suppose that we have defined $\mathscr{T}_{\alpha}$ and $\mathscr{U}_{\alpha}$ and that they have last models $\mathscr{P}=(P, \ldots, \vec{\mu})$ and $\mathscr{Q}=(Q, \ldots, \vec{\nu})$ respectively. Then we set $\rho_{\alpha}=$ $\inf \left(\mu_{\beta}, \nu_{\beta}\right)$, where $\beta$ indexes a least disagreement between $\mathscr{P}$ and $\mathscr{Q}$. We shall maintain inductively

(c) $\alpha<\beta \Rightarrow \rho_{\alpha}<\rho_{\beta}$

(d) if $\mathscr{P}$ is the last model of $\mathscr{T}_{\alpha}, \alpha<\beta$, and $\mathscr{Q}$ is the last model of $\mathscr{T}_{\beta}$, then $\mathscr{P}$ and $\mathscr{Q}$ agree below $\rho_{\alpha}+2$ (that is, $V_{\rho+2}^{\mathscr{D}}=V_{\rho+2}^{\mathscr{Q}}$, where $\rho=$ $\rho_{\alpha}$ ). Moreover, if $\mathscr{E}$ and $\mathscr{H}$ are the projections of the Doddages of $\mathscr{P}$ and $\mathscr{Q}^{\alpha}$ respectively, then $\mathscr{E}|\gamma=\mathscr{H}|$ length $\leq \rho_{\alpha}$, where $\gamma$ indexes a least disagreement between $\mathscr{P}$ and the last model of $\mathscr{U}_{\alpha}$;

(e) if $\mathscr{P}$ is the last model of $\mathscr{U}_{\alpha}, \alpha<\beta$, and $Q$ is the last model of $\mathscr{U}_{\beta}$, then $\mathscr{P}$ and $\mathscr{Q}$ agree below $\rho_{\alpha}+2$. Moreover, if $\mathscr{E}$ and $\mathscr{H}$ are the projections of the Doddages of $\mathscr{P}$ and $\mathscr{Q}$ respectively, then $\mathscr{E}|\gamma=\mathscr{H}|$ length $\leq \rho_{\alpha}$, where $\gamma$ indexes a least disagreement between $\mathscr{P}$ and the last model of $\mathscr{T}_{\alpha}$.

We proceed to the construction of $\mathscr{T}$ and $\mathscr{U}$. Let $\mathscr{T}_{0}$ and $\mathscr{U}_{0}$ be the unique 
length 1 iteration trees on $\mathscr{M}$ and $\mathscr{N}$ respectively. Now suppose $\mathscr{T}_{\alpha}$ and $\mathscr{U}_{\alpha}$ are given; say $\mathscr{T}_{\alpha}=\left\langle\mathrm{T},\left\langle F_{\xi} \mid \xi+1<\gamma\right\rangle\right\rangle$ and $\mathscr{U}_{\alpha}=\left\langle\mathrm{U},\left\langle G_{\xi} \mid \xi+1<\delta\right\rangle\right\rangle$. Fix $(F, G)$ such that $(F, G)$ is a least disagreement between the last models of $\mathscr{T}_{\alpha}$ and $\mathscr{U}_{\alpha}$. If $F=\varnothing$, then we let $\mathscr{T}_{\alpha+1}=\mathscr{T}_{\alpha}$. Otherwise let $\beta=$ least $\eta<\alpha$ such that $\mathscr{T}_{\eta} \neq \mathscr{T}_{\eta+1}$ and $\operatorname{crit}(F) \stackrel{\alpha+1}{\leq} \rho_{\eta}$, or $\beta=\alpha$, if no such $\eta$ exists. Let $\xi+1=\ln \left(\mathscr{T}_{\beta}\right)$, so that $\mathscr{M}_{\xi}^{\mathscr{I}_{\beta}}=\mathscr{M}_{\xi}^{\mathscr{F}_{\alpha}}$ is the last model of $\mathscr{T}_{\beta}$. Since $\operatorname{crit}(F) \leq \rho_{\beta}$, induction hypothesis (d) implies that if I extends $\mathscr{T}_{\alpha}$ by applying $F$ to $\mathscr{M}_{\xi}^{\mathscr{F}_{\alpha}}$, he violates no rules of the iteration game. So we have I do this; that is, we let $\mathscr{T}_{\alpha+1}=\left\langle\mathrm{T}^{\prime},\left\langle F_{\eta} \mid \eta+1<\gamma\right\rangle\langle\langle F\rangle\rangle\right.$, where $\mathrm{T}^{\prime}=\mathrm{T} \cup\{(\eta, \gamma) \mid$ $\eta \mathrm{T} \xi$ or $\eta=\xi\}$.

We extend $\mathscr{U}_{\alpha}$ to $\mathscr{U}_{\alpha+1}$ similarly. If $G=\varnothing$, set $\mathscr{U}_{\alpha+1}=\mathscr{U}_{\alpha}$. Otherwise, let $\sigma$ be the least $\eta<\alpha$ such that $\mathscr{U}_{\eta} \neq \mathscr{U}_{\eta+1}$ and $\operatorname{crit}(G) \leq \rho_{\eta}$, or let $\sigma=\alpha$ if no such $\eta$ exists. Let $\tau+1=\operatorname{lh}\left(\mathscr{U}_{\sigma}\right)$. Since $\operatorname{crit}(G) \leq \rho_{\sigma}$, induction hypothesis (e) implies that if I extends $\mathscr{U}_{\alpha}$ by applying $G$ to the last model of $\mathscr{U}_{\sigma}$, he violates no rules of the iteration game. So we have I do this; that is, we set $\mathscr{U}_{\alpha+1}=\left\langle\mathrm{U}^{\prime},\left\langle G_{\eta} \mid \eta+1<\delta\right\rangle^{\curlyvee}\langle G\rangle\right\rangle$, where $\mathrm{U}^{\prime}=\mathrm{U} \cup\{(\eta, \delta) \mid \eta \mathrm{U} \tau$ or $\eta=\tau\}$.

Let us check that our induction hypotheses (a)-(e) still hold. For (a) this is clear, and we have already checked (b). (Notice that (b) implies that $\mathscr{T}_{\alpha+1}$ and $\mathscr{U}_{\alpha+1}$ are indeed iteration trees; their last models are wellfounded.) Induction hypotheses (c)-(e) follow at once from the next claim. If $\mathscr{P}$ is an expanded premouse, let us write $\mathscr{E}^{\mathscr{P}}$ for the projection of the Doddage of $\mathscr{P}$. Let now $\mathscr{P}$ and $\mathscr{Q}$ be the last models of $\mathscr{T}_{\alpha}$ and $\mathscr{U}_{\alpha}$ and $\mathscr{R}$ and $\mathscr{S}$ the last models of $\mathscr{T}_{\alpha+1}$ and $\mathscr{U}_{\alpha+1}$. Let $\rho=\rho_{\alpha}$, and let $\eta$ index the the least disagreement between $\mathscr{P}$ and $\mathscr{Q}$.

Claim. (1) $V_{\rho+2}^{\mathscr{R}}=V_{\rho+2}^{\mathscr{P}}$, and $\mathscr{E}^{\mathscr{P}}\left|\eta=\mathscr{E}^{\mathscr{R}}\right|$ (length $\leq \rho$ ).

(2) $V_{\rho+2}^{\mathscr{Q}}=V_{\rho+2}^{\mathscr{S}}$, and $\mathscr{E}^{\mathscr{Q}} \uparrow \eta=\mathscr{E}^{S} \uparrow$ (length $\leq \rho$ ).

Proof. We prove (1); the proof of (2) is the same. If $\mathscr{T}_{\alpha+1}=\mathscr{T}_{\alpha}$, the claim is obvious, so let $\mathscr{T}_{\alpha+1} \neq \mathscr{T}_{\alpha}$. Let $\mathscr{R}=\operatorname{Ult}(\mathscr{L}, F)$, where $\mathscr{L}=\mathscr{M}_{\xi}^{\mathscr{T} \alpha}$ is as in the definition of $\mathscr{T}_{\alpha+1}$. Since $\mathscr{L}$ agrees with $\mathscr{P}$ below $\operatorname{crit}(F)$ and since strength $^{\mathscr{P}}(F) \geq \rho+2, \mathscr{P}$ agrees with $\mathscr{R}$ below $\rho+2$. (See Lemma 1.5.) The same proof shows the second clause of $(1)$. Let $\mathscr{R}^{\prime}=\operatorname{Ult}(\mathscr{P}, F)$. By external coherence, $\mathscr{E}^{P}\left|\eta=\mathscr{E}^{\mathscr{R}^{\prime}}\right|($ length $\leq \rho)$. But $\mathscr{E}^{P} \mid($ length $<\operatorname{crit}(F))=$ $\mathscr{E}^{L} \uparrow($ length $<\operatorname{crit}(F))$, so $\mathscr{E}^{\mathscr{R}^{\prime}} \uparrow($ length $<i(\operatorname{crit}(F)))=\mathscr{E}^{R} \uparrow$ (length $<$ $i(\operatorname{crit}(F)))$, where $i: \mathscr{L} \rightarrow \mathscr{R}$ is the canonical embedding. As $i(\operatorname{crit}(F))>\rho$, the desired conclusion follows.

The claim easily implies $\rho_{\alpha}<\rho_{\alpha+1}$, which is what we need for (c). Induction hypotheses (d) and (e) also follow easily from the claim.

We proceed to the limit step in the inductive definitions of $\mathscr{T}$ and $\mathscr{U}$. Suppose we have constructed $\mathscr{T}_{\alpha}$ and $\mathscr{U}_{\alpha}$ for $\alpha<\lambda$, where $\lambda$ is a limit, and that (a)-(e) hold at $\alpha<\lambda$. Let $\mathscr{T}^{\prime}=\bigcup_{\alpha<\lambda} \mathscr{T}_{\alpha}$ and $\mathscr{U}^{\prime}=\bigcup_{\alpha<\lambda} \mathscr{U}_{\alpha}$. If $\gamma=\ln \left(\mathscr{T}^{\prime}\right)$ is a successor ordinal, then $\mathscr{T}^{\prime}=\mathscr{T}_{\alpha}$ for some $\alpha<\lambda$, and in this 
case we set $\mathscr{T}_{\lambda}=\mathscr{T}^{\prime}$. Otherwise, $\gamma$ is a limit ordinal. By (b), there is a unique iteration tree of length $\gamma+1$ which extends $\mathscr{T}^{\prime}$ and is a play according to $\Gamma$ in the iteration game on $\mathscr{M}$. We let $\mathscr{T}_{\lambda}$ be this iteration tree. Similarly, if $\delta=\ln \left(\mathscr{U}^{\prime}\right)$ is a successor, then $\mathscr{U}_{\lambda}=\mathscr{U}^{\prime}$, and otherwise $\mathscr{U}_{\lambda}$ results from letting $\Sigma$ choose a cofinal wellfounded branch of $\mathscr{U}^{\prime}$.

We leave it to the reader to check (a)-(e) at stage $\lambda$.

If we ever reach a stage $\alpha<\theta$ such that the last models of $\mathscr{T}_{\alpha}$ and $\mathscr{U}_{\alpha}$ have no least disagreement (that is, one is an initial segment of the other), then we stop the construction and set $\mathscr{T}=\mathscr{T}_{\alpha}$ and $\mathscr{U}=\mathscr{U}_{\alpha}$. The pair $(\mathscr{T}, \mathscr{U})$ witnesses the truth of Lemma 6.7. So it is enough to show

Claim. The construction stops at some $\alpha<\theta$.

Proof. Assume otherwise, and let $\mathscr{T}=\mathscr{T}_{\theta}$ and $\mathscr{U}=\mathscr{U}_{\theta}$. Since $\theta$ is regular and $\max (\operatorname{card}(\mathscr{M}), \operatorname{card}(\mathscr{N}))<\theta$, we must have $\ln (\mathscr{T})=\operatorname{lh}(\mathscr{U})=\theta+1$. Let $\mathrm{T}$ and $\mathrm{U}$ be the tree orderings of $\mathscr{T}$ and $\mathscr{U}$.

The following subclaim expresses the fact that "generators are not moved" along the branches of $\mathscr{T}$ and $\mathscr{U}$. This is crucial in showing that the construction stops.

Subclaim. (a) Let $(\alpha+1) \mathrm{T} \gamma$ and $\beta=\mathrm{T}-\operatorname{pred}(\alpha+1)$. Suppose $\mathscr{M}_{\alpha}^{\mathscr{T}}$ is the last model of $\mathscr{T}_{\xi}$, where $\mathscr{T}_{\xi} \neq \mathscr{T}_{\xi+1}$. Then $\operatorname{crit}\left(i_{\alpha+1, \gamma}^{\mathscr{T}}\right)>\rho_{\xi}$.

(b) Similarly for $\mathscr{U}$.

Proof. We prove (a). If (a) fails, then we have $\delta$ such that $(\alpha+1) \mathrm{T}$ $(\delta+1),(\delta+1) \mathrm{T} \gamma$ or $\delta+1=\gamma$, and $\operatorname{crit}\left(E_{\delta}^{\mathscr{T}}\right) \leq \rho_{\xi}$. By the construction of $\mathscr{T}, \mathrm{T}-\operatorname{pred}(\delta+1)=\eta$, where $\mathscr{M}_{\eta}^{\mathscr{T}}$ is the last model of some $\mathscr{T}_{\beta}$, for $\beta \leq \xi$. But then T-pred $(\delta+1) \leq \alpha$, which contradicts the fact that $(\alpha+1) \mathrm{T}(\delta+1)$.

Let $b=\{\alpha \mid \alpha \mathrm{T} \theta\}$ and $c=\{\alpha \mid \alpha \mathrm{U} \theta\}$. Since every branch of an iteration tree is closed below its sup, $b$ and $c$ are club in $\theta$. Using arguments from [Mil], we can find a set $D \subseteq b \cap c$ which is club in $\theta$ and such that for $\alpha, \beta \in D$ with $\alpha<\beta$ :

(i) $\operatorname{crit}\left(i_{\alpha, \theta}^{\mathscr{G}}\right)=\operatorname{crit}\left(i_{\alpha, \theta}^{\mathscr{U}}\right)=\alpha$,

(ii) $i_{\alpha, \beta}^{\mathscr{I}}(\alpha)=i_{\alpha, \beta}^{\mathscr{U}}(\alpha)=\beta$,

(iii) for all $A \subseteq \alpha$ such that $A \in \mathscr{M}_{\alpha}^{\mathscr{T}} \cap \mathscr{M}_{\alpha}^{\mathscr{U}}, i_{\alpha, \beta}^{\mathscr{T}}(A)=i_{\alpha, \beta}^{\mathscr{U}}(A)$.

[Proof. For $\lambda$ a limit ordinal in $b \cap c$, let $f(\lambda)=$ least $\beta \mathrm{T} \lambda$ such that $\operatorname{crit}\left(i_{\lambda, \theta}^{\mathscr{T}}\right) \in \operatorname{ran}\left(i_{\beta, \lambda}^{\mathscr{T}}\right)$. There is a stationary set $S \subseteq b \cap c$ such that $f$ is constant on $S$, with constant value $\beta$. As $\mathscr{M}_{\beta}^{\mathscr{T}}$ has cardinality $<\theta$, we can shrink $S$ so that there is a fixed $\kappa \in \mathscr{M}_{\beta}^{\mathscr{T}}$ such that for all $\lambda \in S, \operatorname{crit}\left(i_{\lambda, \theta}^{\mathscr{T}}\right)=i_{\beta, \lambda}^{\mathscr{T}}(\kappa)$. Let

$$
D_{1}=\left\{\lambda \in b \cap c \mid \beta<\lambda \wedge \operatorname{crit}\left(i_{\lambda, \theta}^{\mathscr{T}}\right)=i_{\beta, \lambda}^{\mathscr{G}}(\kappa)\right\} .
$$

Now our Subclaim guarantees that if $\eta \mathrm{T} \xi \mathrm{T} \delta$, then $\operatorname{crit}\left(i_{\eta, \delta}^{\mathscr{G}}\right)<\operatorname{crit}\left(i_{\xi, \delta}^{\mathscr{G}}\right)$. It follows easily from this that $D_{1}$ is closed in $\theta$ and, hence, club in $\theta$. 
By a similar argument, we can find $\beta^{\prime} \in b \cap c$ and $\kappa^{\prime}$ such that, letting

$$
D_{2}=\left\{\lambda \in b \cap c \mid \beta^{\prime}<\lambda \wedge \operatorname{crit}\left(i_{\lambda, \theta}^{\mathscr{U}}\right)=i_{\beta^{\prime}, \lambda}^{\mathscr{U}}\left(\kappa^{\prime}\right)\right\},
$$

$D_{2}$ is club in $\theta$. Set

$$
D_{3}=\left\{\lambda \in D_{1} \cap D_{2} \mid i_{\beta, \lambda}^{\mathscr{T}}(\kappa)=\lambda=i_{\beta^{\prime}, \lambda}^{\mathscr{U}}\left(\kappa^{\prime}\right)\right\} .
$$

It is easy to see that $D_{3}$ is club and (i) and (ii) above hold for all $\alpha, \beta \in D_{3}$ with $\alpha<\beta$.

Let

$$
\begin{array}{r}
\alpha \in S \Leftrightarrow \alpha \in D_{3} \wedge \exists A \in\left(P(\alpha) \cap \mathscr{M}_{\alpha}^{\mathscr{T}} \cap \mathscr{M}_{\alpha}^{\mathscr{U}}\right) \\
{\left[\exists \beta \in D_{3}\left(\alpha<\beta \wedge i_{\alpha \beta}^{\mathscr{g}}(A) \neq i_{\alpha \beta}^{\mathscr{U}}(A)\right)\right] .}
\end{array}
$$

It is enough to show $S$ is nonstationary, so assume otherwise. For $\alpha \in S$, let $A_{\alpha} \in\left(P(\alpha) \cap \mathscr{M}_{\alpha}^{\mathscr{T}} \cap \mathscr{M}_{\alpha}^{\mathscr{U}}\right)$ witness that $\alpha \in S$. As in the preceding paragraphs, we can find a stationary set $R \subseteq S$ such that

$$
(\alpha, \beta \in R \wedge \alpha<\beta) \Rightarrow i_{\alpha \beta}^{\mathscr{G}}\left(A_{\alpha}\right)=i_{\alpha \beta}^{\mathscr{U}}\left(A_{\alpha}\right)=A_{\beta} .
$$

But now fix $\alpha \in R$. Fix $\beta \in D_{3}$ such that $\alpha<\beta$ and $i_{\alpha \beta}^{\mathscr{T}}\left(A_{\alpha}\right) \neq i_{\alpha \beta}^{\mathscr{U}}\left(A_{\alpha}\right)$; such a $\beta$ exists by the definition of $S$. Let $\beta<\gamma$, where $\gamma \in R$. Then $i_{\alpha \gamma}^{\mathscr{G}}\left(A_{\alpha}\right)=i_{\alpha \gamma}^{\mathscr{U}}\left(A_{\alpha}\right)$, so $i_{\beta \gamma}^{\mathscr{G}}\left(i_{\alpha \beta}^{\mathscr{G}}\left(A_{\alpha}\right)\right)=i_{\beta \gamma}^{\mathscr{U}}\left(i_{\alpha \beta}^{\mathscr{U}}\left(A_{\alpha}\right)\right)$. As $\operatorname{crit}\left(i_{\beta \gamma}^{\mathscr{G}}\right)=\operatorname{crit}\left(i_{\beta \gamma}^{\mathscr{U}}\right)=\beta$ and $i_{\alpha \beta}^{\mathscr{G}}\left(A_{\alpha}\right)$ and $i_{\alpha \beta}^{\mathscr{U}}\left(A_{\alpha}\right)$ are subsets of $\beta$, this implies $i_{\alpha \beta}^{\mathscr{G}}\left(A_{\alpha}\right)=i_{\alpha \beta}^{\mathscr{U}}\left(A_{\alpha}\right)$, a contradiction.]

Now fix $\alpha \in D$. Let $\gamma+1 \in b$ be such that U-pred $(\gamma+1)=\alpha$ and $\delta+1 \in c$ be such that T-pred $(\delta+1)=\alpha$. Let $\xi$ be such that $\mathscr{M}_{\gamma}^{\mathscr{T}}$ is the last model of $\mathscr{T}_{\xi}$ and $\mathscr{T}_{\xi} \neq \mathscr{T}_{\xi+1}$. Let $\eta$ be such that $\mathscr{M}_{\delta}^{\mathscr{U}}$ is the last model of $\mathscr{U}_{\eta}$ and $\mathscr{U}_{\eta} \neq \mathscr{U}_{\eta+1}$. We assume without loss of generality that $\xi \leq \eta$. Let $F=E_{\gamma}^{\mathscr{G}}$ and $G=E_{\delta}^{\mathscr{U}}$, and let $\rho=\rho_{\xi}$.

We claim that $F\left\lceil\rho\right.$ agrees with $G \uparrow \rho$ over $\mathscr{M}_{\alpha}^{\mathscr{T}} \cap \mathscr{M}_{\alpha}^{\mathscr{U}}$. This follows from property (iii) of $D$. For let $\beta \in D$ and $\max \left(\gamma+1, \delta^{\alpha} \delta+1\right)<\beta$. Let $A \subseteq[\alpha]^{n}$ and $A \in \mathscr{M}_{\alpha}^{\mathscr{T}} \cap \mathscr{M}_{\alpha}^{\mathscr{U}}$. Since $i_{\alpha \beta}^{\mathscr{G}}(A)=i_{\alpha \beta}^{\mathscr{U}}(A)$ and $\operatorname{crit}\left(i_{\gamma+1, \beta}^{\mathscr{G}}\right)>\rho$ and $\operatorname{crit}\left(i_{\delta+1, \beta}^{\mathscr{U}}\right)>\rho, i_{\alpha, \gamma+1}^{\mathscr{J}}(A) \cap[\rho]^{n}=i_{\alpha, \delta+1}^{\mathscr{U}}(A) \cap[\rho]^{n}$. But then for $a \in[\rho]^{n}, A \in F_{a} \Leftrightarrow a \in i_{\alpha, \gamma+1}^{\mathscr{Y}}(A) \Leftrightarrow a \in i_{\alpha, \delta+1}^{\mathscr{U}}(A) \Leftrightarrow A \in G_{a}$, as desired.

Notice that $P(\alpha) \cap \mathscr{M}_{\alpha}^{\mathscr{T}}=P(\alpha) \cap \mathscr{M}_{\sigma}^{\mathscr{T}}$ for all $\sigma \geq \alpha$. For $\mathscr{M}_{\alpha}^{\mathscr{T}}$ is the last model of $\mathscr{T}$ for some $\nu \geq \alpha$, and $\rho_{\nu} \geq \alpha$ since the $\rho$ 's are increasing. Thus our induction hypothesis (d) gives the desired conclusion. Similarly, $P(\alpha) \cap$ $\mathscr{M}_{\alpha}^{\mathscr{U}}=P(\alpha) \cap \mathscr{M}_{\sigma}^{\mathscr{U}}$ for all $\sigma \geq \alpha$.

We claim that $\xi \neq \eta$. For otherwise, we see from the construction of $\mathscr{T}_{\xi+1}$ and $\mathscr{U}_{\xi+1}$ that $(F, G)$ is a least disagreement between $\mathscr{M}_{\gamma}^{\mathscr{T}}$ and $\mathscr{M}_{\delta}^{\mathscr{U}}$. This contradicts the fact that $F\left\lceil\rho\right.$ agrees with $G \uparrow \rho$ over $P(\alpha) \cap \mathscr{M}_{\gamma}^{\mathscr{T}} \cap \mathscr{M}_{\delta}^{\mathscr{U}}$. 
So $\xi<\eta$, and $\rho=\rho_{\xi}<\rho_{\eta}$. Let $(\mathscr{F}, \vec{\nu})$ be the Doddage of $\mathscr{M}_{\delta}^{\mathscr{U}}, \mathscr{E}$ its projection, and $\left\langle E_{\gamma} \mid \gamma<\operatorname{lh}(\mathscr{F})\right\rangle$ its derived inner sequence. By the initial segment condition on Doddages, there is an $H \in \mathscr{E}_{\beta}$, for some $\beta$ such that $\nu_{\beta}=\rho$, such that $G \uparrow \rho$ agrees with $H$ over $L\left[\left\langle E_{\gamma} \mid \nu_{\gamma} \leq \rho\right\rangle\right]^{\mathscr{\mu}_{\delta}^{\mathcal{U}}}$. Notice that $L\left[\left\langle E_{\gamma} \mid \nu_{\gamma} \leq \rho\right\rangle\right]^{\mathscr{M}_{\delta}^{\mathcal{L}}}$ and the full $L\left[\left\langle E_{\gamma} \mid \nu_{\gamma} \leq \rho\right\rangle\right]$ have the same subsets of $\rho$. [We show by induction on $\eta$ such that $\delta<\eta \leq \theta$ that $L\left[\left\langle E_{\gamma} \mid \nu_{\gamma} \leq \rho\right\rangle\right]^{\mathscr{M}_{\eta}^{\mathscr{L}}}$ and $L\left[\left\langle E_{\gamma} \mid \nu_{\gamma} \leq \rho\right\rangle\right]^{\mathscr{M}_{\delta}^{u}}$ have the same subsets of $\rho$, or, in other words, that if $A \subseteq \rho$ and $A \in L_{\xi}\left[\left\langle E_{\gamma} \mid \nu_{\gamma} \leq \rho\right\rangle\right]$ for some $\xi \in \mathrm{OR}^{\mathscr{M}_{\eta}^{\mathscr{L}}}$, then $A \in L_{\xi}\left[\left\langle E_{\gamma} \mid \nu_{\gamma} \leq \rho\right\rangle\right]$ for some $\xi \in \mathrm{OR}^{\mathscr{M}_{\delta}^{\mathscr{U}}}$. Suppose first $\eta$ is a successor. Let $\kappa=\operatorname{crit}\left(E_{\eta-1}^{\mathscr{U}}\right)$ and $\mu=\mathrm{U}-\operatorname{pred}(\eta)$. Then $i_{\mu \eta}^{\mathscr{U}}(\kappa)$ is a cardinal of $\mathscr{M}_{\eta}^{\mathscr{U}}$ and $\rho<i_{\mu \eta}^{\mathscr{U}}(\kappa)$, so all subsets of $\rho$ in $L\left[\left\langle E_{\gamma} \mid \nu_{\gamma} \leq \rho\right\rangle\right]^{\mathscr{K}_{\eta}^{\mathscr{U}}}$ are constructed before $i_{\mu \eta}^{\mathscr{U}}(\kappa)$. But $i_{\mu \eta}^{\mathscr{U}}(\kappa) \in \mathrm{OR}^{\mathscr{K}_{\eta-1}^{\mathscr{U}}}$, which finishes the successor case. We leave the case $\eta$ is a limit to the reader. Finally, since $\theta$ is a cardinal of $V, \theta \leq \mathrm{OR}^{\mathscr{K}_{\theta}^{\mathscr{U}}}$, and $\rho<\theta$, we have $P(\rho) \cap L\left[\left\langle E_{\gamma} \mid \nu_{\gamma} \leq \rho\right\rangle\right] \subseteq L\left[\left\langle E_{\gamma} \mid \nu_{\gamma} \leq \rho\right\rangle\right]^{\mathscr{K}_{\theta}^{\mathscr{U}}}$. $]$ Thus $G \uparrow \rho$ agrees with $H$ over the full $L\left[\left\langle E_{\gamma} \mid \nu_{\gamma} \leq \rho\right\rangle\right]$.

Let $\mathscr{P}$ and $\mathscr{Q}$ be the last models of $\mathscr{T}_{\xi}$ and $\mathscr{U}_{\xi}$ respectively. Let $\left(\mathscr{F}^{\prime}, \vec{\nu}^{\prime}\right)$ be the Doddage of $\mathscr{P}, \mathscr{E}^{\prime}$ its projection, and $\left\langle E_{\gamma}^{\prime} \mid \gamma<\operatorname{lh}\left(\mathscr{F}^{\prime}\right)\right\rangle$ its derived inner sequence. Let $\tau$ index a least disagreement between $\mathscr{P}$ and $\mathscr{Q}$. (Thus $\nu_{\tau}^{\prime}=\rho$ and $F \in \mathscr{F}_{\tau}^{\prime}$; moreover, $\nu_{\gamma} \leq \rho$ iff $\gamma<\tau$.) By our inductive hypothesis (e), $\beta<\tau$ (where $\beta$ is such that $H \in \mathscr{E}_{\beta}$ ) and $\mathscr{E}_{\beta}=\mathscr{E}_{\beta}^{\mathscr{Q}}$. As $\beta<\tau$, there is a $K \in \mathscr{E}_{\beta}^{\prime}$ such that $H$ agrees with $K$ over $\mathscr{P} \cap \mathscr{Q}$.

By (e), $\left\langle E_{\gamma} \mid \gamma<\tau\right\rangle=\left\langle E_{\gamma} \mid \nu_{\gamma} \leq \rho\right\rangle$ is an initial segment of the derived inner sequence of $\mathscr{Q}$. Moreover, $E_{\gamma}$ and $E_{\gamma}^{\prime}$ agree on all sets in $\mathscr{P} \cap \mathscr{Q}, \forall \gamma<\tau$. By the argument given two paragraphs back, $\mathscr{P}$ computes $P(\rho) \cap L\left[\left\langle E_{\gamma}^{\prime} \mid \gamma<\tau\right\rangle\right]$ correctly and $Q$ computes $P(\rho) \cap L\left[\left\langle E_{\gamma} \mid \gamma<\tau\right\rangle\right]$ correctly. It follows that $\forall \gamma<\tau, E_{\gamma}^{\prime}$ agrees with $E_{\gamma}$ over $L\left[\left\langle E_{\gamma}^{\prime} \mid \gamma<\tau\right\rangle\right]=L\left[\left\langle E_{\gamma} \mid \gamma<\tau\right\rangle\right]$.

But then $F \uparrow \rho$ agrees with $G \uparrow \rho$, hence with $H$, hence with $K$, over $L\left[\left\langle E_{\gamma}^{\prime} \mid \gamma<\tau\right\rangle\right]$. This contradicts the novelty condition on $\mathscr{F}^{\prime}$.

This contradiction completes the proof of the claim and thereby completes the proof of Lemma 6.7.

The next theorem shows that by setting $E_{\beta}=\bigcup \mathscr{E}_{\beta}$ in the definition of the derived inner sequence, we actually get an extender. The proof of the theorem comes from [Mi3].

Theorem 6.8. Let $\mathscr{M}=(M, \in, \delta, \mathscr{F}, \vec{\nu})$ be an expanded premouse which is normally $\left(\omega_{1}+1\right)$-iterable. Let $\left\langle E_{\alpha} \mid \alpha<\lambda\right\rangle$ be the inner sequence derived from $(\mathscr{F}, \vec{\nu})$. Then for all $\alpha<\lambda$ and $F, G \in \mathscr{F}_{\alpha}, F \uparrow \nu_{\alpha}$ agrees with $G \uparrow \nu_{\alpha}$ over $L\left[\left\langle E_{\alpha} \mid \alpha<\lambda\right\rangle\right]^{\mathscr{M}}$. 
Proof. Suppose $\mathscr{M}, \alpha, F, G$, etc., provide a counterexample. By taking a Skolem hull, we may assume $\mathscr{M}$ is countable. We compare $\mathscr{M}$ with itself. By Lemma 6.7 (with $\mathscr{M}=\mathscr{N}$ ) there are iteration trees $\mathscr{T}$ on $\mathscr{M}$ with last model $\mathscr{P}$ and $\mathscr{U}$ on $\mathscr{M}$ with last model $Q$ such that $\mathscr{P}$ is an initial segment of $Q$ or vice-versa. By symmetry, we may assume $\mathscr{P}$ is an initial segment of $Q$. Let $i: \mathscr{M} \rightarrow \mathscr{P}$ be the canonical embedding. So $i(F) \uparrow i\left(v_{\alpha}\right)$ disagrees with $i(G) \mid i\left(\nu_{\alpha}\right)$ over $L\left[i\left(\left\langle E_{\gamma} \mid \gamma<\lambda\right\rangle\right)\right]^{\mathscr{P}}$. Moreover, $L\left[i\left(\left\langle E_{\gamma} \mid \gamma<\lambda\right\rangle\right)\right]^{\mathscr{P}}$ is contained in $\mathscr{P} \cap Q$, as $\mathscr{P}$ is an initial segment of $Q$. Thus $i(F) \uparrow i\left(\nu_{\alpha}\right)$ disagrees with $i(G) \uparrow i\left(\nu_{\alpha}\right)$ over $\mathscr{P} \cap Q$. But $\mathscr{P}$ is an initial segment of $Q$, so there is an $H \in \mathscr{C}_{i(\alpha)}^{Q}$, and any such $H$ agrees with both $i(F) \uparrow i\left(\nu_{\alpha}\right)$ and $i(G) \uparrow\left(\nu_{\alpha}\right)$ over $\mathscr{P} \cap Q$. This is a contradiction.

We shall be dealing with models of the form $L\left(V_{\delta}\right)$ in what follows. Our metatheory includes the Axiom of Choice, so we may assert that every member of $V_{\delta}$ is wellorderable in $L\left(V_{\delta}\right)$. Clearly, $V_{\delta}$ may not itself be wellorderable in $L\left(V_{\delta}\right)$. Notice that if $L\left(V_{\delta}\right) \models$ " $\delta$ is Woodin", then $L\left(V_{\delta}\right) \models$ " $\delta$ is strongly inaccessible", and therefore $L\left(V_{\delta}\right)$ satisfies $<\delta$ dependent choices. This implies that one can force over $L\left(V_{\delta}\right)$ with a $<\delta$-closed partial order so as to adjoin a wellordering of $V_{\delta}$ without adding new elements of $V_{\delta}$. Solovay and Woodin have shown that if $L\left(V_{\delta}\right) \models$ " $\delta$ is Woodin" and if $G$ is $L\left(V_{\delta}\right)$-generic for this forcing, then $L\left(V_{\delta}\right)[G] \models \mathrm{ZFC}+$ " $\delta$ is Woodin". The reader who feels uncomfortable working in $L\left(V_{\delta}\right)$, without full choice, can avoid this in what follows by passing to $L\left(V_{\delta}\right)[G]$.

Definition 6.9. Let $(\mathscr{F}, \vec{\nu})$ be the maximal Doddage, and $\delta \in \mathrm{OR}$. Then the maximal $\delta$-Doddage is $\left\langle\left(\mathscr{F}_{\beta}, \nu_{\beta}\right) \mid \beta \in \operatorname{dom}(\mathscr{F}) \wedge \mathscr{F}_{\beta} \in V_{\delta}\right\rangle$.

Theorem 6.10. Suppose $L\left(V_{\delta}\right) \models$ “ $\delta$ is Woodin”, and $\forall \gamma<\delta\left(L\left(V_{\gamma}\right) \not \models\right.$ “ $\gamma$ is Woodin"). Let $\left\langle E_{\alpha} \mid \alpha<\delta\right\rangle$ be the inner sequence derived from the maximal $\delta$-Doddage. Then each $E_{\alpha}$ is an extender over $L\left[\left\langle E_{\alpha} \mid \alpha<\delta\right\rangle\right]$; moreover, $L\left[\left\langle E_{\alpha} \mid \alpha<\delta\right\rangle\right] \models$ " $\delta$ is Woodin".

Proof. Let $(\mathscr{F}, \vec{\nu})$ be the maximal $\delta$-Doddage. $(\mathscr{F}, \vec{\nu})$ is first-order definable over $V_{\delta}$ as the maximal Doddage; so $(\mathscr{F}, \vec{\nu}) \in L\left(V_{\delta}\right)$ and $L\left(V_{\delta}\right) \models$ " $(\mathscr{F}, \vec{\nu})$ is the maximal $\delta$-Doddage". As $\delta$ is an inaccessible limit of cardinals $\kappa$ which are $(\kappa+3)$-strong in $L\left(V_{\delta}\right), \operatorname{dom}(\mathscr{F})=\delta$.

Suppose $\eta<\delta$ and $F, G \in \mathscr{E}_{\eta}$ and $F$ and $G$ disagree over $L[\vec{E}]$. We shall derive a contradiction. Working in $L\left(V_{\delta}\right)$, where $\delta$ is strongly inaccessible, we can easily find a $\xi<\delta$ such that $\eta<\xi$ and an expanded premouse $\mathscr{P}=(P$, $\epsilon, \xi, \mathscr{F} \uparrow \xi, \vec{\nu} \uparrow \xi)$ such $V_{\xi}^{P}=V_{\xi}, F$ and $G$ disagree over $L[\langle E \gamma \mid \gamma<\xi\rangle]^{\mathscr{P}}$, and no $\gamma \leq \xi$ is Woodin in $L\left(V_{\gamma}\right)^{\mathscr{P}}$. Let $\mathscr{M} \preceq \mathscr{P}$ be countable. We would like to apply Theorem 6.8; unfortunately, we need that $\mathscr{M}$ is normally $\left(\omega_{1}+1\right)$ iterable for that, and from Theorem 4.3 and Corollary 2.4 we only get directly that $\mathscr{M}$ is normally $\omega_{1}$-iterable. But let $z$ be a real coding $\mathscr{M}$; we shall show $\mathscr{M}$ is $\omega_{1}^{V}$-iterable in $L[z]$. Since $\omega_{1}^{L[z]}<\omega_{1}^{V}$, we can then apply Theorem 6.8 
within $L[z]$ and reach the desired contradiction.

It will be enough to show that if $\mathscr{T} \in L[z]$ is a normal iteration tree on $\mathscr{M}$ of limit length $\lambda<\omega_{1}^{V}$, then there is a unique cofinal wellfounded branch of $\mathscr{T}$, and this branch is in $L[z]$. (Notice that Theorem 4.3 then implies that $\mathscr{T}$ cannot go bad at successor steps.) Uniqueness follows at once from Corollary 2.4. For existence, let $x$ be generic over $L[z]$ for the Levy collapse making $\lambda$ countable. Since $\omega_{1}^{V}$ is inaccessible in $L[z]$, there is such an $x$. By Theorem 4.3, there is a maximal wellfounded branch of $\mathscr{T}$, and by Corollary 2.4 any such branch is cofinal. It is a $\Sigma_{2}^{1}$ fact about any real $y \in L[z][x]$ coding $(\mathscr{M}, \mathscr{T})$ that $\mathscr{T}$ has a cofinal wellfounded branch, so $\mathscr{T}$ has such a branch $b$ in $L[z][x]$. Since $b$ is unique and the Levy collapse is homogeneous, $b \in L[z]$, as desired.

Thus if $\eta<\delta$ and $F, G \in \mathscr{E}_{\eta}$, then $F$ and $G$ agree over $L[\vec{E}]$. It follows that all $F \in \mathscr{E}_{\eta}$ agree with $E_{\eta}$ over $L[\vec{E}]$, so $E_{\eta}$ is an extender over $L[\vec{E}]$.

We work now in $L\left(V_{\delta}\right)$. As $\delta$ is inaccessible, if $A \subseteq \alpha<\delta$ and $A \in L[\vec{E}]$, then by a simple Löwenheim-Skolem argument we get a $\gamma<\delta$ such that $A \in$ $L\left[\left\langle E_{\beta} \mid \beta<\gamma\right\rangle\right]$. Thus there is a function $g: \delta \rightarrow \delta$ such that for all $\alpha<\delta$

$$
V_{\alpha}^{L[\vec{E}]} \subseteq L\left[\left\langle E_{\gamma} \mid \nu_{\gamma}<g(\alpha)\right\rangle\right] .
$$

Now fix $f: \delta \rightarrow \delta$ such that $f \in L[\vec{E}]$; we want to show that $\delta$ is Woodin with respect to $f$ in $L[\vec{E}]$. Let, for $\alpha<\delta$

$$
h(\alpha)=\text { least inaccessible } \theta \text { such that } f(\alpha)<\theta \text { and } g(f(\alpha))<\theta \text {. }
$$

Now Woodin cardinals are well known to be " $A$-strong" for arbitrary $A \subseteq$ $V_{\delta}$; taking $A$ to code $(\mathscr{E}, h, f)$, this means that there is a $\kappa<\delta$ and an elementary $j: V \rightarrow M$ with $\operatorname{crit}(j)=\kappa$ such that

(a) $h^{\prime \prime} \kappa \subseteq \kappa$ and $f^{\prime \prime} \kappa \subseteq \kappa$,

(b) $V_{j(h)(\kappa)} \in M$,

(c) $j(h)(\kappa)=h(\kappa)$ and $j(f)(\kappa)=f(\kappa)$,

(d) $j(\mathscr{E})$ | length $<h(\kappa)=\mathscr{E}$ | length $<h(\kappa)$.

(See Lemma 4.2 of [MS]. Incidentally, the proof that such a $j$ exists is a direct descendant of Mitchell's proof in [Mil] that large cardinal properties go from $V$ to his inner models.)

Let $F$ be the $(\kappa, h(\kappa))$ extender derived from $j$, and let $\rho<h(\kappa)$ be such that $f(\kappa)<\rho, g(f(\kappa))<\rho$, and $\rho=\operatorname{card}\left(V_{\rho}\right)$. Let $\gamma$ be least such that $\rho<\nu_{\gamma}$. Now at stage $\gamma$ in the construction of $(\mathscr{F}, \vec{\nu})$ we might have set $\nu_{\gamma}=\rho$ and put $F \mid \rho$ into $\mathscr{E}_{\gamma}$, except perhaps for the novelty condition. Since $\nu_{\gamma}>\rho$, the novelty condition failed, and we have an $\alpha<\gamma$ and $G \in \mathscr{E}_{\alpha}$ such that $\nu_{\alpha}=\rho$ and $G$ agrees with $F \mid \rho$ over $L\left[\left\langle E_{\beta} \mid \nu_{\beta} \leq \rho\right\rangle\right]$. But $g(\kappa)<\rho$, so all subsets of $\kappa$ in $L[\vec{E}]$ are in $L\left[\left\langle E_{\beta} \mid \nu_{\beta} \leq \rho\right\rangle\right.$, so $G$ agrees with $F\lceil\rho$ over $L[\vec{E}]$. Let $H=G \cap L[\vec{E}]=E_{\alpha} \cap L[\vec{E}]=F \mid \rho \cap L[\vec{E}]$. So $H$ is an 
extender in $L[\vec{E}]$, and we claim that, in $L[\vec{E}], H$ witnesses the Woodinness of $\delta$ with respect to $f$.

Consider the commutative diagram:

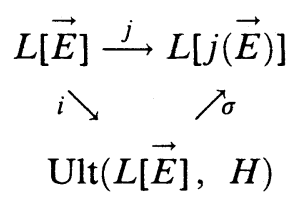

where $\sigma\left([a, \ell]_{H}^{L[\vec{E}]}\right)=j(\ell)(a)$ for all $a \in[\rho]^{<\omega}$ and functions $\ell \in L[\vec{E}]$. Now $\sigma \uparrow \rho=\mathrm{id}$, and as $\rho=\operatorname{card}\left(V_{\rho}\right), \sigma \uparrow V_{\rho}^{\mathrm{Ult}(L[\vec{E}], H)}=\mathrm{id}$. But $V_{f(\kappa)}^{L[\vec{E}]} \subseteq L[j(\vec{E})]$ as $L\left[\left\langle E_{\beta} \mid \nu_{\beta} \leq \rho\right\rangle\right] \subseteq L[j(\vec{E})]$ by property (d) of $j$. Thus

$$
V_{f(\kappa)}^{L[\vec{E}]} \subseteq \operatorname{Ult}(L[\vec{E}], H) .
$$

Further, $\sigma(i(f)(\kappa))=j(f)(\kappa)=f(\kappa)$. Since $f(\kappa)<\rho, i(f)(\kappa)=f(\kappa)$. This means that $H$ witnesses the Woodinness of $\delta$ with respect to $f$ in $L[\vec{E}]$.

We now have an inner model of the form $L[\vec{E}]$ satisfying that there is a Woodin cardinal. We show next that if $\vec{E}$ is, in a certain sense, minimal with this property, then in $L[\vec{E}]$ the natural wellorder of the reals (given by the order of construction) is $\Delta_{3}^{1}$.

Definition 6.11. Let $\mathscr{M}=(M, \in, \delta, \mathscr{F}, \vec{\nu})$ be an expanded premouse, and $\left\langle E_{\alpha}\right| \alpha\langle\eta\rangle$ its derived inner sequence. We say $\mathscr{M}$ is minimal iff

(1) $\mathscr{M} \models$ “ $(\mathscr{F}, \vec{\nu})$ is the maximal $\delta$-Doddage",

(2) $\forall \alpha<\eta\left(E_{\alpha}\right.$ is an extender over $\left.L\left[\left\langle E_{\beta} \mid \beta<\eta\right\rangle\right]^{\mathscr{K}}\right)$,

(3) $\forall \gamma<\delta\left(L\left[\left\langle E_{\alpha} \mid \alpha<\min (\gamma, \eta)\right\rangle\right]^{\mathscr{}} \models\right.$ " $\gamma$ is not Woodin"),

(4) $\forall \gamma<\delta\left(L\left(V_{\gamma}\right)^{\mathscr{M}} \models\right.$ “ $\gamma$ is not Woodin"),

and

(5) if $L\left(V_{\delta}\right)^{\mathscr{K}} \models$ " $\delta$ is Woodin", then $\eta=\delta$ and $L\left[\left\langle E_{\alpha} \mid \alpha<\delta\right\rangle\right]^{\mathscr{K}} \models$ " $\delta$ is Woodin".

The proof of Theorem 6.10 shows that if $\mathscr{M} \models$ " $\delta$ is inaccessible in $L\left(V_{\delta}\right)$ and a limit of cardinals $\kappa$ which are $(\kappa+3)$-strong" and if $\mathscr{M}$ satisfies (1) and (3), then $\mathscr{M}$ satisfies (2), (4), and (5). This justifies the use of the term "minimal".

Life would be simpler if the property of being (a real coding) a countable minimal $\omega_{1}$-iterable expanded premouse were a $\Pi_{2}^{1}$ property. However, if there is a $\delta$ such that $\delta$ is Woodin in $L\left(V_{\delta}\right)$ and $V_{\delta}^{\#}$ exists, then this is not the case. [Although we do not need it later, we sketch the proof of this fact. Let $P$ be the property in question. Let $\vec{E}$ be the inner sequence derived from the maximal $\delta$-Doddage. Applying Theorem 6.10 within $L\left(V_{\delta}\right)$, we have a $\gamma \leq \delta$ such that $L[\vec{E} \mid \gamma] \models$ " $\gamma$ is Woodin". Let $\gamma \leq \operatorname{lh}(\vec{E})$ be least such that 


$$
\begin{array}{r}
L[\vec{E} \uparrow \gamma] \models \text { “ } \gamma \text { is a Woodin cardinal". Then for all } x \subseteq \omega \\
x \in L\left[\vec{E}\lceil\gamma] \text { iff } \exists \mathscr{M} \text { ( } \mathscr{M} \text { is a countable, minimal, normally } \omega_{1}\right. \text {-iterable } \\
\text { expanded premouse, and } x \in L[\vec{G}]^{\mathscr{M}} \text {, where } \\
\vec{G} \text { is the derived inner sequence of } \mathscr{M} \text { ). }
\end{array}
$$

The $\Rightarrow$ direction is a simple Löwenheim-Skolem argument. The $\Leftarrow$ direction comes from comparing $L[\vec{G}]^{\mathscr{N}}$ with $L[\vec{E} \mid \gamma]$. We have enough iterability to do the comparison because $\vec{E} \uparrow \gamma, \mathscr{M} \in L\left(V_{\delta}\right)$, and $V_{\delta}^{\#}$ exists. (See $\S 5$.) Therefore, if $P$ is $\Pi_{2}^{1}$, then $P(\omega) \cap L[\vec{E} \uparrow \gamma]$ is $\Sigma_{3}^{1}$. On the other hand, we shall show later in this section that $P(\omega) \cap L[\vec{E} \uparrow \gamma] \subseteq Q_{3}$, and Woodin (unpublished) has recently shown $Q_{3} \subseteq P(\omega) \cap L[\vec{E} \uparrow \gamma]$. (See [KMSo] for the definition and basic theory of $Q_{3}$.) Since $Q_{3}$ is not $\Sigma_{3}^{1}, P$ is not $\Pi_{2}^{1}$.]

In order to get a $\Delta_{3}^{1}$ wellorder, we need a $\Pi_{2}^{1}$ approximation to the notion of $\omega_{1}$-iterability for countable, minimal expanded premice. Definition 6.12 records such an approximation. Recall that if $b$ is a branch of an iteration tree $\mathscr{T}$ on $\mathscr{M}$, then $\mathscr{M}_{b}=\lim _{\alpha \in b} \mathscr{M}_{\alpha}$ need not be wellfounded. Let us say that $\mathscr{M}_{b}$ is $\gamma$-wellfounded, for $\gamma \in \mathrm{OR}$, iff $\mathscr{M}_{b}$ is wellfounded or the wellfounded part of $\mathscr{M}_{b}$ has ordinal rank $\geq \gamma$.

Definition 6.12. A $\Pi_{2}^{1}$ mouse is a countable expanded premouse $\mathscr{M}=(M$, $\in, \delta, \mathscr{F}, \vec{\nu})$ such that $\mathscr{M} \models$ “ $(\mathscr{F}, \vec{\nu})$ is the maximal $\delta$-Doddage", and such that for every countable normal iteration tree $\mathscr{T}$ on $\mathscr{M}$ :

(1) if $\operatorname{lh}(\mathscr{T})$ is a limit ordinal, then for each $\gamma<\omega_{1}$ there is a maximal branch $b$ of $\mathscr{T}$ such that $\mathscr{M}_{b}$ is $\gamma$-wellfounded, and

(2) if $\operatorname{lh}(\mathscr{T})=\alpha+1, \mathscr{M}_{\alpha} \models$ “ $E$ is an extender", and $\operatorname{crit}(E)<\rho^{\mathscr{T}}(\beta, \alpha)$ where $\beta \leq \alpha$, then either $\operatorname{Ult}\left(\mathscr{M}_{\beta}^{\mathscr{T}}, E\right)$ is wellfounded or $\forall \gamma<\omega_{1} \exists b$ ( $b$ is a maximal branch of $\mathscr{T}$ and $\mathscr{M}_{b}$ is $\gamma$-wellfounded).

Notice that $\gamma$-wellfoundedness is uniformly $\Sigma_{1}^{1}$ in any code for $\gamma$. It follows that $\left\{x \in{ }^{\omega} \omega \mid x\right.$ codes a $\Pi_{2}^{1}$ mouse $\}$ is $\Pi_{2}^{1}$. Were we to make the more natural requirement in, for example, clause (1) of Definition 6.12 that there be a maximal fully wellfounded branch of $\mathscr{T}$, then we would have a $\Pi_{3}^{1}$ rather than a $\Pi_{2}^{1}$ notion of mousehood. Our uniqueness theorem, Corollary 2.4 , implies that for minimal premice this stronger $\Pi_{3}^{1}$ condition is equivalent to $\omega_{1}$-iterability with respect to normal trees. In particular, any normally $\omega_{1}$-iterable minimal premouse is a $\Pi_{2}^{1}$ mouse.

It is not true that any two countable, minimal $\Pi_{2}^{1}$ mice can be compared with one another. However, we do get the following comparison lemma:

Lemma 6.13. Let $\mathscr{M}$ be a countable, minimal, $\omega_{1}$-iterable expanded premouse. Let $\mathscr{N}$ be a minimal $\Pi_{2}^{1}$ mouse. Suppose $(\mathscr{M}, \mathscr{N})^{\#}$ exists. Then there are countable iteration trees $\mathscr{T}$ on $\mathscr{M}$ and $\mathscr{U}$ on $\mathscr{N}$ such that either 
(a) $\mathscr{T}$ and $\mathscr{U}$ have last models of $\mathscr{P}$ and $\mathscr{Q}$ respectively, and either $\mathscr{P}$ is an initial segment of $Q$ or vice-versa, or

(b) $\mathscr{T}$ and $\mathscr{U}$ have limit length, and there are cofinal branches $b$ of $\mathscr{T}$ and c of $\mathscr{U}$ such that $\mathscr{M}_{b}$ is wellfounded, and $\mathscr{M}_{b}$ is an initial segment of $\mathscr{N}_{c}$.

Remark. We need alternatives (b) in Lemma 6.13 to accommodate the possbility of ending the comparison process by choosing an illfounded iterate $\mathscr{N}_{c}$ of $\mathscr{N}$.

Proof. The proof is very close to that of Lemma 6.7. We define iteration trees $\mathscr{T}_{\xi}$ and $\mathscr{U}_{\xi}$ on $\mathscr{M}$ and $\mathscr{N}$ respectively, by induction on $\xi$, maintaining the induction hypotheses (a), (c)-(e) of that proof. Instead of induction hypothesis (b) of Lemma 6.7 (which stated that $\mathscr{T}_{\xi}$ and $\mathscr{U}_{\xi}$ were plays according to certain iteration strategies) we maintain inductively

(b) ' If $\mathscr{S}=\bigcup_{\xi<\lambda} \mathscr{T}_{\xi}$ is an iteration tree of limit length $\eta$ and $\mathscr{T}_{\lambda}$ is defined, then $\operatorname{lh}\left(\mathscr{T}_{\lambda}\right)=\eta+1$ and $\left\{\alpha \mid \alpha\left(\mathrm{T}_{\lambda}\right) \eta\right\}$ is the unique cofinal wellfounded branch of $\mathscr{S}$. Similarly, if $\mathscr{S}=\bigcup_{\xi<\lambda} \mathscr{U}_{\xi}$ is an iteration tree of limit length $\eta$ and $\mathscr{U}_{\lambda}$ is defined, then $\ln \left(\mathscr{U}_{\lambda}\right)=\eta+1$ and $\left\{\alpha \mid \alpha\left(\mathrm{U}_{\lambda}\right) \eta\right\}$ is the unique cofinal wellfounded branch of $\mathscr{S}$.

Of course, (b)' implies that the $\mathscr{T}_{\xi}$ 's are plays according to any and all iteration strategies for $\mathscr{M}$.

If we ever reach a limit stage $\lambda<\omega_{1}$ so that it is impossible to define $\mathscr{T}_{\lambda}$ and $\mathscr{U}_{\lambda}$ so as to satisfy $(\mathrm{b})^{\prime}$, then we stop the construction at $\lambda$. And, as in Lemma 6.7, we stop the construction at any stage $\xi$ such that the last models of $\mathscr{T}_{\xi}$ and $\mathscr{U}_{\xi}$ are "lined up"; that is, one is an initial segment of the other.

It is also ostensibly possible that at some stage $\xi$, the ultrapower to be taken in extending $\mathscr{U}_{\xi}$ to $\mathscr{U}_{\xi+1}$ is illfounded. In this case too we stop the construction at $\xi$; we shall argue later that in fact the construction never stops for this reason. Notice that because the construction of $\mathscr{T}$ follows any and all iteration strategies for $\mathscr{M}$, we never encounter illfounded ultrapowers at successor steps in the construction of $\mathscr{T}$.

Claim. The construction stops at some $\alpha<\omega_{1}$.

Proof. Assume otherwise. Notice then that the construction is absolute to $L[(\mathscr{M}, \mathscr{N})]$, the smallest transitive proper class model of ZF to which $(\mathscr{M}, \mathscr{N})$ belongs. This is clear once we show that if $\lambda<\omega$ is a limit ordinal and $\mathscr{R}=$ $\bigcup_{\alpha<\lambda} \mathscr{T}_{\alpha}$ has limit length, then the unique cofinal wellfounded branch $b$ of $\mathscr{R}$ is in $L[(\mathscr{M}, \mathscr{N})]$. (One also needs the parallel fact for $\bigcup_{\alpha<\lambda} \mathscr{U}_{\alpha}$, which is proved in the same way.) We may assume by induction that $\mathscr{R} \in L[(\mathscr{M}, \mathscr{N})]$. Let $\eta=\max (\operatorname{card}(\mathscr{R}), \operatorname{card}(\mathscr{M}))^{L[(\mathscr{N})]}$, and let $x$ be generic over $L[(\mathscr{M}, \mathscr{N})]$ for the Levy poset making $\eta$ countable. As $\eta<\omega_{1}$ and $(\mathscr{M}, \mathscr{N})^{\#}$ exists, there is such an $x$ in $V$. It is a $\Sigma_{2}^{1}$ fact about any real coding $\mathscr{R}$ that $\mathscr{R}$ has a cofinal wellfounded branch. Thus by Shoenfield absoluteness, $\mathscr{R}$ has a cofinal wellfounded branch in $L[(\mathscr{M}, \mathscr{N})][x]$. As $b$ is the unique such branch in $V, b$ is the unique such branch in $L[(\mathscr{M}, \mathscr{N})][x]$. The homogeneity of the collapsing poset implies $b \in L[(\mathscr{M}, \mathscr{N})]$.

Let $\eta=\max \left(\operatorname{card}(\mathscr{M})^{+}, \operatorname{card}(\mathscr{N})^{+}\right)^{L[(\mathscr{M}, \mathcal{N})]}$. So $\eta<\omega_{1}$, and $\mathscr{T}_{\eta}$ and $\mathscr{U}_{\eta}$ 
are defined and in $L[(\mathscr{M}, \mathcal{N})]$. Working in $L[(\mathscr{M}, \mathscr{N})]$, we can now reach the same contradiction we reached in the proof of Lemma 6.7. This proves the claim.

Suppose the construction stops at $\lambda<\omega_{1}$. If $\mathscr{T}_{\lambda}$ and $\mathscr{U}_{\lambda}$ exist and their last models are lined up, then we are done. The second possibility is that $\lambda$ is a limit ordinal, and one of $\mathscr{R}=\bigcup_{\alpha<\lambda} \mathscr{T}_{\alpha}$ and $\mathscr{S}=\bigcup_{\alpha<\lambda} \mathscr{U}_{\alpha}$ has limit length but does not have a unique cofinal wellfounded branch. Suppose this happens, and suppose first that $\mathscr{S}$ has limit length but does not have a cofinal wellfounded branch.

Since $\mathscr{N}$ is a $\Pi_{2}^{1}$ mouse, for each $\gamma<\omega_{1}$ there is a maximal, $\gamma$-wellfounded branch $b_{\gamma}$ of $\mathscr{S}$. Fix $\eta \leq \operatorname{lh}(\mathscr{S})$ such that $\sup \left(b_{\gamma}\right)=\eta$ for cofinally many $\gamma<$ $\omega_{1}$. We may assume $\sup \left(b_{\gamma}\right)=\eta$ for all $\gamma<\omega_{1}$. Now no $b_{\gamma}$ is wellfounded; if $\eta=\ln (\mathscr{S})$ this is our hypothesis, and if $\eta<\operatorname{lh}(\mathscr{S})$ this is so because the construction did not stop before $\lambda$. Thus the wellfounded part of $\mathscr{N}_{b_{\gamma}}$ has order type $\geq \gamma$.

Let $\sigma=\lambda$ if $\eta=\ln (\mathscr{S})$, and let $\sigma<\lambda$ be least such that $\mathscr{U}_{\sigma}$ has length $\geq \eta$ otherwise. (So, in fact, $\ln \left(\mathscr{U}_{\sigma}\right)=\eta+1$.) Recall that $\rho_{\alpha}$ is the "length" of a least disagreement between the last models of $\mathscr{T}_{\alpha}$ and $\mathscr{U}_{\alpha}$, for all $\alpha<\lambda$. Let $\theta=\sup \left(\left\{\rho_{\alpha} \mid \alpha<\sigma\right\}\right)$.

Notice that if $b$ and $c$ are cofinal branches of $\mathscr{S} \mid \eta$ having $\theta$ in their wellfounded parts, then $V_{\theta}^{\mathscr{N}_{b}}=V_{\theta}^{\mathscr{N}_{c}}$.

Let $X$ be the common value of $V_{\theta}^{\mathscr{N}_{b_{\gamma}}}$ for all $\gamma>\theta$; then by Theorem 2.2. $L_{\omega_{1}}(X) \vDash " \theta$ is Woodin $\wedge X=V_{\theta}$ ". Notice also that $X^{\#}$ exists because $X \in L[\mathscr{M}, \mathscr{N}]$, so $L_{\omega_{1}}(X) \vDash \mathrm{ZF}$. Every member of $X$ has a wellordering in $X$ because $X=V_{\theta}^{\mathscr{P}}$ for some model $\mathscr{P}$ of AC. We can therefore apply the proof of Theorem 6.10 to $L_{\omega_{1}}(X)$ (in the role of $L\left(V_{\delta}\right)$ in that theorem) to see that if $\left\langle F_{\alpha} \mid \alpha<\theta\right\rangle$ is the inner sequence derived from the maximal $\theta$-Doddage of the model $L_{\omega_{1}}(X)$, then

$$
L_{\omega_{1}}\left[\left\langle F_{\alpha} \mid \alpha<\theta\right\rangle\right] \models \text { “ } \theta \text { is Woodin". }
$$

If $\eta<\operatorname{lh}(\mathscr{S})$, then $V_{\theta}^{\mathscr{N}_{\eta}}=X$, so $\left\langle F_{\alpha} \mid \alpha<\theta\right\rangle$ is the inner sequence derived from the maximal $\theta$-Doddage of $\mathscr{N}_{\eta}$. Since $\mathscr{N}_{\eta}$ is minimal, $\theta=\delta^{\mathscr{N}}$.

Since the derived inner sequences of $\mathscr{N}_{\eta}$ and the last model of $\mathscr{T}_{\sigma}$ agree below $\theta=\sup \left\{\rho_{\alpha} \mid \alpha<\sigma\right\}, \mathscr{N}_{\eta}$ is an initial segment of the last model of $\mathscr{T}_{\sigma}$, or vice-versa. Thus the construction stopped at $\eta<\operatorname{lh}(\mathscr{S})$, a contradiction.

Thus $\eta=\operatorname{lh}(\mathscr{S})$. Let $b$ be a cofinal, wellfounded branch of $\mathscr{T}_{\lambda}$.

Let $\left\langle E_{\alpha} \mid \alpha<\theta\right\rangle$ be the length $\theta$ initial segment of the inner sequence derived from the Doddage of $\mathscr{M}_{b}$. Notice $E_{\alpha}$ agrees with $F_{\alpha}$ over $\mathscr{M}_{b} \cap L_{\omega_{1}}(X)$ for all $\alpha<\theta$. It follows that

$$
L\left[\left\langle E_{\alpha} \mid \alpha<\theta\right\rangle\right]^{\mathscr{M}_{b}} \models \text { “ } \theta \text { is Woodin". }
$$

As $\mathscr{M}_{b}$ is minimal, this implies that $\mathscr{M}_{b}$ is an initial segment of $\mathscr{N}_{b_{y}}$, for all $\gamma>\mathrm{OR} \cap \mathscr{M}_{b}$ so that conclusion (b) of Lemma 6.13 holds, as desired. 
Thus we may assume $\mathscr{S}=\bigcup_{\alpha<\lambda} \mathscr{U}_{\alpha}$ has a cofinal wellfounded branch $c$. As $\mathscr{M}$ is $\omega_{1}$-iterable, $\mathscr{R}=\bigcup_{\alpha<\lambda} \mathscr{T}_{\alpha}$ has a cofinal wellfounded branch $b$. Since the construction stopped at $\lambda$, one of $\mathscr{S}$ and $\mathscr{R}$ has a second cofinal wellfounded branch $d$, and by symmetry we may as well assume $d$ is a branch of $\mathscr{R}$. Let $\theta=\sup \left\{\rho_{\alpha} \mid \alpha<\lambda\right\}$ and $\nu=\inf \left(\mathrm{OR} \cap \mathscr{M}_{b}, \mathrm{OR} \cap \mathscr{M}_{d}\right)$. Say $\nu=\mathrm{OR} \cap \mathscr{M}_{b}$. By Theorem 2.2,

$$
L\left(V_{\theta}\right)^{\mathscr{M}_{b}} \models \text { “ } \theta \text { is Woodin", }
$$

and arguing as above this implies that $\mathscr{M}_{b}$ is an initial segment of $\mathscr{N}_{c}$ or viceversa. Let $\mathscr{T}$ be the iteration tree of length $=\operatorname{lh}(\mathscr{R})+1$ extending $\mathscr{R}$ and having last model $\mathscr{M}_{b}$. Let $\mathscr{U}$ extend $\mathscr{S}$ and have last model $\mathscr{N}_{c}$. Then $\mathscr{T}$ and $\mathscr{U}$ witness conclusion (a) of Lemma 6.13.

Finally, we must show that the construction cannot stop at a successor step because we are asked to form an illfounded ultrapower in $\mathscr{U}$. Let $\mathscr{S}$ be the iteration tree on $\mathscr{N}$ which we are asked to extend by forming an illfounded ultrapower. By part (2) of Definition 6.12, for each $\gamma<\omega_{1}$ there is a maximal wellfounded branch $b_{\gamma}$ of $\mathscr{S}$. We can fix $\eta \leq \operatorname{lh}(\mathscr{S})$ such that $\eta=\sup \left(b_{\gamma}\right)$ for cofinally many $\gamma<\omega_{1}$. Since $\mathscr{S}$ has successor length, $\eta<\operatorname{lh}(\mathscr{S})$. We can now use the arguments given earlier to show that the construction must have stopped before we reached $\mathscr{S}$, a contradiction.

Let $\delta$ be least such that $\delta$ is Woodin in $L\left(V_{\delta}\right)$, and let $\vec{E}$ be the inner sequence derived from the maximal $\delta$-Doddage. Applying Theorem 6.10 within $L\left(V_{\delta}\right)$, we see that $\ln (\vec{E})=\delta$ and $L[\vec{E}] \models$ " $\delta$ is Woodin".

Definition 6.14. Let $\vec{E}$ be as described above. Then $\vec{E}^{\text {min }}=\vec{E} \uparrow \gamma$, where $\gamma \leq \operatorname{lh}(\vec{E})$ is least such that $L[\vec{E} \uparrow \gamma] \models$ " $\gamma$ is Woodin".

Our comparison lemma, 6.13, implies that every real in $L\left[\vec{E}^{\mathrm{min}}\right]$ is definable. More precisely, let us say that a real $x \in{ }^{\omega} \omega$ is $\Delta_{3}^{1}$ in an ordinal $\alpha<\omega_{1}$ iff there is a $\Sigma_{3}^{1}$ relation $R \subseteq{ }^{\omega} \omega \times{ }^{\omega} \omega$ such that whenever $y \in{ }^{\omega} \omega$ is a code (in some standard coding system) for $\alpha$, then $x$ is the unique $z$ such that $R(z, y)$. We let $Q_{3}=\left\{x \mid \exists \alpha<\omega_{1}\left(x\right.\right.$ is $\Delta_{3}^{1}$ in $\left.\left.\alpha\right)\right\}$. The set $Q_{3}$ has many other natural characterizations; we refer the reader to [KMSo] for further information. We shall show that every real in $L\left[\vec{E}^{\mathrm{min}}\right]$ is in $Q_{3}$.

Lemma 6.15. Assume $\forall x \in{ }^{\omega} \omega\left(x^{\#}\right.$ exists). Suppose $\mathscr{M}$ is a normally $\omega_{1}$ iterable expanded premouse and $\mathscr{M}$ is minimal. Let $x \in{ }^{\omega} \omega \cap L[\vec{E}]^{\mathscr{M}}$, where $\vec{E}$ is the derived inner sequence of $\mathscr{M}$; then $x \in Q_{3}$.

Proof. Since any premouse embedded in a normally $\omega_{1}$-iterable premouse is itself normally $\omega_{1}$-iterable, we may assume $\mathscr{M}$ is countable. Let $\alpha<\omega_{1}$ be such that $x$ is the $\alpha$ th real, in order of construction, in $L[\vec{E}]^{\mathscr{M}}$. We claim that for all reals $z$,

$$
\begin{array}{r}
z=x \text { iff } \exists \mathscr{N}\left(\mathscr{N} \text { is a countable, minimal, } \Pi_{2}^{1}\right. \text { mouse with derived } \\
\text { inner sequence } \left.\vec{F} \text {, and } z \text { is the } \alpha \text { th real in } L[\vec{F}]^{\mathcal{N}}\right) .
\end{array}
$$


The left to right direction is trivial; take $\mathscr{N}=\mathscr{M}$. Let $\mathscr{N}, \vec{F}$, and $z$ be as on the right. Since $\mathscr{M}$ and $\mathscr{N}$ are countable, $(\mathscr{M}, \mathscr{N})^{\#}$ exists. Our comparison lemma, 6.13, yields premice $\mathscr{P}$ and $Q$ and embeddings $i: \mathscr{M} \rightarrow \mathscr{P}$ and $j: \mathcal{N} \rightarrow Q$, with $\mathscr{P}$ an initial segment of $Q$ or vice-versa. (We take the liberty of allowing $Q$ to be illfounded here, in which case $\mathscr{P}$ must be an initial segment of $Q$.) We also have that $i$ is the identity on $\left({ }^{\omega} \omega \cup\left\{{ }^{\omega} \omega\right\}\right)^{\mathscr{M}}$ and $j$ is the identity on $\left({ }^{\omega} \omega \cup\left\{{ }^{\omega} \omega\right\}\right)^{\mathscr{N}}$; moreover, $i$ and $j$ preserve the order of construction. Thus $i(x)=x$ is the $\alpha$ th real of $L[i(\vec{E})]^{\mathscr{P}}$ and $j(z)=z$ is the $\alpha$ th real of $L[j(\vec{F})]^{Q}$. It follows that $z=x$.

Since being a $\Pi_{2}^{1}$ mouse is a $\Pi_{2}^{1}$ property, the equivalence displayed above shows $x$ is $\Delta_{3}^{1}$ in $\alpha$.

Lemma 6.15 yields at once

Theorem 6.16. If $\exists \delta\left(L\left(V_{\delta}\right) \models " \delta\right.$ is Woodin"), then $\left({ }^{\omega} \omega \cap L\left[\vec{E}^{\min }\right]\right) \subseteq Q_{3}$.

[KMSo] shows that every $\Sigma_{2}^{1}$ correct model of $\Delta_{2}^{1}$ determinacy has $Q_{3}$ properly included in its set of reals. So if $\exists \delta\left(L\left(V_{\delta}\right) \models\right.$ “ $\delta$ is Woodin"), then $L\left[\vec{E}^{\mathrm{min}}\right]$ satisfies “ $\exists \delta(\delta$ is Woodin $)$ and $\neg \Delta_{2}^{1}$ determinacy". In fact, the calculations behind Theorem 6.16 nearly show that $L\left[\vec{E}^{\mathrm{min}}\right] \models{ }^{\prime} \mathrm{CH}$ and ${ }^{\omega} \omega$ has a $\Delta_{3}^{1}$ wellorder." Unfortunately, they fall short of this because the premice and iteration trees over which one must quantify in defining the order of construction on ${ }^{\omega} \omega \cap L\left[\vec{E}^{\mathrm{min}}\right]$ need not themselves lie in $L\left[\vec{E}^{\mathrm{min}}\right]$. This difficulty affects all attempts to answer questions concerning the first-order theory of $L\left[\vec{E}^{\mathrm{min}}\right]$, and its full solution involves fine structure theory (see [MiS]). For the questions at hand, however, there are expedients available.

The first is due to Woodin. Assume $\Delta_{2}^{1}$ determinacy. Suppose $\delta$ is Woodin in $L\left(V_{\delta}\right)$, let $\vec{E}$ be the inner sequence derived from the maximal $\delta$-Doddage, and let $\gamma \leq \delta$ be least such that $L[\vec{E} \uparrow \gamma]\left(Q_{3}\right) \models$ " $\gamma$ is Woodin". The comparison process adapts to models with a certain fixed set of reals thrown in at the first stage of the construction; we can work above any set which will not be moved by the embeddings. So the proof of Theorem 6.10 shows such a $\gamma$ exists. Let $x \in{ }^{\omega} \omega$ code an enumeration of $Q_{3}$ and $y \in{ }^{\omega} \omega \cap L[\vec{E} \uparrow \gamma]\left(Q_{3}\right)$; then $y \in L[\vec{E} \mid \gamma][x]$, so the proof of Theorem 6.16 shows that $y \in Q_{3}(x)$. But [KMSo] shows that if $y \in Q_{3}(x)$ for every $x \in{ }^{\omega} \omega$ enumerating $Q_{3}$, then $y \in Q_{3}$. Thus ${ }^{\omega} \omega \cap L\left[\vec{E}\lceil\gamma]\left(Q_{3}\right)=Q_{3}\right.$. But [KMSo] shows that in $L\left(Q_{3}\right) \mathrm{CH}$ holds and ${ }^{\omega} \omega$ has a $\Delta_{3}^{1}$ wellorder. It follows that in $L[\vec{E} \backslash \gamma]\left(Q_{3}\right) \mathrm{CH}$ holds, ${ }^{\omega} \omega$ has a $\Delta_{3}^{1}$ wellorder, and $\gamma$ is Woodin.

As we remarked earlier, Woodin has recently shown that if $\exists \delta(\delta$ is Woodin in $L\left(V_{\delta}\right)$ and $V_{\delta}^{\#}$ exists), then ${ }^{\omega} \omega \cap L\left[\vec{E}^{\mathrm{min}}\right]=Q_{3}$. The proof involves a good deal more than the argument just given. The key is the remarkable fact, due to 
Woodin, that under these hypotheses there is a partial order $\mathbb{P} \in L\left[\vec{E}^{\mathrm{min}}\right]$ such that every $x \in{ }^{\omega} \omega$ is $\mathbb{P}$-generic over $L\left[\vec{E}^{\text {min }}\right]$.

There is a second way to get a model in which there is a Woodin cardinal, $\mathrm{CH}$ holds, and ${ }^{\omega} \omega$ has a $\Delta_{3}^{1}$ wellorder. It is due to Jensen [J2], and it involves no descriptive set theory. We describe it now.

Lemma 6.17. Let $\delta$ be Woodin; then there is $a \gamma \leq \delta$ and an expanded premouse $\mathscr{M}=(M, \in, \gamma, \mathscr{F}, \vec{\nu})$ such that

(a) $M$ is a proper class,

(b) $\mathscr{M}$ is minimal,

(c) $\mathscr{M} \models$ " $\gamma$ is Woodin",

(d) if $\left\langle E_{\alpha} \mid \alpha<\gamma\right\rangle$ is the inner sequence derived from $(\mathscr{F}, \vec{\nu})$, then ${ }^{\omega} \omega \cap M \subseteq L\left[\left\langle E_{\alpha} \mid \alpha<\gamma\right\rangle\right]$.

Proof. We define by induction on $i \in \omega$ expanded premice $\mathscr{M}_{i}=\left(M_{i}, \in\right.$, $\left.\gamma_{i}, \overrightarrow{\mathscr{F}}_{i}, \vec{\nu}_{i}\right)$ satisfying (a) and (c). Set

$$
M_{0}=V, \gamma_{0}=\delta,\left(\mathscr{F}_{0}, \vec{\nu}_{0}\right)=\text { the maximal } \gamma \text { Doddage. }
$$
Set

Now suppose $\mathscr{M}_{i}$ is given, and let $\vec{E}_{i}$ be the derived inner sequence of $\mathscr{M}_{i}$.

$$
\begin{aligned}
\gamma_{i+1} & =\text { least } \eta \leq \gamma_{i} \text { such that } L\left[\vec{E}_{i} \uparrow \eta\right] \models \text { " } \eta \text { is Woodin", } \\
M_{i+1} & =L\left[\vec{E}_{i} \uparrow \gamma_{i+1}\right], \\
\left(\mathscr{F}_{i+1}, \vec{\nu}_{i+1}\right) & =\text { the maximal } \gamma_{i+1} \text {-Doddage of } M_{i+1} .
\end{aligned}
$$

Notice that $\gamma_{i+1}$ exists by Theorem 6.10.

Fix $i_{0}$ such that $\gamma_{i}=\gamma_{i_{0}}$ for all $i \geq i_{0}$. Notice that $\mathscr{M}_{i}$ is minimal for all $i \geq i_{0}$. It is enough then to find $i \geq i_{0}$ such that ${ }^{\omega} \omega \cap M_{i} \subseteq M_{i+1}$, for then $\mathscr{M}_{i}$ has the properties required in Lemma 6.17.

Fix for a while an arbitrary $i \geq i_{0}$, and suppose ${ }^{\omega} \omega \cap M_{i} \nsubseteq M_{i+1}$. Pick $x \in{ }^{\omega} \omega \cap\left(M_{i} \backslash M_{i+1}\right)$. We work now inside the universe $M_{i-1}$. Since $x \in M_{i}$, we can by Corollary 5.11 find in $M_{i-1}$ a countable, minimal, normally $\omega_{1}$ iterable expanded premouse $\mathscr{N}$ such that $x \in L[\vec{E}]^{\mathscr{N}}$, where $\vec{E}$ is the derived inner sequence of $\mathscr{N}$. We claim that ${ }^{\omega} \omega \cap M_{i+1} \subseteq \mathscr{N}$. For otherwise, let $y \in{ }^{\omega} \omega \cap\left(M_{i+1} \backslash \mathscr{N}\right)$. Working inside $M_{i}$, we can by Corollary 5.11 find a countable, minimal, normally $\omega_{1}$-iterable expanded premouse $\mathscr{M}$ such that $y \in L[\vec{E}]^{\mathscr{M}}$, where $\vec{E}$ is the derived inner sequence of $\mathscr{M}$. Now $\mathscr{M}$ is a $\Pi_{2}^{1}$ mouse in $M_{i}$, and so by Shoenfield absoluteness, $\mathscr{M}$ is a $\Pi_{2}^{1}$ mouse in $M_{i-1}$ (although $\mathscr{M}$ may no longer be $\omega_{1}$-iterable in $M_{i-1}$ ). We can therefore apply the comparison Lemma 6.13 to $\mathscr{M}$ and $\mathscr{N}$ inside $M_{i-1}$. Since $y \notin L[\vec{E}]^{\mathscr{N}}$ and $x \notin L[\vec{E}]^{\mathscr{K}}$, neither can iterate to an initial segment of the other, a contradiction. Thus ${ }^{\omega} \omega \cap M_{i+1} \subseteq \mathscr{N}$. As $\mathscr{N}$ is countable in $M_{i-1}$, we see also that $\omega_{1}^{M_{i+1}}<\omega_{1}^{M_{i-1}}$. 
If, then, ${ }^{\omega} \omega \cap M_{i} \nsubseteq M_{i+1}$ for all $i \geq i_{0}$, then for all $i \geq i_{0}, \omega_{1}^{M_{i+1}}<\omega_{1}^{M_{i-1}}$. Since $\omega_{1}^{M_{i+1}} \leq \omega_{1}^{M_{i}}$ for all $i$, this is a contradiction.

The lemma yields at once the $n=1$ case of the main theorem.

Theorem 6.18. Let $\mathscr{M}$ have the properties described in Lemma 6.17, and let $\vec{E}$ be the derived inner sequence of $\mathscr{M}$. Then $L[\vec{E}] \models \mathrm{CH}+$ “ $\mathbb{R}$ has a $\Delta_{3}^{1}$ wellorder".

Proof. The $\Delta_{3}^{1}$ wellorder is just the order $<_{c}$ of construction in $L[\vec{E}]$. We claim that for $x, y \in \mathbb{R} \cap L[\vec{E}]$ the following is true in $L[\vec{E}]$ :

$$
\begin{array}{r}
x<_{c} y \text { iff } \exists \mathscr{N}(\mathscr{N} \text { is a countable, minimal, } \\
\left.\Pi_{2}^{1} \text { mouse, and } \mathscr{N} \models x<_{c} y\right) .
\end{array}
$$

Clearly, the right-hand side is $\Sigma_{3}^{1}$, so the equivalence shows $<_{c}$ is $\Delta_{3}^{1}$ in $L[\vec{E}]$. If $x<_{c} y$, then in $\mathscr{M}$ we can find a countable $\mathscr{N} \preccurlyeq\left(V_{\alpha}^{\mathscr{M}}, \in, \delta^{\mathscr{M}}, \mathscr{F}^{\mathscr{M}}\right.$, $\bar{\nu}^{\mathscr{N}}$ ), for some $\alpha$, such that $x, y \in \mathscr{N}$ and $\mathscr{N}$ is a minimal premouse. Since $\mathbb{R} \cap M \subseteq L[\vec{E}], \mathscr{N} \in L[\vec{E}]$, and $\mathscr{N}$ is a $\Pi_{2}^{1}$ mouse in $L[\vec{E}]$ by absoluteness. So $\mathscr{N}$ witnesses the right-hand side in $L[\vec{E}]$.

On the other hand, suppose $\mathscr{N}$ has in $L[\vec{E}]$ the properties on the right-hand side, and $x, y \in L[\vec{E}]$ and $y \leq_{c} x$. Working in $\mathscr{M}$ we can find a countable, minimal $\mathscr{P} \preccurlyeq\left(V_{\alpha}^{\mathscr{K}}, \in, \delta^{\mathscr{M}}, \mathscr{F}^{\mathscr{M}}, \bar{\nu}^{\mathscr{M}}\right)$ such that $x, y \in \mathscr{P}$. Then $\mathscr{P}$ is normally $\omega_{1}$-iterable in $\mathscr{M}$ and $\mathscr{N}$ is a $\Pi_{2}^{1}$ mouse in $\mathscr{M}$ by absoluteness. Since $\mathscr{N} \vDash x \leq_{c} y$ and $\mathscr{P} \vDash y \leq_{c} x$, no iterate of $\mathscr{N}$ is an initial segment of an iterate of $\mathscr{P}$, and vice-versa. This contradicts Lemma 6.13 in $\mathscr{M}$.

\section{ACKNOWLEDGMENTS}

The authors thank Robert Solovay and the referee for reading the paper carefully and making many helpful suggestions

\section{REFERENCES}

[B1] Stewart Baldwin, Generalizing the Mahlo hierarchy, with applications to the Mitchell models, Ann. Pure Appl. Logic 25 (1983), 103-127.

[B2] _ Between strong and superstrong, J. Symboic Logic 51 (1986), 547-559.

[D] Anthony Dodd, Strong cardinals, unpublished notes.

[DJ1] A. J. Dodd and R. B. Jensen, The core model, Ann. Math. Logic 20 (1981), 43-75.

[DJ2] _ The covering lemma for K, Ann. Math. Logic 22 (1982), 1-30.

[DJ3] _ The covering lemma for L[U], Ann. Math. Logic 22 (1982), 127-155.

[FMaSh] M. Foreman, M. Magidor, and S. Shelah, Martin's maximum, saturated ideals, and non-regular ultrafilters, Ann. of Math. (2) 127 (1988), 1-47.

[Ga] H. Gaifman, Elementary embeddings of models of set theory and certain subtheories of it, Axiomatic Set Theory (T. Jech, ed.), Proc. Sympos. Pure Math., vol. 13, Amer. Math. Soc., Providence, RI, 1969, pp. 103-126. 
[G] Kurt Gödel, Consistency-proof for the Generalized Continuum-Hypothesis, Proc. Nat. Acad. Sci. U.S.A. 25 (1939), 220-224.

[J1] R. B. Jensen, The fine structure of the constructible hierarchy, Ann. Math. Logic 4 (1972), 229-308.

[J2] Ronald B. Jensen, Reflections on a paper of Steel, unpublished notes.

[KMSo] Alexander S. Kechris, Donald A. Martin, and Robert M. Solovay, Introduction to Q-theory, Cabal Seminar 79-81, Lecture Notes in Math., vol. 1019, Springer-Verlag, New York, pp. 199-282.

[Ku] Kenneth Kunen, Some applications of iterated ultrapowers in set theory, Ann. Math. Logic 1 (1970), 179-227.

[MSo] Donald A. Martin and Robert M. Solovay, A basis theorem for $\Sigma_{3}^{1}$ sets of reals, Ann. of Math. (2) 89 (1969), 138-159.

[MS] Donald A. Martin and John R. Steel, A proof of projective determinacy, J. Amer. Math. Soc. 2 (1989), 71-125.

[Mi1] William J. Mitchell, Sets constructible from sequences of ultrafilters, J. Symbolic Logic 39 (1974), 57-66.

[Mi2] — Hypermeasurable cardinals, Logic Colloquium '78 (M. Boffa, D. Van Dalen, and K. McAloon, eds.), North-Holland, Amsterdam, 1979, pp. 303-317.

[Mi3] _ Sets constructible from sequences of measures: revisited, J. Symbolic Logic $\mathbf{4 8}$ (1983), 600-609.

[Mi4] _ The core model for sequences of measures. I, Math. Proc. Cambridge Philos. Soc. 95 (1984), 229-260.

[Mi5] _ The core model for sequences of measures. II, unpublished.

[MiS] William J. Mitchell and John R. Steel, Fine structure and iteration trees, to appear.

[ShW] Saharon Shelah and W. H. Woodin, Large cardinals imply that every reasonably definable set of reals is Lebesgue measurable, Israel J. Math. 70 (1990), 381-394.

[Si1] Jack H. Siler, The consistency of the GCH with the existence of a measurable cardinal, Axiomatic Set Theory (Dana Scott, ed.), Proc. Sympos. Pure Math., vol. 13, Amer. Math. Soc., Providence, RI, 1971, pp. 391-395.

[Si2] - Measurable cardinals and $\Delta_{3}^{1}$ well-orderings, Ann. of Math. (2) 94 (1971), 414-446.

[So1] R. M. Solovay, The cardinality of $\Sigma_{2}^{1}$ sets of reals, Found. Math. (J. Bulloff, T. Holyoke, and S. Hahn, eds.), Springer-Verlag, New York, 1969, pp. 58-73.

[So2] - The fine structure of $L[\mu]$, unpublished.

[St1] John R. Steel, Large cardinals and $\Delta_{3}^{1}$ wellorders, unpublished notes.

[St2] _ Inner models with many Woodin cardinals, to appear in Annals Pure Appl. Logic.

[W] W. H. Woodin, Supercompact cardinals, sets of reals, and weakly homogeneous trees, Proc. Nat. Acad. Sci. USA 85, 6587-6591.

Department of Mathematics, University of California at Los ANGeles, Los ANGeles, CALIFORNIA 90024-1555

E-mail address, D. A. MARTIN: DAM@MATH.UCLA.EDU

E-mail address, J. R. STEEL: STEEL@MATH.UCLA.EDU 